\title{
Time-dependent circumferential deformation of cortical bone subjected to internal radial loading
}

Christopher Uriah Brown

West Virginia University

Follow this and additional works at: https://researchrepository.wvu.edu/etd

\section{Recommended Citation}

Brown, Christopher Uriah, "Time-dependent circumferential deformation of cortical bone subjected to internal radial loading" (2001). Graduate Theses, Dissertations, and Problem Reports. 2336.

https://researchrepository.wvu.edu/etd/2336

This Dissertation is protected by copyright and/or related rights. It has been brought to you by the The Research Repository @ WVU with permission from the rights-holder(s). You are free to use this Dissertation in any way that is permitted by the copyright and related rights legislation that applies to your use. For other uses you must obtain permission from the rights-holder(s) directly, unless additional rights are indicated by a Creative Commons license in the record and/ or on the work itself. This Dissertation has been accepted for inclusion in WVU Graduate Theses, Dissertations, and Problem Reports collection by an authorized administrator of The Research Repository @ WVU.

For more information, please contact researchrepository@mail.wvu.edu. 


\title{
Time-Dependent Circumferential Deformation of Cortical Bone \\ Subjected to Internal Radial Loading
}

\author{
Christopher U. Brown
}

\author{
Dissertation submitted to the \\ College of Engineering and Mineral Resources \\ at West Virginia University \\ in partial fulfillment of the requirements \\ for the degree of
}

\author{
Doctor of Philosophy \\ in \\ Mechanical Engineering
}

Timothy L. Norman, Ph.D., Chair

Thomas A. Gruen, M.S.

Ever J. Barbero, Ph.D.

Victor H. Mucino, Ph.D.

J. David Blaha, M.D.

Department of Mechanical and Aerospace Engineering

\author{
Morgantown, West Virginia \\ 2001
}

Keywords: Bone Mechanics, Creep, Press-Fit, Total Hip Arthroplasty, Circumferential Strain, 


\section{ABSTRACT \\ Time-Dependent Circumferential Deformation of Cortical Bone Subjected to Internal Radial Loading}

\section{Christopher U. Brown}

Human cortical bone is a complex composite material that displays timedependent deformation. The response of bone to loads in the transverse orientation is not well understood and has implications to the stability of a press-fit hip implant. Total hip arthroplasty is a surgical procedure to replace the hip joint. It involves inserting an implant into the shaft of the femur. One method of implant fixation is a press-fit of the implant into the bone canal. Press-fit fixation relies on the elastic response of the proximal femur to hold the implant in place until new bone growth occurs. The radial load may cause the bone to experience creep, deformation due to a constant load, which can influence initial implant stability. The objectives of this study were to (a) study the time-dependent hoop response of femoral bone to an intramedullary radial load, (b) study the creep response of specimens under constant load until failure, and (c) assess damage morphology and determine the damage mechanisms in cortical bone due to a radial load.

A test fixture was used to apply internal pressure to cylindrical bone specimens. Hoop strain was measured on the surface of the cylindrical specimens. Creep strain, creep strain rate and permanent strain exhibited similar linear behavior at low stress, until a particular stress level, or threshold, where nonlinear behavior began. This deformation, which occurred at low hoop stress levels, may change the press-fit between the implant and bone, result in the loss of initial implant fixation, and may cause creep fracture. A relationship between hoop stress and time to failure was obtained and a hoop creep function was determined to model the creep strain behavior as a function of hoop stress and time. Fractured specimens were prepared for microscopic evaluation of damage morphology. Tensile hoop and compressive radial stresses resulted in extensive radial matrix microcracking combined with osteonal delaminations and may serve as a damage mechanism in cortical bone. The results from this study will provide fundamental knowledge regarding the creep behavior of bone in a similar loading environment to invivo press-fit loading. 


\section{ACKNOWLEDGEMENTS}

I wish to thank the many people who helped make this achievement possible. I would like to thank Dr. Tim Norman for the opportunity for conducting research in the bioengineering field and support during this project. I would also like to thank Mr. Tom Gruen for his guidance and insight into the clinical issues of this project. I would also like to thank my other committee members, Dr. J. David Blaha, Dr. Ever Barbero and Dr. Victor Mucino for their interest and constructive input.

I would like to thank Vincent Kish for his interest and patience during this project. His assistance, insight and time with the experimental design and testing were integral in the success of the creep testing. I wish to thank Nina Clovis and Suzanne Smith for their help with supplies and equipment. I wish to also thank Dr. Stanley Wearden for his statistical lessons and advice.

I would like to thank my family for their support over the years. I would like to especially thank my parents for their time and sacrifices to allow me the opportunity to obtain my education.

I would like to express my appreciation to my fellow graduate students. I wish to thank Keith Hustosky for his creative and constructive input and criticisms. I wish to thank Gagik Parsamian for his help in appreciating the theoretical aspects of this project and for his assistance in the analysis of the damage morphology. I would also like to thank Ganesh Thyagarajan for his programming and computer expertise. Finally, I would like to thank Anna Christina Salcedo for her support and her infinite supply of patience over the years. 


\section{TABLE OF CONTENTS}

ABSTRACT II

ACKNOWLEDGEMENTS III

TABLE OF CONTENTS IV

LIST OF FIGURES VII

LIST OF TABLES XIII

NOMENCLATURE XIV

CHAPTER 1 INTRODUCTION 1

1.1 Project Overview 1

1.2 Objectives 1

1.3 Hypotheses 2

1.4 Anatomical Background 2

1.4.1 Macrostructure of the Femur 3

1.4.2 Microstructure of the Femur 4

1.5 Clinical Significance $\quad 7$

1.5.1 Clinical Hip Fracture 9

1.5.2 Intraoperative Fractures 10

1.5.3 Effect of Creep on Hip Arthroplasty and Stability 11

$\begin{array}{ll}\text { 1.5.4 Long Femoral Implants } & 13\end{array}$

1.5.5 Uncemented Hip Implant List 13

CHAPTER 2 VISCOELASTICITY AND CREEP BEHAVIOR 19

2.1 Definitions $\quad 19$

2.2 Thick Wall Cylinders 24

2.3 Previous Investigations of Time-Dependent Bone Deformation 26

2.3.1 Viscoelastic Behavior of Longitudinally Oriented Bone Specimens 26

2.3.2 Time-Dependent Behavior of Transversely Oriented Bone Specimens $\quad 38$

2.4 Mechanisms of Creep Deformation 39

CHAPTER 3 SPECIMEN MACHINING AND TESTING EQUIPMENT 53

3.1 Specimen Selection and Bone Availability 53

3.2 Specimen Preparation 53

3.3 Inner Cortex Reaming 55

3.4 Outer Cortex Reaming $\quad 57$

3.5 Electrical Resistance Strain Gage Application $\quad 58$

$\begin{array}{ll}\text { 3.6 Measuring Strain from the Gages } & 60\end{array}$ 
3.7 The Testing Fixture $\quad 60$

$\begin{array}{ll}3.8 \text { Experiment equipment } & 63\end{array}$

3.9 Strain Magnitudes on Whole Femurs 65

CHAPTER 4 PRELIMINARY WORK 67

4.1 Experimental Device for Applying Internal Pressure to Cylindrical Specimens (Brown et al., 2000a, Experimental Techniques) 67

4.1.1 Introduction $\quad 67$

$\begin{array}{ll}\text { 4.1.2 The Cylindrical Specimens } & 67\end{array}$

4.1.3 Test Procedure $\quad 68$

4.1.4 Data Analysis for the Aluminum Specimen $\quad 70$

4.1.5 Data Analysis for the Femoral Bone Specimens 70

4.1.6 Results and Discussion $\quad 71$

4.2 Circumferential Strain Around Femoral Cylinders Due to Simulated Press-Fit Loading (Brown et al., 1998)

4.2.1 Introduction 74

4.2.2 Materials and Methods $\quad 75$

4.2.3 Results $\quad 76$

4.2.4 Discussion 77

4.3 Transverse Creep Response in Human Femoral Bone (Brown et al., 1999) 77

4.3.1 Introduction 77

4.3.2 Materials and Methods 78

$\begin{array}{ll}4.3 .3 \text { Results } & 78\end{array}$

4.3.4 Discussion $\quad 80$

CHAPTER 5 SHORT-TERM CREEP TESTS 82

$\begin{array}{ll}5.1 \text { Introduction } & 82\end{array}$

5.2 Materials and Methods $\quad 82$

5.2.1 Setting the Pressure $\quad 83$

5.2.2 Multi-Phase Testing Protocol 85

5.2.3 Morphological and Statistical Analysis $\quad 87$

5.3 Hoop Stiffness (Instantaneous Modulus) 89

5.4 Strain Rate During Pressurization 94

5.5 Static Modulus 96

5.6 Creep Strain 97

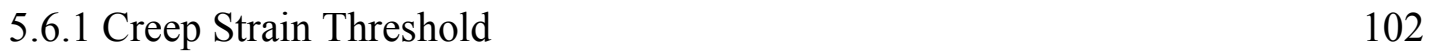

5.6.2 Multivariable Model of Creep Strain 105

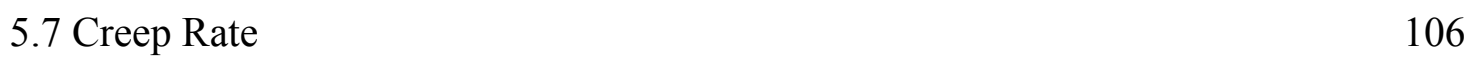

5.7.1 Creep Rate Threshold 108

5.7.2 Multivariable Model of Creep Rate $\quad 110$

5.8 Permanent Strain $\quad 111$

5.8.1 Permanent Strain Threshold 113 
5.8.2 Multivariable Model of Permanent Strain 115

5.9 Material Assumption for Bone 116

$\begin{array}{ll}5.10 \text { Discussion } & 117\end{array}$

CHAPTER 6 LONG-TERM CREEP TESTS 122

$\begin{array}{ll}6.1 \text { Introduction } & 122\end{array}$

6.2 Materials and Methods 122

6.2.1 Testing Protocol 124

6.2.2 Morphological and Statistical Analysis 125

6.3 Time to Failure Results 126

6.4 Creep Model Results 133

6.4.1 The General Creep Model 135

6.4.2 The Short-Term Creep Model 136

6.4.3 The Long-Term Creep Model 139

$\begin{array}{ll}6.5 \text { Discussion } & 146\end{array}$

$\begin{array}{ll}\text { 6.5.1 Time to Failure } & 148\end{array}$

6.5.2 The Creep Model 149

CHAPTER 7 FAILURE MECHANISMS IN RADIAL LOADED BONE 151

7.1 Introduction $\quad 151$

7.2 Materials and Methods 151

$\begin{array}{ll}7.3 \text { Results } & 155\end{array}$

$\begin{array}{ll}7.4 \text { Discussion } & 159\end{array}$

CHAPTER 8 SUMMARY, CONTRIBUTIONS, RECOMMENDATIONS 163

8.1 Summary 163

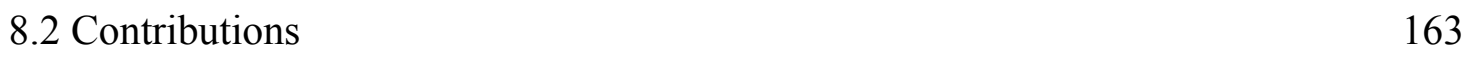

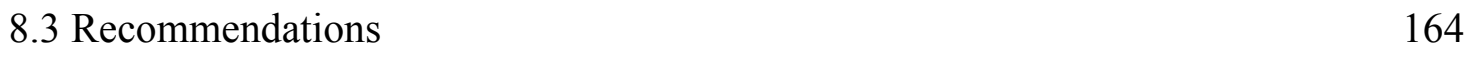

REFERENCES

$\begin{array}{lr}\text { CURRICULUM VITAE } & 184\end{array}$ 


\section{LIST OF FIGURES}

Figure 1.1 Anatomical positions on the human femur. 2

Figure 1.2 Longitudinal, radial and circumferential or hoop directions for bone. The radial and hoop directions are in the transverse plane. 3

Figure 1.3 Anatomical aspects of the human femur. 4

Figure 1.4 The microstructure of the femur includes hydroxyapatite crystals attached to the collagen fibers. The wound fibers form layers of the osteon called lamella (Park, 1979).

Figure 1.5 Structure of the osteons of cortical bone (Junqueira and Carneiro, 1992). 6

Figure 1.6 Cross-sections of human cortical bone stained with hematoxylin and eosin. (A) Haversian canals are dark stained regions within osteons. (B) The osteons have a cement line surrounding them that separates them from the bone matrix. (C) Microcracks in the bone matrix can be seen on the middle right.

Figure 1.7 Radial forces from the press-fit implant result in tensile hoop strain in the bone.

Figure 2.1 A stress strain curve displaying linear and non-linear behavior. 19

Figure 2.2 (a) The elastic response where deformation is recovered and (b) the plastic response where deformation is not completely recovered. 20

Figure 2.3 (a) Viscoelastic material response where deformation is recovered with time and (b) a viscoplastic material response where deformation is not recovered in time.

Figure 2.4 (a) At a constant strain a viscoelastic material may require less stress to maintain the deformation over time or relax. (b) A viscoelastic material may creep, or deform under constant load over time.

Figure 2.5 (A) The three stages of creep and (B) strain rate. During primary creep, the strain rate decreases after an initial load is applied. The minimum strain rate is maintained during secondary creep. Finally, the strain rate increases until failure. The shape of all three stages will depend on load rate, load level and load orientation (tension vs. compression).

Figure 2.6 For a viscous material, the instantaneous elastic strain, $\varepsilon_{0}$, is followed by continued deformation during the constant load. Once the load is removed, an initial elastic recovery is followed by gradual recovery. Some permanent strain may remain depending on the load and the material.

Figure 2.7 The transverse plane is perpendicular to the longitudinal plane. 38

Figure 2.8 The hierarchal structure of bone including osteons, lamella, collagen fibers and collagen molecules (Lakes, 1993).

41

Figure 2.9 The three helices of a tropocollagen molecule wrap tightly around one another. The figure shows one end of the molecule, looking down the center axis with glycine residues stacked along the center (Lehninger et al., 1993, p. 172). 42

Figure 2.10 The tropocollagen is made of three helices. The collagen fibril has tropocollagen staggered five rows across. An electron micrograph shows the striated appearance due to the gap regions (Garrett and Grisham, 1999, p. 177; Lehninger et al., 1993, p. 174).

Figure 2.11 Intra-tropocollagen cross-links: 1. Cross-links between lysine residues at the nonhelical N-terminal region; 2. Glycine hydrogen bonds to other amino acid 
residues; 3 . Hydroxyproline hydrogen bonds between strands.

Figure 2.12 Inter-tropocollagen cross-links: Hydroxypyridinium structure composed of two hydroxylysines at the $\mathrm{C}$-terminal and one lysine amino acid residue at the $\mathrm{N}$ terminal.

Figure 2.13 The three modes of deformation within a fibril as defined by Sasaki and Odajima (1996). Mode 1 is tropocollagen elongation, mode 2 is elongation of the gap region, and mode 3 is slippage between laterally adjoining tropocollagen molecules.

Figure 2.14 The hydrogen bonds between the three stands of a tropocollagen molecule, thin cross-section view. The hydrogen bonds are represented by dots and $\mathrm{G}$ is the alpha carbon of the glycine amino acid residue. (Stryer, 1995, p. 32).

Figure 2.15 Fibers oriented in the hoop direction may deform as suggested for tendons, however, fibers oriented in the longitudinal direction may not deform. $\quad 50$

Figure 2.16 Deformation mechanisms of collagen fibril under hoop stress. 51

Figure 3.1 AP and ML views of the right femur. The two $\mathrm{x}$-rays were taken perpendicular to one another.

Figure 3.2 U-clamp device for holding the femoral shaft for making the transverse cut. 54

Figure 3.3 A bulk section, $19 \mathrm{~mm}$ in length, was removed from the femoral shaft approximately $95 \mathrm{~mm}$ distal to the lesser trochanter.

Figure 3.4 Milling plan for removal of inner cortex of the bone. 56

Figure 3.5 Bone reaming fixture for removal of the inner cortex of the bone. 56

Figure 3.6 The expanding mandrel. $\quad 57$

Figure 3.7 The milling path for the outer cortex of the bone. 58

Figure 3.8 The cross section of bone with strain gages at each quadrant and along the mid-line of the specimen.

Figure 3.9 The test fixture with the cylindrical specimen (1), two aluminum end caps (2), two aluminum hose plugs (3), a $60 \mathrm{~mm}$ length latex rubber hose (4), four tie rods to hold the device together (5), two end plate clamps to restrict the horizontal travel of the hose plugs (6), a stopper plug to prevent gas from leaking out of the hose (7), and four spacer washers (8); cross-sectional view.

Figure 3.10 The test fixture with the bone specimen. 62

Figure 3.11 The experiment set-up. $\quad 63$

Figure 3.12 Pressure and microstrain versus time for an aluminum specimen (file=adjRate6b).

Figure 4.1 The aluminum cylinder with one longitudinal and one hoop strain gage. 68

Figure 4.2. The bone cylinder with hoop strain gages along the mid-line of the specimen.

Figure 4.3 Measured hoop and longitudinal strain and internal pressure versus time for the aluminum specimen. A linear relationship between the measured strain and the internal pressure is evident.

Figure 4.4 The relationships between measured $\left(\mathrm{R}^{2}=0.99\right)$ and calculated hoop strain versus internal pressure are similar for the aluminum specimen and suggests the testing device is accurate. Regression equation: Measured $\varepsilon_{\mathrm{H}}=83.76 \mathrm{P}_{\mathrm{i}}-8.98 \quad 73$

Figure 4.5 Hoop stiffness over the pressure range for the first (A) and second (B) aluminum test using a thinner aluminum specimen. The variation between the two gages is most likely due to the uneven specimen thickness. 
Figure 4.6 The bone cylinder, with a thickness of $2.4 \mathrm{~mm}$ and gages to measure hoop strain.

Figure 4.7 The mean strain was determined from the 100 second span. 75

Figure 4.8 Mean hoop strain from each cortical region around the circumference of the four femoral cylinders.

Figure 4.9 Specimen \#1, failure occurred between 31800 and 31900 seconds at a pressure of $2.07 \mathrm{MPa}(300 \mathrm{psi})$.

Figure 4.10 Specimen \#3, failure occurred between 3700 and 3800 seconds at a pressure of 2.01 MPa (292 psi).

Figure 4.11 Specimen \#4, failure occurred between 64 and 64.5 seconds at a pressure of $1.68 \mathrm{MPa}(243 \mathrm{psi})$.

Figure 4.12 Transverse elastic and creep strain of cortical bone, specimen \#3 anterior gage, subjected to an internal pressure of $2 \mathrm{MPa}(292 \mathrm{psi})$. A cubic spline was used to fit the data.

Figure 5.1 The testing equipment for the short-term creep tests.

Figure 5.2 A pressure of $0.345 \mathrm{MPa}(50 \mathrm{psi})$ was applied initially for the first phase of one minute of pressure and increased during each cycle. These are the pressure levels for one specimen where the final pressure level resulted in failure (Test 8). 85

Figure 5.3 The four phases of the loading cycle: phase 1, pressurization; phase 2, constant pressure; phase 3, unloading; phase 4 , zero pressure (Test 8 , final cycle). 86

Figure 5.4 The measured parameters from the short-term tests: creep strain, creep rate and permanent strain.

Figure 5.5 A typical hoop stress-strain curve for the loading portion of a sample stress level (Test 8d). The hoop stiffness is $7.33 \mathrm{GPa}(1.06 \mathrm{E} 6 \mathrm{psi})$.

Figure 5.6 The initial hoop stiffness for each quadrant (pressure $=0.345 \mathrm{MPa}(50 \mathrm{psi})$ ) for all specimens $(\mathrm{n}=15)$. Significant differences were found between the medial and posterior quadrants $(\mathrm{p}=0.04)$ and between the medial and lateral quadrants $(\mathrm{p}=0.038)$. The mean initial hoop stiffness is included with the standard error in parenthesis.

Figure 5.7 Hoop stiffness decreased due to damage during repeated load cycles. $\quad 92$

Figure 5.8 The hoop stiffness for all specimens at each quadrant and a power fit: (A) posterior, (B) medial, (C) anterior and (D) lateral quadrants. $\quad 93$

Figure 5.9 Power fits of the hoop stiffness for all four quadrants throughout the hoop stress range.

Figure 5.10 The strain rate, $958.63 \mu \varepsilon / \mathrm{s}$, was determined as the slope of the linear portion, between 200.45 and 200.65 seconds, where the load was applied at a constant rate, $1.64 \mathrm{MPa} / \mathrm{s}(238.63 \mathrm{psi} / \mathrm{s}$ ) (region bounded by the dotted lines) (Test $8 d)$.

Figure 5.11 (A) A hoop stress-strain curve for a single specimen showing the maximum hoop strain during at each hoop stress level. (B) The static modulus is determined from the slope of the curve of the initial linear region, $\sigma_{\mathrm{H}}<4.71 \mathrm{MPa}(684.09 \mathrm{psi})$, of the hoop stress versus maximum hoop strain (posterior, Test 8). The static modulus is $5.69 \mathrm{GPa}(0.83 \mathrm{E} 6 \mathrm{psi})$.

Figure 5.12 (A) Creep strain measured from the hoop strain during the 1-minute of constant pressure (Test $8 \mathrm{i}$ ). The creep strain, $853 \mu \varepsilon$, was calculated as the difference between the initial strain, $1849.3 \mu \varepsilon$, and the final strain after 1 minute, $2702.3 \mu \varepsilon$. 
(B) Creep strain increases with increasing hoop stress. 98

Figure 5.13 Creep strain versus hoop stress for all 15 specimens from all quadrants. 99

Figure 5.14 Creep strain variation with hoop stress normalized with hoop stiffness from each stress level. The exponential fit provided the best curve. 100

Figure 5.15 Creep strain variation with normalized hoop stress for each quadrant, posterior (A), medial (B), anterior (C) and lateral (D). An exponential curve was used to fit the data in each plot.

101

Figure 5.16 Creep strain measured during the normalized hoop stress levels that resulted in linear behavior $\left(\sigma_{\mathrm{H}} / \mathrm{E}_{\mathrm{H}}<0.0005\right)$. The dashed line was offset from the linear fit of the data represented by the solid line.

Figure 5.17 The threshold is the intersection between the exponential fit of the data and the offset line.

104

Figure 5.18 (A) The creep rate $(7.5 \mu \varepsilon / \mathrm{s})$ during the last 40 seconds of the 1 -minute load phase (pressure $=2.08 \mathrm{MPa}(301.68$ psi)) (Test 8i). (B) As the hoop stress levels increase, the creep rate also increases.

106

Figure 5.19 Creep rate for all 15 specimens from all quadrants for all hoop stress levels normalized with hoop stiffness, $\mathrm{p}<0.0001$.

107

Figure 5.20 Creep rate measured during the normalized hoop stress levels that resulted in linear behavior $\left(\sigma_{\mathrm{H}} / \mathrm{E}_{\mathrm{H}}<0.0005\right)$. The dashed line was offset from the linear fit of the data represented by the solid line.

108

Figure 5.21 The creep rate threshold was the intersection between the exponential fit of all the data and the offset line.

109

Figure 5.22 (A) Permanent strain, $300.04 \mu \varepsilon$, measured as the strain after 2 minutes of zero pressure (Test 8i). (B) Permanent strain increases with increasing levels of hoop stress.

111

Figure 5.23 Permanent strain variation with hoop stress normalized with hoop stiffness from each stress level, $\mathrm{p}<0.0001$.

Figure 5.24 Plastic strain measured during the normalized hoop stress levels that resulted in linear behavior $\left(\sigma_{H} / E_{H}<0.0005\right)$. The dashed line was offset from the linear fit of the data represented by the solid line.

113

Figure 5.25 The plastic strain threshold was the intersection between the fourth order polynomial fit and the offset line.

114

Figure 5.26 Hoop stress distribution based on elastic and creep deformation of a thick $\begin{array}{ll}\text { walled cylinder. } & 117\end{array}$

Figure 6.1 The experiment set-up for the long-term tests.

123

Figure 6.2 The three stages of creep strain for a specimen (Test 25) with internal pressure of 2.21 MPa (321 psi). Creep failure occurred after 2.21E+04 seconds (6.14 hours).

Figure 6.3 Pressure versus time to failure for all 15 specimens. There were seven general pressure levels: $1.86 \mathrm{MPa}$ (270 psi), 2.07 MPa (300 psi), 2.20 MPa (320 psi), 2.34 $\mathrm{MPa}$ (340 psi), 2.41 MPa (350 psi), 2.48 MPa (360 psi) and 2.76 MPa (400 psi). Note one specimen (Test 23) did not fail after 14 days of constant pressure. 128

Figure 6.4 Normalized hoop stress and time to failure for all specimens. (A) A logarithmic fit was the best curve for the data. (B) A log-log plot of normalized hoop stress and time to failure allows the data at early times of failure to be observed. The fits of the data in Figure 6.4 were included, despite the non-significant relationship, 
for comparison to Figure 6.5.

Figure 6.5 A significant relationship between normalized hoop stress and time to failure excluding Test \# 20 and 21, p=0.026, on a linear plot (A) and a logarithmic plot (B).

Figure 6.6 Caler and Carter's (1989) power fit for longitudinal creep fracture for the normalized stress level in this study compared to the power fit for hoop creep fracture for hoop specimens.

Figure 6.7 Hoop creep strain from each quadrant for all 15 specimens. The numbers in the plots represent the test number (19-33). The end of the strain-traces was either where the strain entered the third stage of creep or where the strain gage failed due to saline migration. The range of time on the $\mathrm{x}$-axis was chosen to allow the strain at early times to be observed. The specimen strain labeled with an arrow ends at the tip of the arrow.

Figure 6.8 Measured creep strain and the power and exponential creep model for the different stress levels for the initial 6 hours $(2.16 \mathrm{E}+04$ seconds). A representative constant hoop stress was applied to the creep models which is close to the hoop stress level applied to the specimens. It is also important to note that the creep model was derived from measured strain from 15 specimens; it is not a curve fit of the strain data within a single plot.

138

Figure 6.9 Measured creep strain and power and exponential creep models for the different stress levels for the duration of each test. A representative constant hoop stress was applied to the creep models which was close to the hoop stress level applied to the specimens. It is also important to note that the creep model was derived from measured strain from 10 specimens; it is not a curve fit of the strain data within a single plot.

Figure 6.10 The power creep model for the behavior during the initial 6 hours for the maximum, minimum and medium stress levels. A difference in creep behavior was evident, especially at higher levels of hoop stress.

142

Figure 6.11 (A) The power and the exponential fits for the initial 6 hours and (B) for several days. Both models were very similar at all hoop stress levels.

Figure 7.1 Four fields, $651 \mu \mathrm{m}$ by $651 \mu \mathrm{m}$, were used to measure microcracks at each quadrant.

152

Figure 7.2 Different types of microdamage within and around an osteon in human cortical bone.

Figure 7.3 (A) Microcracks at the endosteal surface and (B) at the periosteal surface. A greater number of cracks were observed at the endosteal surface. Images are $651 \mathrm{x}$ $651 \mu \mathrm{m}^{2}$.

Figure 7.4 Images at the endosteal surface of the (A) untested, control, specimen and (B) a tested specimen. Microdamage density and microdamage surface density were greater for the tested specimens than for the control.

156

Figure 7.5 (A) Radial crack density was significantly greater than hoop crack density for the tested specimens $(\mathrm{p}<0.05)$ and $(\mathrm{B})$ crack density versus anatomical region for the five tested specimens, where the anterior quadrant has a significantly greater radial crack density than hoop crack density $(\mathrm{p}<0.05)$.

Figure 7.6 More radial cracks were found compared to hoop oriented cracks. (A) Microcracks are extending between cement lines of two osteons and into the matrix 
of cortical bone. Cracks along the cement line are visible around the bottom left osteon ( $325.5 \times 325.5 \mu \mathrm{m}^{2}$ ); (B) magnified image taken from within the white box of (A), $\mathrm{H}$ indicates a microcrack in the hoop direction and $\mathrm{R}$ indicates a crack in the radial direction $\left(162.75 \times 162.75 \mu^{2}\right)$.

Figure 7.7 The percentages of the different types of microdamage for the five tested specimens.

158

Figure 7.8 Microdamage in the matrix or in the matrix and along a cement line was the most frequent form of damage.

Figure 7.9 The internal pressure results in a combination of large tensile hoop stress and a smaller radial stress at the inner surface. Only hoop stress exists at the outer surface.

Figure 7.10 Tensile hoop and compressive radial stress tend to open cracks in the radial direction and cause delaminations between the cement lines and bone matrix. $\quad 160$ 


\section{LIST OF TABLES}

Table 1.1 List of uncemented stems and recommended size difference between stem and femoral canal.

Table 2.1 Bone creep investigations with respect to type of loading and specimen geometry. Long. = Longitudinally oriented specimen, Trans. = Transverse, tangentially oriented specimen, rect. $=$ rectangular geometry, cyl $=$ cylindrical geometry

Table 4.1 Internal pressure, measured and calculated hoop and longitudinal strain and the ratio of longitudinal to hoop strain for the aluminum specimen.

Table 4.2 Mean measured and calculated hoop strain for the femoral bone specimens at a pressure of $0.69 \mathrm{MPa}$.

Table 4.3 Mean hoop strain and standard error of each cortical region. 76

Table 4.4 Constant internal pressure magnitudes and time-to-failure for the bone specimens.

Table 5.1 Distribution of specimens with respect to gender and age. Note that specimen tests 1,2 and 3 were practice specimens.

Table 5.2 The mean initial hoop stiffness values from each quadrant determined from the initial pressurization of $0.345 \mathrm{MPa}(50 \mathrm{psi})$ for each specimen $(\mathrm{n}=15$ for each quadrant).

Table 5.3 Strain rates and corresponding stiffness values measured in different studies while loading specimens in tension.

Table 5.4 The mean hoop stiffness values from each quadrant determined from the entire hoop stress range.

Table 5.5 The static modulus for each of the 15 specimens at each quadrant.

Table 5.6 Equations of various fits for hoop creep strain versus normalized hoop stress.

Table 5.7 Multivariable model of creep strain.

Table 5.8 Equations of various fits for hoop creep rate versus normalized hoop stress. 108

Table 5.9 Multivariable model of creep rate.

Table 5.10 Equations of various fits for permanent strain versus normalized hoop stress.

Table 5.11 Multivariable model of permanent strain.

Table 6.1 Distribution of specimens with respect to gender and age.

123

Table 6.2 The constant internal pressure values for each specimen that were maintained until specimen failure, constant hoop stress values calculated using the internal pressure, and time to failure. Test \# 23 did not fail after 14 days.

Table 6.3 Equations of various fits for normalized hoop stress versus time to failure. 129

Table 6.4 Equations of various fits for normalized hoop stress versus time to failure excluding Test \# 20 and 21.

Table 6.5 Multivariable model of time to failure.

Table 6.6 Multivariable model of long-term creep strain.

Table 7.1 Microdamage type including description. 


\section{NOMENCLATURE}

A: Anterior

DAS: Data acquisition system

$\mathrm{d} \varepsilon / \mathrm{dt}$ : Strain rate

$\mathrm{d} \sigma / \mathrm{dt}$ : Stress rate

$\Delta \varepsilon$ : Change in strain

E: Elastic modulus

$\mathrm{E}_{\mathrm{H}}$ : Elastic modulus in the hoop direction

$\mathrm{E}_{\mathrm{L}}$ : Elastic modulus in the longitudinal direction

$\varepsilon:$ Strain

$\varepsilon_{\mathrm{H}}$ : Strain in the hoop direction

$\varepsilon_{\mathrm{L}}$ : Strain in the longitudinal direction

$\varepsilon_{\mathrm{r}}$ : Strain in the radial direction

$\varepsilon_{\theta}$ : Strain in the hoop direction

$\varepsilon_{\mathrm{z}}$ : Strain in the longitudinal direction

GPa: Gigapascal

In.St: Initial stiffness

L: Lateral

M: Medial

mm: Millimeter

MPa: Megapascal

$\mathrm{mV}$ : Millivolts

$\mu \varepsilon$ : Microstrain

$\mu \mathrm{m}$ : Micrometer

v: Poisson's ratio

On.Ar: Osteon area

On.Dn: Osteon density

P: Posterior

Pa: Pascal 
$\mathrm{p}_{\mathrm{i}}$ : Inner pressure

Po: Porosity

$\mathrm{p}_{\mathrm{o}}$ : Outer pressure

psi: Pressure per square inch

$\mathrm{R}$ : Correlation coefficient

r: Radius

$r^{2}$ : Coefficient of determination

$\mathrm{r}_{\mathrm{i}}$ : Inner radius

$\mathrm{r}_{\mathrm{o}}$ : Outer radius

S: Compliance matrix

s: Second

s.On.Ar: Single osteon area

St.D.: Standard deviation

$\sigma_{\mathrm{r}}$ : Stress in the radial direction

$\sigma_{\theta}:$ Stress in the hoop direction

$\sigma_{\mathrm{u}}$ : Ultimate stress

$\sigma_{\text {ult: }}$ Ultimate stress

$\sigma_{\mathrm{z}}:$ Stress in the longitudinal direction

t: Time

$\mathrm{t}_{\mathrm{f}}$ : Time to failure

Tt.Ar: Total area

Tt.N.On: Total osteon number

Tt.On.Ar: Total osteon area

Tt.Po.Ar: Total pore area

${ }^{\circ} \mathrm{C}$ : Degree centigrade

\#: Number 


\section{CHAPTER 1 \\ INTRODUCTION}

\subsection{Project Overview}

Human cortical bone is a complex composite material with elastic and failure properties that differ with direction. Knowledge of these properties is important for the development of accurate material behavior models used in analyses of bone and in design of joint prostheses. Bone has also demonstrated time-dependent behavior in laboratory tests of longitudinally oriented specimens. Creep, increasing deformation under constant load, has been observed. The response of bone to loads in the transverse, or circumferential, direction is less understood even though initial stability of a "press-fit" intramedullary joint prosthesis depends largely on circumferential support. A test fixture was used to apply internal pressure to cylindrical bone specimens in an idealized loading environment similar to a press-fit implant in bone. Circumferential, or hoop, strain was measured from the outer surface of the specimens. A multi-phased loading protocol was used to observe creep behavior in the specimens subjected to increasing pressure loads until failure. A second protocol was also used to observe creep behavior at a single pressure level until the specimens failed. A range of pressure levels was applied to obtain a relationship between creep strain, constant stress and time. Confocal microscopy was used to examine microdamage. The results from this study provide fundamental knowledge of the creep behavior of bone in an idealized loading environment similar to in-vivo press-fit loading.

\subsection{Objectives}

To better understand the response of bone in the hoop direction several objectives were established:

(1) to determine hoop creep thresholds of femoral bone subjected to an intramedullary radial load to understand how bone deforms due to stresses that may occur as a result of a press-fit hip prosthesis. Specifically, a hoop creep strain, creep strain rate and permanent strain threshold will be determined. The thresholds are hoop stress values after which significant hoop creep strain occurs and increases nonlinearly. 
(2) to determine the creep response of bone specimens under constant load until failure to establish a creep model and a relationship between time to failure and stress.

(3) to assess damage morphology to determine the damage mechanisms in cortical bone due to a radial load to provide insight into how damage occurs in human cortical bone.

\subsection{Hypotheses}

The hypotheses of this research are:

(1) hoop creep strain in bone increases nonlinearly with increasing hoop stress above a threshold value.

(2) radially loaded cortical bone exhibits creep behavior and may be modeled using creep strain as a function of hoop stress and time.

(3) damage as a result of a radial load in bone occurs at the inner cortex and is primarily oriented in the radial direction.

\subsection{Anatomical Background}

Terms are used to define anatomical positions of the human body as well as the body components. The subject of this investigation is the human femur, the thighbone. Anterior and posterior are terms indicating the front and back, respectively (Figure 1.1). Medial is used to describe a position facing the middle of the body while lateral refers to the position facing away from the middle. Proximal is a term used to describe a position close to the trunk, or the hip joint in this case, while distal is away from the trunk.

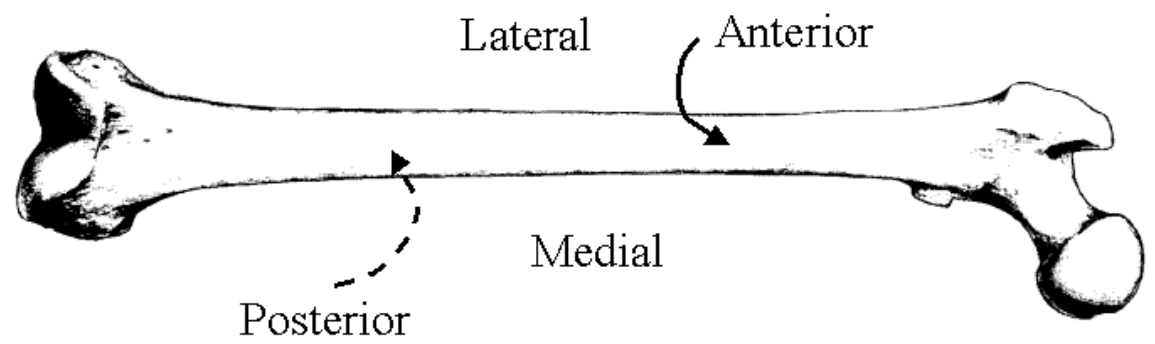

Distal

Proximal

Figure 1.1 Anatomical positions on the human femur. 
For the femur, the longitudinal direction is along the length of the bone while the transverse plane is perpendicular (Figure 1.2). The radial direction passes through the center of the bone's longitudinal axis, following the radius of the bone's cylindrical shape. The circumferential or hoop direction passes around the longitudinal axis.
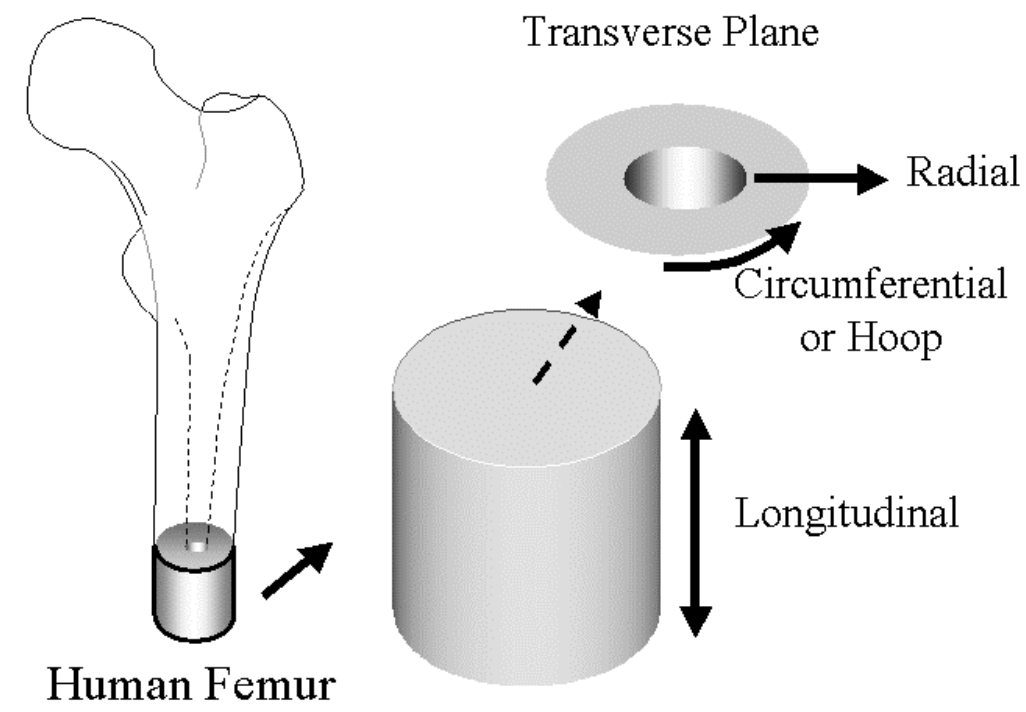

Figure 1.2 Longitudinal, radial and circumferential or hoop directions for bone. The radial and hoop directions are in the transverse plane.

\subsubsection{Macrostructure of the Femur}

The human femur is a long bone of the skeleton. It articulates at the proximal end with the coxal bone of the pelvis forming the hip joint. At the distal end, the femur articulates with the tibia and the patella to form the knee joint. The proximal end of the femur consists of the head, neck and lesser and greater trochanter (Figure 1.3). In general, the femur has two types of bone structure, cortical and trabecular. Cortical bone tends to be very dense and is thickest along the shaft or diaphysis. It is covered with a double layer of dense connective tissue called the periosteum. Trabecular bone is less dense and is found at the distal and proximal ends on the interior of the femur. The short-segmented pieces of trabeculae give the material a spongy appearance and a large surface area. A smooth transition exists between cortical bone on the exterior and the trabecular bone on the interior. 


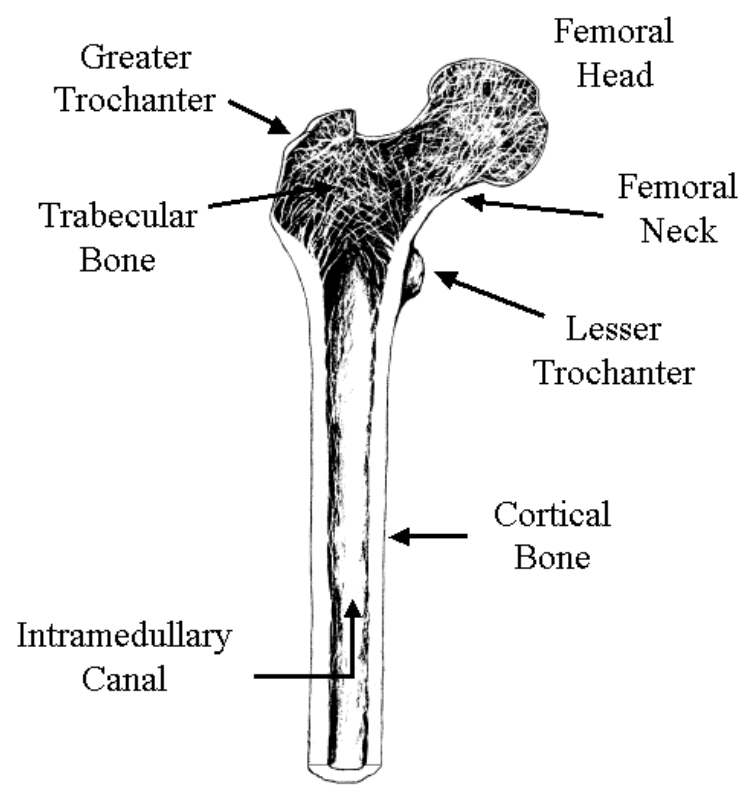

Figure 1.3 Anatomical aspects of the human femur.

\subsubsection{Microstructure of the Femur}

The microstructure of bone has two components: cells and an extracellular matrix. The cellular component consists of bone forming cells, osteoblasts that follow osteoclasts. Osteoclasts are the bone destroying cells that absorb microcracks. The extracellular matrix is comprised of an organic phase and an inorganic phase. The organic phase consists of osteoid that contains collagen, micro-proteins and lipids (Figure 1.4). The collagen content is $90-95 \%$ of the organic matrix and is responsible for preventing tensile stress in bone. The tensile stress at fracture and the fracture mode have been found to be highly dependent on the direction and orientation of the collagen fibers (Simkin and Robin, 1974).

The inorganic phase of the extracellular structure is made of calcium crystals. These crystals are mostly in the form of hydroxyapatite. This mineral composition provides excellent compressive strength for bone. The mineral content has a direct effect on the mechanical properties of bone. Currey (1988) determined a strong positive relationship between mineral content and elastic modulus, and Jurist and Foltz (1977) found a high correlation between mineral content and strength. Mineral content was not found to change significantly with age in mature bone (McCalden et al., 1993). 


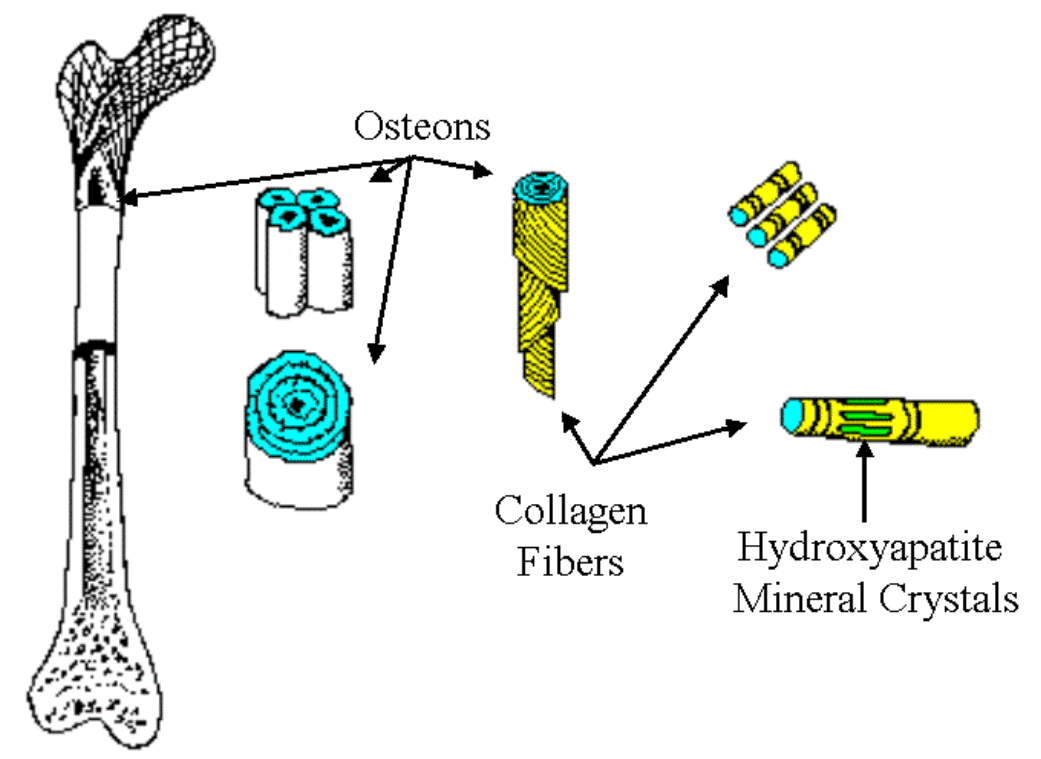

Figure 1.4 The microstructure of the femur includes hydroxyapatite crystals attached to the collagen fibers. The wound fibers form layers of the osteon called lamella (Park, 1979).

Human cortical bone is a living tissue with a complex hierarchal structure. Bone has a microstructural organization similar to fiber-reinforced composites with transversely isotropic or orthotropic material symmetry (Figure 1.5). The osteons, surrounded by a cement line, are similar to fibers within an interstitial matrix. Vascular channels, such as Volkmann's and Haversian canals and pores in bone are similar to voids in composite materials.

Secondary bone is a product of bone resorption, the replacement of previously existing bone tissue. The new bone has a central vascular channel called the Haversian canal (Figure 1.5). The Haversian canal supplies nutrients to the bone. The canal is surrounded by a series of concentric lamellae containing osteocytes. The entire system is surrounded by a cement line that separates the osteon from the interstitial matrix. The osteons tend to be oriented in the direction that the bone is loaded, in the case of the middiaphysis, the osteons are oriented in the longitudinal direction. 


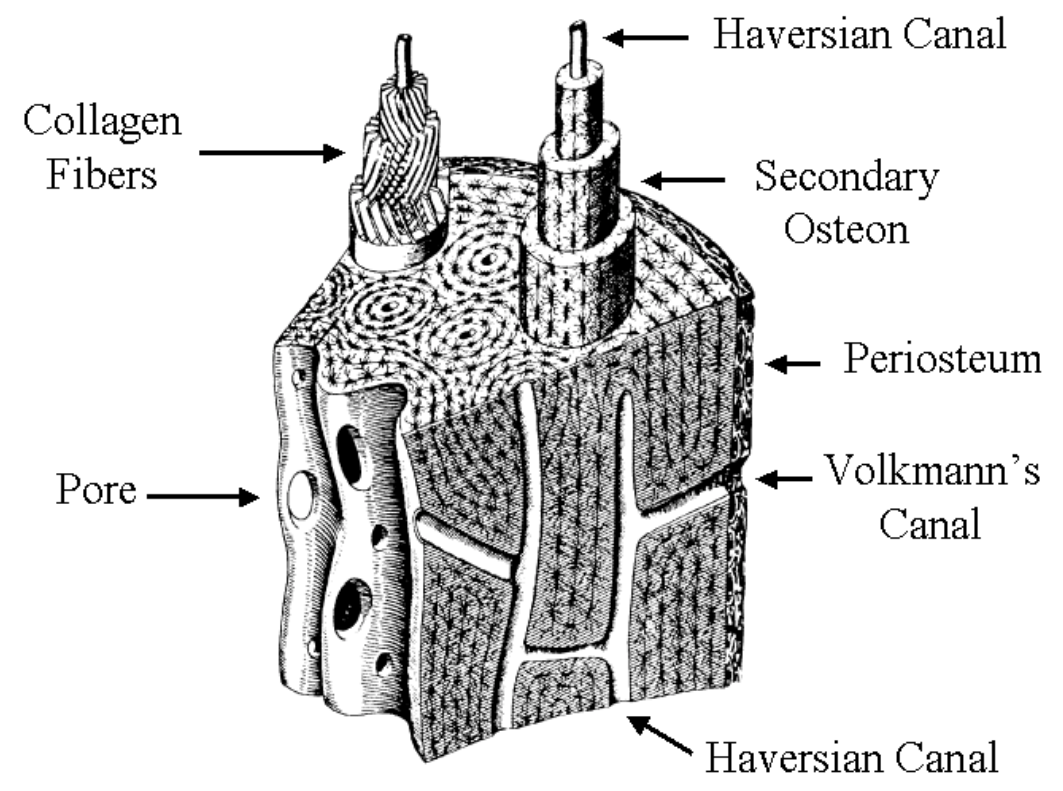

Figure 1.5 Structure of the osteons of cortical bone (Junqueira and Carneiro, 1992).

Osteons play an important dual role in the mechanical properties of bone (Figure 1.6). Osteons permit crack initiation but prevent crack growth. A weak cement line interface allows for easy crack initiation (Currey, 1962; Burr et al., 1988). The cement line, however, is more ductile then the surrounding bone matrix and the crack stress decreases when the crack passes from a ductile interface to a more stiff material. Martin and Burr (1989) also found that an increasing number of osteons increased the ability of the bone to resist crack propagation.

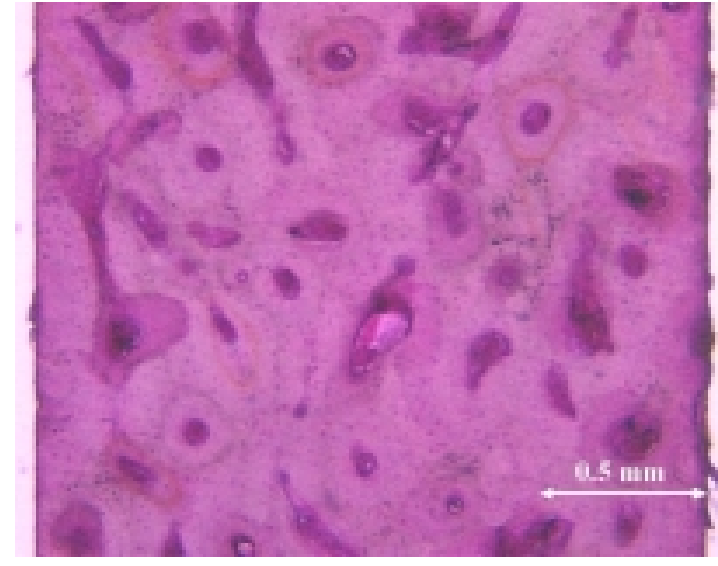

(A)

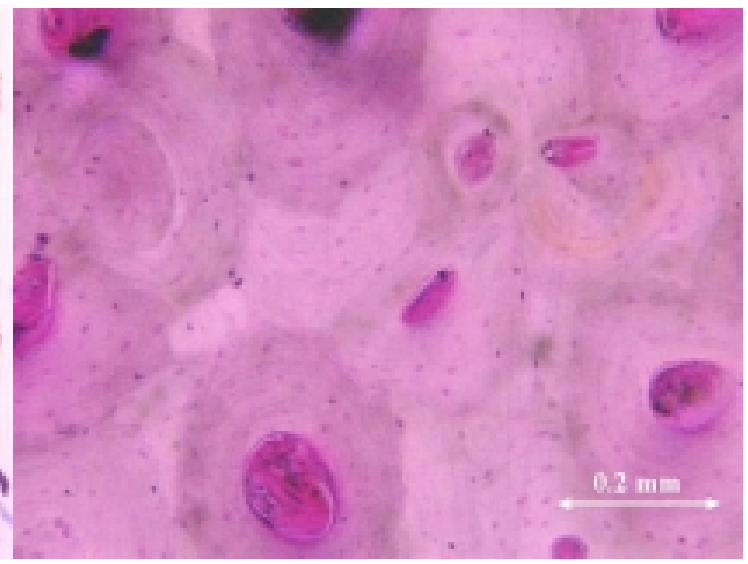

(B) 


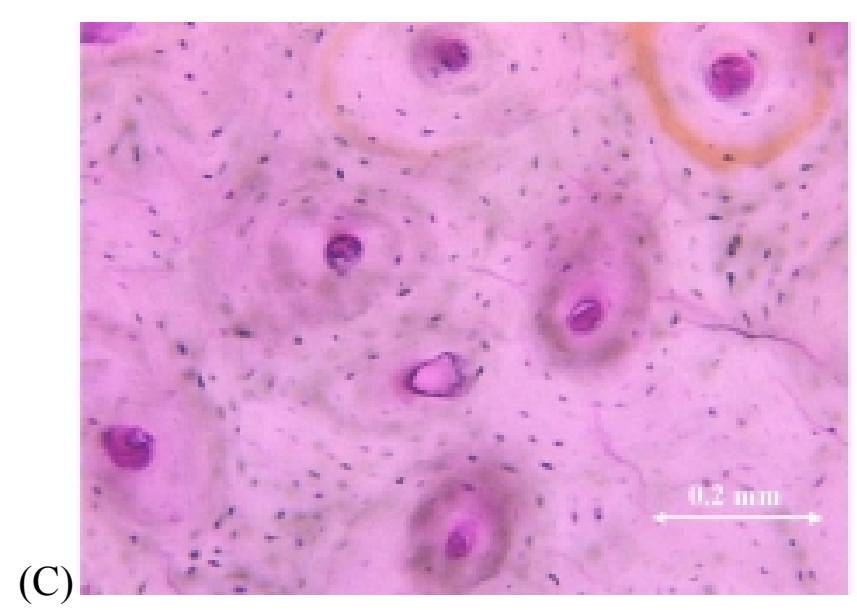

Figure 1.6 Cross-sections of human cortical bone stained with hematoxylin and eosin. (A) Haversian canals are dark stained regions within osteons. (B) The osteons have a cement line surrounding them that separates them from the bone matrix. (C) Microcracks in the bone matrix can be seen on the middle right.

\subsection{Clinical Significance}

Total hip arthroplasty is a surgical procedure to replace the hip joint. It involves inserting an implant into the shaft of the femur. There are two common types of hip arthroplasty used to repair hip fractures: uncemented and cemented implant fixation. One method of uncemented implant fixation is a press-fit of the implant into the bone canal. Press-fit fixation relies on the implant applying a radial force to the proximal femur resulting in primarily tensile hoop and compressive radial strain. The interference fit holds the implant in place until new bone growth occurs (Blaha 1989). Human bone is known to experience creep, which can influence implant stability.

The other type of arthroplasty involves cementing the implant into the cortical bone shaft of the femur. Cemented stems are done more often in elderly patients who have thin cortical bone or poor bone quality. A press-fit implant may better suit a younger, active patient with good bone quality and a thick cortical shell.

Clinical success of a hip arthroplasty is determined by the patient's ability to carry on a normal life without pain caused by their hip. Pain and limping after hip surgery is caused mainly by clinical loosening of the hip implant. When loosening occurs the implant no longer has stable fixation. The initial fixation of the implant can be achieved 
with a press-fit of the prosthesis in the femoral canal (Engh et al., 1987; Butler et al., 1988; Blaha et al., 1993).

A successful press-fit implant achieves and maintains stability. Stability is defined by motionless contact between the host bone and the implant. This interface between the two surfaces remains motionless throughout loading of the hip. Short-term stability first occurs during the initial implantation of the stem in the femoral canal. Intermediate stability occurs after the implant re-establishes itself when load is applied to the hip during normal use. Long-term stability occurs in the many years following the surgery. The osseointegration that occurs due to a stable implant ensures the long-term stability (Blaha, 1998).

Once the prosthesis has been inserted the implant taper guides the stem to seat and wedge itself into the canal. The viscoelastic behavior of the cortical bone releases the initial fit around the implant because of creep. This motion can contribute to the stability of the implant system if it occurs before bone growth has begun, around 6 to 8 weeks, and causes the implant to again re-establish a press-fit. It is also thought that motion before bone growth to the implant can inhibit biological fixation and promote bone resorption (Schneider et al., 1987). If bone ingrowth does occur, stability of the implant can be achieved. An essential prerequisite for the implant, therefore, is long-term preservation of the host bone. Little is known, however, of the role of the creep behavior of bone in achieving stability.

Long-term success or stability of a press fit implant is highly dependent on the initial fixation of the implant into the bone (Blaha et al., 1993). Another component of long-term fixation is long-term preservation of the host bone after implantation. Without healthy bone to hold the implant in place, failure of the uncemented system will ensue. A clinical and radiographical study of a type of press-fit prosthesis proved favorable biomechanical compatibility with minimal bone loss which the authors attribute to the initial stability provided by a true "press-fit" of the tapered implant (Blaha et al., 1993).

Femoral hip implants inserted directly into the intramedullary canal of the femur have experienced widespread clinical success, however, there have been proximal femur fractures during stem insertion. They occur because high circumferential stresses are generated in the bone as an oversized stem is forced into the proximal canal. Femoral 
fractures due to stem insertion is most likely affected by (1) the size mismatch between the canal and implant (2) reduction of cortical bone integrity during reaming to accommodate the size of the stem, or (3) inadequate bone material quality. Under normal physiological conditions femoral bone does not experience the magnitude of circumferential stress that may occur during implant insertion (Jasty et al., 1993). As a result the bone may not be able to endure the radial forces and circumferential (hoop) stress in the cortex, especially when the cortex is compromised.

\subsubsection{Clinical Hip Fracture}

The proximal region of the femur is a common site of fracture in the elderly and can lead ultimately to total hip replacement surgery. There are approximately 1.3 million fractures per year in the United States (NIH, 1984). Approximately 200,000 to 250,000 of these fractures are hip fractures (Brody, 1985; Babbitt, 1994; Brainsky et el., 1995). The incidence of hip fractures increases with age and the risk of sustaining a hip fracture is as high as 32\% (Melton III, 1988; Cummings et al., 1989). As the elderly population grows, the number of hip fractures may double or triple in the middle of the 21 st century.

For those who sustain hip fractures, $12-20 \%$ die as a result of complications caused by the hip fracture itself (Cummings et al., 1985; 1990; Farmer et al., 1984; Holbrook et al., 1984; Magaziner et al., 1989; Melton III and Riggs, 1983; Perry III and Morley, 1992; Gallagher et al., 1980; Lewinnek et al., 1980; Riggs and Melton III, 1986). For those who survive the initial hip fracture and the reconstruction surgery, $50 \%$ lose their independence and functional ability (Cummings et al., 1985; Farmer et al., 1984; Holbrook et al., 1984; Magaziner et al., 1989; Melton III and Riggs, 1983; Perry III and Morley, 1992; Kellie and Brody, 1990).

Approximately 127,000 total hip replacements are conducted each year to repair hip fractures. About $15 \%$ of these operations are not successful. Failure of this operation causes both pain and expense for the patient. A second attempt for a hip replacement has an even higher failure rate (Courtney et al., 1995; Graves, 1994). The cost of an average individual hip fracture is $\$ 29,800$ (Brainsky et al., 1995) and the annual total cost of hip fractures is between $\$ 7$ and 10 billion in the U. S. By the year 2020, the total cost may be as high as $\$ 60$ billion (Edwards and Perry III, 1994; Babbitt, 1994). 


\subsubsection{Intraoperative Fractures}

Intraoperative fractures can occur during uncemented total hip arthroplasty surgery resulting from preparation of the femoral canal or during final impaction of the implant. Such fractures may occur as a result of hoop stresses induced by the insertion of the implant (Kyle, 1999). Fitzgerald et al. (1988) reported 40 intraoperative fractures out of 630 arthroplasties (6.3\%) and all but two fractures were detected during surgery. Surgeries in which the PCA implant was used resulted in a fracture rate of $5.9 \%$, of the $\mathrm{HG}$, Bias and Omnifit implants, a fracture rate of $4.2 \%, 4.5 \%$ and $15.2 \%$ was found to result in fracture, respectively. Schwartz et al. (1989) reported 39 fractures out of 1318 procedures $(3 \%)$ done with the uncemented anatomic medullary locking prosthesis, however only half were detected during the procedure. All 39 were assumed to occur during the procedure, obscured by overlapping soft tissue (Jasty et al., 1993), thus labeled intraoperative fractures. Kavanagh (1992) reported an incidence of fracture of $2 \%$ using the PCA implant when the same sized rasp as the implant was used (Kavanagh et al., 1989) and a 20\% incidence with the Omnifit implant when used with an undersized rasp (Cabanela, 1990).

Besides the clinical research regarding fractures during surgery, there are in-vitro studies that also suggest assembly strains of uncemented hip replacement insertion may result in fracture. Jasty et al. (1993) measured the assembly strains while preparing the femur for uncemented hip arthroplasty. Preparing the canal for a press-fit implant $0.5 \mathrm{~mm}$ larger than the canal resulted in strains below that which would be expected to cause fractures. Insertion of the implant caused hoop strains as high as $2400 \mu \varepsilon(700-2400 \mu \varepsilon$, $1160 \pm 340 \mu \varepsilon$ mean of seven bones tested) in the proximal femur. Strains in the distal femur ranged from 100-1100 $\mu \varepsilon$. Peak tensile hoop strains, 2400-6000 $\mu \varepsilon$, were recorded before the development of a fracture.

Reid et al. (1995) conducted nonlinear two dimensional finite element analyses modeling the interference fit between bone and a press-fit implant. Circumferential tensile strains, as high as $32000 \mu \varepsilon$ for a $0.5 \mathrm{~mm}$ press-fit were observed. These strain magnitudes were high enough to cause longitudinal fractures compared to the experimental research conducted by Jasty et al. (1993). 
The postoperative period includes any time after surgery. The vast majority of fractures reported postoperatively are the result of major trauma such as car accidents or falls. Other fractures recognized postoperatively, within days to weeks, often represent extensions of previously unrecognized intraoperative fractures or the result of unprotected stress risers in the bone (Kelley, 1994; Petty, 1991). Perhaps the appearance

of postoperative fractures is not always due to the failure of detecting an intraoperative fracture. Instead, fractures develop as a result of the time-dependent nature of cortical bone. High hoop and radial stresses may exist in the bone due to the implant and remain constant. The stress results in creep deformation and fracture at the inner cortex, which extends towards the outer cortex and also longitudinally. Once the fracture reaches the outer cortex and is of sufficient length, it may be detectable postoperatively.

Migrating fractures have been observed postoperatively, which develop from a minor crack at the time of surgery (Penenberg, 1998). These types of fractures may lead to revision surgery, which has a much higher failure rate due to sub optimal fixation or poor bone quality. Therefore, the prevention of these fractures would limit the overall morbidity of patients. Unrecognized fractures occur infrequently, however, due to the large number of procedures done every year, understanding the time-dependent nature of bone to prevent such fractures is necessary.

\subsubsection{Effect of Creep on Hip Arthroplasty and Stability}

Axial load applied to a tapered stem-type femoral implant used in hip arthroplasty causes a press-fit into the cortical bone. The press-fit stem applies radial forces to the inner surface of the bone that results in radial and circumferential stresses. The circumferential stress serves to hold the implant like a nail driven into wood (Figure 1.7). This stress state in bone may have a role in the reported radiographic evidence of adaptive bone remodeling associated with intramedullary femoral fixation following hip arthroplasty such as cortical hypertrophy or endosteal expansion with cortical thinning. In addition, the level of assembly strains may induce a response in bone that includes longitudinal fracture in the cortical bone (Reid et al., 1995; Jasty et al., 1993). 


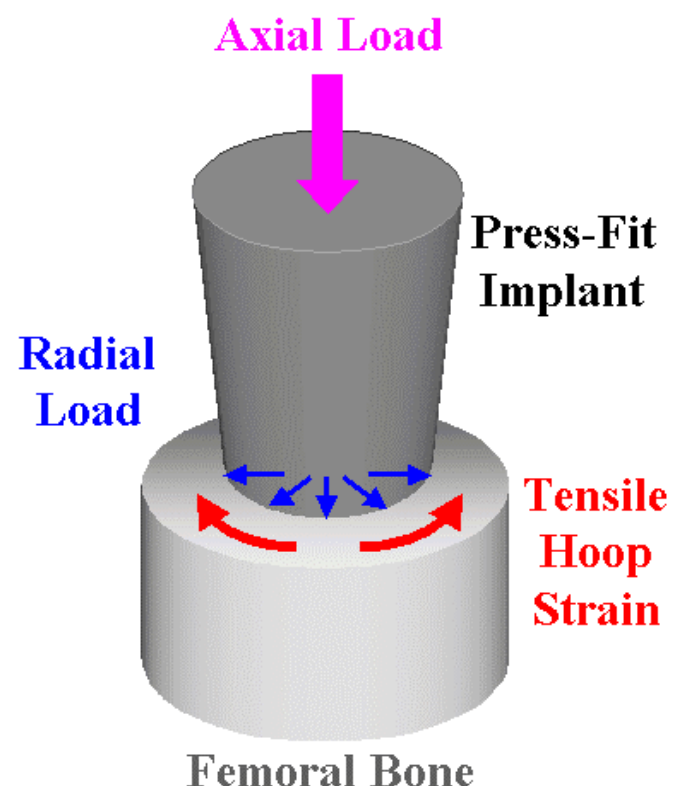

Figure 1.7 Radial forces from the press-fit implant result in tensile hoop strain in the bone.

The viscoelastic behavior of human femoral cortical bone may have clinical ramifications. The level of stress during a press-fit will induce a response in bone that includes one or more of the following: a purely elastic response, a viscoelastic response, and damage or plastic response leading to failure. The elastic response of the bone holds the implant in place achieving initial stability. The radial load from the stem causes a sufficient fit with the bone allowing the implant to remain in stable, motionless, contact (Blaha et al., 1991). A small radial load may cause deformation of the bone that will result in stable fixation, or equilibrium, where the implant does not subside further within the femoral canal. A large radial load may cause viscoelastic deformation that does not stabilize with time. Rather, a constant creep strain rate results in deformation leading to either excessive subsidence or failure of the bone. Defining the amount of stress which is the boundary between linearly increasing hoop creep strain and exponentially increasing strain is important. Understanding this type of bone behavior may provide insight on initial stability and instability for clinicians interested in the initial fixation of prosthetic implants. 


\subsubsection{Long Femoral Implants}

Primary hip arthroplasty generally involves implants that rely on the proximal femur for fixation. However, during revision surgery longer implants may be used. Long cemented and uncemented implants rely on bone more distal than during the original procedure. A major disadvantage is that stress shielding is more likely to occur in the proximal femur due to the more distal load transfer. Further revision surgeries are therefore more difficult due to resorption and thinning of the proximal femoral cortex. The increase in implant length, however, provides more surface area for fixation, relies on fixation well beyond the fracture site, relies on bone undisturbed during previous procedures, and bypasses weakened bone at the proximal femur as a result of bone resorption (Chang et al., 1999; Miller et al., 1982; Scott et al., 1975; Scheller and D’Errico, 1982; Turner and Emerson, 1982).

Bone for this study was taken from the mid to distal diaphysis of the human femoral shaft for two reasons. Bone from the proximal femur $(95 \mathrm{~mm}$ distal from the lesser trochanter) was used in a previous research project. Secondly, a consistently thick cortical shell was needed for this study in order to machine the bone specimens. Therefore, using bone specimens originating at the mid-diaphysis of the femur for the cylinders was a necessary limitation of the study.

\subsubsection{Uncemented Hip Implant List}

In an attempt to determine the role of interference fit in the success of cementless hip implants the following list were researched: the CLS stem, the Zweymuller stem, the Omnifit stem, the Identifit stem, the anatomic medullary locking (AML) stem, the anatomic porous replacement (APR) stem, the porous-coated anatomic (PCA) stem, and the HGP stem. The goal of this literature review was to compare the clinical success or failure of the specific cementless system and the recommended interference fit between the oversized stem and the prepared femoral canal.

It is generally accepted from clinical reports that success of uncemented stems improves with the press-fit achieved at surgery, thus initial stable fixation is important (Poss et al., 1988a; Bombelli and Santore, 1984; Engh, 1983; Morscher, 1983; Ring, 1978). The degree of fit refers to the amount of contact between the implant and bone. 
Maximum contact, especially proximally (Robertson et al., 1988; Manley and Averill, 1989), results in more normal strain patterns, reduces micromotion, reduces pain, and assists bone growth to further stabilize the implant (Poss et al., 1988a; Robertson et al., 1988; Itami et al., 1983; Morscher, 1983; Poss et al., 1988b; Ring, 1978; Ring, 1983; Walker et al., 1987). Poor proximal fit reduces the contact area between the stem and bone and increases the contact stresses (Manley and Averill, 1989).

Terms are used in the literature to describe the type of size difference between the implant and prepared femoral canal. The term precise fit or line-to-line fit describes the stem being the exact size of the prepared femoral canal. The term press-fit describes an oversize stem being fit into the prepared femoral canal. Determining the size of the stem relative to the prepared femoral canal for press-fitted stems is difficult based on the literature which rarely states the exact size difference. To determine the recommended difference in size for press-fit stems, sales reps for the manufacturing companies were contacted. Table 1.1 shows the recommended oversized dimension of the stem relative to the prepared femoral canal.

Table 1.1 List of uncemented stems and recommended size difference between stem and femoral canal.

\begin{tabular}{|c|l|}
\hline $\begin{array}{c}\text { Implant } \\
\text { name }\end{array}$ & \multicolumn{1}{c|}{ Size Difference } \\
\hline CLS & Implant is anchored into trabecular bone proximally with fins \\
\hline Zweymuller & Implant is the same size as the final broach \\
\hline Omnifit & Implant is $0.5-1 \mathrm{~mm}$ larger than the final broach \\
\hline Identifit & $\begin{array}{l}\text { Same size reported in literature (Robinson and Clark, 1996) } \\
\text { Implant is custom made to be } 0.5 \mathrm{~mm} \text { larger than final broach (sales rep) }\end{array}$ \\
\hline AML & Implant is 0.5mm larger than the final broach \\
\hline APR & $\begin{array}{l}\text { Implant is same size proximally } \\
0.5-1 \mathrm{~mm} \text { larger along midsection } \\
0.1 \mathrm{~mm} \text { larger than final broach distally }\end{array}$ \\
\hline PCA & Implant is 1mm larger than the final broach \\
\hline HGP 1 & $\begin{array}{l}\text { Implant is } 0.5 \mathrm{~mm} \text { larger than final broach proximally } \\
\text { Same size distally }\end{array}$ \\
\hline ATH & Implant is the same size as the final broach \\
\hline
\end{tabular}


The CLS system appears to be generally accepted as clinically successful with the most common disadvantage being the incidence of thigh pain. Spotorno et al. (1993) reported a mean postoperative Harris Hip Score of 94 for the first 300 CLS patients at the five to eight year follow up after surgery. $83 \%$ of the patients reported no pain and there was only a $1.6 \%$ mechanical failure rate. After an average of 5 years, $7.6 \%$ of 79 implants were reported as loose in one study by Ellis et al., 1997. Robinson et al. (1996) reported that after a mean of 6 years, $92 \%$ of the hips were rated good or excellent with a mean Harris Hip Score of 92. Blasius et al. (1993) reported that 91\% of 1830 cases were excellent or good with stem loosening in less than $1 \%$ after 2 years. Another study, 7.8 years after surgery, found good functional scores in more than $90 \%$ of 145 cases (Bulow et al., 1996). Thigh pain was found in only a single case. Blaha et al. (1991) reported, after 4 to 7 years, only $1 \%$ of the patients had pain 12 weeks after surgery.

The Zweymuller system was reported to be very successful. Zweymuller (1986) reported on the first 136 cases 3 to 5 years after surgery. Patients were either very satisfied or largely satisfied in $99.3 \%$ of the surgeries and $75 \%$ had no postoperative complaints. Five years after surgery, 96 stems had an average Harris score of 87.5 (Eyb et al., 1993). Only a single stem subsided within the first year and was stabilized. Huo et al. (1995) reported $98 \%$ of 46 hips were rated good or excellent clinically with no loosening reported.

The Omnifit system also appears to be successful with the most common disadvantage being the incidence of proximal femoral osteolysis. Hellman and Capello (1996) reported $96 \%$ of 79 hips with the absence or mild thigh pain after an average of 101 months. The revision rate was $2.5 \%$ and the mechanical failure rate was $3.8 \%$. Onsten et al. (1996) reported on 30 stems and found early fixation comparable with that of the cemented Charnley prosthesis. Hellman et al. (1999) expressed serious concern about the incidence of proximal femoral osteolysis, $37.5 \%$, in a study of 76 hips. $2.6 \%$ had aseptic loosening and 3.9\% had thigh pain. After 40 months, all stems achieved stable fixation. In a comparative study, the Omnifit stem produced inferior results to the HGP and PCA one year after surgery (Daum and Uchida, 1992).

The Identifit system did not achieve satisfactory stability and the common disadvantages were the lack of stability, excessive subsidence and pain. Robinson and 
Clark (1996) report results on 53 custom molded stems 30 months after surgery. Although the stem was able to achieve a high percentage of femoral canal fill, $17 \%$ required revision surgery. Of the remaining stems, the Harris score was $83,20 \%$ had moderate to severe thigh pain, $65 \%$ had stem subsidence, and $50 \%$ had a severe limp. Mathur et al. (1996) reported a high incidence of complications for 67 hips. $73 \%$ had subsidence of $2 \mathrm{~mm}$ or more and $25 \%$ had early postoperative complications.

The anatomic medullary locking (AML) hip system has achieved some success despite reports of stress shielding, difficulty with stem removal (Engh and Culpepper, 1997), and reported pain. Bugbee et al. (1997) reported on 44 stems that had at least 10 years of follow up data. $86 \%$ had no or mild pain, $42 \%$ had less pain than they had preoperatively and 41\% were satisfied with their results. Engh et al. (1987) reported that of the 195 stems which were classified as having a press-fit at the isthmus, 93\% appeared radiographically to have bony ingrowth. Of the stems which did not achieve a press-fit $69 \%$ were classified as having bony ingrowth. Incidence of pain and limp was much lower when a press-fit or bony ingrowth was achieved. The use of larger stems resulted in an increase in bone resorption (Engh and Bobyn, 1988), of 121 stems with canal filling, $4.1 \%$ had bone resorption as opposed to 290 stems without canal filling which had $24.1 \%$ resorption. Haddad et al. (1990a; 1990b) reported on 64 stems which had an average Harris score of 80.7 after surgery and thigh or groin pain in $30 \%$ of the cases. In a comparative study of 270 stems followed for 2 to 8 years, Hwang and Park (1995) reported Harris scores of 93, 93 and 91 for AML, PCA and HGP, respectively. No loosening was found in the AML and HGP stems but four PCA stems experienced aseptic loosening. Pain was reported in 17\%, 21\% and 19\% of the AML, PCA and HGP stems, respectively. Sugiyama et al. (1992) compared the line-to-line fit to a press fit insertion of the AML stem. The line-to-line group did not achieve good rotational fixation. The pressfit group had significantly less loosening and superior rotational stability with the extent of porous coating not having a significant effect. Bony ingrowth, such as dense corticalcancellous bone, assists in achieving fixation of the implant (Sugiyama et al., 1994; Whiteside et al., 1993).

The first anatomic porous replacement (APR-I) hip had an unacceptable failure rate with the common disadvantage of the loss of fixation of $70 \%$ after 6.7 years (Dorr et 
al., 1997). APR-II had much better results (Dorr and Wan, 1996). 148 stems were followed for 2 to 5 years. $82 \%$ had excellent results and $14 \%$ were good.

The porous coated anatomic (PCA) hip system appears to have clinical success however complications have been encountered including thigh pain, osteolysis and loosening. The PCA stem had similar success when compared to the AML. Haddad et al. (1990a; 1990b) reported an average postoperative Harris score of 83.8 for the PCA as compared a score of 80.7 for the AML stem. Both stems had thigh or groin pain in $30 \%$ of the cases. Dodge et al. (1991) reported an average Harris score of 90.5 for 35 cases an average of 37 months after surgery. Thigh pain occurred in $20 \%$ of the cases but the stems remained stable. Knight et al. (1998) reported a mean postoperative Harris score of 91.3 at a minimum follow-up of 5 years. $88 \%$ of the cases were rated good or excellent yet $30 \%$ reported moderate to severe thigh pain and $9 \%$ had stem subsidence. Less encouraging results were reported by Kim et al. (1999) on 116 hips followed for a minimum of 10 years. The average postoperative Harris score was 87 at 11 years. $65 \%$ of the hips were rated excellent, 9\% were good and 30\% were poor with a revision rate of the femoral component of 9\%. After 11 years, femoral osteolysis was reported in 59\% cases, and $28 \%$ of the cases still reported thigh pain which was found to be the result of aseptic loosening. Kim et al. (1999) state they have abandoned the use of this stem due to the complication rate.

The Harris-Galante Porous (HGP) system appears to have mixed results with common disadvantages being pain, lysis and loosening. de Nies and Fidler (1996) reported poor results for 57 hips at a mean of 3.4 years after surgery along with a Harris score of 81 . Only $60 \%$ were rated as excellent or good, thigh pain occurred in $52.6 \%$ and loosening occurred in 17.5\%. For a similar follow up time, 3.5 years, Woolson and Maloney (1992) reported 88\% of 69 hips rating excellent with a Harris score of 90 or more. Only $10 \%$ of the hips were unstable. Significant stress shielding was observed in $16 \%$ of hips and lysis in $22 \%$. Femoral lysis was also reported as a major complication, in $31 \%$ of 94 stems, by Smith and Harris (1995) after 4.4 years follow-up. Loosening was found in $15 \%$ of stems. $94.3 \%$ of 149 hips were rated as excellent and 5.7\% had good results (no fair or poor ratings) reported by Tong et al. (1993) after a mean of three years after surgery. $81.4 \%$ had stable bone ingrowth fixation. After an average of 5.5 years 
after surgery, $87 \%$ of 121 hips were rated as excellent or good (Martell et al., 1992) but 9.1\% had loosening. 10 intraoperative fractures were observed. After an average of 7.3 years follow-up, Saito et al. (1997) reported "excellent" results for a study of 68 hips. $17.6 \%$ of the cases reported thigh pain which lessened; the mean hip score was 91.2 at last follow-up. Although 93\% of 100 stems were rated as excellent or good, Lachiewicz et al. (1992) expressed concern over subsidence of $3 \mathrm{~mm}$ or more in $16 \%$ of hips. $80 \%$ of hips were reported to have no pain during follow-up after 2 to 5 years. At an average 5year follow-up of 82 stems, Kim and Kim (1992) reported poor clinical results; $28 \%$ of the hips experiencing thigh pain and $10 \%$ experienced loosening and the average Harris score was 83.3. In a 4 to 8 year follow up study of 100 stems, Petersilge et al. (1997) reported a mean Harris score of 92 , moderate thigh pain in $6 \%$ of hips, $3 \%$ unstable stems and a subsidence frequency of $22 \%$. In a 10 year follow-up study of 77 stems by Clohisy and Harris (1999) reported a high incidence of femoral lysis, in 60\% of hips, and $25 \%$ were revised or loose.

Based on this literature it was difficult to draw conclusions comparing the success or failure of stems to the difference in size between stem and the prepared femoral canal. Other factors including stem geometry, patient parameters such as age, the surgeon's experience, and how closely the surgeon follows the recommended instructions given for the implant most likely contribute to clinical success and failure. 


\section{CHAPTER 2 \\ VISCOELASTICITY AND CREEP BEHAVIOR}

\subsection{Definitions}

Viscoelastic materials range from classical viscous fluids such as water and air, to elastic solids such as rubber and metal. In between these extremes, materials may combine viscous and elastic properties in any proportion. Environmental conditions also affect the viscous and elastic behavior. A plastic material may occupy a position between the middle of the two extremes or near the elastic extreme under different temperatures.

In order to understand the nature of a material's creep behavior, it is necessary to understand different material responses to loading. A response characterized by a nonlinear stress-strain curve may either retain permanent strain or return to its unstrained state after complete unloading (Boresi et al., 1993).

If a material is loaded in tension at a sufficiently slow rate and at a low stress the relationship between stress and strain is linear elastic, a straight line (Figure 2.1). As the load increases the relationship between stress and strain may become nonlinear (Boresi et al., 1993).

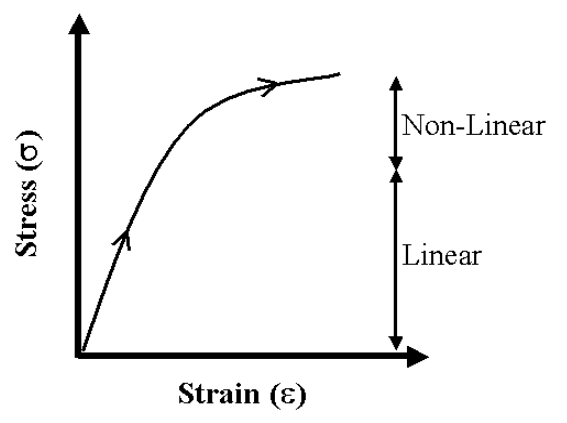

Figure 2.1 A stress strain curve displaying linear and non-linear behavior.

The elastic or plastic nature of a material is determined by the materials response during unloading. If the unloading path follows the loading path, the loading is reversible and the material is elastic; all deformation occurring during loading is recovered during unloading (Figure 2.2a). If the unloading path does not follow the loading path the material exhibits plastic behavior; deformation is not completely recovered during unloading (Figure 2.2b) (Boresi et al., 1993). 


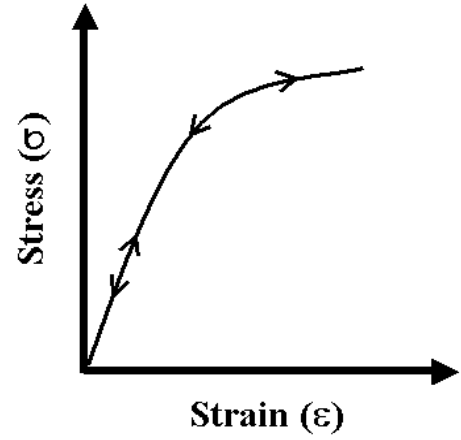

(a) Elastic Response

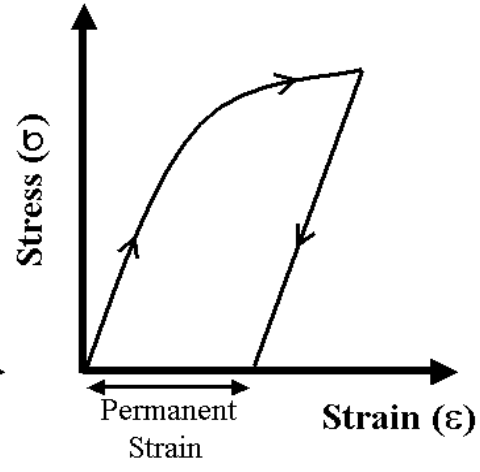

(b) Plastic Response

Figure 2.2 (a) The elastic response where deformation is recovered and (b) the plastic response where deformation is not completely recovered.

Materials influenced by the strain or stress rate are considered to be timedependent and exhibit viscous behavior. A viscoelastic material experiences timedependent and elastic behavior. An elastic action occurs upon loading followed by a slow continuous increase in strain at a decreasing rate (Figure 2.3a). Upon complete unloading, an initial elastic recovery is followed by a continuously decreasing strain, and over time the material returns to its original undeformed state (Lockett, 1972; Findley et al., 1976; Boresi et al., 1993). A viscoplastic material will also continue to recover deformation after the load is removed, however, some permanent strain will remain (Figure 2.3b) (Boresi et al., 1993).

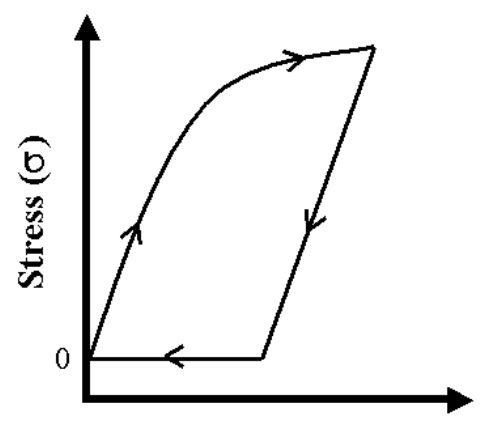

Strain ( $)$

(a) Viscoelastic Response

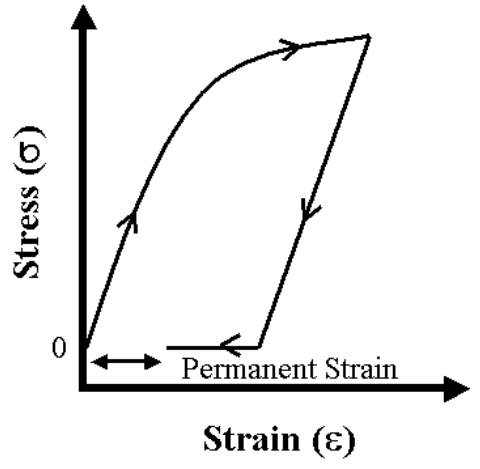

(b) Viscoplastic Response

Figure 2.3 (a) Viscoelastic material response where deformation is recovered with time and (b) a viscoplastic material response where deformation is not recovered in time. 
An example of a metal exhibiting viscoelastic behavior occurs when a highvelocity projectile hits a sheet of steel. The steel deforms at the point of impact similarly as water deforms when a stone is dropped in a pond. The material at and near the impact point behaves more like a viscous fluid than an elastic solid. "Silly putty" is another example, at slow loading rates the putty acts as a very viscous solid, however the putty may bounce like a ball when dropped. So in the case of steel, the material acts like an elastic material at slow loading rates and like a viscous fluid at high loading rates, whereas the putty acts like a viscous fluid at slow loading rates and elastically at high loading rates (Lockett, 1972). A biological example of viscoelastic behavior is the rate dependent load-displacement response of hard and soft tissue (Cowin, 1989; Fung, 1981; McElhaney, 1966).

Two types of viscoelastic behavior include creep and stress relaxation. Stress relaxation involves a constant deformation while the load required to maintain that deformation decreases (Figure 2.4a). An elastic material would require a constant load to maintain the constant deformation. A viscoelastic material subjected to a constant deformation will relax so that stress gradually decreases with time (Findley et al., 1976). Relaxation may be thought of as internal creep where stress is decreased and redistributed without significantly deforming the boundaries (Byars et al., 1983). Creep is a viscoelastic response of a material and is defined as time-dependent strain under a sustained load (Boresi et al., 1993) (Figure 2.4b). After an instantaneous elastic response, deformation continues to take place despite a constant load.

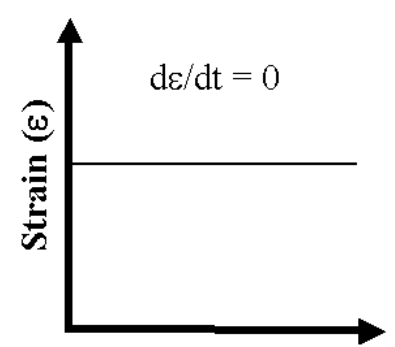

Time

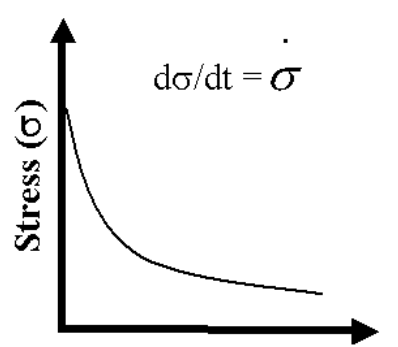

Time

(a) Relaxation 


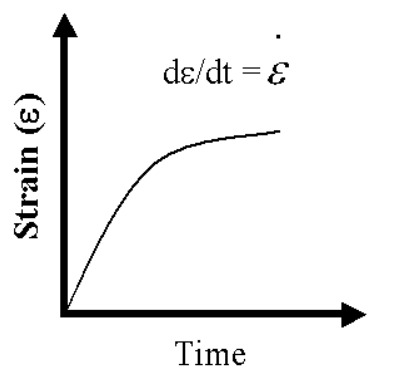

Time

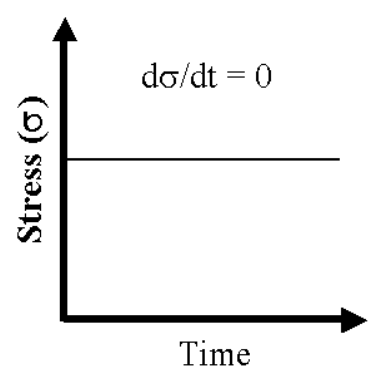

(b) Creep

Figure 2.4 (a) At a constant strain a viscoelastic material may require less stress to maintain the deformation over time or relax. (b) A viscoelastic material may creep, or deform under constant load over time.

Creep behavior is a function of stress and temperature and can be described with a strain-time plot with three distinct stages: primary, secondary, and tertiary (Figure 2.5). The primary creep stage begins with the "instantaneous" strain, $\varepsilon_{0}$, at the time of load application and the creep strain rate decreases.

Once the strain rate $(\mathrm{d} \varepsilon / \mathrm{dt})$ decreases to a minimum, relatively constant value, the secondary creep stage begins. During this phase, the minimum strain rate remains constant. The average value of the creep strain rate during secondary creep is called the minimum creep rate. The slope during the second stage of creep may reach a zero slope so that the strain reaches an equilibrium or long-time constant value. The curve may also approach a constant non-zero slope when strain increases in a linear time-dependent manner (viscous fluid behavior) (Lockett, 1972).

The third stage occurs during constant load tests. When the strain rate begins to increase, the final, tertiary, stage occurs where the strain rate increases until the material fails (Dieter, 1986). This third stage occurs as there is an effective reduction in crosssectional area of the specimen because of necking or internal void formation. Thus if creep is maintained for a sufficiently long time, excessive deformation or fracture (creepfracture) occurs (Boresi et al., 1993). 
(A)

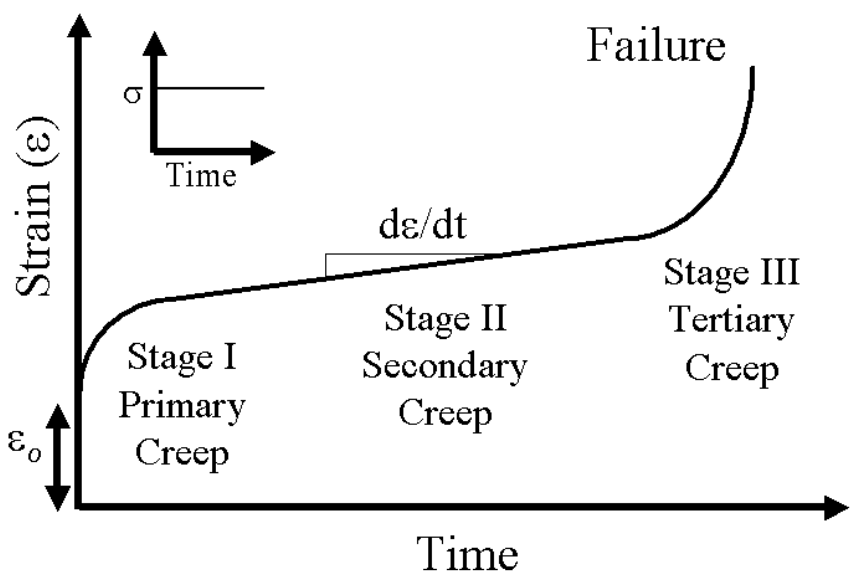

(B)

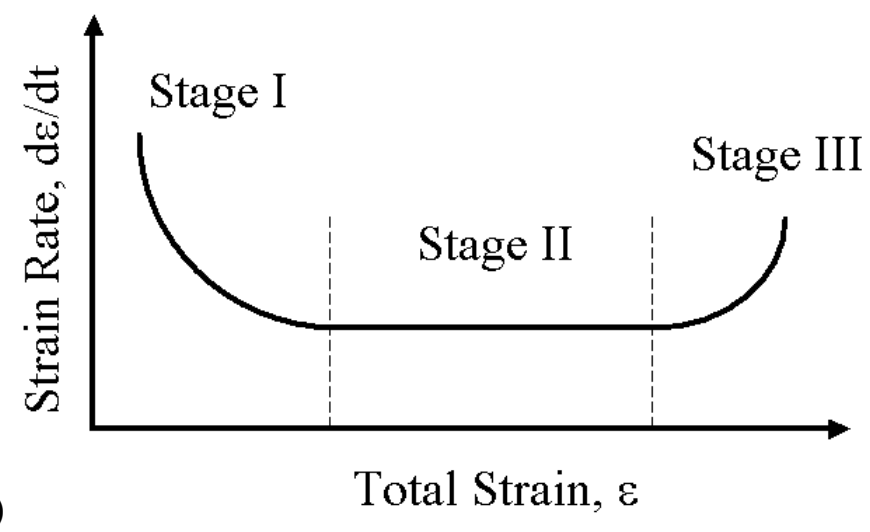

Figure 2.5 (A) The three stages of creep and (B) strain rate. During primary creep, the strain rate decreases after an initial load is applied. The minimum strain rate is maintained during secondary creep. Finally, the strain rate increases until failure. The shape of all three stages will depend on load rate, load level and load orientation (tension vs. compression).

Total strain at any instant in time of a linear material is represented by the sum of instantaneous elastic strain, $\varepsilon_{\mathrm{e}}$, and creep strain $\varepsilon_{\mathrm{c}}$ :

$$
\varepsilon=\varepsilon_{\mathrm{e}}+\varepsilon_{\mathrm{c}}
$$

Strain rate is found by differentiating $\varepsilon$, note that $\varepsilon_{\mathrm{e}}$ is a constant:

$$
\frac{d \varepsilon}{d t}=\frac{d \varepsilon_{c}}{d t}=\dot{\varepsilon}
$$

After the load is removed from a viscous material, a delayed recovery path is followed, where instantaneous elastic recovery is followed by a gradual recovery (Lockett, 1972) (Figure 2.6). Full recovery of the deformation is dependent on the type of 
material, such as plastics, metals or fluids (Findley et al., 1976) and applied load magnitude. Upon complete unloading, an elastic material will immediately return to its undeformed state. A viscoelastic material will follow a delayed recovery path consisting of an instantaneous elastic component followed by gradual recovery (Lockett, 1972).
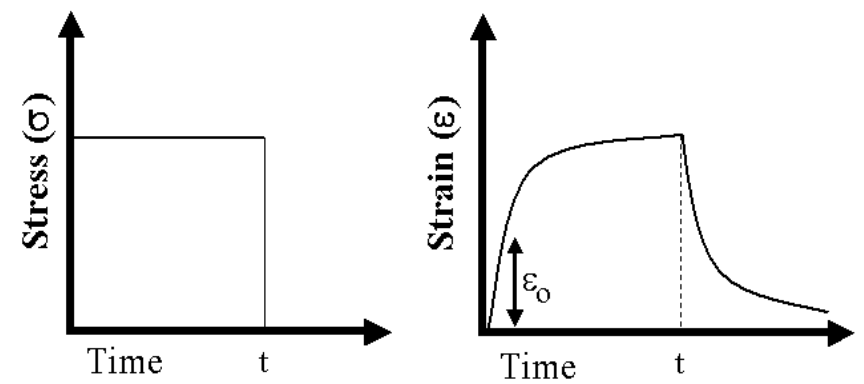

Figure 2.6 For a viscous material, the instantaneous elastic strain, $\varepsilon_{0}$, is followed by continued deformation during the constant load. Once the load is removed, an initial elastic recovery is followed by gradual recovery. Some permanent strain may remain depending on the load and the material.

A creep test is generally considered to be a constant stress experiment, however, constant load tests are usually performed. During uniaxial constant tensile loading, the specimen elongates and decreases in cross-sectional area, necking, therefore resulting in an increase in axial stress and exhibiting the observable third stage of creep (Dieter, 1986). Constant stress tests usually result in an unobservable third stage of creep and also great delays before onset of the third stage of creep because the geometry loses less surface area (Findley et al., 1976).

\subsection{Thick Wall Cylinders}

The general equations for the hoop and radial stress at any point on a thick walled elastic cylinder with open ends and in the absence of temperature change are (Timoshenko and Goodier, 1970):

$$
\begin{aligned}
& \sigma_{\theta}=\frac{-r_{i}^{2} r_{o}^{2}\left(p_{o}-p_{i}\right)}{r_{o}^{2}-r_{i}^{2}} \frac{1}{r^{2}}+\frac{p_{i} r_{i}^{2}-p_{o} r_{o}^{2}}{r_{o}^{2}-r_{i}^{2}} \\
& \sigma_{r}=\frac{r_{i}^{2} r_{o}^{2}\left(p_{o}-p_{i}\right)}{r_{o}^{2}-r_{i}^{2}} \frac{1}{r^{2}}+\frac{p_{i} r_{i}^{2}-p_{o} r_{o}^{2}}{r_{o}^{2}-r_{i}^{2}}
\end{aligned}
$$


where $p_{o}$ and $p_{i}$ is the pressure on the outer and inner surface, respectively, $r_{i}$ and $r_{o}$ is the inner and outer radius, respectively, and $\mathrm{r}$ is a position along the radius of the cylinder cross-section. For the special case when $\mathrm{p}_{\mathrm{o}}$ is zero:

$$
\begin{aligned}
& \sigma_{\theta}=\frac{r_{i}^{2} p_{i}}{r_{o}^{2}-r_{i}^{2}}\left(1+\frac{r_{o}^{2}}{r^{2}}\right) \\
& \sigma_{r}=\frac{r_{i}^{2} p_{i}}{r_{o}^{2}-r_{i}^{2}}\left(1-\frac{r_{o}^{2}}{r^{2}}\right)
\end{aligned}
$$

Radial stress is always a compressive stress that is greatest at the inner surface $\left(\sigma_{\mathrm{r}}=\mathrm{p}_{\mathrm{i}}\right)$ and zero on the outer surface $\left(r=r_{0}\right)$. Hoop stress is always a tensile stress and is its maximum value also at the inner surface. The cylinder in this study is assumed to be in a state of plane stress since no force is applied in the longitudinal direction yet axial deformation is allowed, therefore longitudinal stress, $\sigma_{\mathrm{z}}$, is zero.

An alternate assumption is that the bone exhibits creep deformation that is governed by the Norton-Odqvist flow rule. In this case the following equations are used to solve for hoop and radial strain rate and hoop and radial stress for a thick walled cylinder with internal pressure (Skrzypek and Hetnarski, 1993):

$$
\begin{aligned}
& \dot{\varepsilon}_{\theta}=-\dot{\varepsilon}_{r}=\frac{k}{2} 3^{(1+n) / 2}\left(\frac{p_{i}}{n}\right)^{n}\left[\left(\frac{r_{o}}{r_{i}}\right)^{2 / n}-1\right]^{-n}\left(\frac{r_{o}}{r}\right)^{2} \\
& \sigma_{\theta}=\frac{p_{i}}{\left(\frac{r_{o}}{r_{i}}\right)^{2 / n}-1}\left[1-\left(1-\frac{2}{n}\right)\left(\frac{r_{o}}{r}\right)^{2 / n}\right\rceil \\
& \sigma_{r}=\frac{p_{i}}{\left(\frac{r_{o}}{r_{i}}\right)^{2 / n}-1}\left[1-\left(\frac{r_{o}}{r}\right)^{2 / n}\right\rceil
\end{aligned}
$$

where $p_{i}$ is internal pressure, $r_{o}$ and $r_{i}$ are the outer and inner radii, respectively, and the parameters $\mathrm{k}$ and $\mathrm{n}$ are the coefficient and exponent of the power law function. 


\subsection{Previous Investigations of Time-Dependent Bone Deformation}

\subsubsection{Viscoelastic Behavior of Longitudinally Oriented Bone Specimens}

Improving the success of total hip replacements may be aided by better understanding the behavior of human cortical bone. Specifically, the viscoelastic response in the transverse direction. The majority of our knowledge of the viscoelastic nature of bone has been studied by loading bone in the longitudinal direction. The following creep investigations of longitudinally and transversely oriented bone specimens are listed in Table 2.1 .

Wertheim (1847) and Rauber (1876) made the first observations of the viscoelasticity of human bone while determining measurements of the breaking strength. Smith and Walmsley (1959) reported on bone creep in tension and relaxation. They conducted tensile tests for 20 minutes on human cortical bone. Steady state creep was reached in 10 minutes and strain recovery was attained after 50 minutes of zero-load. They suggest that the viscoelastic effects may be the result of fluid motion within bone. Sedlin (1965) applied tensile load levels of 8.2, 16.3, 32.7, and 49.2 MPa in creep tests of human cortical bone. Creep rates of $146 \pm 2000 \mu \varepsilon, 557 \pm 347 \mu \varepsilon$ and $1629 \pm 1301 \mu \varepsilon$ were produced, respectively. Residual strains occurred when the specimens were unloaded, 5.6 $\pm 12.5 \mu \varepsilon 191 \pm 207 \mu \varepsilon, 974 \pm 840 \mu \varepsilon$, respectively. Smith and Keiper (1965) examined viscoelastic moduli of wet human bone using an electromagnetic transducer.

Currey (1965) examined cantilever bovine bone specimens for the effects of flexure on creep properties and anelasticity. A creep specimen was loaded for 10 days (a load was not specified, only that the specimen was not loaded into the plastic range) at room temperature in saline. An immediate deflection was measured upon initial loading and at the end of the ten days an increase of $33 \%$ of the original deflection was recorded. A plot of deflection versus log-time resulted in a curve concave up suggesting that an equilibrium deflection had not been reached. At the end of the ten-day loading period, the load was removed and an immediate recovery was followed by a continuous exponential recovery. After 100 days, most of the deflection had been recovered. Loading a single specimen with different weights showed that creep deflections were proportional to load. 
Table 2.1 Bone creep investigations with respect to type of loading and specimen geometry. Long. $=$ Longitudinally oriented specimen, Trans. $=$ Transverse, tangentially oriented specimen, rect. $=$ rectangular geometry, cyl $=$ cylindrical geometry

\begin{tabular}{|c|c|c|c|}
\hline Investigators & Bone & $\begin{array}{c}\text { Load } \\
\text { application }\end{array}$ & $\begin{array}{c}\text { Specimen } \\
\text { orientation and } \\
\text { geometry }\end{array}$ \\
\hline Smith and Walmsley (1959) & Human & Tension & $\begin{array}{l}\text { Long., unknown } \\
\text { geom. }\end{array}$ \\
\hline Sedlin (1965) & Human & Tension & Long., rect. \\
\hline Currey (1965) & Bovine & Bending & Long., stippled rect \\
\hline Bonfield and $\mathrm{Li}$ (1967) & Bovine & Torsion & Long., Trans., rect. \\
\hline $\begin{array}{c}\text { Utenkin and Sveschnikova } \\
\text { (1973) }\end{array}$ & Human & Compression & Long., Trans., rect. \\
\hline Crowninshield et al. (1974) & Bovine & Tension & $\begin{array}{l}\text { Long., Trans., } \\
\text { waisted rect. }\end{array}$ \\
\hline Knets and Vilks (1975) & Human & Tension & Long., rect. \\
\hline Melnis and Laizan (1978) & Human & Tension & Long., rect. \\
\hline Melnis et al. (1979) & Human & Tension & Long., rect. \\
\hline Lakes et al. (1979) & $\begin{array}{l}\text { Human, } \\
\text { Bovine }\end{array}$ & Torsion & Long., waisted cyl \\
\hline Lakes and Kat & $\begin{array}{l}\text { Human, } \\
\text { Bovine }\end{array}$ & Torsion & Long., waisted cyl \\
\hline Lakes and Saha $(1979,1980)$ & $\begin{array}{l}\text { Human, } \\
\text { Bovine }\end{array}$ & Torsion & , rect. \\
\hline Melnis and Knets (1981) & Human & Tension & Long., rect. \\
\hline Melnis et al. (1981) & Human & Tension & Long., rect. \\
\hline Carter and Caler (1983) & Human & Tension & Long., waisted cyl \\
\hline Park and Lakes (1986) & Human & Torsion & Long., rect. \\
\hline Fondrk et al. $(1988 ; 1990)$ & Human & Tension & Long., waisted rect. \\
\hline Caler and Carter (1989) & Human & $\begin{array}{c}\text { Tension } \\
\text { Compression }\end{array}$ & Long., waisted cyl \\
\hline Mauch et al. (1992) & $\begin{array}{c}\text { Bovine, } \\
\text { deer antler }\end{array}$ & Tension & Long., rect. \\
\hline Rimnac et al. (1993) & Bovine & Tension & Long., waisted rect. \\
\hline $\begin{array}{l}\text { Tanabe and Kobayashi } \\
\text { (1994) }\end{array}$ & Bovine & Compression & $\begin{array}{c}\text { Long., Trans., } \\
\text { Radial, cyl }\end{array}$ \\
\hline Pattin et al. (1995) & Bovine & $\begin{array}{c}\text { Tension, } \\
\text { Compression }\end{array}$ & Long., waisted cyl \\
\hline Jepsen et al. (1995) & Bovine & Torsion & Long., waisted cyl \\
\hline Vanscoy $(1996,1997)$ & Human & $\begin{array}{l}\text { Radial Stress, } \\
\text { Internal pressure }\end{array}$ & Long., hollow cyl \\
\hline Bowman et al. (1999) & $\begin{array}{l}\text { Human, } \\
\text { Bovine }\end{array}$ & Tension & Long., cyl \\
\hline
\end{tabular}


Utenkin and Sveschnikova (1973) loaded rectangular human femoral compact bone samples taken from different orientations with respect to the longitudinal axis of the original bone in compression. The specimens were compressed under constant stress for ninety minutes then the stress was removed and recovery was monitored for 60 minutes while strain was measured. Creep at the load levels of $50 \%$ and $90 \%$ of the elastic limits was dependent on the orientation of the sample however recovery did not.

Knets and Vilks (1975) conducted tensile creep tests on human tibia specimens at body temperature and humid but not wet conditions. Two stress levels, 35.7 and 53.6 $\mathrm{MPa}$, were applied for 100 minutes followed by a 100 minutes of zero-load. They concluded that bone is linearly viscoelastic up to $0.42 \sigma_{\text {ult. }}$ An equation was used to fit the creep strain data for a linear viscoelastic model.

Melnis and Laizan (1978) also tested tibia specimens under tensile load. Specimens were tested at body temperature and humid conditions at two load levels, 0.6 and $0.7 \sigma_{\text {ult }}$. The loading period lasted 200 minutes and was initially followed by a complete unloading and then zero-load for another 200 minutes. For the $0.6 \sigma_{\text {ult }}$ load level, an initial elastic deformation of $0.67 \%$ was measured and an equilibrium strain was not reached. A steady state creep rate of $18 \mu \varepsilon / \mathrm{s}$ existed over the last 80 minutes of load as did a residual deformation of $0.29 \%$ after 200 minutes of zero load. The specimens loaded to $0.7 \sigma_{\text {ult }}$ failed after 50 minutes, neither equilibrium nor steady state strain developed before failure. Two equations were provided to describe the creep and relaxation behavior. This creep model allows an equilibrium strain to be obtained.

Creep tests were done with human tibia longitudinal specimens at body temperature and humid conditions by Melnis et al. (1979). A series of constant loads, 0.2 to $0.7 \sigma_{\text {ult }}$ (or until failure), was applied for 200 minutes at increasing load increments of $0.1 \sigma_{\text {ult }}$. The specimens were unloaded for 1200 minutes between each loading cycle. The greatest creep strain was $0.17 \%$ during the maximum load of $113 \mathrm{MPa}$ and $72 \%$ was recovered during the unloading cycle. A steady state creep rate was observed, the maximum rate obtained during the highest load was $5.5 \mu \varepsilon / \mathrm{s}$. A power law equation modeled the nonlinear behavior for both the creep and relaxation deformation. The experimental results indicate the presence of three types of behavior depending on the load applied. Loads less than $0.2 \sigma_{\text {ult }}$ caused insignificant creep. Loads between $0.2 \sigma_{\text {ult }}$ 
and $0.5 \sigma_{\text {ult }}$ caused creep deformation during the 200 minutes of loading, which may reach equilibrium or progress until failure. Loads greater than $0.5 \sigma_{\text {ult }}$ cause creep deformation leading to failure.

Lakes and Saha (1979) applied constant torsional loads to bovine prism specimens for long periods of time, up to 8 weeks. The percent strain $2.6-8 \%$ was far greater than the $1.2 \%$ fracture strain reported for wet bovine bone in rapid torsional loading tests. Shear slippage was observed to occur at the cement line. They concluded that the process of viscous like slippage at the cement lines is a dominant mechanism responsible for large creep deformations.

Lakes et al., (1979) and Lakes and Katz (1979a; 1979b) conducted a series of investigations on viscoelastic properties of human cortical bone in torsion. Bone was shown to exhibit behavior that is thermorheologically complex and stress field dependent. In an attempt to better understand the viscoelastic response, they examined hypothetical relaxation mechanisms and derived a constitutive equation to describe the nonlinear torsional response of bone.

Lakes and Saha (1980) studied bovine and human bone longitudinal prisms in torsion. Recovery following removal of the load was almost complete for small loads, initial strain $0.15 \%$ or less; loads that correspond to the upper end of the normal physiological range. The creep compliance became large over long periods of time. The creep curves showed no sign of reaching an asymptotic value of compliance, even after loading for more than one month (Currey 1965). They found that over long periods of time, exceeding one month, bone experiences pronounced time dependent effects, final strains sometimes exceeding four times the initial strain. They felt that investigators assuming linear elastic theory for establishing theoretical stress analysis of bone-implant systems were justified for neglecting time-dependent effects from the time range of $10^{-3}$ to $10^{3} \mathrm{~s}$. However, neglecting time-dependent effects over a period of time exceeding this range was suspect.

The effects of age on the tensile creep properties of human tibia bone were studied by Melnis and Knets (1981). Longitudinal specimens were grouped into four age groups: I. 15-18 years, II. 30-35 years, III. 50-55 years, IV. 75-80 years, and tested in humid but not wet conditions at four load levels: $0.4,0.5,0.6$, and $0.7 \sigma_{\text {ult }}$ as in a previous 
study (Melnis et al., 1979). The oldest two age groups were not found to be different and were therefore combined. Significant differences in creep deformation were found between the following: groups I and II, groups I and III-IV, and groups II and III-IV. The greatest creep deformation was found for the youngest age group and decreased for older age groups. The creep rate also appears to be greater for the younger ages. An equation was used to describe the creep strain as a function of time, load and age. They attribute the greater creep in young bone to the lower degree of mineralization and consequently to the relatively greater content of collagen. They also suggest that change in creep properties is a consequence of the rearrangement of mineral and organic components of bone related to diffusion activity and extent of ion exchange between the mineral phase and tissue fluid and the different amount of bound water in bone tissue.

Melnis et al. (1981) conducted longitudinal tensile creep tests to observe the effects of various specimen storage and testing conditions on creep properties of tibial bone. They concluded that wet and dry bone display different creep behavior indicating different deformation mechanisms. The effects of the testing conditions have a greater impact on the behavior of bone as opposed to the storage conditions. Melnis et al. (1982) conducted a statistical study to determine the effects of age, storage/testing conditions, load magnitude, load duration and anatomical location on creep deformation. All factors proved to have a significant effect on the creep behavior and need to be defined for any study.

Carter and Caler (1983) documented the three characteristic stages of creep behavior in longitudinally oriented human bone specimens. They used human femoral cortical bone machined into waisted cylinders and performed creep fracture tests with constant load levels. Specimens were loaded at a constant stress rate of $315 \mathrm{MPa} / \mathrm{s}$ until a pre-determined stress was achieved and then maintained until fracture. Creep fracture behavior of longitudinal oriented bone specimens was similar to other materials where the three characteristic stages of creep are seen. They found the strain at fracture for the constant stress creep-fracture specimen was $0.0179 \pm 0.005$ and was independent of the constant stress level. The influence of hold stress on time to fracture was approximately linear on a log-log plot. Empirical data from log-log plots of peak stress versus time to failure were used to predict the ultimate tensile fatigue strength. The predicted theoretical 
time-dependent curve correlated well with the experimentally determined failure times of tensile fatigue specimens. This suggests that creep damage related to stress magnitude plays a significant role in zero-tension fatigue loading.

Carter and Caler (1985) present a mathematical model similar to a brittle-fracture creep model (Kachanov 1958; 1961) to describe creep-fracture characteristics. The model assumes that the creep process is accompanied by the progressive accumulation of cracks. A damage function, $D_{c}$, is zero when no cracks are present, and 1 indicating creep failure. The application of load results in the accumulation of creep damage that is dependent on both the magnitude and duration of loading. Time to failure, $T_{B}$, is related to stress by a power function:

$$
\mathrm{T}_{\mathrm{B}}=\mathrm{A} \sigma^{-\mathrm{B}}
$$

where A and B are empirically determined constants. Assuming a constant rate of damage accumulation:

$$
\frac{d D_{c}}{d t}=\frac{1}{A \sigma^{-B}}
$$

where $D_{c}$ is cumulative creep damage. If an arbitrary time-varying stress history exists for the specimen, the cumulative creep damage is:

$$
D_{c}(t)={ }_{0}^{t} \frac{1}{A \sigma(t)^{-B}} d t
$$

If the specimen fractures due to the accumulation of creep damage only, the time to fracture, $t_{b}$, is solved by setting $D_{c}\left(t_{b}\right)=1$.

Another damage model is presented to describe the contribution of combined time-dependent and cycle-dependent damage behavior:

$$
D_{s}=D_{c}+D_{F}+D_{I}={ }_{0}^{t} \frac{1}{A \sigma(t)^{-B}} d t+\frac{\omega t}{F \Delta \sigma^{-G}}+0=1
$$

where $D_{S}$ is general stress damage, $D_{F}$ is fatigue damage and the interaction between creep and fatigue, $\mathrm{D}_{\mathrm{I}}$, was assumed to be negligible. For monotonic tensile tests at a constant stress rate, the fatigue contribution was neglected. The model reduced to:

$$
\frac{1}{{ }_{0}^{t_{b}}} d t=1
$$


The relationships between ultimate tensile strength and stress rate and strain rate were derived. The model compared well to other studies with an ultimate tensile strength and a similar strain rate (Crowninshield and Pope, 1974; Burstein et al., 1976; Wright and Hayes, 1976). Experimental work was then conducted to evaluate the model (equation 2.13) for cyclic loading with a non-zero mean stress where creep damage and fatigue damage may accumulate. Failure was due to the contribution of creep dominating damage during the low-cycle region and in the high cycle region fatigue damage dominated. Failure times and number of cycles were described well by the model. The contribution of creep due to cyclic loading appears to be relatively insignificant due to strain magnitudes and number of cycles to failure in bone in-vivo.

Park and Lakes (1986) conducted torsion creep tests at body temperature on prisms of human femoral wet and dry bone. Two load levels were used for the creep tests: $0.05-0.08 \%$ and $0.6-1.2 \%$ initial maximum nominal shear strain. The load tests lasted from 10 seconds to 131 days. Shear slippage at the cement line was observed as the specific mechanism of creep, however, it only accounted for about one-third of macroscopic strain suggesting other deformation processes.

Fondrk et al. (1988) defined a creep threshold by performing creep tests on longitudinally oriented waisted rectangular specimens from human and bovine bone at room temperature. Specimens were loaded in one second, the load was then held constant for 60 seconds, then unloaded in one second, then held at zero-load for 2 minutes. This cycle was repeated with increasing load.

Data from the tests were plotted in stress versus strain plots. At lower loads, the loading cycle was represented by a closed loop where stress is held constant yet the specimen deforms. The bottom of the loop represents recovering deformation where the specimen maintains zero-load. They also found that there was a linear response of the deformation with respect to load function. As the load was increased, the top portion of the loop also increased meaning that during the same holding time, the larger load was causing greater deformation. During the zero-load phase, the loop did not close completely due to unrecoverable, plastic, deformation.

Plots of strain and time were also generated. At lower constant loads, the straintime curves stabilized at an equilibrium strain. At higher loads, however, the specimen 
did not reach equilibrium strain, but steady creep occurred represented by a positive slope.

Two regimes of behavior were observed. Constant lower stresses produced recoverable, elastic deformation that stabilized with time. Higher constant stresses produced unrecoverable, plastic, deformation and a constant creep rate. They proposed a stress level that produces a steady state creep rate of $10 \mu \varepsilon / \mathrm{s}$ as a boundary or threshold to distinguish between these two types of behavior. The steady state creep rate was plotted as a function of stress. The creep threshold represented a sharp boundary where creep rates increased rapidly for stresses above the creep threshold. The relationship was found to be exponential. The creep threshold for human longitudinal bone specimens was 73 $\mathrm{MPa}$ and for bovine bone, $117 \mathrm{MPa}$.

Caler and Carter (1989) measured time to failure for different stress levels for longitudinal bone specimens. They machined human femoral waisted cylinders oriented in the longitudinal direction and tested them in axial compression and tension. A single load cycle was used with a peak stress of $14 \mathrm{MPa}$ at a stress rate of $28 \mathrm{MPa} / \mathrm{s}$. The specimens were loaded to predetermined stress levels until creep failure; compression stress ranged from 108 to $172 \mathrm{MPa}$ and tensile stress ranged from 53 to $120 \mathrm{MPa}$. At the same stress level, the steady state creep rate was higher and the third and final stage of creep was more abrupt in tension rather than compression. Predictions of time to failure based on time-dependent damage compared well to tensile cyclic failure suggesting that tensile cyclic loading creates primarily time-dependent damage. Failure in compressive cyclic loading appeared to not be due to time-dependent damage. Load histories involving both tensile and compressive loading lead to earlier failure than predicted from a simple addition of tensile time-dependent damage and compressive cyclic-dependent damage. The results from the time-dependent damage and cyclic-dependent damage accumulation suggest that there is a complicated interaction between the two types of damage to which in vivo bone is subjected.

Modes of failure and observable differences in the fracture surface appeared to depend on the load, compression or tension, and on the stress level. Specimen failure due to relatively low tensile stress exhibited extensive osteon pull-out and an extremely rough, irregular fracture surface. Tensile specimens tested at higher stresses exhibited 
less osteon pull-out. Compressive specimens had a much different surface. Failure due to low compressive stress occurred in an oblique fracture plane exposing Haversian canals and osteon lamellae. Concentric layers of osteons were seen as steps. At high compressive stress the fracture still occurred in an oblique fracture plane but the osteons were not as exposed, evidence of osteon separation was observed as troughs in the surface. Microstructural features exhibited creep strain over an order of magnitude greater than measured macro creep behavior (Nicolella et al., 1998). Therefore, haversian systems and lacunae may serve as stress risers.

Mauch et al. (1992) used bovine and deer antler longitudinal tensile specimens to determine time to fracture. The bone types were chosen for their large diversity in the elastic modulus values. Predetermined constant loads were chosen that would not cause immediate specimen failure. Application of the load occurred in approximately 6 seconds, which was removed from the total time to fracture. After testing, calcium content was determined and the fracture surface was observed. The fracture surface of the bovine specimens had relatively rough surfaces where the specimen was beginning to fail over a long period of time. The smoother region indicated fast crack propagation. The antler bone, in contrast, had a rough surface regardless of fracture time. The antlers had a lower calcium content than the bovine bone as well as a lower elastic modulus. Small differences in calcium content have been associated with large differences in mechanical properties.

Bone and antler show a reduction in time to failure as $\sigma / \mathrm{E}$ is increased. Antler takes far longer to rupture at any given value of $\sigma / E$ than does bone. Bovine bone begins to suffer damage at a strain of 0.005 , indicated by more compliance upon reloading. Antler undergoes a strain of 0.02 before accumulating significant damage but the stress to attain this strain is lower than for bovine bone.

In contrast to the cumulative damage model of Caler and Carter (1989), strain, not stress, was suggested as the determining factor. A comprehensive model must take into account the markedly different responses of strain of bones of different degrees of mineralization. Less mineralized bone takes much longer to fracture at any given strain, or normalized stress, which seems to not be in accordance with the cumulative damage model. 
Rimnac et al. (1991, 1993, 1993a) conducted tensile creep tests of bovine femoral rectangular waisted specimens at different temperatures. A tensile load was applied for $15 \mathrm{~min}$, if after the $15 \mathrm{~min}$ there was no elongation, then increments of $20 \mathrm{~N}$ were applied for additional $15 \mathrm{~min}$ intervals until elongation occurred, this load was then maintained until failure. The applied loads varied between 71 to $115 \mathrm{MPa}$. After testing, a cross section was taken and the volume fraction of secondary Haversian bone was determined. A model was used to fit the mean of the natural log of the steady state creep rate involving the volume fraction of secondary Haversian bone, stress, and temperature. The experimentally determined creep rate was compared to the predicted creep rate for each specimen. The steady state creep rate of compact bovine bone was significantly affected by the microstructure of the specimen, applied stress, and temperature.

Microstructure was shown to strongly affect the steady state creep rate. Secondary Haversian bone creeps 100 times faster than fully lamellar bone suggesting that remodeling has a negative effect on creep rate. The steady state creep rate was also shown to increase with increasing stress and increasing temperature. They hypothesized that permanent deformation during creep is primarily due to damage mechanisms in hydroxyapatite.

Pattin et al. (1995) used bovine bone waisted cylinders to show temperature and loading mode dependence of creep behavior. Tension and compression creep tests were conducted with an increasing load applied at an "instantaneous" rate, then a constant stress was held for 60 seconds, the load was applied in $10 \mathrm{MPa}$ increments until failure. The instantaneous modulus was determined at each load level from the rapid increase in load. The isochronous modulus was determined from the end of the constant stress increment. Failure stress for both loading modes was less in the high temperature group $\left(37^{\circ} \mathrm{C}\right)$ and the creep rates were also greater. Creep failure was shown to be different between the tensile and compression modes. The instantaneous modulus degraded much less than the isochronous modulus suggesting that an appropriate model of bone as a damaging material should include an instantaneous modulus that degrades much less than the rest of the model. Increasing the temperature decreases strength and moduli, and increases creep rates. The decreasing moduli can be related to microcrack formation by 
an effective loss of load bearing area. Thus, higher temperature and lower strength may be related to increasing time-dependent damage accumulation rates.

Jepsen et al. (1995) tested rate and size effects under monotonic conditions and creep and relaxation of bovine cortical bone waisted cylinders in torsion. Specimens were loaded at a rate of $1.41 \mathrm{Nm} / \mathrm{s}$ and held for 60 seconds at a predetermined torque; 136, $226,249,260,316$, or $362 \mathrm{Nmm}$. The specimens were then released, allowed to recover for 4 minutes, and loaded to failure at $1.41 \mathrm{Nm} / \mathrm{s}$ to measure property degradation. Steady state creep rates increased in a nonlinear manner with increasing load levels.

Vanscoy (1996) loaded cylindrical femoral cortical bone specimens with constant internal pressure. The inner surface of the specimen was machined and the outer surface remained intact. Strain gages were applied to the outer surface and measured tensile and compressive hoop strain. The compressive hoop strains on the surface were attributed to structural properties. The irregular bone geometry caused compressive strain where the thicker cortex acted as a hinge.

Vanscoy et al. (1997) measured radial load to fracture in femoral bone specimens and correlated failure load to cortical bone index measurements from corresponding radiographs of the intact femora. Ten pairs of femurs were wet machined to obtain nineteen proximal cortical bone annular specimens with only their inner cortex machined; the outer surface was left intact. Anterior-posterior (AP) radiographs were taken to obtain cortical index (CI) measurements (Gruen, 1997) at the "standard" level 100 millimeters below the mid-lesser trochanter line and at the levels corresponding to the proximal test specimen locations. An initial internal pressure of 2.95 MPa was applied and then loaded at a mean rate of $0.15 \mathrm{MPa} / \mathrm{s}$ to failure.

The mean ultimate pressure at which the annular specimens fractured was $3.86 \pm$ $1.36 \mathrm{MPa}$ (range $1.84-6.11 \mathrm{MPa}$ ). The average "standard" cortical index was $48.4 \pm$ 11.1 (range 31.3 - 65.5). The average proximal cortical index was $30.2 \pm 7.9$ (range 14.7 41.4). Regression analysis indicated that transverse strength of the femur correlated significantly with $\mathrm{CI}$, with the strength of the correlation increasing when the CI was taken at the proximal test specimen location.

Nicolella et al. (1998) used a multiple loading cycle test regime to conduct a constant stress creep test on bovine bone. The load cycle involved a ramp-up in load until 
a specified global strain level. The stress level was maintained for 2 minutes, released, and zero-load was maintained for 2 minutes. This cycle was maintained at incrementally higher strain levels until failure. Using this cyclic process, a local strain was measured at the beginning of the constant stress phase and compared to the local strain at the end of the load phase as well as to the global strain level. Local creep strains were shown to be over an order of magnitude greater than the measured macro creep.

Bowman et al. (1999) conducted a study with demineralized cortical and trabecular bone to establish the role of collagen in creep behavior. They found that creep behavior of demineralized bone was similar to creep behavior of cortical and trabecular bone. The similarities suggest that collagen was the phase of bone responsible for the creep behavior of both bone types. They also used a composite model to predict accurate power-law regressions for both steady-state creep rate and time to failure as a function of applied normalized stress for cortical, trabecular, and demineralized cortical bone (Bowman et al., 1997).

A damage model was developed by Fondrk et al. (1999) to describe the non-linear behavior of cortical bone. The damage model included two internal state variables to describe the non-linear behavior. Simulations of experimental loading in tension and cantilever bending were conducted using the model with values from previous creep studies (Fondrk et al., 1988, 1999; Fondrk, 1989). Excellent fits of the experimental data were obtained.

Fondrk et al. (1999; 1990) conducted load-unload cycles (0.5 - 0.7 second duration) on tension specimens at high stress rates $( \pm 420 \mathrm{MPa} / \mathrm{s})$ and high stress amplitudes to observe nonlinear strain behavior. For human bone, $29.1 \%$ of the maximum nonlinear strain (total strain - strain due to linear elastic behavior) accumulated after load reversal. The average residual strain recovered during zero-load was $35.4 \%$. Significant volumetric strains were also found supporting damage accumulation corresponding to formation of cracks and voids. Residual volumetric strains suggest only partial closure of induced voids and cracks occurred during unloading. The new void volume and local residual stresses may be a potential stimulus for a biological repair mechanism. Results support the similarity between stress-strain behavior observed during creep loading and transient loading at physiological rates. 


\subsubsection{Time-Dependent Behavior of Transversely Oriented Bone Specimens}

Work mentioned thus far involves bone creep in the longitudinal direction. Human cortical bone is as an orthotropic material (Ashman et al., 1984; Van Buskirk and Ashman, 1981; Knets, 1978; Yoon and Katz, 1976) or has been assumed to be transversely isotropic (Reilly and Burstein, 1975; Huiskes et al., 1981). In either case, the transverse properties (Figure 2.7) are assumed to be different than those in the longitudinal orientation.

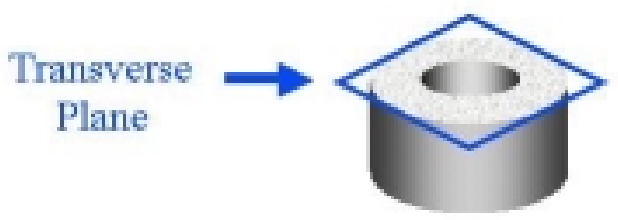

Figure 2.7 The transverse plane is perpendicular to the longitudinal plane.

The time-dependent deformation of bone to loads in the transverse direction (perpendicular to the long axis) has received less attention and has been limited to the study of bovine bone loaded in compression (Utenkin and Sveschnikova, 1973; Tanabe and Kobayashi, 1994), tension (Crowninshield and Pope, 1974), and torsion (Bonfield and $\mathrm{Li}, 1967)$. The results suggest that the time-dependent response of bone differs with bone direction.

Bonfield and Li (1967) loaded bovine bone longitudinal and transverse specimens in torsion at 1 degree/min to observe the viscoelastic behavior. Specimens were loaded and the residual twist was measured after unloading. Small levels of stress, below fracture stress, resulted in non-elastic behavior.

Utenkin and Sveschnikova (1973) tested human compact bone longitudinal and transverse rectangular specimens in compression. The specimens were loaded at a constant stress equal to $50 \%$ and $90 \%$ of the elasticity limits for 90 minutes and then left at zero load for 60 minutes. The resulting creep behavior during the constant stress was dependent on the orientation of the sample, however, the recovery behavior did not depend on specimen orientation. 
Crowninshield and Pope (1974) tested the time-dependent behavior of bovine bone specimens from the longitudinal and transverse direction at a range of strain rates between 0.001 and $200 \mathrm{~s}^{-1}$ in tension. For the transverse specimens tested at a strain rate between 0.001 and $2 \mathrm{~s}^{-1}$, the elastic modulus was consistently $2 \mathrm{GPa}$ compared to an elastic modulus of approximately $11 \mathrm{GPa}$ in the longitudinal direction.

Tanabe and Kobayashi (1994) tested bovine bone cylindrical specimens under impact and quasi-static compressive loading from the longitudinal, radial and circumferential orientations. The goal was to develop a model that includes viscoelastic parameters to represent the non-linear stress-strain characteristics of bone up to a strain rate of $100 \mathrm{~s}^{-1}$. Their model and the results of the mechanical tests compare well. Differences in stiffness in the different directions were noted.

\subsection{Mechanisms of Creep Deformation}

The mechanisms of creep deformation are dependent on the microstructure of a material. In this section the creep mechanisms in metal (Rhoads, 1994), polymers (Lemaitre and Chaboche, 1990), wood (Lemaitre and Chaboche, 1990), and bone are discussed. Creep usually occurs in metals at high temperature. The three stages of creep were discussed (Figure 2.5) in a previous section. During primary creep, strain hardening (dislocation generation and interaction with grain boundaries and other barriers) is occurring at a greater rate than annealing (annihilation of dislocations, climb, cross-slip and some recrystalization). High strains cause necking in a uniaxial tension specimen during the tertiary stage, which further accelerates deformation. Ductile fracture occurs around defects in the solid such as precipitates at high temperature or grain boundaries at low temperatures.

During steady state creep, the rate of strain hardening of dislocations is balanced by the rate of recovery (Dieter, 1986). When the temperature of the material is between 0.3 and 0.7 of its melting temperature, dislocation climb is the primary mechanism of creep (Rhoads, 1994). During higher temperatures, above 0.7 of the melting temperature, creep is analogous to viscous flow. The mechanism of creep is diffusion of atoms from one place to another (Coble or Herring-Nabarro model). 
The mechanism of creep in polymers and wood is different. For the majority of polymers, if the load remains below the elastic limit, deformation is elastic. Elastic deformation results from the relative movement of chain segments in which the bonds are not really destroyed but the rearrangement is thermally activated. Above the elastic limit, irreversible reorientation of chain segments and crystallite wafers occurs resulting in strains, which remain after removing the load. Fracture in polymers initiates with the disappearance of molecular bonds under both external load and thermal activation (Lemaitre and Chaboche, 1990).

Elastic deformation in wood is caused by the reversible deformation of the cells that are arranged in fibers parallel to the axis of the trunk. The principal mechanism of plastic deformation in wood consists of slip of cells over one another. Fracture is caused by the breaking of cell (cellulose) chains, the breaking of bonds by a very high stress in the direction of the fibers, and cleavage fracture at much lower stresses perpendicular to the fibers (Lemaitre and Chaboche, 1990).

It is well known that most collagenous tissue such as bone has a hierarchal structure (Figure 2.8). Complicated mechanical properties should be related to structure. It is important to understand the contribution of each level of the structure to the mechanical properties of collagenous tissue (Sasaki and Odajima, 1996). Many studies examining collagens role in the tensile properties of collagenous tissue have used tendons where collagen fibrils are the principal, tensile-bearing components of connective tissue (Sasaki et al., 1999). Because the same kind of collagen, type I, exists in both tendons and bone (Baron, 1996), studies involving tendons are appropriate to gain insight into collagen within bone. For example, Bowman et al. (1996) showed that in tension demineralized bone behaves much like collagen in soft tissue. 


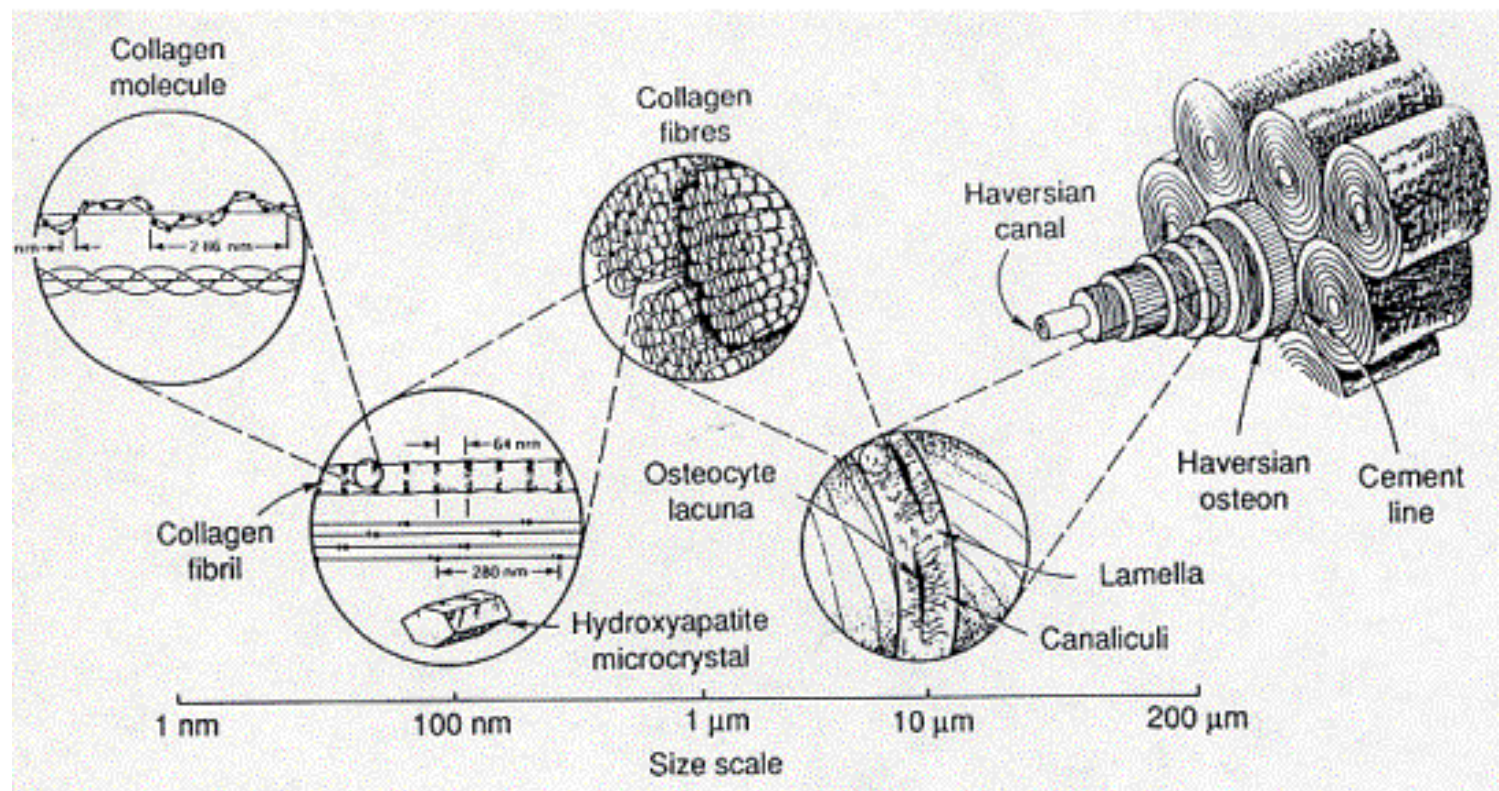

Figure 2.8 The hierarchal structure of bone including osteons, lamella, collagen fibers and collagen molecules (Lakes, 1993).

Before engaging in a discussion about collagens role in bone, collagen and its hierarchal structure must first be defined. Collagen is formed by three polypeptide chains each with an amino-terminal, or N-terminal, at one end, and a carboxyl-terminal, or Cterminal, at the other end of the chain. The following amino acids are present along the chains: glycine (Gly, nearly every third residue, 35\%), proline (Pro), 3-hydroxyproline, 4hydroxyproline, and 5-hydroxylysine (5-Hyl). The last three are posttranslational modifications from normal proline and lysine after the collagen polypeptides are synthesized (Lehninger et al., 1993; Stryer, 1995; Garrett and Grisham, 1999).

The amino acid sequence along the chain is important for specifying the conformation of proteins. The amino acid sequence is generally a repeating tripeptide unit, Gly-x-Pro or Gly-x-Hyp where x can be any amino acid (Lehninger et al., 1993) or repeats of Gly-x-y where $\mathrm{x}$ is Pro and y is Pro or Hyp (Garrett and Grisham, 1999). One strand of the collagen protein is about 1000 amino acid residues in length $(300 \mathrm{~nm})$ and about $1.5 \mathrm{~nm}$ in diameter (Lehninger et al., 1993; Stryer, 1995).

Type I collagen consists of three polypeptide chains, two which are identical and one which is different (Garrett and Grisham, 1999). These strands are hydrogen bonded to each other as they wind together forming a super helical cable amplifying its strength. 
The hydrogen donors are the peptide NH groups of the Gly residues and the hydrogen acceptors are the peptide $\mathrm{CO}$ groups of residues on the other chains. The inner region of the helix is very small allowing only the smallest amino acid residue, Gly, to fit (Stryer, 1995). Gly residues from the three strands stack along the center (Figure 2.9). The NH from Gly on one strand hydrogen bonds to $\mathrm{CO}$ of an adjacent $\mathrm{x}$ residue. The sequence of amino acids allows for the closest possible packing of the polypeptide chains. The tight wrapping provides tensile strength. The strength is enhanced by covalent cross-links between polypeptide chains within the triple helix and between adjacent helices (Lehninger et al., 1993).

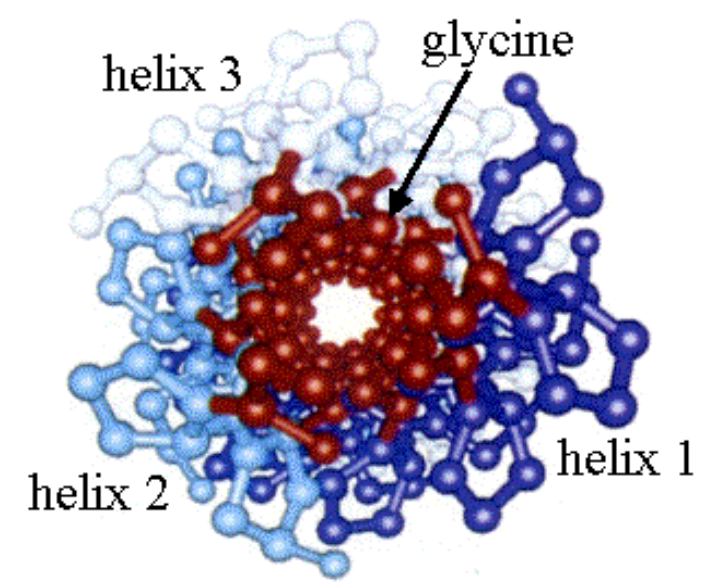

Figure 2.9 The three helices of a tropocollagen molecule wrap tightly around one another. The figure shows one end of the molecule, looking down the center axis with glycine residues stacked along the center (Lehninger et al., 1993, p. 172). 
A three stranded helix of polypeptide chains is called tropocollagen (about $1.5 \mathrm{~nm}$ in diameter) (Figure 2.10). A collagen fibril consists of tropocollagen arranged head to tail (40 nm gaps) in parallel bundles. The heads of adjacent molecules are staggered laterally, each about one-fourth its length, and five across a fibril. The concept of quarter staggering, combining overlap and gaps between tropocollagen, was suggested as a result of the light and dark bars in an electron micrograph. The alignment of the head groups of very fourth molecule produces characteristic cross striations $64-68 \mathrm{~nm}$ apart. The distance between the dark bars seen in the micrograph is called the D period and may be used to measure deformation of the collagen.
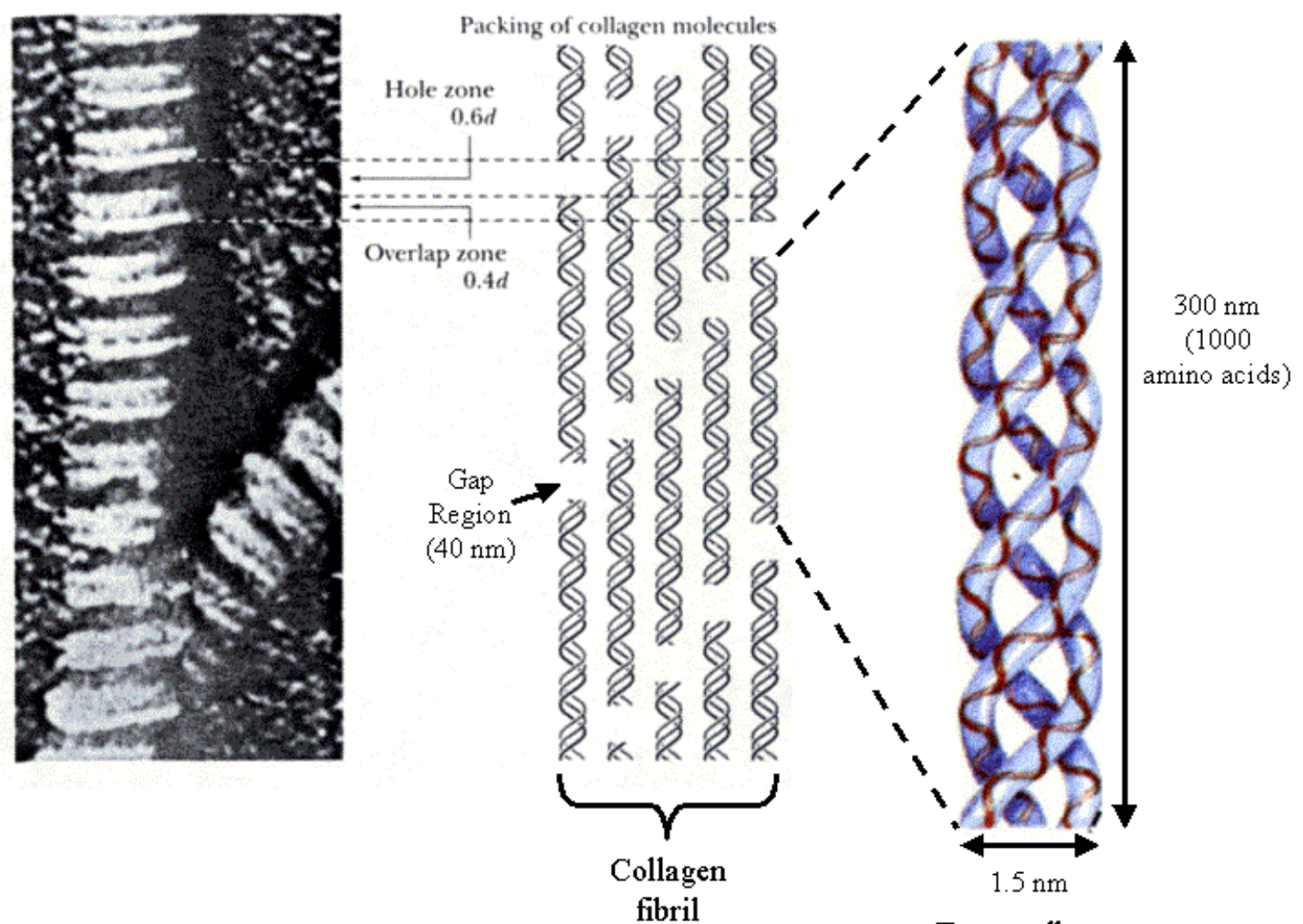

Tropocollagen

Figure 2.10 The tropocollagen is made of three helices. The collagen fibril has tropocollagen staggered five rows across. An electron micrograph shows the striated appearance due to the gap regions (Garrett and Grisham, 1999, p. 177; Lehninger et al., 1993, p. 174). 
The gap regions are important for at least two reasons. First, sugars are found covalently linked to 5-Hyl. The carbohydrates presence suggests it may play a role in organizing the fibril assembly. Second, the gap regions may play a role in bone formation. When bone forms, hydroxyapatite crystals occur at intervals of $68 \mathrm{~nm}$. The space between tropocollagen may be the sites of nucleation for the mineralization of bone.

Fibrils are strengthened and stabilized by both intra-tropocollagen and intertropocollagen cross-links. Intra-collagen cross-links are formed between lysine residues on the non-helical N-terminal region of the tropocollagen (Figure 2.11). Another intratropocollagen cross-link is the hydrogen bond between glycine and an adjacent amino acid residue. Inter-chain hydrogen bonds involving hydroxyproline also stabilize and strengthen the tropocollagen (Garrett and Grisham, 1999).

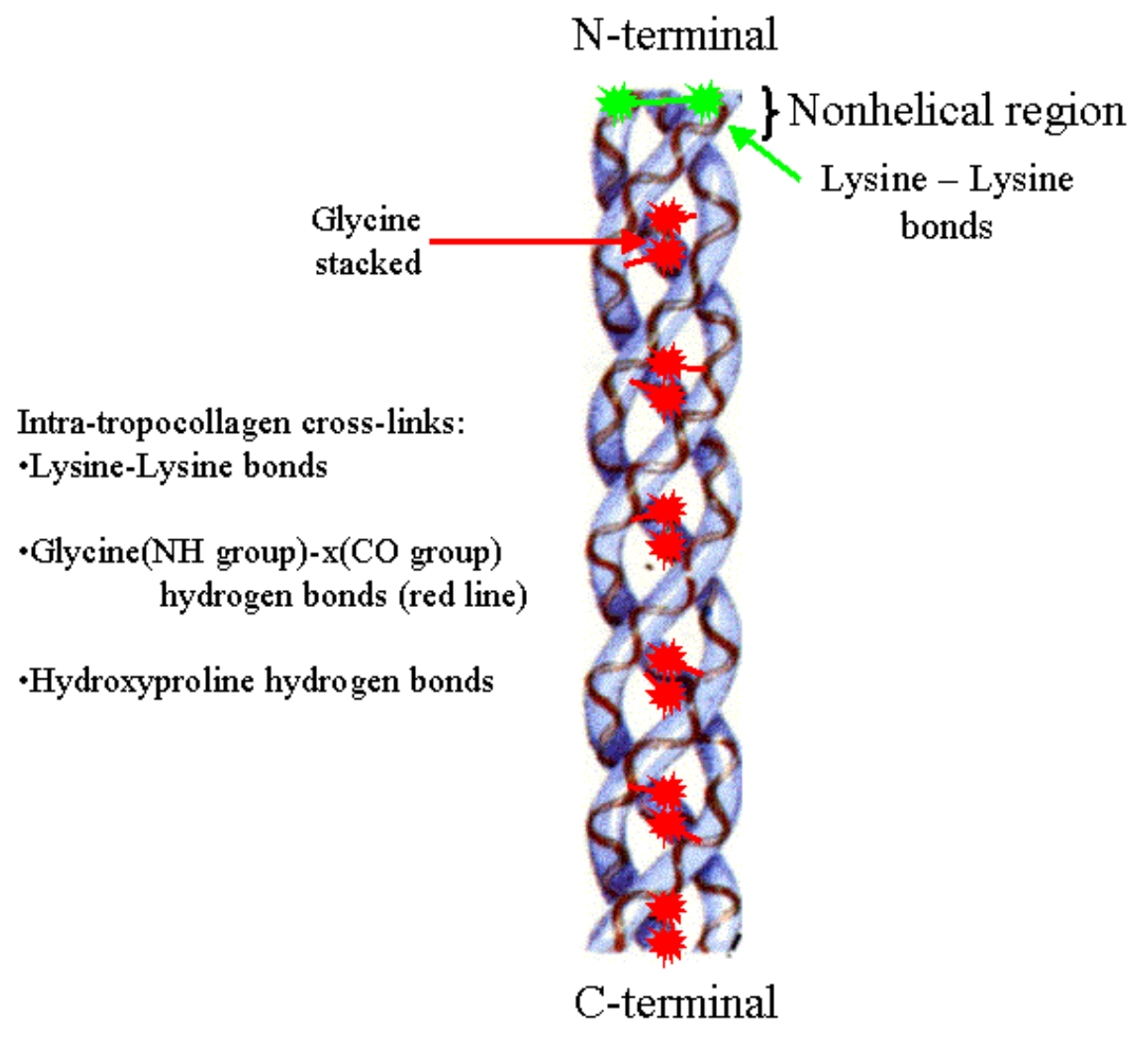

Figure 2.11 Intra-tropocollagen cross-links: 1. Cross-links between lysine residues at the nonhelical N-terminal region; 2. Glycine hydrogen bonds to other amino acid residues; 3. Hydroxyproline hydrogen bonds between strands. 
The inter-tropocollagen cross-linking involves a hydroxypyridinium structure composed of one lysine and two Hyl residues at both the N-terminal region of one tropocollagen and the $\mathrm{C}$-terminal region of an adjacent tropocollagen in the fibril (Figure 2.12) (Garrett and Grisham, 1999) Reductions were reported in bending stiffness, strength, deflections before failure in rat bones when the number of hydroxypyridinium cross-links are reduced (Zioupos et al., 1999).
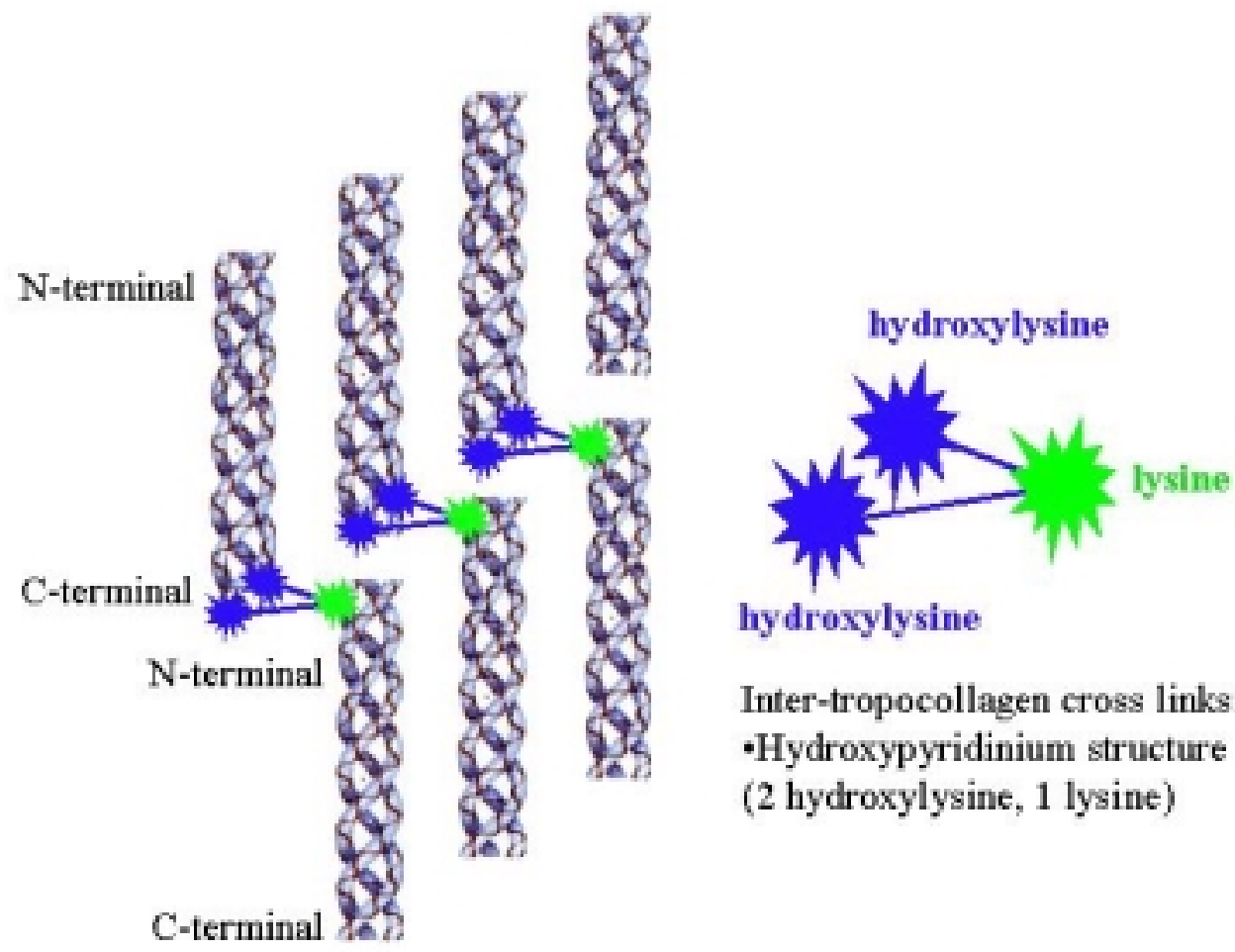
hydroxylysine

Inter-tropocollagen cross links: -Hydroxypyridinium structure (2 hydroxylysine, 1 lysine)

Figure 2.12 Inter-tropocollagen cross-links: Hydroxypyridinium structure composed of two hydroxylysines at the C-terminal and one lysine amino acid residue at the $\mathrm{N}$ terminal. 
Deformation behavior due to tensile load in the longitudinal direction of the collagen orientation has been studied in tendons. Sasaki and Odajima (1996) proposed three deformation modes for collagen (Figure 2.13). When a tendon fiber is elongated along its fiber axis, the helix of the collagen molecule has been shown to also elongate (Cowan et al., 1955; Sasaki and Odajima, 1995). Therefore, molecular or tropocollagen elongation is a possible mechanism (mode 1). Two types of molecular rearrangements are possible. One, the gap region between longitudinally oriented molecules increases (mode 2). Second, the relative slipping of laterally adjoining molecules along the fiber axis (mode 3). They consider that any deformation of collagenous tissue occurs as a result of a combination of all three modes of deformation.

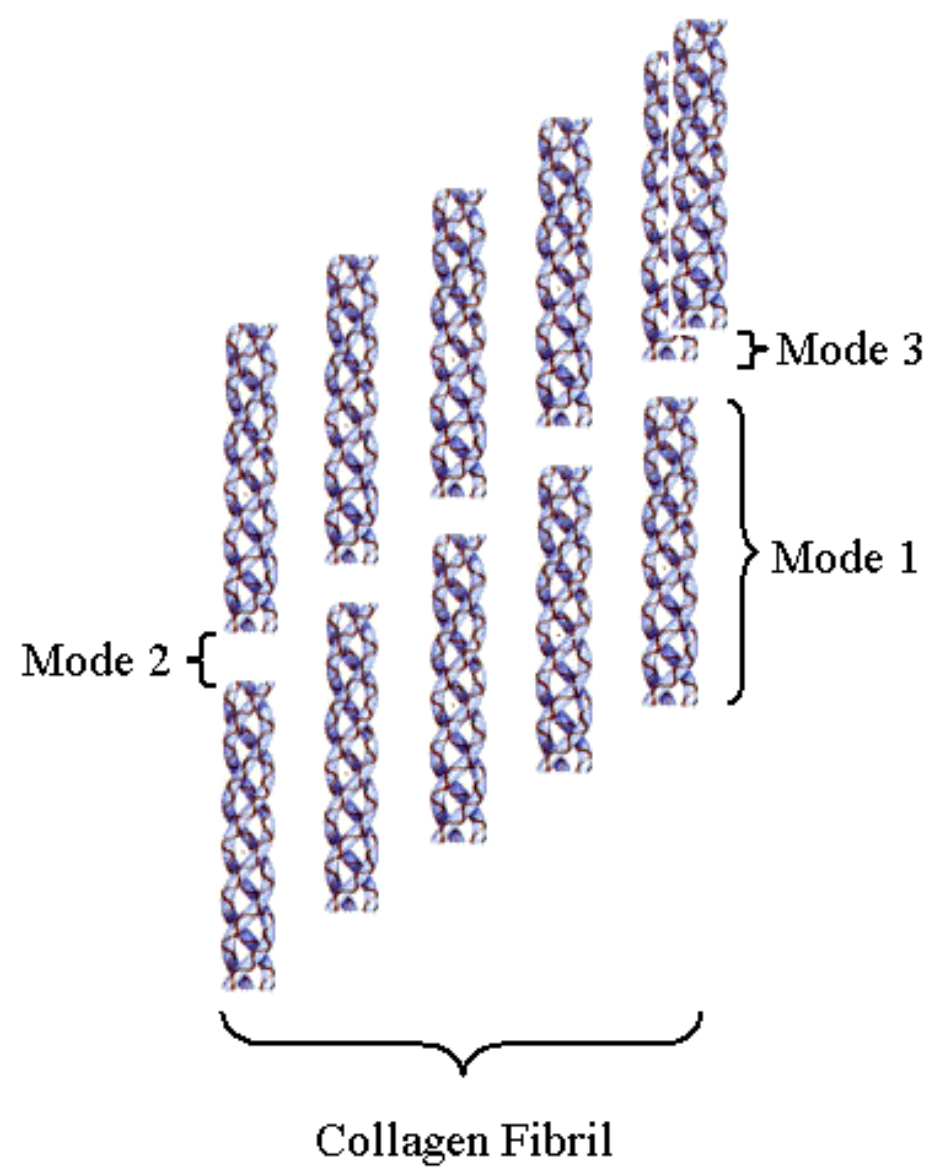

Figure 2.13 The three modes of deformation within a fibril as defined by Sasaki and Odajima (1996). Mode 1 is tropocollagen elongation, mode 2 is elongation of the gap region, and mode 3 is slippage between laterally adjoining tropocollagen molecules. 
Each rearrangement mode (modes 2 and 3) when occurring alone, causes a tilt in the pattern against the fiber axis. Short angle X-ray diffraction (SAXD) pattern has shown that collagen under tensile stress does not undergo a change in the tilt pattern (Nemetschek et al., 1979). Therefore modes 2 and 3 must occur simultaneously (Sasaki and Odajima, 1996).

If the D period (distance between dark regions in the SAXD pattern) elongates $\left(\varepsilon_{\mathrm{D}}\right) 2 \%$, then tropocollagen elongation (mode 1) would account for $1.7 \%$ while modes 2 and 3 would account for $0.3 \%$ of the elongation. Therefore, the major contribution to deformation of the fibril is due to tropocollagen elongation (Sasaki and Odajima, 1996).

In a similar study (Sasaki et al., 1999) for stress up to $8 \mathrm{MPa}, \varepsilon_{\mathrm{D}}$ occurred due to tropocollagen elongation. However, in stress over $10 \mathrm{MPa}$ creep deformation occurred as a result of elongation and molecular rearrangement, modes 2 and 3. This suggests different deformation mechanisms are involved at different load levels. Purslow et al. (1998) found no changes in D spacing or angular orientation of collagen due to creep at 0.01-0.05 $\mathrm{MPa}$ indicating that collagen fiber reorientation may not be the primary source of viscoelastic deformation at these load levels in connective tissue. Relaxation processes in the matrix surrounding the collagen fibers and relaxations within the fibers themselves are possible mechanisms. At higher load levels, near the tendon breaking point, Mosler et al. (1985) observed changes in the $67 \mathrm{~nm}$ D-period spacing in rat tail tendon and human digital flexor tendon under axial loading. The force may be transferred from overlapping units by the matrix which allows the molecular aggregates of collagen to slide with respect to each other.

Davidson (1989) speculates that creep is a manifestation of tropocollagens in a fibril, slipping and reestablishing new cross links. Cohen et al. (1976) suggests that the creep process in a tendon could be composed of two mechanisms: inter-fibrillar sliding at low stresses and fibrillar creep at high stresses. This theory appears to contrast Sasaki et al. (1999) where inter-fibrillar sliding does not occur until high stresses and elongation at low stresses.

Suzuki et al. (1980) suggest that water bridges help stabilize collagen molecules by forming inter-chain (intra-tropocollagen) water bridges of hydrogen bonds (Figure 2.14) of carboxyl groups not engaged in direct inter-chain bonding and at the same time 
permit the inclusion of an additional water molecule linking the bridge with hydroxyprolyl residues. In a fully hydrated state, the bridging water molecule will be frequently exchanged with other water molecules (Sasaki and Odajima, 1996). The exchange means the breaking and formation of bonds, an energy dissipating process. Therefore, they suggest that viscoelasticity of collagen (Fung, 1968) may be related to the hydrogen bond network in the collagen molecule. Glycine, a nonpolar, hydrophobic amino acid is stacked tightly along the inner axis of the triple helix. This hydrophobic region may repel water molecules contributing to instability and assisting in the degradation and formation of new bonds. Melnis et al. (1981) suggest that polar water molecules reduce the cross-linking effect and electrostatic interaction. Thus, a decrease in the amount of fluid (drying or compression) may increase the strength of cross-links and thus strengthen collagen. Water molecules moving in and out of the tropocollagen molecule, reducing the strength of cross-links might allow for slippage and reestablishment of cross-links.

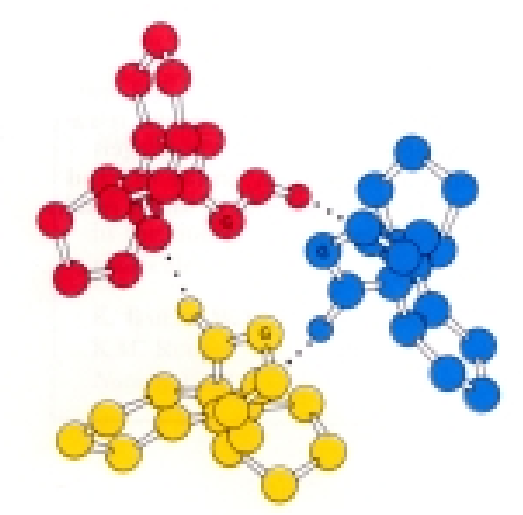

Figure 2.14 The hydrogen bonds between the three stands of a tropocollagen molecule, thin cross-section view. The hydrogen bonds are represented by dots and $\mathrm{G}$ is the alpha carbon of the glycine amino acid residue. (Stryer, 1995, p. 32).

Several creep mechanisms have been proposed for cortical bone. An excellent summary can be found in Bowman et al. (1999). On the ultra-structural level possible creep mechanisms include dislocation generation or cracking of hydroxyapatite mineral crystals, shear between collagen fibrils and subsequent failure of the collagen cross-links, or separation (debonding) of the hydroxyapatite crystals and the collagen matrix (Melnis et al., 1981; Fondrk et al., 1988; Rimnac et al., 1991, 1993, 1993a; Mauch et al., 1992). 
On the micro-structural level possible creep mechanisms include cumulative damage within individual lamella, damage between lamellae within osteons, or damage between osteons and bone matrix resulting in cement line slippage (Lakes and Saha, 1979; Carter and Caler, 1983; Park and Lakes, 1986; Caler and Carter, 1989; Rimnac et al., 1991, 1993, 1993a). Carter and Caler (1989) observed extensive osteon pullout in human bone and lamella separation from one another as a result of tensile creep fracture from low stress levels. Cement line failure was seen at high stress levels. Park and Lakes (1986) found that approximately one third of the creep strain was due to cement line slippage in their study of human bone loaded in torsion hinting at a combination of mechanisms.

Demineralized bone (hydroxyapatite removed) was shown to have similar creep behavior to trabecular and cortical bone suggesting that collagen is the phase responsible for creep behavior of cortical and trabecular bone (Bowman et al., 1996, 1999). The exact creep mechanism however was not identified.

It has been suggested that permanent creep strain in bovine bone is primarily due to dislocation genesis and dislocation interactions in the bone mineral, hydroxyapatite, at the ultra-structural level (Rimnac et al., 1993). However, the crystalline structure of hydroxyapatite creeps only at temperatures much higher than body temperature which suggests that creep in the mineral phase of bone is not likely a mechanism of bone creep behavior (Bowman et al., 1999).

Mechanisms for hoop creep behavior have not been addressed. Due to the difference in microstructure of cortical bone in the longitudinal and hoop direction, creep mechanisms are most likely different.

Collagen fibers in tendons are aligned in the direction of the longitudinal axis of the tendon. In cortical bone, collagen fibers are layered in the lamella of osteons that run in the primary load direction of the bone (Figures 1.4 and 1.5). Frasca et al. (1977) using scanning electron microscopy (SEM) and x-ray diffraction observed single osteons and found different orientations of collagen fibrils. X-ray diffraction patterns revealed "dark", "bright" and "intermediate" patterns under crossed Polaroids. Inspecting these osteons with SEM revealed a variation of collagen fibril orientations. "Dark" osteons contained almost exclusively longitudinally oriented fibrils, and in "bright" osteons, longitudinal 
and transverse fibrils occurred. "Intermediate" osteons were dominated by longitudinal and nearly longitudinal fibrils, but coexisted with oblique fibrils of diagonally opposite orientation.

The hoop creep behavior is most likely the result of a combination of deformation modes as was seen in tendons. Some elongation of the tropocollagen molecules may occur (mode 1, Figure 2.13) and perhaps some rearrangement of cross-links (modes 2 and 3). The fibrils oriented in the hoop direction of specific lamella may deform as was described for tendons since the load direction would be approximately aligned in the direction of the transverse fibrils (Figure 2.15). Fibrils oriented in the longitudinal direction of specific lamella may not deform since a hoop stress is perpendicular to its axis.

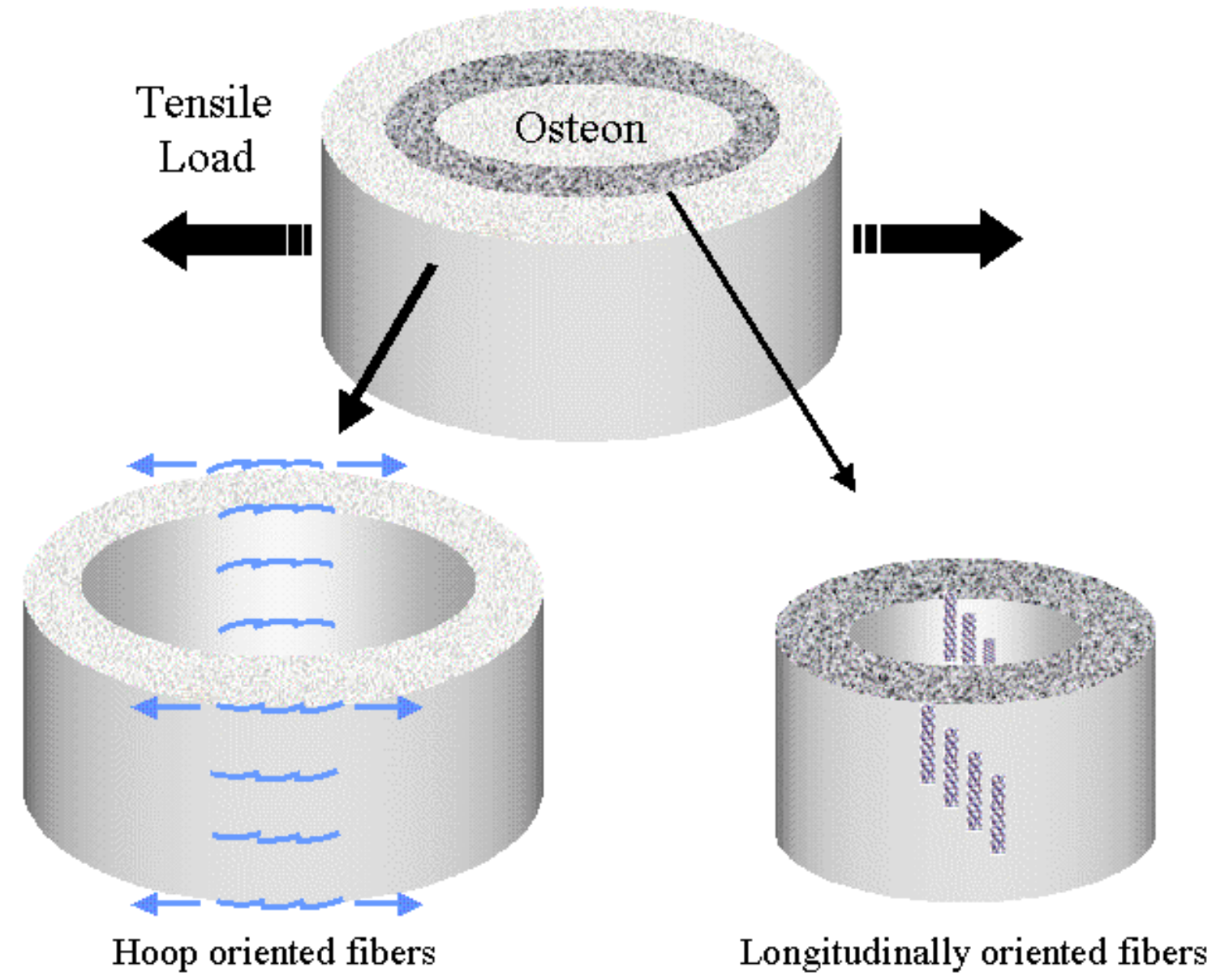

Figure 2.15 Fibers oriented in the hoop direction may deform as suggested for tendons, however, fibers oriented in the longitudinal direction may not deform. 
Fibrils oriented in oblique directions may deform in a variety of ways under tensile hoop stress (Figure 2.16). One mechanism (case 1) may involve a change in the angle of the helices with respect to one another, essentially a pivoting mechanism. The gap regions and terminal bonds remain in their original conformation. A second mechanism (case 2) involves the expansion of the gap regions while the terminal bonds and tropocollagen axis remains in their original conformations. A third mechanism (case 3) involves the expansion of the gap regions causing the terminal bonds to rotate. In this case the tropocollagen axes would remain in their original conformation.
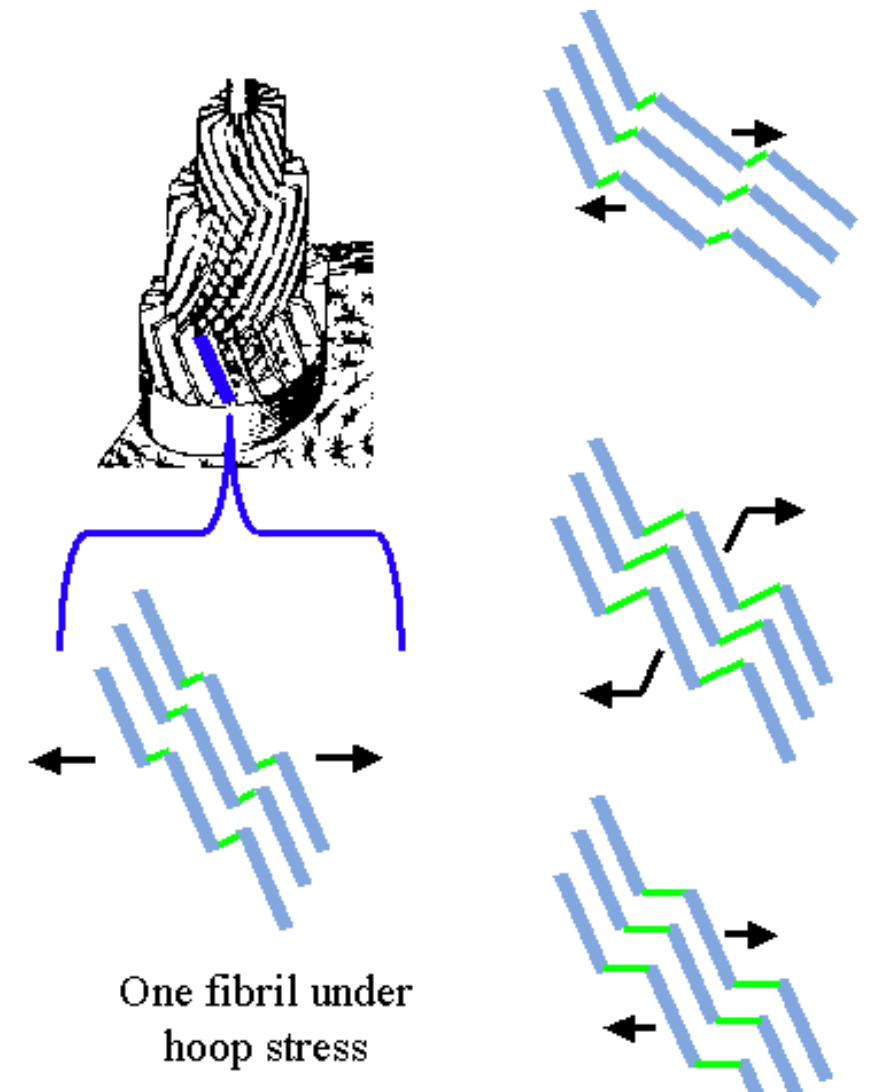

Case 1

Gap regions remain same

Terminal bonds remain same

Helix axes rotates

Case 2

Gap regions expand

Terminal bonds remain same

Helix axes remain same

\section{Case 3}

Gap regions expand

Terminal bonds rotate

Helix axes remain same

Figure 2.16 Deformation mechanisms of collagen fibril under hoop stress. 
A fourth mechanism involves the slipping of tropocollagen within a fibril (Figure 2.17). Bonfield and $\mathrm{Li}$ (1967) suggested that the difference in behavior between longitudinal and transverse torsion specimens was a result of the fibers, or groups of fibers oriented at angles less than $90^{\circ}$ to the longitudinal axis. Most likely hoop creep behavior is a result of several mechanisms.

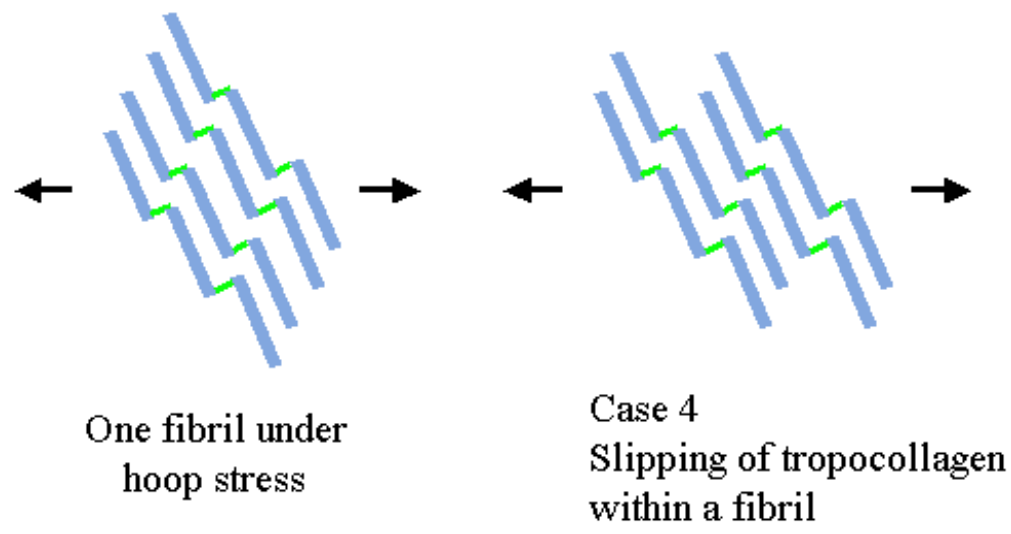

Figure 2.17 Slipping of tropocollagens with an obliquely oriented collagen fiber subjected to tensile stress.

Another mechanism for hoop creep deformation is the generation of cracks on the ultra or microstructural level. The damage mechanisms on the ultra-structural level may be similar to the damage proposed for longitudinal deformation discussed previously. A crack model developed by Fondrk et al. (1997) suggests that damage accumulation due to tensile loading occurs on the fibril or sub-fibril scale. On the microstructural level, hoop creep mechanisms may include cumulative damage within individual lamella and damage between osteons and bone matrix. 


\section{CHAPTER 3}

\section{SPECIMEN MACHINING AND TESTING EQUIPMENT}

\subsection{Specimen Selection and Bone Availability}

Cadaveric material was obtained from the West Virginia University Human Gift Registry in the Department of Anatomy as well as from the International Institute for the Advancement of Medicine (IIAM). A few restrictions were placed on potential donors. Those with chronic illness, such as cancer or diabetes and long-term immobilization were rejected. Potential donors who had received approved antiresorptive agents (estrogen, calcium, and calcitonin) to decrease the rate of bone loss were not excluded. Only femurs from the right side of the cadaver were used. Historically, obtaining cadavers at West Virginia University has not been difficult, especially for the older ages.

\subsection{Specimen Preparation}

The bones were frozen in a $-15^{\circ}$ freezer in storage bags. During all cleaning, cutting, machining and testing, the bone was irrigated with saline solution. The soft tissue surrounding the cortical shell was carefully removed with a scalpel and the bone marrow was removed from the intramedullary canal of the bone. Once cleaned, radiographs were taken facing the anterior side of the bone (AP view) (Figure 3.1). A second radiograph was taken facing the medial side (ML view). Some femurs did not have the lower epicondyles intact, therefore, the distal end of the femur was placed in a Plexiglas frame that contains two metal screws to tighten the frame onto the bone. On two sides of this frame were metal washers that were perpendicular to each other (one per side). These washers serve as a reference to see the effects of magnification on the radiograph. The frame elevates the distal femoral shaft approximately one inch off the table to emulate the bone's position if the lower epicondyles were still attached. The frame also makes it easy to position the femur such that the lateral view was exactly perpendicular to the AP view. For all $\mathrm{x}$-ray examinations, the $\mathrm{x}$-ray machine was set to exactly the same settings (65 kilovolts, 1/60 second, 100 milliamperes) and each femur was positioned in the same manner on the table. 


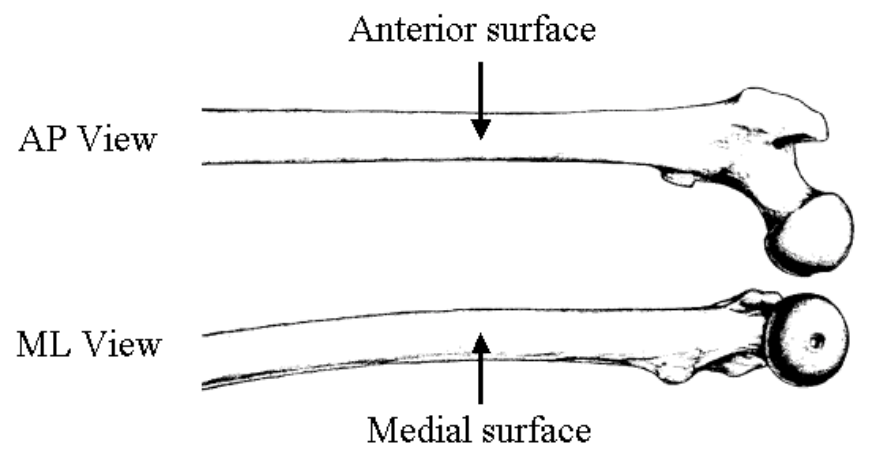

Figure 3.1 $\mathrm{AP}$ and $\mathrm{ML}$ views of the right femur. The two $\mathrm{x}$-rays were taken perpendicular to one another.

The specimens were extracted from the mid-diaphysis of the femoral shaft. This region of the femur consistently had the thickest cortex. Because of the machining protocol, a thick cortex was needed to obtain the cylindrical shape of the specimen. The portion of the femur proximal to the $95 \mathrm{~mm}$ mark was used in previous research. The following details for machining the bone specimens are also found in Vanscoy (1996).

The length of bone was placed into a slotted metal holding apparatus known as the U-clamp device. The bone was placed in the fixture so that its longitudinal axis coincided with the longitudinal axis of the fixture. The $\mathrm{U}$ shaped clamps were placed over the bone and through the metal block and secured with helical spring lock washers (Figure 3.2). Extreme care was taken not to tighten the clamps to the bone too tightly because splitting fractures in the bone would occur.

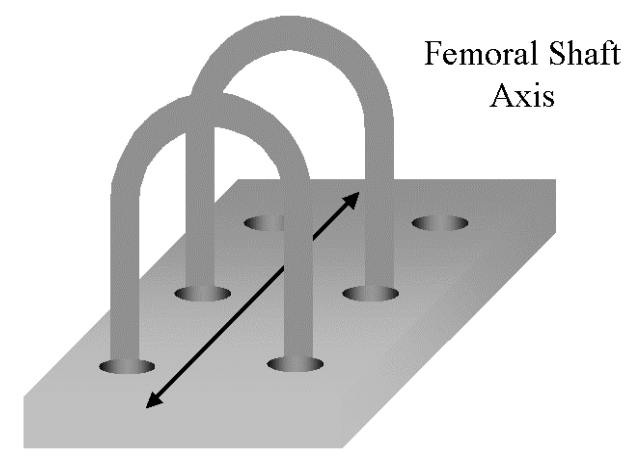

Figure 3.2 U-clamp device for holding the femoral shaft for making the transverse cut.

The fixture device was placed in a vise located on a horizontal mill. The bone specimen was aligned to be horizontal with the milling arm of the mill. Two $127 \mathrm{~mm}$ (5 
inch) diameter circular blades, with a thickness of $1.588 \mathrm{~mm}$ (1/16 inch), are positioned on the cutting arm such that the distance between them was $19 \mathrm{~mm}$ ( $0.75 \mathrm{inch})$. This was the length of the final specimens (Figure 3.3). This procedure was important to parallel ends of the bone specimen. During all machining processes, the bone specimen was kept wet by manually applying saline solution. To protect the machinist, this step of the cutting procedure was done under a vacuum hood to remove any flying debris such as bone dust and chips from the work area. After the ends were paralleled, the test specimen was cleaned in a saline bath and visually inspected for damage. The quadrants were marked on the bone: posterior, anterior, medial, lateral, and the proximal and distal ends. A jeweler's saw and file were used to trim the edges.

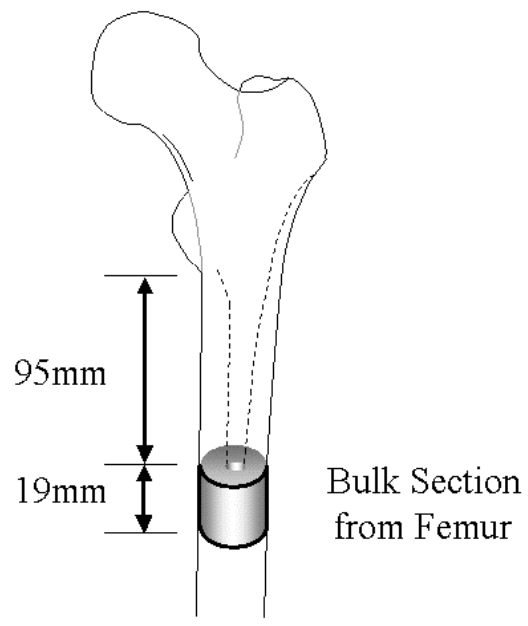

Figure 3.3 A bulk section, $19 \mathrm{~mm}$ in length, was removed from the femoral shaft approximately $95 \mathrm{~mm}$ distal to the lesser trochanter.

\subsection{Inner Cortex Reaming}

Next the inner cortex of the bone specimen was machined. This procedure reamed the inner cortex of the bone specimen to remove trabecular bone. The bone specimen was aligned so that its longitudinal axis was aligned with the vertical axis of the mill. While in this fixture the bone was adjusted to ensure that the desired cortical bone was left behind. Cutting off-center was not a problem and was useful in leaving as much cortical bone as possible. A milling plan was developed on both of the specimen ends to make a cut leaving as much cortical bone as possible (Figure 3.4). Due to the tapered edges of the specimen, the aligning adjustment was needed to account for both ends of the specimen. 
The specimen was placed into the bone-reaming fixture where the sides of the bone were constrained with setscrews. Once the specimen was aligned in the fixture, it was placed in the vertical mill. A $76.2 \mathrm{~mm}$ (3 inch) length, $19 \mathrm{~mm}(0.75 \mathrm{inch})$ diameter, four fluted endmill was used. The bone-reaming fixture was locked into place using a vise on the mill's movable tabletop. Small adjustments were made according to the end mill's projected cut. If the edges of the end mill were not close to the milling plan mapped on the specimen, small adjustments could be made to correct the cutting, less than $1.58 \mathrm{~mm}$ (0.062 inches) in any direction.

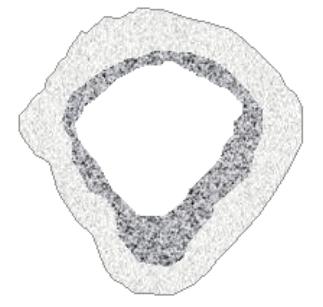

Original Specimen

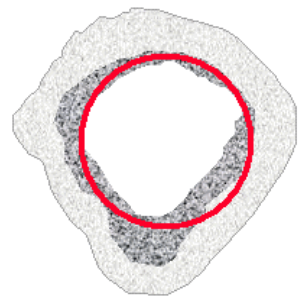

$19 \mathrm{~mm}$ Projected Cut

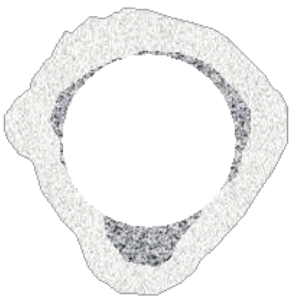

Inner Cortex Cut

Figure 3.4 Milling plan for removal of inner cortex of the bone.

Using a slow cutting speed, $170 \mathrm{rpm}$, and an automatic feed rate of 0.0381 $\mathrm{mm} / \mathrm{rev}(0.0015 \mathrm{in} / \mathrm{rev})$, the reaming commenced through the entire length of the specimen (Figure 3.5). The slow cutting rate prevented the bone from becoming burned due to friction. During this machining the specimen was wet with saline solution.

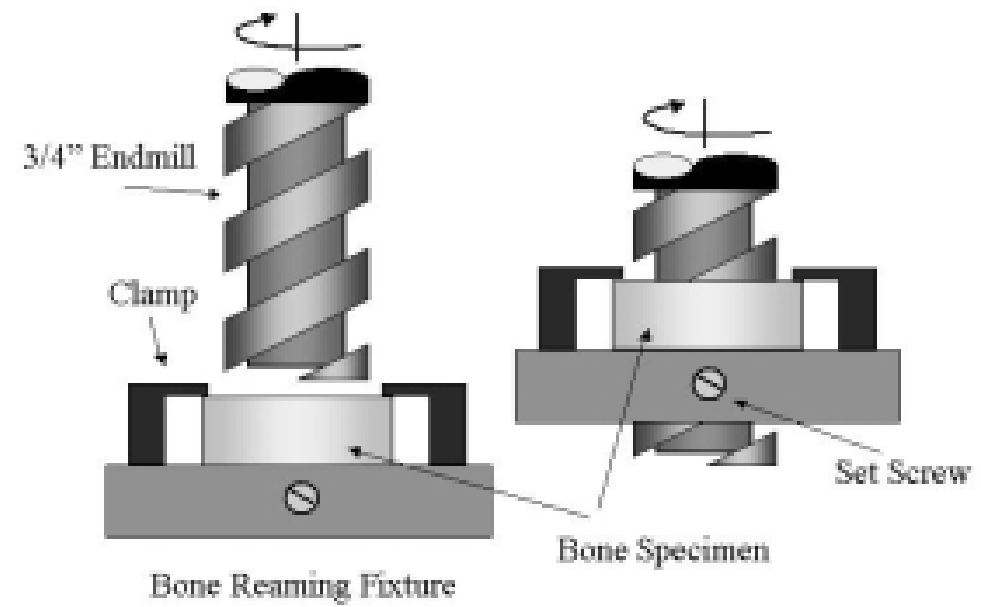

Figure 3.5 Bone reaming fixture for removal of the inner cortex of the bone. 


\subsection{Outer Cortex Reaming}

After the inner cortex was machined, the outer cortex was machined. In order to machine the outer cortex a method of griping the bone for machining was necessary. An expanding mandrel was machined from an aluminum rod, $119 \mathrm{~mm}$ (4.19 inch) in length, $19 \mathrm{~mm}$ (0.75 inch) diameter (Figure 3.6). A diamond saw (Buehler, Ltd., Lake Bluff, IL) was used to cut one thin slot in the rod approximately $29 \mathrm{~mm}$ (1.125 inch) long on one end through the rod. Another notch was cut perpendicular to the first cut. The middle of the rod, where the slots intersected, was then drilled and tapped for a setscrew to be placed into the hole. A screw was then inserted and turned until the screw caused the sides of the rod to migrate outwards, fitting to the insides of the bone. Caution was needed so that the bone did not fracture by turning the screw too far and applying too much internal pressure.

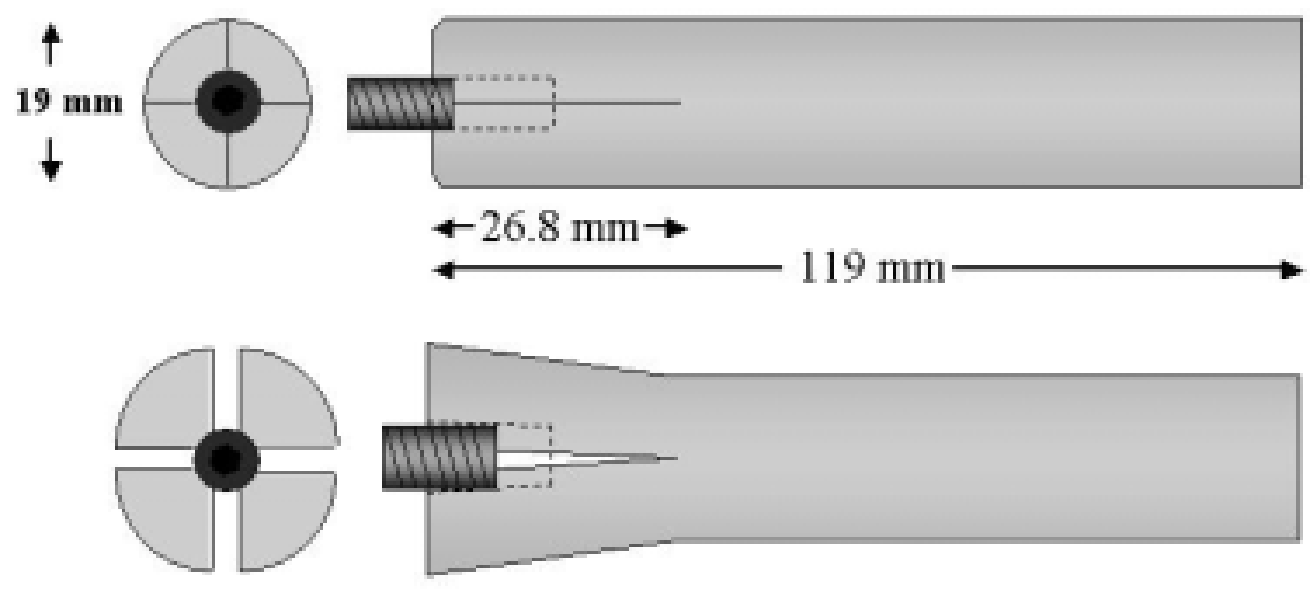

Figure 3.6 The expanding mandrel.

Once the bone was snugly fit on the mandrel to prevent slippage, the end of the mandrel was placed into a lathe. The milling path was predicted before cutting, the thickness of the cylinder was estimated by observing the thinnest cortex of the cross section (Figure 3.7). The sides of the bone, anterior, posterior, medial and lateral, were marked on the cross section of the bone along the inner cortex so that the sides of the bone were known once the outer cortex is machined. During this process surface irregularities were removed which prevented standardization of test specimens. 


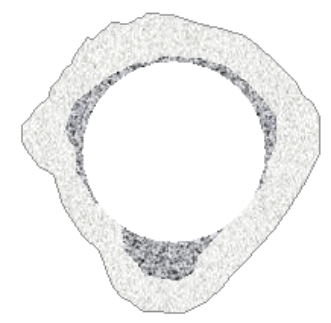

Specimen with Inner Cortex Cut

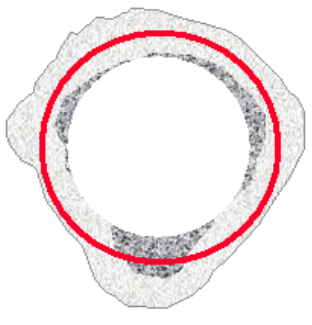

Milling Plan

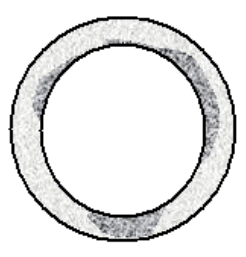

Finished

Specimen

Figure 3.7 The milling path for the outer cortex of the bone.

The bone was turned until the entire surface was circularly uniform. The center of the specimen was marked with a pencil as the bone rotated on the lathe, this pencil mark serves as the guideline for strain gage placement. After the machining process was complete, the electrical resistance strain gages could be placed on the bone.

\subsection{Electrical Resistance Strain Gage Application}

Directly measuring bone surface strains has become a standard technique in the study of bone mechanics. The application of electrical resistance strain gages is commonly used in a variety of biomechanical studies for either in-vivo or in-vitro experiments (Bessman et al., 1982; Crowninshield et al., 1980; Crowninshield and Tolbert, 1983; Rohlmann et al., 1982; Oh and Harris, 1978; Hustosky et al., 1996).

Strain gages (EA-06-125AD-120, Measurements Group, Inc., Raleigh, NC, USA) were used for measuring hoop strain at equal intervals around the circumference of the cylinder. One gage was placed at each of the cortex quadrants: anterior, posterior, medial, and lateral regions (Figure 3.8). The gages were attached around the circumference of the cylinder along the mid-line. 


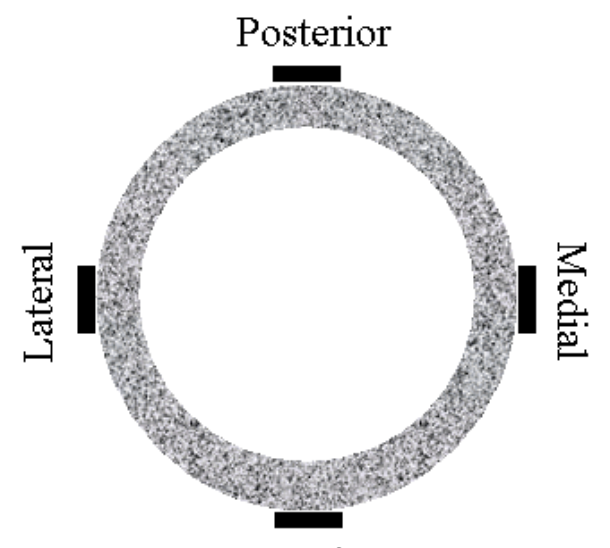

Anterior

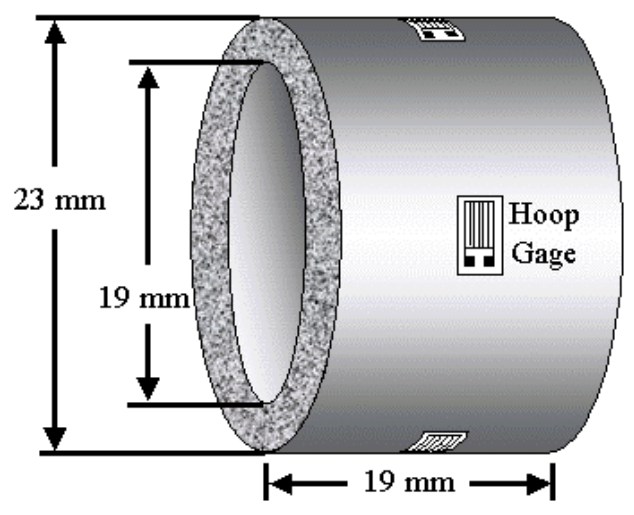

Figure 3.8 The cross section of bone with strain gages at each quadrant and along the mid-line of the specimen.

During application of the strain gages, the surface of the bone specimen directly under the gage must remain dry. The specimen's thickness is thin enough so that keeping the outer surface dry means the entire specimen remains dry. Currey (1988) showed the process of drying and re-wetting has a minimal effect on certain mechanical properties. Although drying the bone is not desired, it is a necessary step to apply the strain gages properly.

The strain gages were each examined under a high-powered microscope to inspect for a faulty bone-gage interface. The resistance of each gage was checked with an ohmmeter. Lead wires were soldered to each gage. Each wire was checked for proper resistance with an ohmmeter to verify that the solder was well applied. M-Bond (Measurements Group, Inc., Raleigh, NC, USA) adhesive was used around the base of the lead wires to assist in stress-relief to protect the wire-tab connection. After an exhaustive selection and testing process to determine the best waterproofing agent, SealAll (Electric Producers, Inc.) proved to be the best at protecting the strain gages and was elastic to not constrain the bone during testing. The waterproofing was needed to protect the strain gages and the exposed wire ends from the corrosive saline preventing short circuits. Once the waterproofing had cured, the specimen was left in the heated saline bath overnight to insure full hydration. 


\subsection{Measuring Strain from the Gages}

The raw voltage collected by the computer data acquisition system (DAS) and converted to microstrain using the following equation:

$$
\varepsilon=\Delta v \frac{(r+1)^{2}}{(\text { Gain })(\text { GageFactor })(\text { Excitation })}
$$

When the circuit was initially balanced, $\mathrm{r}$ equals 1 . The gage factor was taken from the strain gages, the gain was from the conditioning boards where the excitation was 1 volt and the DC coupling filter was and must remain at 10. For the short-term bone specimen testing, $\mu \varepsilon=386.47 \Delta v$ where the gain was 5000 and the gage factor was 2.07.

For the short-term tests, data was collected using a data collection program in LabTech Notebook (Laboratory Technologies Corporation, Wilmington, MA) (program name "cb1"). The sample frequency was $20 \mathrm{~Hz}$ and the strain measurements had a resolution of $1.8871 \mu \varepsilon($ gain $=5000$, excitation $=1$, strain gage factor $=2.07)$ and the pressure had a resolution of $6.68 \mathrm{~Pa}(0.97 \mathrm{psi})$. The gain was chosen to provide the smallest value for resolution yet allow for a scale large enough to capture the range of strain data expected $(386.47 \times 10$ volts $=3864.7 \mu \varepsilon$ maximum $)$.

\subsection{The Testing Fixture}

To provide a more realistic assessment of bone behavior, a technique was established by Vanscoy (1996) and modified to apply internal pressure to simulate the radial forces after the insertion of a maximum fit and fill press-fit implant. The testing apparatus consisted of a device that applied outward radial load to cylindrical tubes of cortical bone. A device was developed that utilizes nitrogen gas to control loading rate and hold stress by applying internal pressure to a hollow right cylindrical specimen (Figure 3.9 and 3.10).

The design of the device used in this investigation was based on equipment used to conduct a hydraulic ring-expansion test (ASTM 1990). It applies radial stress to a cylindrical, non-biological, specimen. According to the standard, a rubber gasket of appropriate diameter is placed within the $76.2 \mathrm{~mm}$ (3 inch) long specimen. Oil is forced into the gasket to expand and stress the cylinder radially and circumferentially. The ends

of the specimen are free to allow for radial and longitudinal displacement. 
Methods have been employed to apply internal pressure at a very high loading rate to tubular specimens. Reed and Squires (1974) developed equipment for improving impact testing using shock pulses of gas to apply an "essentially infinite" loading rate of internal pressure to polymethylmethacrylate (PMMA). Bonfield and Datta (1974) used this shock tube technique to determine the impact fracture of cylindrical bovine bone specimens. The application of the internal pressure applied hoop stress in the cylinder. The pressure increased in successive pulses until the specimen fractured. This shock tube technique applies internal pressure at a very quick loading rate but does not allow a constant pressure to be maintained. A test method for slowly applying internal pressure was developed by Sakkers et al. (1995). They used a hydraulic system to determine the bursting pressure of cylindrical specimens machined from human femurs. Their device utilized a piston moving at a slow constant speed $(10 \mathrm{~mm} / \mathrm{min})$, gradually forcing water into a rubber hose to increase the pressure inside the bone cylinder until the specimen fractured. While the hydraulic system maintained constant pressure, the loading rate was slow.

The testing apparatus consisted of two aluminum end caps, two aluminum center bored hose plugs, a $62 \mathrm{~mm}$ length latex rubber hose (12.7 (inner diameter) x 3.2 (thickness) mm) (Kent Latex Products, Kent, OH, USA), four 9.5 x 1.58 (pitch) x 76.2 $\mathrm{mm}(3 / 8 \times 16 \times 3 \mathrm{inch})$ screws (tie rods) that were used to hold the device together, two plain carbon steel rectangular $66 \times 25.4 \times 6.4 \mathrm{~mm}$ end plate clamps to restrict the horizontal travel of the hose plugs, four $6.4 \times 0.907$ (pitch) $\times 76.2 \mathrm{~mm}(1 / 4 \times 28 \times 3 \mathrm{inch})$ threaded screws with matching nuts, a threaded stopper plug to prevent gas from leaking out of the hose, a pair of spacer washers measuring 19 (inner diameter) x 45 (outer diameter) $\mathrm{x}$ (thickness) $\mathrm{mm}$ and another pair measuring 25 (inner diameter) $\mathrm{x} 45$ (outer diameter) x 3 (thickness) $\mathrm{mm}$, and a hydraulic brake line for nitrogen supply with connections that measured approximately $0.92 \mathrm{~m}$. 


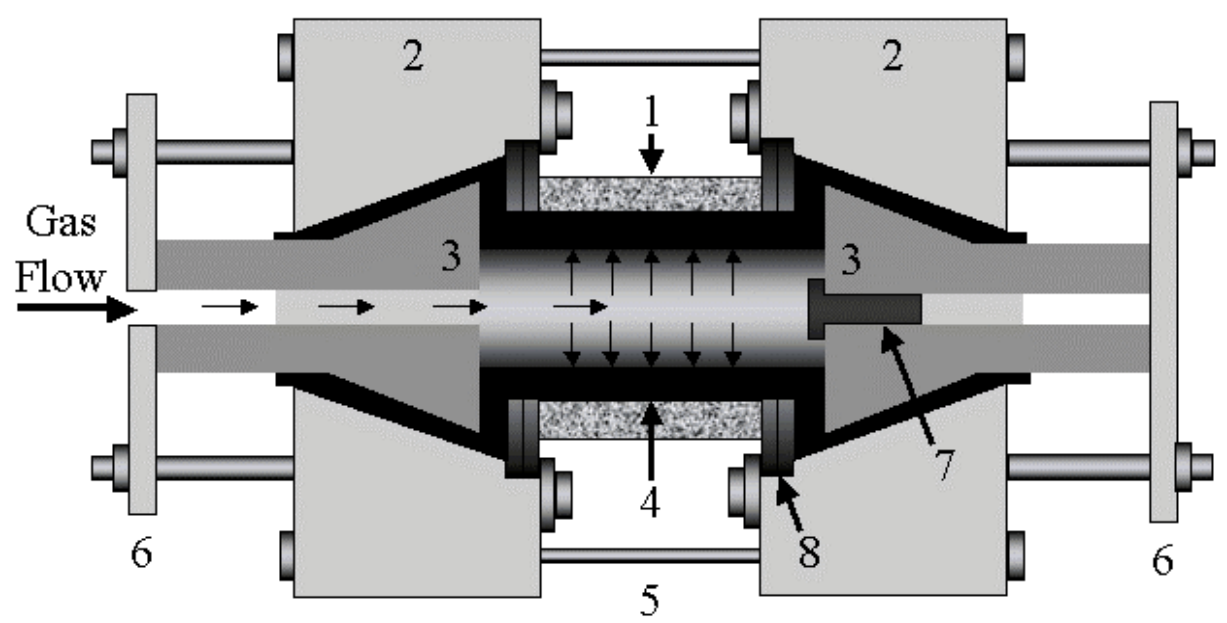

Figure 3.9 The test fixture with the cylindrical specimen (1), two aluminum end caps (2), two aluminum hose plugs (3), a $60 \mathrm{~mm}$ length latex rubber hose (4), four tie rods to hold the device together (5), two end plate clamps to restrict the horizontal travel of the hose plugs (6), a stopper plug to prevent gas from leaking out of the hose (7), and four spacer washers (8); cross-sectional view.

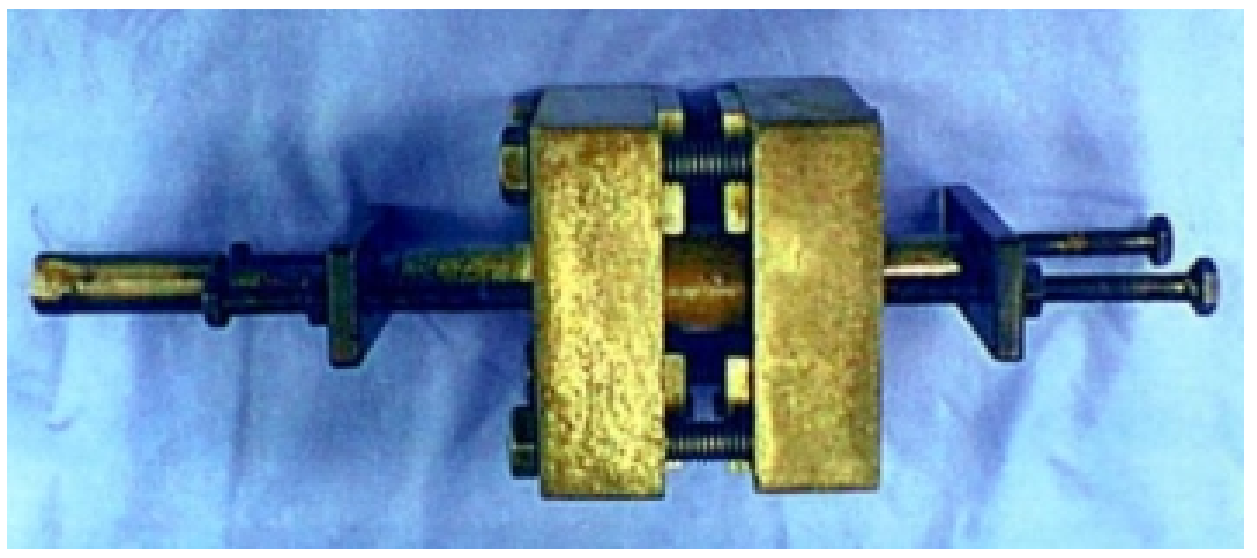

Figure 3.10 The test fixture with the bone specimen.

The specimen was placed within the test fixture and the lead wires were connected to the DAS. The test fixture included a latex hose that expands and conforms to the inner surface of the specimen during inflation. Lubricating, nongrease jelly was used at the interface between the latex and the specimen and between the specimen and the washers to minimize friction. After assembly, the tie rods were tightened until the washers rested against the specimen. The specimen was free of any axial load due to the end caps. 
Following the assembly of the testing apparatus, the nitrogen supply line was securely attached to the unconstrained hose plug. The other end of the steel supply line was connected to the pressure transducer. The entire test fixture including the bone specimen was then submerged in a saline bath that was heated and maintained at body temperature $37 \pm 0.56{ }^{\circ} \mathrm{C}$. The strain gages were monitored with an ohmmeter to be sure the specimen was the temperature of the saline before testing.

\subsection{Experiment equipment}

The test set-up consisted of a nitrogen cylinder, a dual stage high pressure regulator (Matheson Gas Products Inc., model 3030-580), an accumulator, a safety shutoff valve, a solenoid valve (Lexair, Inc., model 335804), a ventilation switch (Buyers Product Comp.), a small orifice (diameter $=0.015$ "), a pressure transducer (OMEGA Engineering Inc., model PX300, Stamford, CT) (excitation $=10 \mathrm{~V}$, gain $=166,30 \mathrm{mV}=$ 6.89 MPa (1000 psi)), a two way switch (Buyers Product Comp.), a self-regulating temperature hot water bath tank, automotive brake line, a computer data acquisition system, strain conditioning unit boards (Measurements Group, model 2311), a junction box, and the test fixture (Figure 3.11).

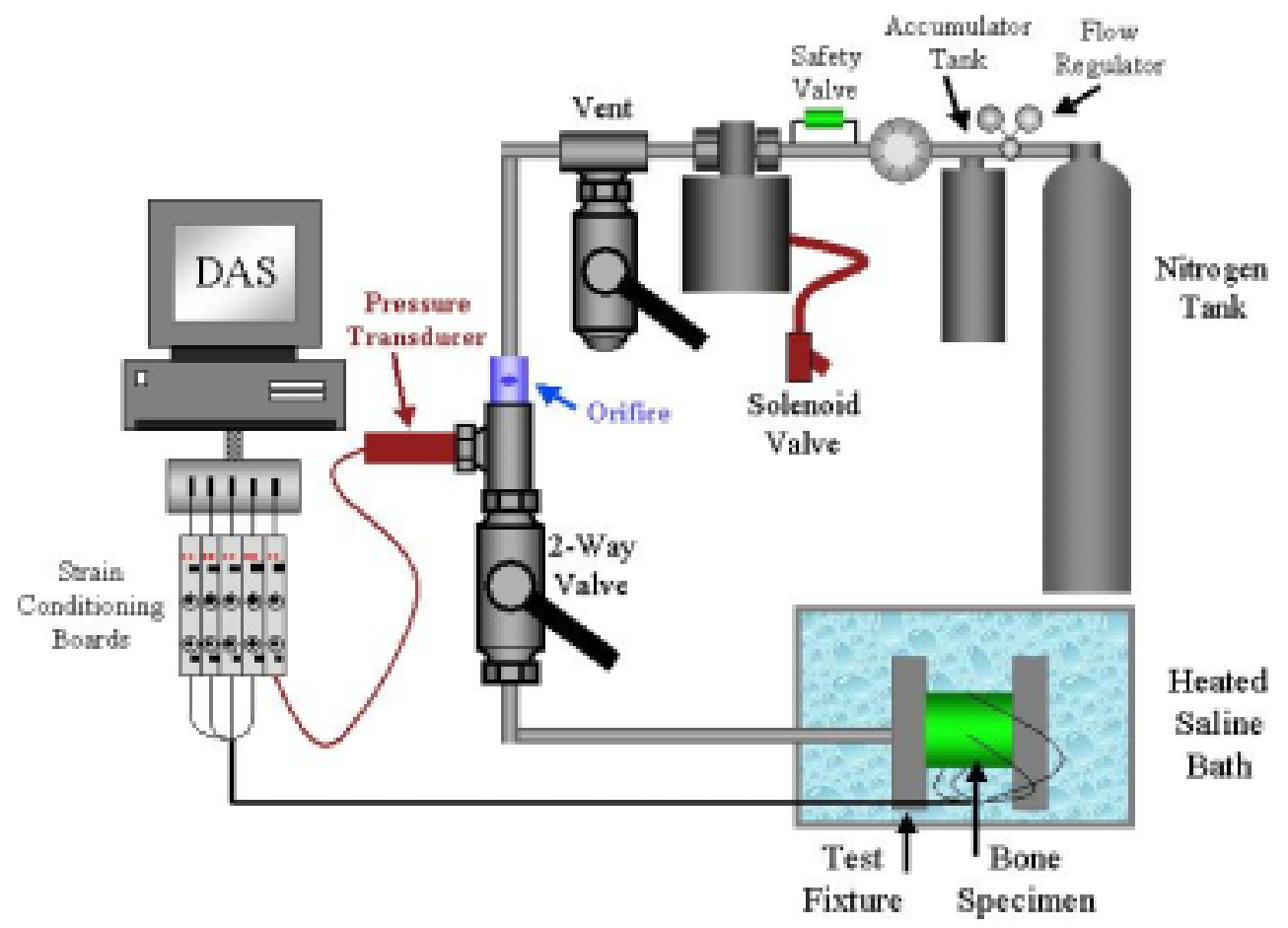

Figure 3.11 The experiment set-up. 
Nitrogen was fed through the regulator into the accumulator and then to the test fixture through the brake line. Nitrogen was used because it is inert and provides adequate pressure. To initiate the test, the valve was opened and the pressure from the accumulator was used to inflate the rubber hose within the specimen at a rate of approximately $1 \mathrm{MPa} /$ second. The pressure transducer with a working range between 0 and $6.89 \mathrm{MPa}(1000 \mathrm{psi})$, was calibrated comparing known pressures to the voltage output. Although the transducer was located upstream from the test apparatus, the rigid steel line had a negligible effect on pressure.

The temperature of the saline solution in the tank, $37 \pm 0.56{ }^{\circ} \mathrm{C}$, was maintained by a self-regulating temperature bath. This device contains an electrical circuit that switches the current to an electrical heater on and off to maintain the temperature of a system. The temperature sensor is a gas tube thermistor located in $0.9 \% \mathrm{NaCl}$. The thermistor was calibrated by comparing its resistance (observed on an ohmmeter) to a known temperature. The circuit was then adjusted to keep the preferred resistance (that corresponded to the desired temperature) a constant value.

The raw voltage for the pressure was converted using the following conversion factor: 1 volt $=1.37 \mathrm{MPa}(198.662 \mathrm{psi})$. A static zero was obtained with no internal pressure by zeroing the strain conditioning channels.

The latex hose did not cause nonlinear strains when the pressure was applied within an aluminum specimen (Figure 3.12). A sharp transition exists between the loading phase and the constant load phase. This behavior shows that the hose is not causing nonlinear strain behavior for this pressure range. 


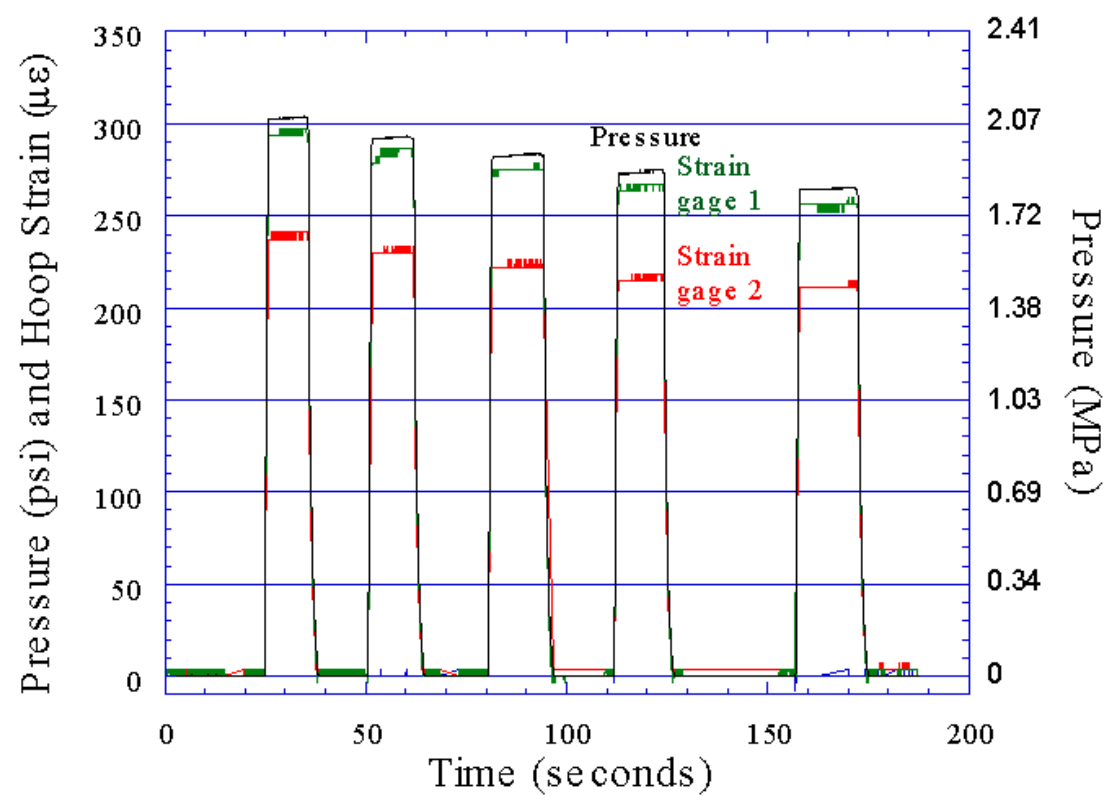

Figure 3.12 Pressure and microstrain versus time for an aluminum specimen (file=adjRate6b).

Various tests were conducted with the aluminum specimen to check for the effects of the latex hose and the repeatability of the test equipment using aluminum. The rate of loading was consistently repeatable using the equipment (test series "Rate" and "Hose").

\subsection{Strain Magnitudes on Whole Femurs}

The experimental model presented here was intended to simulate an idealized loading environment immediately after the insertion of a maximum fit and fill press-fit implant. The radial stress component from the implant to the bone is the only stress applied to the specimen. This radial load results in radial and hoop stress in the specimen. The radial stress also causes tensile hoop strain and radial compressive strain in the cylinder. To make a correlation between the two systems an understanding of the strain magnitudes in the intact femurs implanted with press-fit stems must be achieved.

There are many studies that measure strains on the outer surface of intact femurs with press-fit implants for the purpose of comparing the strain distribution before and after implant insertion and also to observe the effects of an in vivo loading environment. Zhou et al. (1990) measured a mean maximum strain of $1250 \mu \varepsilon$ (a range of $660-1834 \mu \varepsilon$ ) 
in the proximal-medial region due to a $2000 \mathrm{~N}$ load. An anatomically shaped stem design (Profile Hip, DePuy) provided a close fit to the distal and proximal canal and had rounded sections without corners. The strain measured was considered shear strain and the principal strain in the hoop direction was suggested to be higher. Jasty et al. (1994) loaded HGP collared components in single leg stance $(587 \mathrm{~N})$. Hoop strains at the proximal-medial cortex were approximately $459 \mu \varepsilon$. At the mid medial position, hoop strains were approximately $249 \mu \varepsilon$ and at the distal medial position, $330 \mu \varepsilon$. Murphy et al. (1984) measured principal tensile strain of $1189 \mu \varepsilon$ from a $225 \mathrm{~N}$ load of an Austin Moore stem. Otani et al. (1993) measured hoop strains of $499 \mu \varepsilon$ on the proximal medial cortex and $122 \mu \varepsilon$ on the distal medial cortex under a $2000 \mathrm{~N}$ axial load.

Oh and Harris (1978) measured outer strains from a $2012 \mathrm{~N}$ load due to cemented stems. Tensile strains along the mid to proximal femur measured approximately 500$1000 \mu \varepsilon$. Verdonschot and Huiskes (1998) measured approximately 50-500 $\mu \varepsilon$ for smooth and 50-700 $\mu \varepsilon$ for rough cemented stems at $7000 \mathrm{~N}$ load (during the first cycle of loading).

Jasty et al. (1993) measured the assembly strains produced during insertion of a straight stemmed, uncemented, collared HGP stem. Press-fitting the implant to a $0.5 \mathrm{~mm}$ interference fit produced hoop strains of $700-2400 \mu \varepsilon$ in the proximal femur and $100-$ $1100 \mu \varepsilon$ in the distal portion. Circumferential strains of $2400-6000 \mu \varepsilon$ were recorded prior to the development of fractures throughout the study.

Comparison between these studies is complicated due to different stems and different loading conditions. Based on the hoop strains from Jasty et al. (1993), a press-fit implant may induce strains of 100 to $2400 \mu \varepsilon$ along the cortex of the femur. Strains beyond $2400 \mu \varepsilon$ would be expected to fracture the bone. Therefore, applied pressure in this study should induce hoop strains in this same range to be considered a reasonable model for simulating press-fit insertion. 


\section{CHAPTER 4 \\ PRELIMINARY WORK}

The purpose of the preliminary work was to develop an experimental model and obtain preliminary data to study the behavior of bone in the transverse direction under conditions that simulate intramedullary loading. Using the experimental model, bone properties and failure were studied under controlled intramedullary conditions for the first time. The preliminary results support the hypothesis that bone exhibits creep behavior under intramedullary loading.

\subsection{Experimental Device for Applying Internal Pressure to Cylindrical Specimens (Brown et al., 2000a, Experimental Techniques)}

\subsubsection{Introduction}

Strains measured in aluminum and human femoral cortical bone specimens were compared to those calculated using thick-walled pressure vessel theory to validate the testing device. The goal was to develop a device to apply internal pressure with a controlled fast loading rate and maintain constant hoop stress in cylindrical specimens.

\subsubsection{The Cylindrical Specimens}

Two calibration specimens were used. The first, a hollow aluminum (6061-T6) thick walled cylinder was cut to a length of $19 \mathrm{~mm}$, with an inner radius, $\mathrm{r}_{\mathrm{i}}$, of $10 \mathrm{~mm}$ and an outer radius, $r_{0}$, of $11.6 \mathrm{~mm}$. The cylinder is considered "thick-walled" because the wall thickness to internal radius ratio $(0.17)$ is much greater than 0.05 , (Shigley and Mischke, 1989). Two strain gages (EA-06-125AD-120, Measurements Group, Inc., Raleigh, NC, USA) were applied to the outer surface of the cylinder along the middle between the cylinder ends. One gage was oriented to measure strain in the longitudinal direction, and the other was oriented to measure strain in the hoop direction (Figure 4.1). After bonding the strain gages to the aluminum cylinder, lead wires were soldered to the strain gage tabs and routed to strain conditioning units. 


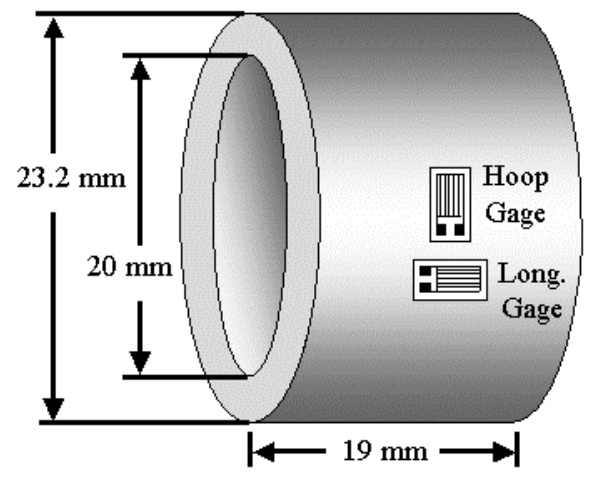

Figure 4.1 The aluminum cylinder with one longitudinal and one hoop strain gage.

Human femoral cortical bone was used for the second calibration specimen. A left and a right fresh femur were wet machined from an 86-year-old male cadaver. Two bulk sections from the mid-diaphysis of each femur were cut transversely to obtain specimens $19 \mathrm{~mm}$ long (Figure 4.2). Uniform right circular cylindrical specimens were machined to the following dimensions: $r_{i}=9.5 \mathrm{~mm}, r_{o}=11.9 \mathrm{~mm}$ and $19 \mathrm{~mm}$ long. The thickness of the bone specimens was $2.4 \mathrm{~mm}$ and considered thick-walled for the data analysis. Eight strain gages (EA-06-062DF-120) were applied at equal intervals around the circumference of the bone cylinder to measure hoop strain.

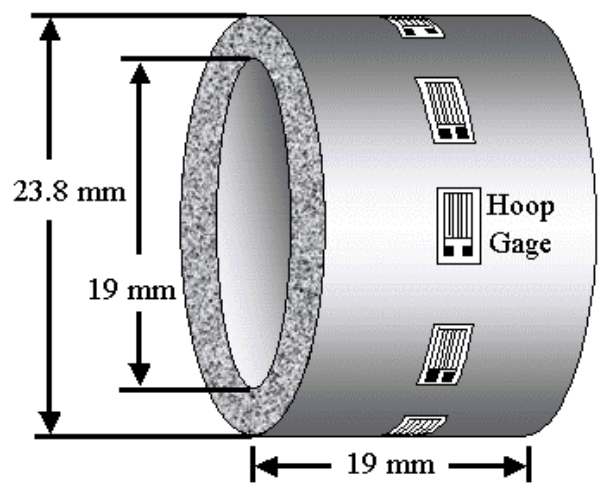

Figure 4.2. The bone cylinder with hoop strain gages along the mid-line of the specimen.

\subsubsection{Test Procedure}

For the aluminum specimen, a step increase in pressure allowed strains to be recorded at pressure settings through the measurable pressure range. This calibration curve also showed whether a linear relationship existed between the measured strain and the internal pressure. After the DAS was started, several seconds of baseline data were 
recorded. An internal pressure of $1.38 \mathrm{MPa}$ (200 psi) was then applied and the strain data was recorded for one minute to capture a strain measurement for the constant internal pressure of $1.38 \mathrm{MPa}$ (200 psi). The internal pressure was increased to $2.07 \mathrm{MPa}$ (300 psi) and strain data was collected at a constant pressure over a one-minute period. The pressure increased in increments between 0.55 and $0.83 \mathrm{MPa}(80-120 \mathrm{psi})$ for one-minute intervals until a pressure of $6.68 \mathrm{MPa}(970 \mathrm{psi})$ was reached. The internal pressure was not increased beyond $6.68 \mathrm{MPa}$ to prevent saturation of the amplifier. The DAS recorded hoop and longitudinal strain, the internal pressure and elapsed time (Figure 4.3). For each pressure increment, the mean strain was calculated from the strain recorded from the gages during the minute interval.

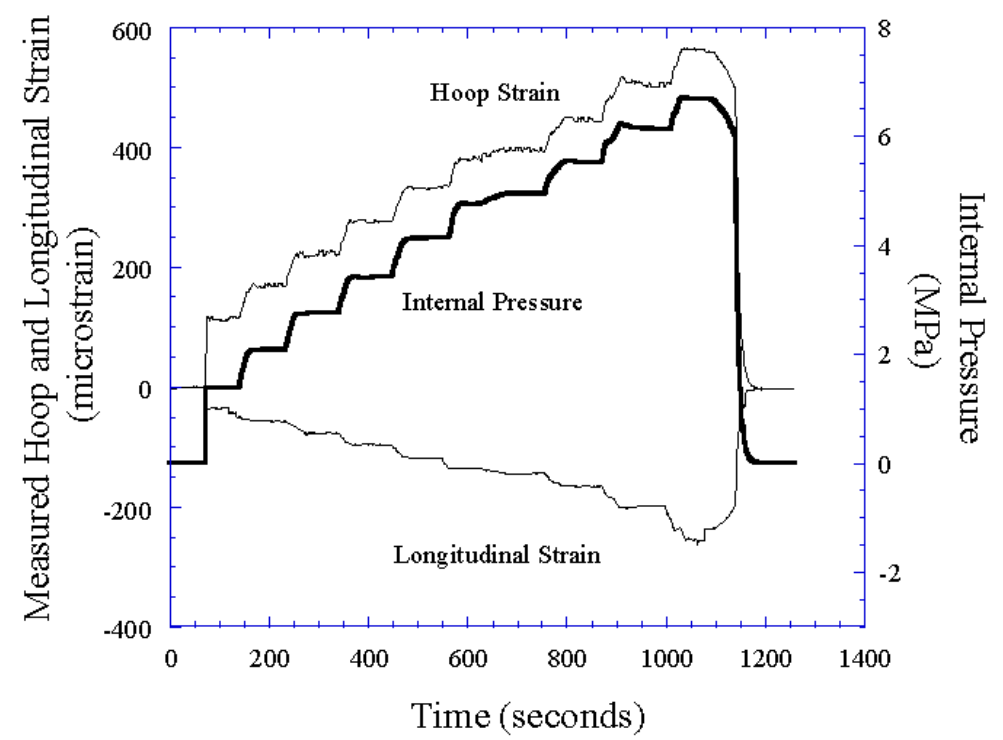

Figure 4.3 Measured hoop and longitudinal strain and internal pressure versus time for the aluminum specimen. A linear relationship between the measured strain and the internal pressure is evident.

A second test procedure was followed for the four bone specimens. Measured strains were recorded for a single pressure magnitude rather than over a range of pressures. Because multiple pressure magnitudes may fatigue or damage the bone specimen, only one low pressure setting was used. The specimens were loaded to 0.69 MPa (100 psi) in one second. The load was held constant for three hours. A span of 100 
seconds was chosen approximately 200 seconds after the internal pressure was applied when the strains had stabilized. The strains during this time were averaged for each gage.

\subsubsection{Data Analysis for the Aluminum Specimen}

To verify that the strains measured from the aluminum specimen during the experiment were accurate, measured values were compared to values calculated using thick-walled pressure vessel theory with the plane stress condition. The cylinder is assumed to be in a state of plane stress since no force is applied in the longitudinal direction yet axial deformation is allowed, therefore longitudinal stress, $\sigma_{z}$, is zero. The calculated stress value for hoop stress, $\sigma_{\theta}$, was determined for the outer surface of the specimen (Timoshenko and Goodier, 1970):

$$
\sigma_{\theta}=\frac{2 r_{i}^{2} P_{i}}{r_{o}^{2}-r_{i}^{2}}
$$

where $r_{i}$ and $r_{o}$ were the inner and outer radii of the cylinder and $P_{i}$ was the internal pressure. Radial stress, $\sigma_{\mathrm{r}}$, is zero on the surface of the cylinder. The constitutive equations for plane stress were used to calculate hoop strain, $\varepsilon_{\theta}$, radial strain, $\varepsilon_{\mathrm{r}}$, and longitudinal strain, $\varepsilon_{\mathrm{z}}$ :

$$
\begin{aligned}
& \varepsilon_{\theta}=\frac{1}{E} \sigma_{\theta} \\
& \varepsilon_{r}=\varepsilon_{z}=-\frac{1}{E} \nu \sigma_{\theta}
\end{aligned}
$$

where $\mathrm{E}=69 \mathrm{GPa}$ and $\mathrm{v}=0.33$ for aluminum (Byars et al., 1983).

\subsubsection{Data Analysis for the Femoral Bone Specimens}

Calculated values were determined for the bone specimens using thick-walled pressure vessel theory, the plane stress condition, and orthotropic material symmetry. Technical constants for human femoral bone as an orthotropic material were used (Ashman et al., 1984): $\mathrm{E}_{1}=12.0 \mathrm{GPa}, \mathrm{E}_{2}=13.4 \mathrm{GPa}, \mathrm{E}_{3}=20.0 \mathrm{GPa}, v_{12}=0.376, v_{13}=0.222$, $v_{23}=0.235, v_{21}=0.422, v_{31}=0.371, v_{32}=0.350$. The subscripts 1,2 and 3 represent the radial, hoop and longitudinal directions, respectively. 
The calculated stress value for $\sigma_{\theta}$ was determined using equation 4.1 where $\mathrm{P}_{\mathrm{i}}$ was $0.69 \mathrm{MPa}(100 \mathrm{psi})$. On the surface of the cylinder $\sigma_{\mathrm{r}}$ is zero and applying the plane stress assumption $\sigma_{z}$ is zero. The constitutive equation for human bone was used to determine the calculated strains:

$$
\varepsilon_{i}=S_{i 2} \sigma_{2}
$$

where $S_{\mathrm{i} 2}$ is the compliance matrix and $\sigma_{\mathrm{r}}=\sigma_{\theta}$.

\subsubsection{Results and Discussion}

Calculated strains on the outer surface of the aluminum specimen were compared to the measured strains to verify that the device applies hoop stress to the specimens. The calculated and measured hoop strains compare well (Table 4.1) (Figure 4.4). A line of fit between calculated and measured hoop strain $\left(\mathrm{R}^{2}=0.99\right)$ has a slope of 0.99 and a yintercept not significantly different than zero indicating no bias suggesting the strains are similar.

The calculated and measured longitudinal strains compare well for most of the pressure range (Table 4.1). The strains deviate during high-pressure settings, therefore, lower pressures from the first half of the pressure range will be used in the future. The reasons for the deviation are unknown, however, possibilities include the viscoelasticity of the rubber hose and friction at the rubber-aluminum interface. A line of fit between calculated and measured longitudinal strain $\left(\mathrm{R}^{2}=0.96\right)$ has a slope of 0.71 and a positive non-zero y-intercept.

The test fixture was designed to apply only internal pressure and no axial load to the specimen. Hoop and longitudinal strain measured by the strain gages for the aluminum specimen were used to determine Poisson's ratio by dividing longitudinal strain by hoop strain (Table 4.1). The mean ratio is $0.36 \pm 0.04$ for the entire pressure range and is greater than the material constant ratio for the aluminum material. However, for testing bone specimens the lower range of the internal pressures will be used where the measured ratio is similar to the material constant. This analysis shows that the test fixture has a usable pressure range up to $5.5 \mathrm{MPa}$. 
The measured hoop and longitudinal strains displayed a strong linear response with internal pressure (Figure 4.3). The observed linear behavior in both the hoop and longitudinal directions provides evidence for the accuracy of the test fixture.

The bone specimens were used to further assess the test fixture. The mean hoop strain for each specimen was determined from the 8 gages around each cortex (Table 4.2). The mean value of measured hoop strain for the four specimens was $173 \mu \varepsilon( \pm 77)$ and the calculated hoop strain was $179 \mu \varepsilon$, a 3\% difference. The small percent difference supports the use of the test fixture for human bone cylinders.

A text fixture was developed to apply internal pressure to cylindrical specimens. The agreement between the calculated and measured strain from the hoop and longitudinal gages from the aluminum specimen and the hoop gages from the human bone specimens suggest the test fixture does apply and maintain hoop stress to cylindrical specimens. This test fixture in the future may be used to model the transverse viscoelastic behavior of human bone.

Table 4.1 Internal pressure, measured and calculated hoop and longitudinal strain and the ratio of longitudinal to hoop strain for the aluminum specimen.

\begin{tabular}{cccccccc}
\hline $\begin{array}{c}\text { Int. } \\
\text { Pressure } \\
(\text { MPa })\end{array}$ & $\begin{array}{c}\text { Measured } \\
\text { Hoop } \\
\text { Strain } \\
(\boldsymbol{\mu \varepsilon})\end{array}$ & $\begin{array}{c}\text { Calculated } \\
\text { Hoop } \\
\text { Strain } \\
(\boldsymbol{\mu} \boldsymbol{\varepsilon})\end{array}$ & $\begin{array}{c}\% \\
\text { Diff }\end{array}$ & $\begin{array}{c}\text { Measured } \\
\text { Long. } \\
\text { Strain } \\
(\boldsymbol{\mu \varepsilon})\end{array}$ & $\begin{array}{c}\text { Calculated } \\
\text { Long. } \\
\text { Strain } \\
(\boldsymbol{\mu \varepsilon})\end{array}$ & $\begin{array}{c}\% \\
\text { Diff }\end{array}$ & $\begin{array}{c}\text { Poisson's } \\
\text { Ratio } \\
(\text { Long./ } \\
\text { Hoop } \\
\text { Strain) }\end{array}$ \\
\hline 1.39 & 112 & 116.58 & 4.03 & -35 & -38.47 & 9.75 & 0.31 \\
2.09 & 168 & 175.29 & 4.26 & -56 & -57.85 & 3.29 & 0.33 \\
2.76 & 222 & 231.48 & 4.17 & -77 & -76.39 & 1.01 & 0.35 \\
3.43 & 278 & 287.67 & 3.44 & -97 & -94.93 & 1.60 & 0.35 \\
4.14 & 334 & 347.22 & 4.89 & -118 & -114.58 & 2.69 & 0.35 \\
4.96 & 401 & 416.00 & 3.55 & -143 & -137.28 & 3.99 & 0.36 \\
5.54 & 446 & 464.64 & 4.10 & -164 & -153.33 & 6.94 & 0.37 \\
6.14 & 503 & 514.96 & 2.23 & -199 & -169.94 & 16.92 & 0.39 \\
6.70 & 567 & 561.93 & 0.93 & -253 & -185.44 & 36.27 & 0.45 \\
\hline
\end{tabular}




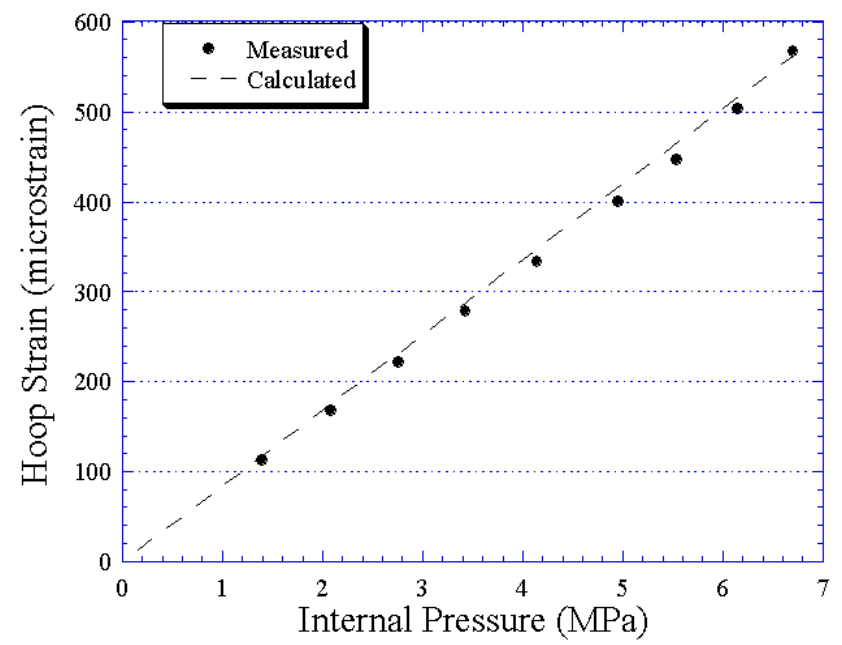

Figure 4.4 The relationships between measured $\left(\mathrm{R}^{2}=0.99\right)$ and calculated hoop strain versus internal pressure are similar for the aluminum specimen and suggests the testing device is accurate. Regression equation: Measured $\varepsilon_{\mathrm{H}}=83.76 \mathrm{P}_{\mathrm{i}}-8.98$

Table 4.2 Mean measured and calculated hoop strain for the femoral bone specimens at a pressure of $0.69 \mathrm{MPa}$.

\begin{tabular}{cccc}
\hline $\begin{array}{c}\text { Bone } \\
\text { Specimen }\end{array}$ & $\begin{array}{c}\text { Measured } \\
\text { Hoop Strain } \\
(\mu \varepsilon)\end{array}$ & $\begin{array}{c}\text { Calculated } \\
\text { Hoop } \\
\text { Strain } \\
(\mu \varepsilon)\end{array}$ & $\begin{array}{c}\% \\
\text { Difference }\end{array}$ \\
\hline 1 & 117 & & \\
2 & 283 & & \\
3 & 124 & & 3 \\
4 & 166 & 179 & \\
\hline Mean \pm St.D. & $173 \pm 77$ & & \\
\hline
\end{tabular}

Further validation of the test fixture was given by using another aluminum specimen. Tests were conducted that measured the strain change during the initial pressurization, thus allowing stress-strain curves to be analyzed. The internal pressure was converted into hoop stress and a plot of hoop stress versus hoop strain from the strain gage provided a slope of the initial linear curve, hoop stiffness. The stiffness for this type of aluminum specimen is $69 \mathrm{GPa}$ (10E6 psi). During one test (Hose8), the first strain gage measured an average hoop stiffness of $70 \pm 3.9 \mathrm{GPa}(10.15 \mathrm{E} 6 \pm 0.56 \mathrm{psi})$ and the second gage measured $91 \pm 6.2 \mathrm{GPa}(13.22 \mathrm{E} 6 \pm 0.90 \mathrm{psi})$ for a pressure range from 0.31 
to $1.58 \mathrm{MPa}$ (45 to $229 \mathrm{psi}$ ) (Figure 4.5). During the second test (Hose9), the first gage measured a stiffness of $58 \pm 5.6 \mathrm{GPa}(8.48 \mathrm{E} 6 \pm 0.81 \mathrm{psi})$ and the second gage measured $76.5 \pm 7.6 \mathrm{GPa}(11.11 \mathrm{E} 6 \pm 1.10 \mathrm{psi})$ for a pressure range from 0.28 to $2.58 \mathrm{MPa}$ (40 to 375 psi). The difference in the stiffness values between gages is most likely due to the variation in the thickness of the specimen, a uniform thickness was assumed.

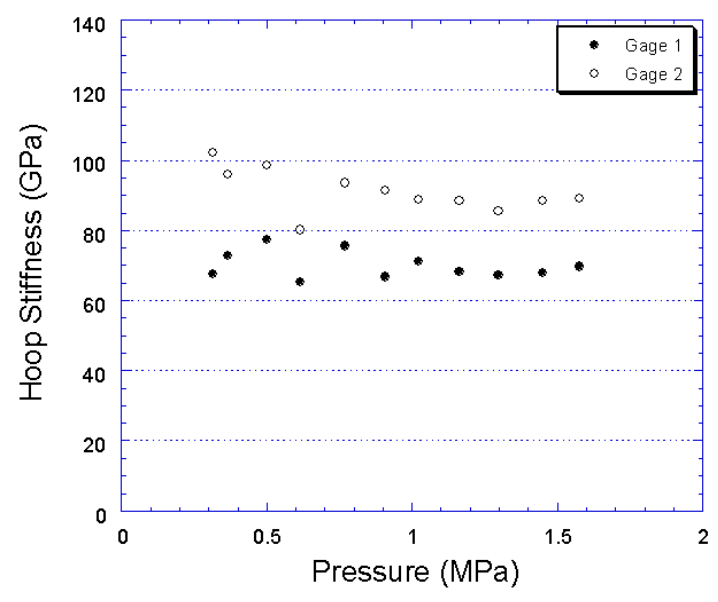

(A)

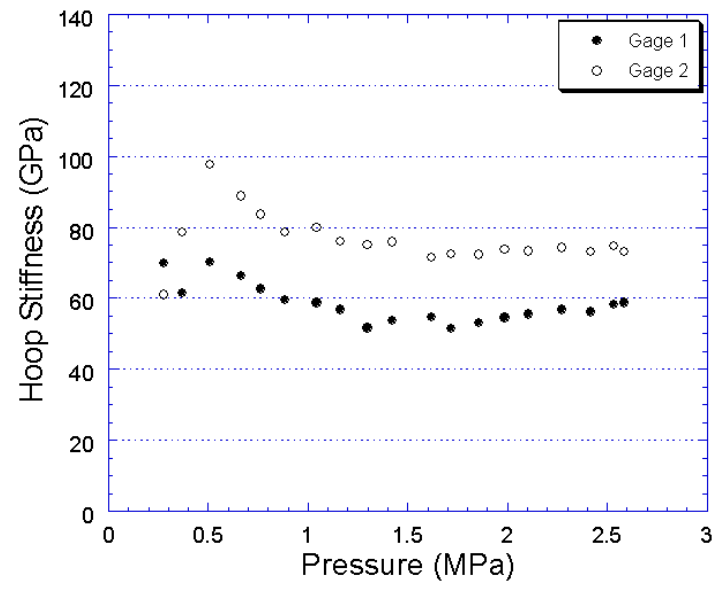

(B)

Figure 4.5 Hoop stiffness over the pressure range for the first (A) and second (B) aluminum test using a thinner aluminum specimen. The variation between the two gages is most likely due to the uneven specimen thickness.

\subsection{Circumferential Strain Around Femoral Cylinders Due to Simulated Press-Fit} Loading (Brown et al., 1998)

\subsubsection{Introduction}

A key to preventing failure of a total hip replacement system is to better understand the reaction of cortical bone to the forces applied by the implant. Press-fit arthroplasty relies on the implant applying a radial force to the proximal femur resulting in hoop strain in the bone. Many studies have examined the structural reaction of bone by collecting strain from the cortical bone surface of the femur with a cementless press-fit implant. Little is known, however, about the reaction of bone as a material to radial forces. Preliminary work was performed to study the strain distribution around the 
circumference of the bone cylinders subjected to internal pressure. We hypothesized that the elastic modulus in the hoop direction should vary between cortical regions.

\subsubsection{Materials and Methods}

To reduce variability, four bone specimens were wet machined from an 86 year old male cadaver. Eight strain gages were applied at equal intervals around the circumference of each cylinder whose thickness was $2.4 \mathrm{~mm}$ (Figure 4.6). The specimens were loaded to $0.69 \mathrm{MPa}$ (100 psi) in one second and held at constant pressure for three hours. A span of 100 seconds was chosen approximately 200 seconds after the internal pressure was applied (Figure 4.7). The strains during this time were stable and the mean strain determined for each gage.

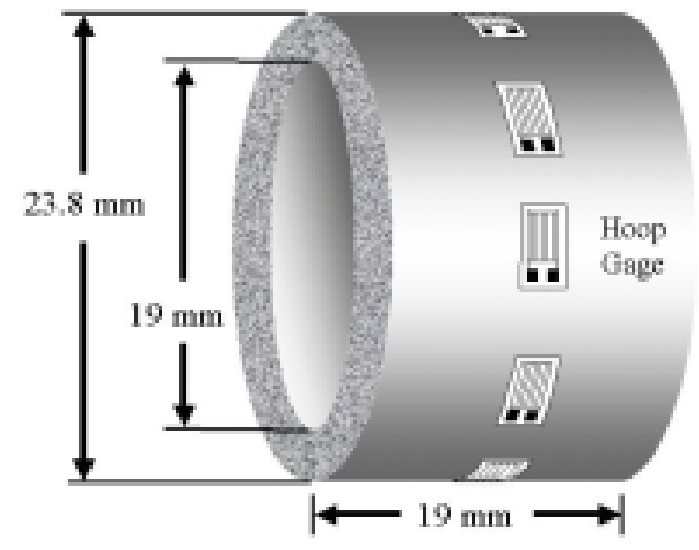

Figure 4.6 The bone cylinder, with a thickness of $2.4 \mathrm{~mm}$ and gages to measure hoop strain.

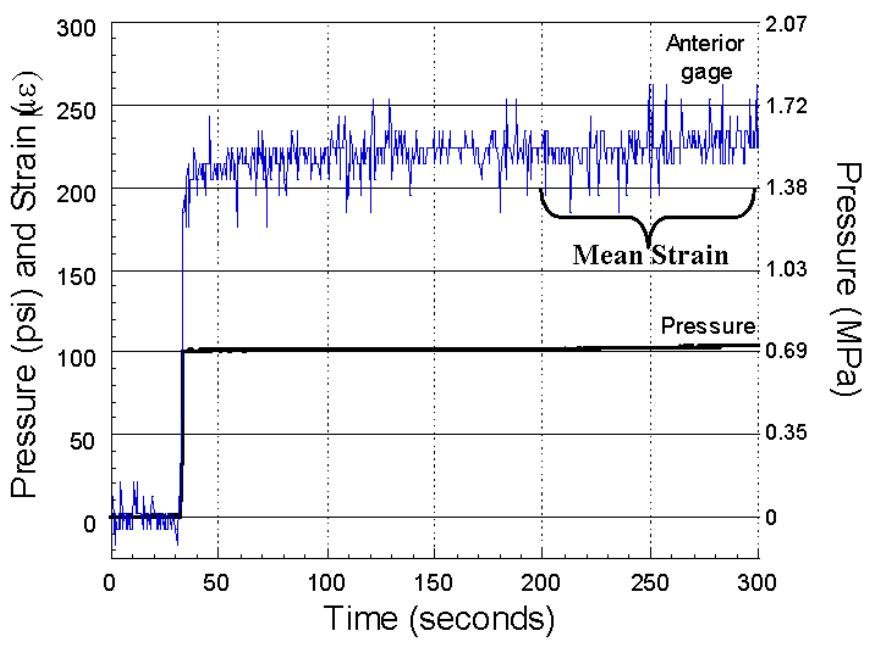

Figure 4.7 The mean strain was determined from the 100 second span. 


\subsubsection{Results}

The mean strain value and standard error for each of the eight cortical regions was determined using the four specimens (Table 4.3). In the case of the posterior and posterior-lateral regions, only three specimens could be used due to gage-failure of one specimen. No statistically significant differences were found among the hoop strain magnitudes of each cortical region, likely due to the low number of specimens tested. When the mean strains are plotted in their respective locations on a polar plot, there appears to be symmetry in the strain distribution, where the anterior and the posterior cortical regions have higher strains than the medial and lateral regions (Figure 4.8).

Table 4.3 Mean hoop strain and standard error of each cortical region.

\begin{tabular}{|l|c|c|}
\hline \multicolumn{1}{|c|}{ Cortical Region } & Mean strain $(\boldsymbol{\mu \varepsilon})$ & Standard Error \\
\hline Posterior & 321.05 & 80.24 \\
\hline Posterior-Lateral & 171.38 & 94.56 \\
\hline Lateral & 143.41 & 94.56 \\
\hline Anterior-Lateral & 117.19 & 80.24 \\
\hline Anterior & 180.86 & 80.24 \\
\hline Anterior-Medial & 123.76 & 80.24 \\
\hline Medial & 126.63 & 80.24 \\
\hline Posterior-Medial & 186.29 & 80.24 \\
\hline
\end{tabular}

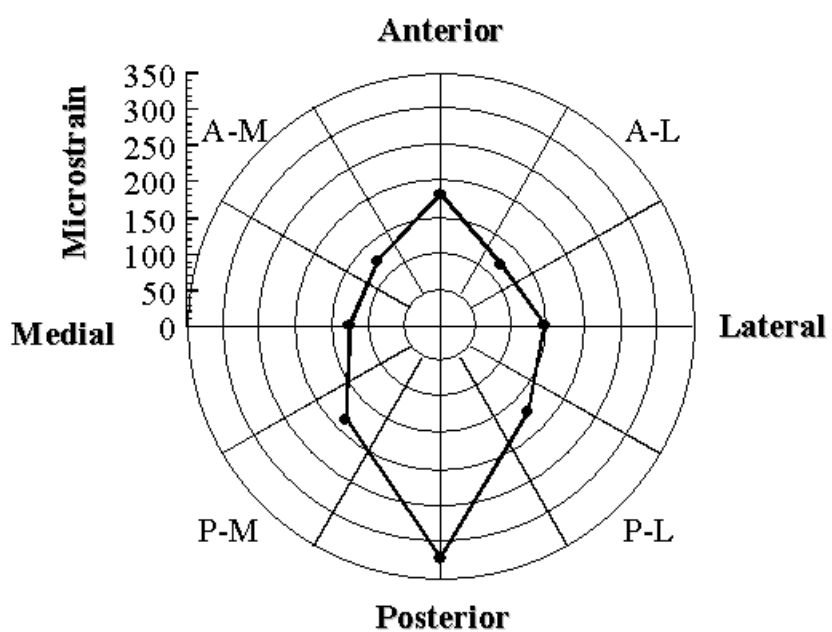

Figure 4.8 Mean hoop strain from each cortical region around the circumference of the four femoral cylinders. 


\subsubsection{Discussion}

This study is unique in determining the strain pattern around a uniform cylinder of femoral bone rather than around the irregular femoral shaft or using flat-testing specimens. Mechanical properties such as the elastic modulus in the longitudinal direction have been shown to vary in different cortical regions around the circumference of the femoral shaft (Ashman et al., 1984; Rho et al., 1995). Our data suggest that based on our limited number of specimens, there is a difference in the hoop elastic modulus as indicated by strain variations where anterior and posterior cortices may undergo more strain than the medial and lateral cortices. The variation in strain may be a result of morphologic differences such as porosity within the bone cortex. It has been reported that the medial and lateral femoral cortices have a higher longitudinal stiffness than the anterior and posterior cortices (Ashman et al., 1984) and the highest bending stress for the femur occurs along the medial and lateral cortices (Van Buskirk, 1989). Lower strains in the medial and lateral cortices would, therefore, be expected due to a higher stiffness of the bone at these locations.

\subsection{Transverse Creep Response in Human Femoral Bone (Brown et al., 1999)}

\subsubsection{Introduction}

Creep behavior due to transverse loads such as those applied by press-fit stems has not been studied. The elastic properties and hoop stresses in cortical bone hold the press-fit implant in place until osseointegration (Blaha, 1998). If cortical bone exhibits creep (time-dependent) behavior in the transverse direction the host bone may deform sufficiently that initial fixation is lost before bone apposition occurs. Depending on the stress level, deformation may progress until fracture. The objective of this research was to study the short-term transverse creep behavior of human cortical bone. An understanding of this time-dependent behavior may be useful to improve the clinical success of total hip replacements. 


\subsubsection{Materials and Methods}

Four right circular cylindrical specimens with a wall thickness of $2.4 \mathrm{~mm}$ were wet machined from an 86 year old male cadaver. A test fixture applied constant internal pressure to the inner surface of the specimen in a saline solution bath at $37^{\circ} \mathrm{C}$. Specimen \#1, 3 and 4 were loaded at a constant internal pressure of 2.07 (300 psi), 2.01 (292 psi) and 1.68 MPa (243 psi), respectively, until the specimen failed. Specimen \#2 fractured during the application of the internal pressure. Eight strain gages (grid dimension $1.57 \mathrm{x}$ $1.57 \mathrm{~mm}$ ) were oriented to measure hoop strain at equal intervals around the circumference of the cylinder. The test fixture, including the bone specimen, was submerged in a saline solution bath at body temperature for the duration of the test.

\subsubsection{Results}

The load level was not sufficient to cause bone fracture upon loading in three of four specimens. Despite the different amounts of internal pressure used for each specimen, the specimens failed at different lengths of time where the specimen loaded with the least amount of pressure took the shortest length of time to fail (Table 4.4). Failure of some gages due to electrical shorts to the saline prevented strain measurements from being made at all locations. Based on the remaining gages, the anterior cortex appears to be strain the most in these three specimens (Figures 4.9, 4.10 and 4.11). The noise evident in the raw data was a result of electrical interference and not due to strain variations. A fourth order polynomial was used to fit the data of a single strain gage (specimen $\# 3$, anterior) $\left(r^{2}=0.843\right)$ that includes only the creep response of the specimen, not the initial elastic deformation. The equation that defines hoop strain as a function of time is:

$$
\varepsilon_{\mathrm{H}}=723.3+0.98 \mathrm{t}+0.10 \mathrm{t}^{2}-5.47 \mathrm{E}-3 \mathrm{t}^{3}+6.85 \mathrm{E}-5 \mathrm{t}^{4}
$$

where $t$ is the elapsed time in minutes. 
Table 4.4 Constant internal pressure magnitudes and time-to-failure for the bone specimens.

\begin{tabular}{|c|c|c|}
\hline $\begin{array}{c}\text { Specimen } \\
\#\end{array}$ & $\begin{array}{c}\text { Constant Internal } \\
\text { Pressure } \\
\text { MPa (psi) }\end{array}$ & $\begin{array}{c}\text { Time to Failure } \\
\text { (seconds) }\end{array}$ \\
\hline 1 & $2.07(300)$ & 31800 \\
\hline 2 & - & - \\
\hline 3 & $2.01(292)$ & 3700 \\
\hline 4 & $1.68(243)$ & 64 \\
\hline
\end{tabular}

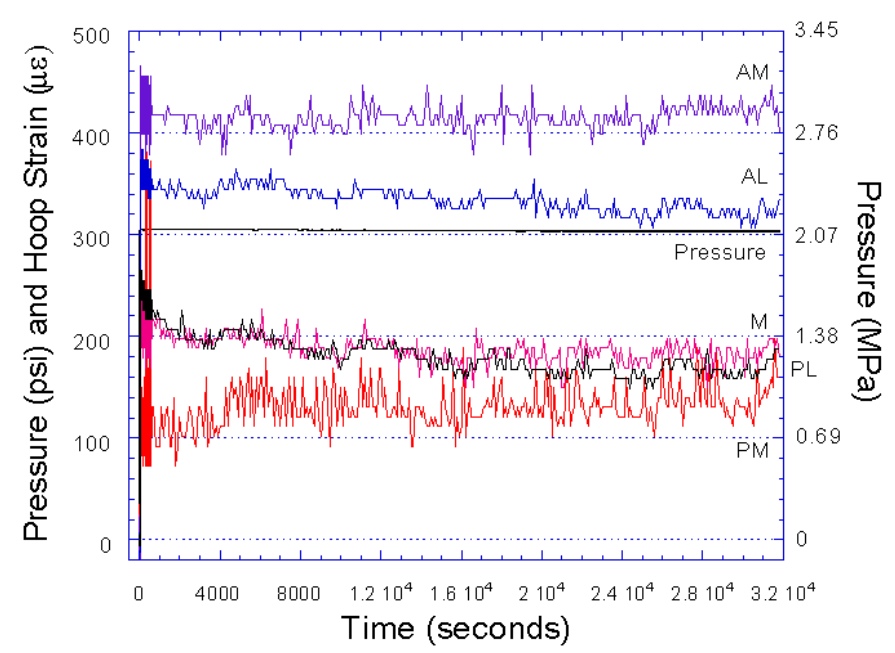

Figure 4.9 Specimen \#1, failure occurred between 31800 and 31900 seconds at a pressure of 2.07 MPa (300 psi).

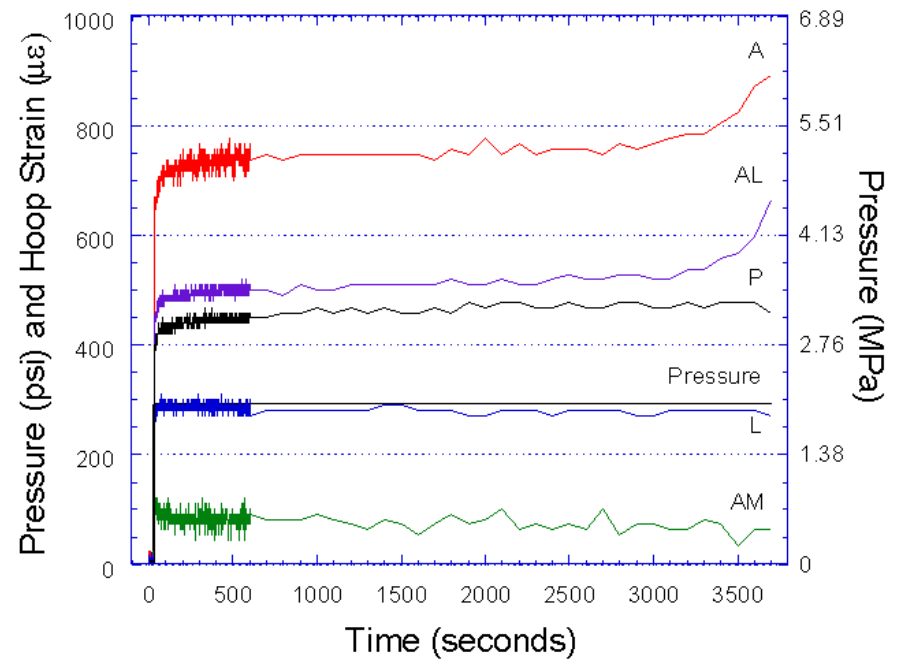

Figure 4.10 Specimen \#3, failure occurred between 3700 and 3800 seconds at a pressure of 2.01 MPa (292 psi). 


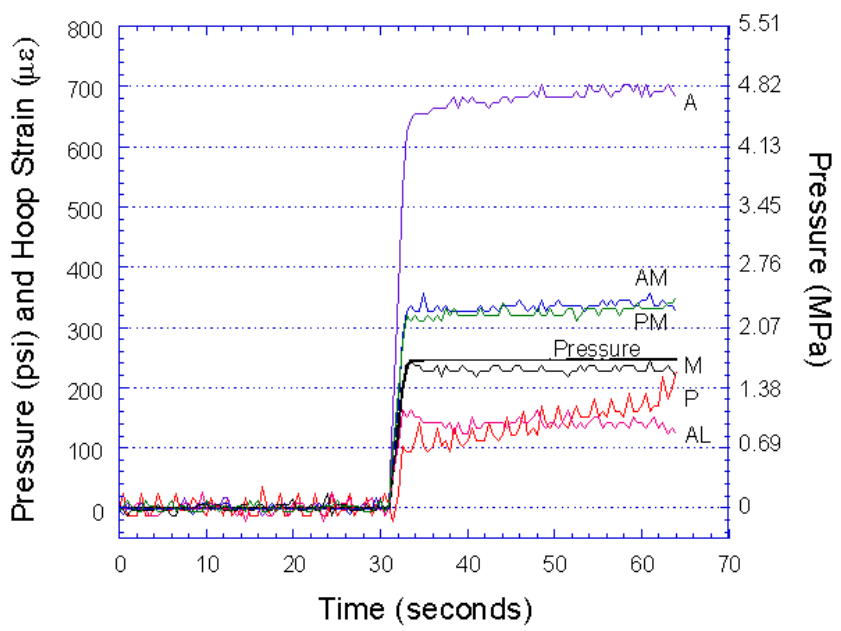

Figure 4.11 Specimen \#4, failure occurred between 64 and 64.5 seconds at a pressure of $1.68 \mathrm{MPa}(243 \mathrm{psi})$.

\subsubsection{Discussion}

The results of this study indicate that cortical bone experiences creep behavior under radial loading and exhibits the three basic characteristic stages prior to fracture. Stage 1 is the initial response to the internal load and the creep rate decreases. During stage 2 the creep rate remains constant as the specimen continues to deform. The creep rate increases as the specimen deforms until failure during stage 3 (Figure 4.12). This creep behavior occurs at the time of load application and may affect the initial fixation and stability of a femoral implant. The importance of a reliable waterproofing material for the gages for long-term exposure to saline is also obvious.

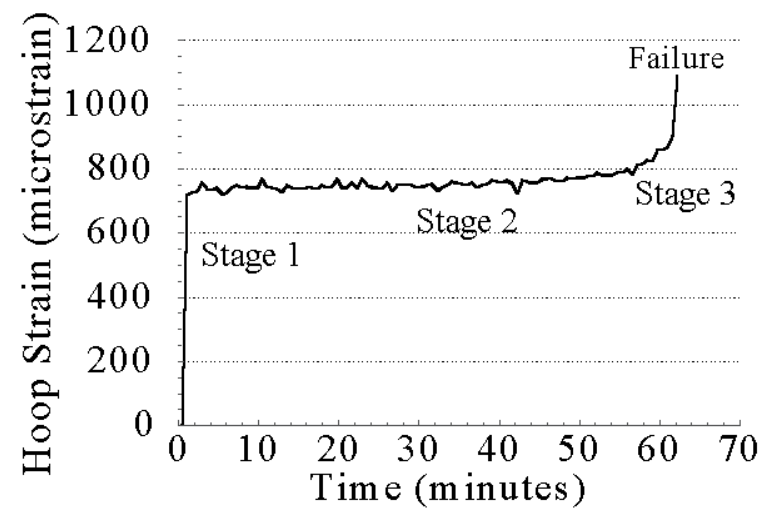

Figure 4.12 Transverse elastic and creep strain of cortical bone, specimen \#3 anterior gage, subjected to an internal pressure of $2 \mathrm{MPa}$ (292 psi). A cubic spline was used to fit the data. 
Not every gage should be expected to display all three stages of creep. In some cases the third stage happens quickly enough that it occurs in between the data sampling frequency. In other cases, a hinge mechanism appears to be occurring where a specific quadrant has a quicker rate of tensile hoop strain and as a result causes compressive strain in the quadrants opposite or adjacent. A good example of this phenomenon occurred for specimen \#3 (Figure 4.10). The anterior strain gage displayed the third stage of creep. During this phase, the posterior gage recorded compressive strain.

Press-fitted implants generate high hoop stresses in the bone that may result in a time-dependent response. The deformation of the host bone may result in a loss of initial fixation of the stem or may cause creep-fracture of the bone. The creep response of bone to the implantation of a prosthesis is a possible contributing factor to the loss of implant stability before osseointegration.

Postoperative fractures are occasionally unrecognized intraoperative fractures (Schwartz, et al., 1989; Penenberg, 1998; Kelley, 1994). The tensile hoop and compressive radial stresses due to the internal pressure are greater along the inner surface of the cylinder then the outer surface. These high stresses may induce creep fracture along the inner surface before the outer surface. The results suggest that the creep fracture observed in this study may account for the appearance of fractures postoperatively that have previously been unrecognized intraoperative fractures.

Stable intraoperative fractures (Schwartz et al., 1989) recognized on the periosteal surface of the femur may disguise the potentially unstable creep fracture at the endosteal surface. Thus stable intraoperative fractures may become unstable due to the creep behavior of cortical bone. 


\section{CHAPTER 5 \\ SHORT-TERM CREEP TESTS}

\subsection{Introduction}

The degree of press-fit between a press-fit hip implant and the endosteal surface of the femoral canal is important for achieving initial fixation which leads to improved load transfer and less micromotion (Ring, 1978; Engh, 1983; Morscher, 1983; Robertson et al., 1988; Poss et al., 1988). After the implant is inserted into the femur, a sufficient amount of force is needed between the implant and bone to maintain initial implant stability. The elastic response in bone holds the implant until new bone growth occurs and supports the implant (Blaha, 1998). Excessive force at the time of insertion may result in high assembly strains and cause fractures (Jasty et al., 1993; Kyle, 1999). The radial load may cause the bone to experience creep behavior, time-dependent deformation under constant load. Creep deformation may threaten initial implant stability by reducing the press-fit before new bone growth can occur.

The objective of this study was to determine hoop creep thresholds of femoral bone subjected to an intramedullary radial load to understand how bone deforms due to stresses that may occur as a result of a press-fit hip prosthesis. The relationships between hoop stress and hoop creep strain, creep strain rate and permanent strain were examined to understand how femoral cortical bone responds to an intramedullary radial load.

\subsection{Materials and Methods}

Ten fresh human femora were obtained from 8 male and 2 female cadavers (mean age $39 \pm 13$ years) (Brown et al., 2001). From these femora a total of 15 right circular cylinders $19 \mathrm{~mm}$ in length, with a $19 \mathrm{~mm}$ inner wall diameter and a mean wall thickness of $2.01 \pm 0.05 \mathrm{~mm}$ were wet machined. Four unidirectional strain gages were mounted on the outer surface of the specimen on the anterior, posterior, lateral and medial aspects; all gages were oriented to measure hoop strain. 
Table 5.1 Distribution of specimens with respect to gender and age. Note that specimen tests 1, 2 and 3 were practice specimens.

\begin{tabular}{|c|c|c|c|c|}
\hline Number & Specimen \# & Test \# & Gender & $\begin{array}{c}\text { Age } \\
\text { (years) }\end{array}$ \\
\hline 1 & $6184 \# 3$ & 4 & Male & 45 \\
\hline 2 & $6372 \# 4$ & 5 & Male & 22 \\
\hline 3 & $96174 \# 3$ & 6 & Male & 66 \\
\hline 4 & $96174 \# 5$ & 7 & Male & 66 \\
\hline 5 & $96174 \# 1$ & 8 & Male & 66 \\
\hline 6 & $96174 \# 2$ & 9 & Male & 66 \\
\hline 7 & $76181 \# 2$ & 10 & Male & 33 \\
\hline 8 & $6182 \# 2$ & 11 & Male & 40 \\
\hline 9 & $976089 \# 2$ & 12 & Male & 48 \\
\hline 10 & $976090 \# 1$ & 13 & Male & 33 \\
\hline 11 & $6184 \# 1$ & 14 & Male & 45 \\
\hline 12 & $976136 \# 3$ & 15 & Female & 24 \\
\hline 13 & $76070 \# 1$ & 16 & Male & 45 \\
\hline 14 & $6372 \# 2$ & 17 & Male & 22 \\
\hline 15 & $6369 \# 3$ & 18 & Female & 38 \\
\hline
\end{tabular}

\subsubsection{Setting the Pressure}

There were main components involved in correctly and safely applying the pressure for these tests, they involved the solenoid valve ("solenoid") which opened and closed with an electrical switch, a vent valve ("vent") which allowed the pressure to be released from the line, and the 2-way switch valve ("valve") which opened and closed allowing the pressure to load the specimen. 


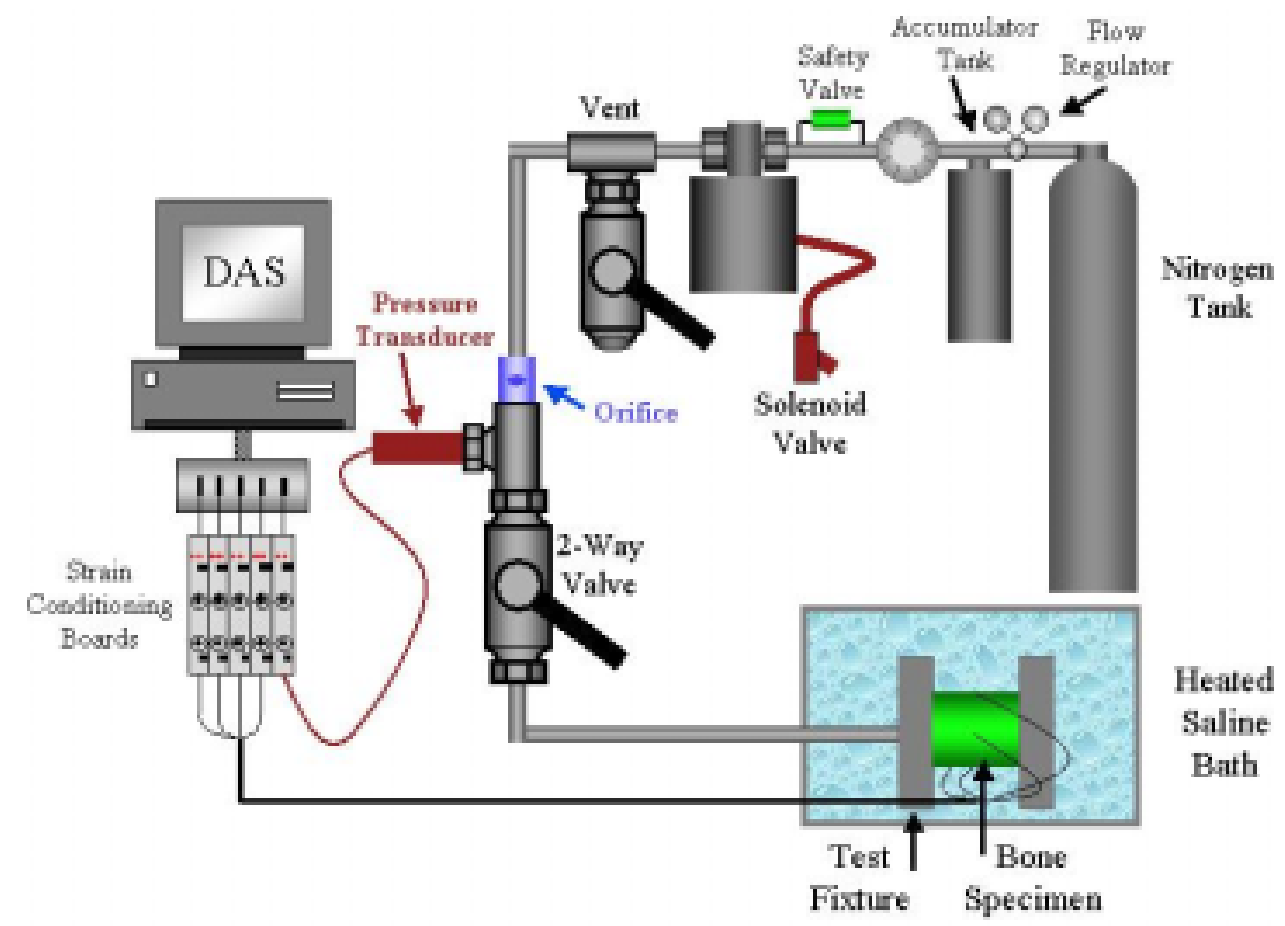

Figure 5.1 The testing equipment for the short-term creep tests.

The initial desired pressure of $0.345 \mathrm{MPa}$ (50 psi) was set. This minimum pressure was needed for stable strain measurements and proper sealing of the pressurized hose within the test fixture. The DAS was started on the computer; the valve, vent and solenoid were closed. The constant flow regulator was set to the desired working pressure and the accumulator was charged via the regulator to the same pressure. The solenoid was opened and the pressure entered the brake line while the pressure transducer measured the pressure monitored on the computer. If the pressure was too low, the solenoid was closed, the vent was opened and closed releasing the pressure only within the brake line and the accumulator was charged again. If the pressure was too high, the vent was opened and the solenoid valve was opened and closed to allow the pressure to bleed down to the desired amount.

Once the pressure was set to the pre-determined amount, the test was ready to begin and the solenoid and vent closed and the valve was opened. At the correct time, the solenoid was opened allowing the pressure to load the specimen. When 60 seconds has passed, the solenoid was turned off and the vent was quickly opened to de-pressurize the specimen. The valve was closed during the two minutes that the specimen was at zero- 
pressure, preventing any internal load to specimen while the pressure was increased in $0.103 \mathrm{MPa}$ (15 psi) increments (Figure 5.2). The vent was closed and the solenoid valve was opened to set the pressure as described above. The maximum pressure allowed was $3.06 \mathrm{MPa}$ (444 psi) before the solenoid valve would not operate.

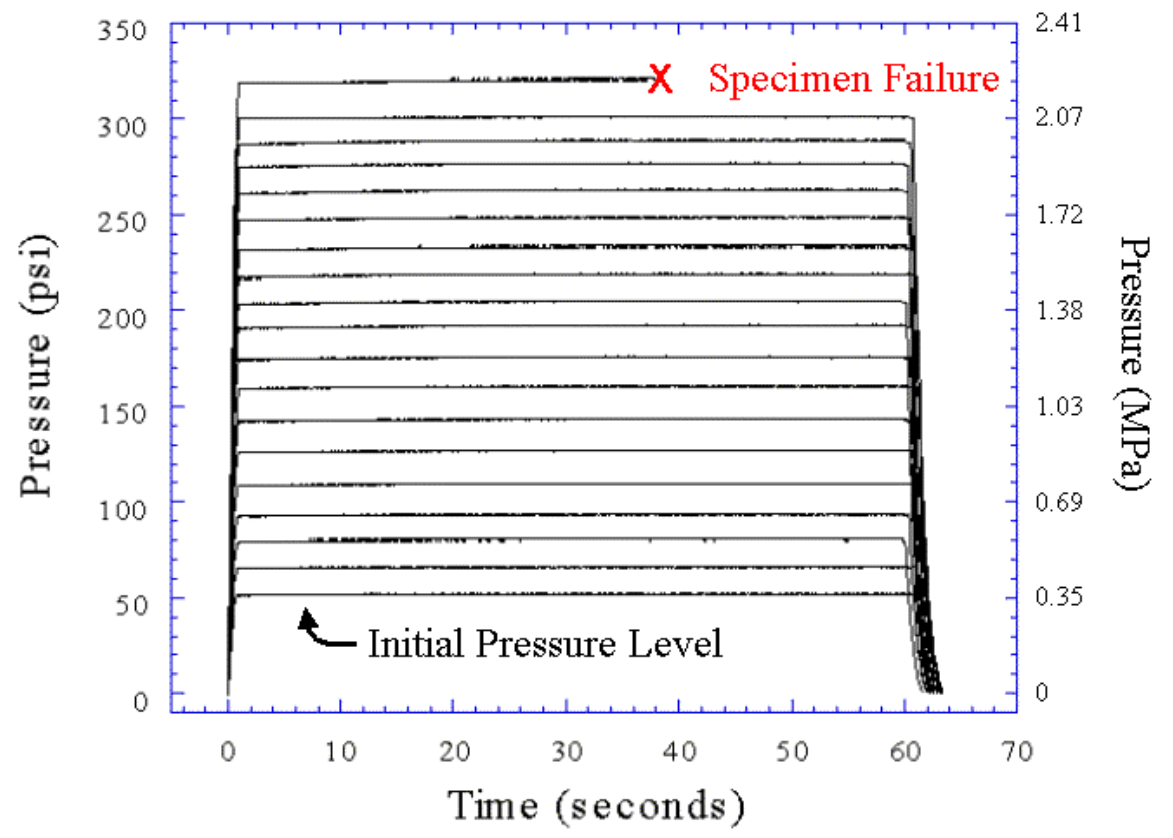

Figure 5.2 A pressure of $0.345 \mathrm{MPa}$ (50 psi) was applied initially for the first phase of one minute of pressure and increased during each cycle. These are the pressure levels for one specimen where the final pressure level resulted in failure (Test 8).

\subsubsection{Multi-Phase Testing Protocol}

The loading procedure followed a cycle of four phases for each specimen (Utenkin and Sveschnikova, 1973; Melnis et al., 1979; Fondrk et al., 1988; Nicolella et al., 1998). Fondrk et al. (1988) used a testing method that combined the standard constant stress creep test (Christensen, 1982) and a multiple loading sequence (Cristescu and Suliciu, 1982). In their study each cycle consisted of the following: a one second ramp-up in tensile load, a 1 minute hold at constant load, a 1 second ramp-down, and a 1 minute hold at zero-load. This cycle was repeated increasing the tensile load by $25 \mathrm{MPa}$ for the first few cycles and by $6 \mathrm{MPa}$ for the specimens close to the yield stress until failure. An additional minute was used between cycles to download the data to a computer disk, therefore the total time the specimen was at zero-load was 2 minutes. Utenkin and 
Sveschnikova (1973) used a similar loading cycle for compressive tests. Specimens were compressed for 90 minutes at two stress levels, $50 \%$ and $90 \%$ of the elasticity limits, then left unloaded for 60 minutes. Melnis et al. (1979) conducted tensile creep tests at 6 load levels ranging from $0.2-0.7 \sigma_{\mathrm{u}}$ (or higher until failure) in increments of $0.1 \sigma_{\mathrm{u}}$. A 200 minute hold was maintained during the constant stress phase, then the load was released and the specimen relaxed for 200 minutes. The specimen was held at zero-load for 1000 minutes as the residual creep deformation was measured in between loading phases. The phases were continued with increasing hold stress until failure. Nicolella et al. (1998) used a similar multiple loading cycle test regime to conduct a constant stress creep test. The load cycle involved a ramp-up in load until a specified global strain level. The stress level was maintained for 2 minutes, released, and then zero-load was maintained for 2 minutes. This cycle was maintained at incrementally higher strain levels until failure.

For this study a multi-phase loading protocol was followed to observe the creep behavior. During phase 1, pressure was applied in less than 2 seconds. The pressure was held constant for one minute during phase 2, released during phase 3 , and the specimen remained at zero pressure for 2 minutes during phase 4 while strain was monitored (Figure 5.3).

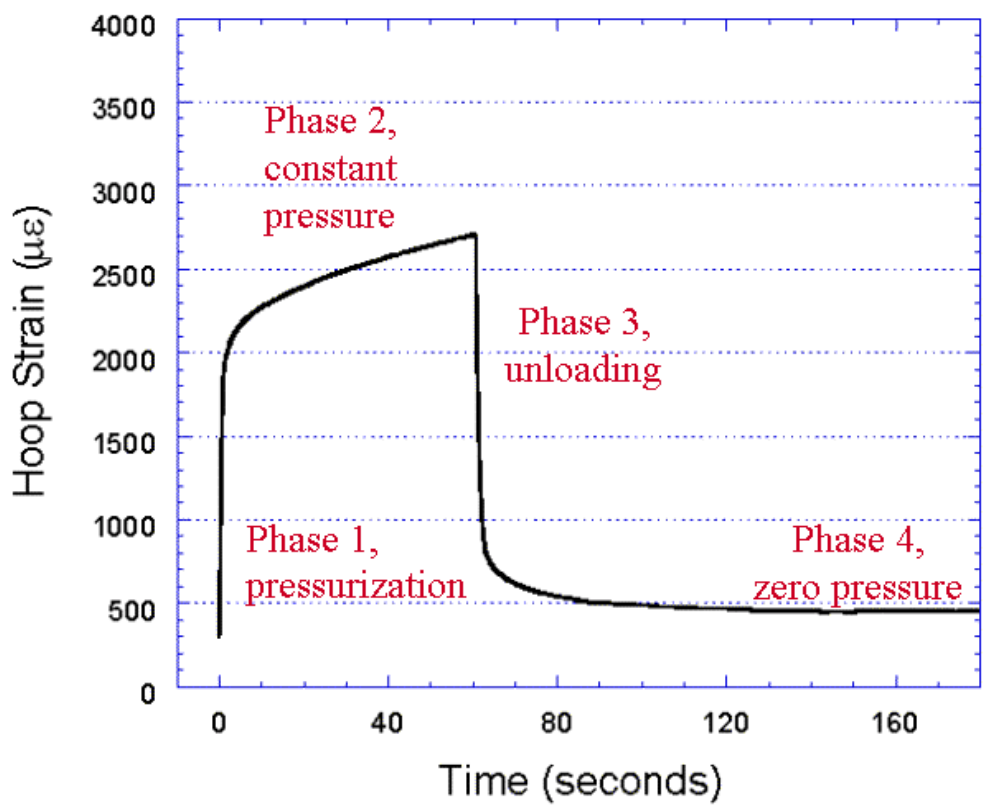

Figure 5.3 The four phases of the loading cycle: phase 1, pressurization; phase 2, constant pressure; phase 3 , unloading; phase 4 , zero pressure (Test 8 , final cycle). 
For each successive cycle, the pressure increased $0.103 \mathrm{MPa}(15 \mathrm{psi})$ until the specimen failed or a constant pressure of $3.06 \mathrm{MPa}$ (444 psi) was used (Figure 5.4). Several parameters were obtained from each test including hoop stiffness, creep strain, creep rate and permanent strain.

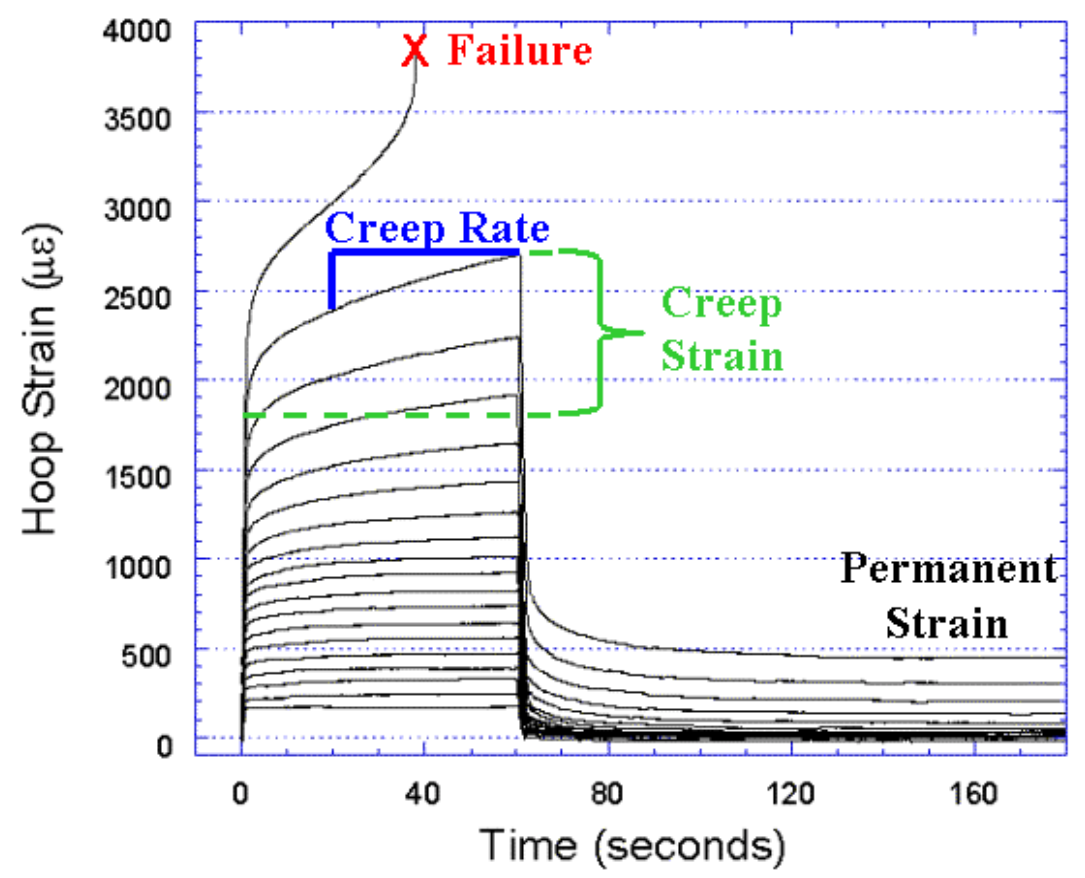

Figure 5.4 The measured parameters from the short-term tests: creep strain, creep rate and permanent strain.

\subsubsection{Morphological and Statistical Analysis}

After the specimens were tested, a section $200 \mu \mathrm{m}$ thick cross section was cut directly under the strain gage location at each quadrant using an Isomet low-speed saw (Buehler Ltd). Each section was polished and stained with hematoxylin-eosin for morphological evaluation. Morphology measurements were conducted with an image analysis system (Optimus, Edmonds, WA) and a transmitted light microscope (Olympus, Edmonds, WA) under 40x magnification. Average measurements were taken over three different fields that spanned the length of the strain gage to reduce the measurement errors (Martin et al., 1980). The abbreviations for morphological parameters followed the standard nomenclature for bone histomorphometry (Parfitt et al., 1987). In a selected 
field, the total area of the selected field (Tt.Ar), the total area of haversian and Volkmann's canals and other pores (Tt.Po.Ar), the total area of osteons including haversian canals (Tt.On.Ar), and the total number of osteons (Tt.N.On) were measured using Optimus and recorded in a spreadsheet file. Osteonal measurements were made following the criteria of Wang (1997), Martin et al. (1980) and Currey (1988). Percent porosity, Po (\%), was calculated as:

$$
P o(\%)=\frac{T t \cdot P o \cdot A r}{T t . A r}
$$

Percent osteonal area, On.Ar (\%), was calculated as:

$$
\operatorname{On} \cdot \operatorname{Ar}(\%)=\frac{T t \cdot O n \cdot A r}{T t \cdot A r}
$$

Osteon density, On.Dn $\left(\mathrm{mm}^{-2}\right)$, was calculated as:

$$
\operatorname{On} \cdot \operatorname{Dn}\left(\mathrm{mm}^{-2}\right)=\frac{T t . N . O n}{T \cdot . A r}
$$

Average area of a single osteon, s. $\operatorname{On} . \operatorname{Ar}\left(\mathrm{mm}^{2}\right)$, was calculated as:

$$
\text { s.On.Ar }\left(\mathrm{mm}^{2}\right)=\frac{T_{t} \cdot . \mathrm{On} \cdot \mathrm{Ar}}{T_{t . N .} \cdot O n}
$$

Statistical analysis was performed using JMP (SAS Institute, Cary, NC). Statistical significance was set as $p<0.05$ or less. The following statistical procedures were used such as analysis of variance (ANOVA) for examining differences in bone quadrants and repeatability, linear fitting including the $95 \%$ confidence interval in the procedure to determine the thresholds, standard least squares for general linear models (including both quantitative and character variables) to examine differences in creep strain, creep rate, permanent strain and normalized hoop stress at different bone quadrants, stepwise regression analysis for screening variables for inclusion in least squares procedures, and curve fitting using the nonlinear platform for obtaining the coefficient and exponent of a power law function.

Using ANOVA, the repeatability of the morphological parameters was determined. The porosity and osteon density measurements were very repeatable with an interclass correlation coefficient (ICC) of 0.90 and 0.96 , respectively. The percent of osteon area and the average area of a single osteon were less repeatable with ICC equal to 0.52 and 0.76 , respectively. 


\subsection{Hoop Stiffness (Instantaneous Modulus)}

During the loading phase, the pressure transducer recorded the pressure increase. Internal pressure was converted to hoop stress using thick-walled pressure vessel theory (equation 2.5), and the strain gages measured hoop strain. A linear regression of the hoop stress against hoop strain was used to model the initial behavior and determine the hoop stiffness or instantaneous modulus (Figure 5.5). This value of hoop stiffness, $\mathrm{E}_{\mathrm{H}}$, was then used to normalize the stress level in an attempt to account for differences in the bone from the different donors.

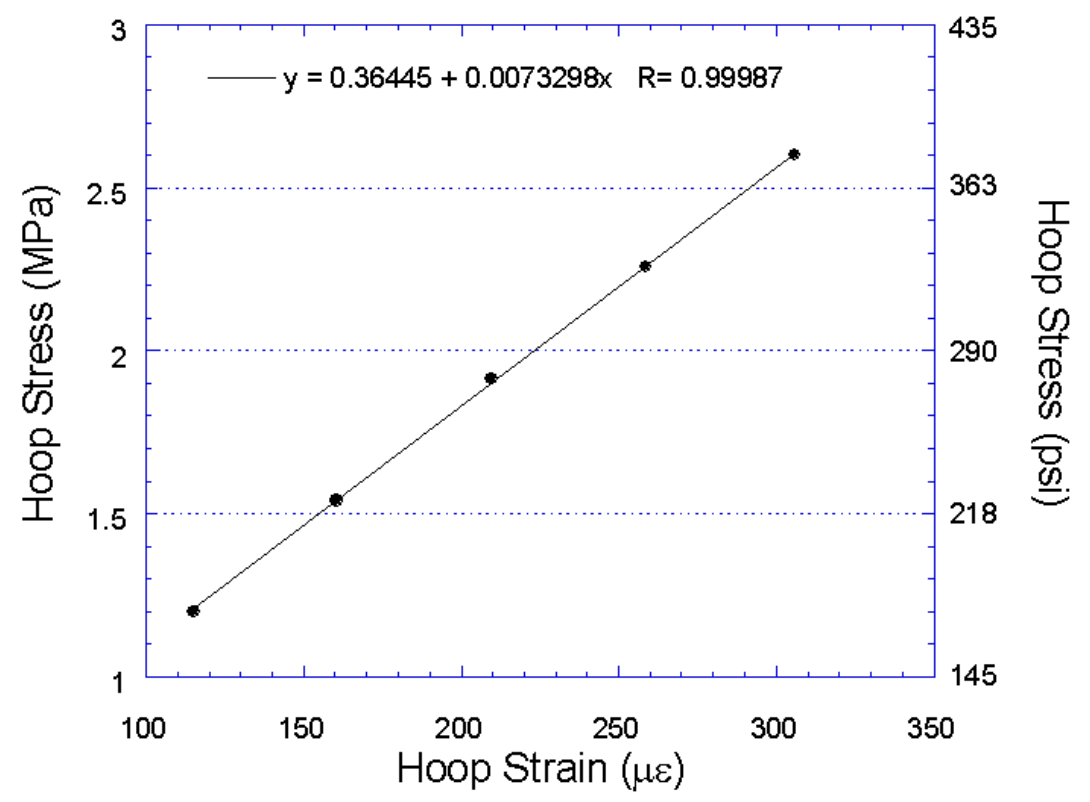

Figure 5.5 A typical hoop stress-strain curve for the loading portion of a sample stress level (Test 8d). The hoop stiffness is $7.33 \mathrm{GPa}(1.06 \mathrm{E} 6 \mathrm{psi})$.

Normalizing stress with stiffness is commonly used to account for differences in bone quality. Caler and Carter (1989) determined the longitudinal elastic modulus to normalize the stress levels used for each tensile and compressive creep specimen in their study. Bowman et al., (1994) followed a similar procedure to normalize trabecular bone specimens to study compressive creep behavior. Mauch et al. (1992) determined a value of stiffness of each bovine and deer antler creep specimen before testing. Longitudinal stiffness was used to normalize the stress levels for each test. Bowman et al. (1999) determined the elastic modulus by loading each specimen in tension under displacement 
control at $0.025 \mathrm{~mm} / \mathrm{s}$, with nine preconditioning cycles between approximately $0.0-5.0$ percent strain before a final ramp to obtain the initial portion of the linear region of the stress-strain curve. The elastic modulus was used to normalize the stress levels for each specimen. Normalizing stresses with the elastic modulus was also done by Bowman et al. (1998) reducing scatter by accounting for inter-specimen variations in density and microstructure.

The hoop stiffness values at each quadrant from hoop stress-strain curves during the initial pressurization $(0.345 \mathrm{MPa}(50 \mathrm{psi})$ ) were determined (Table 5.2) for comparison to other studies that report hoop stiffness. Stiffness values were significantly different between the medial and posterior quadrants $(\mathrm{p}=0.04)$ and between the medial and lateral quadrants $(\mathrm{p}=0.038)$ (Figure 5.6). All values were within the range (8.51-13.4 $\mathrm{GPa}$ ) of previously conducted studies that are typically used as references in the literature for stiffness of cortical bone (Cowin et al., 1987) (Table 5.3). A mean initial hoop stiffness of $10.52 \mathrm{GPa}$ was determined from all the stiffness values.

Table 5.2 The mean initial hoop stiffness values from each quadrant determined from the initial pressurization of $0.345 \mathrm{MPa}(50 \mathrm{psi})$ for each specimen ( $\mathrm{n}=15$ for each quadrant).

\begin{tabular}{|c|c|c|}
\hline Quadrant & $\begin{array}{c}\text { Initial Hoop Stiffness } \\
\text { Mean } \pm \text { St.D. }(\text { GPa })\end{array}$ & $\begin{array}{c}\text { Max, Min } \\
\text { (GPa) }\end{array}$ \\
\hline Posterior & $9.77 \pm 1.86$ & $13.34,7.04$ \\
\hline Medial & $11.71 \pm 2.97$ & $20.16,8.64$ \\
\hline Anterior & $10.82 \pm 1.48$ & $13.41,8.65$ \\
\hline Lateral & $9.80 \pm 1.63$ & $12.48,6.46$ \\
\hline Average & $10.52 \pm 2.17$ & $20.16,6.46$ \\
\hline
\end{tabular}




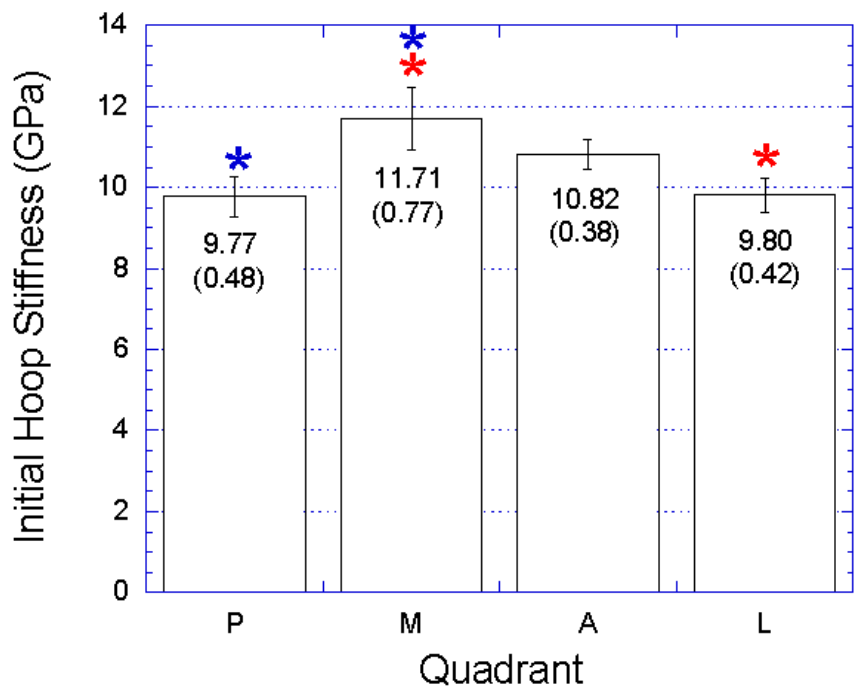

Figure 5.6 The initial hoop stiffness for each quadrant (pressure $=0.345 \mathrm{MPa}(50 \mathrm{psi})$ ) for all specimens $(\mathrm{n}=15)$. Significant differences were found between the medial and posterior quadrants $(\mathrm{p}=0.04)$ and between the medial and lateral quadrants $(\mathrm{p}=0.038)$. The mean initial hoop stiffness is included with the standard error in parenthesis.

Table 5.3 Strain rates and corresponding stiffness values measured in different studies while loading specimens in tension.

\begin{tabular}{|c|c|c|c|c|c|c|}
\hline Study & Bone & Symmetry & Method & $\begin{array}{c}\text { Strain } \\
\text { Rate } \\
(\varepsilon / \mathbf{s})\end{array}$ & $\begin{array}{c}\text { Hoop } \\
\text { Stiffness } \\
\text { (GPa) }\end{array}$ & $\begin{array}{c}\text { Long./ } \\
\text { Radial } \\
\text { Stiffness } \\
\text { (GPa) }\end{array}$ \\
\hline $\begin{array}{l}\text { Crowninshield } \\
\text { and Pope } \\
(1974)\end{array}$ & $\begin{array}{c}\text { Bovine } \\
\text { tibia }\end{array}$ & - & $\begin{array}{l}\text { Mechanical } \\
\text { Testing }\end{array}$ & $\begin{array}{c}0.00167- \\
1.67\end{array}$ & $\approx 2$ & $\approx 12 /-$ \\
\hline $\begin{array}{l}\text { Reilly and } \\
\text { Burstein } \\
(1975)\end{array}$ & $\begin{array}{l}\text { Human } \\
\text { femur }\end{array}$ & $\begin{array}{l}\text { Transversely } \\
\text { Isotropic }\end{array}$ & $\begin{array}{l}\text { Mechanical } \\
\text { Testing }\end{array}$ & $\begin{array}{l}0.02- \\
0.05\end{array}$ & 11.5 & $17.0 / 11.5$ \\
\hline $\begin{array}{c}\text { Yoon and } \\
\text { Katz (1976) }\end{array}$ & $\begin{array}{l}\text { Human } \\
\text { femur }\end{array}$ & $\begin{array}{l}\text { Transversely } \\
\text { Isotropic }\end{array}$ & Ultrasound & - & 18.8 & $27.4 / 18.9$ \\
\hline $\begin{array}{l}\text { Wright and } \\
\text { Hayes (1976) }\end{array}$ & $\begin{array}{l}\text { Bovine } \\
\text { femur }\end{array}$ & 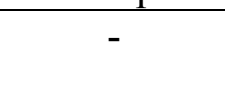 & $\begin{array}{l}\text { Mechanical } \\
\text { Testing }\end{array}$ & $\begin{array}{l}0.0053- \\
0.053\end{array}$ & - & $19.9-23 /-$ \\
\hline $\begin{array}{c}\text { Knets et al. } \\
\text { (1977) }\end{array}$ & $\begin{array}{c}\text { Human } \\
\text { tibia }\end{array}$ & Orthotropic & $\begin{array}{l}\text { Mechanical } \\
\text { Testing }\end{array}$ & - & 8.51 & $18.4 / 6.91$ \\
\hline $\begin{array}{l}\text { Ashman et al. } \\
\text { (1984) }\end{array}$ & $\begin{array}{l}\text { Human } \\
\text { femur }\end{array}$ & Orthotropic & Ultrasound & - & 13.4 & $20.0 / 12.0$ \\
\hline $\begin{array}{l}\text { Current } \\
\text { Study }\end{array}$ & $\begin{array}{l}\text { Human } \\
\text { femur }\end{array}$ & - & $\begin{array}{l}\text { Mechanical } \\
\text { Testing }\end{array}$ & $\begin{array}{r}0.00017 \\
-0.0023\end{array}$ & 10.5 & $-/-$ \\
\hline
\end{tabular}


For a single specimen, a hoop stress-strain curve shows a decrease in stiffness due to damage during repeated load cycles (Figure 5.7).

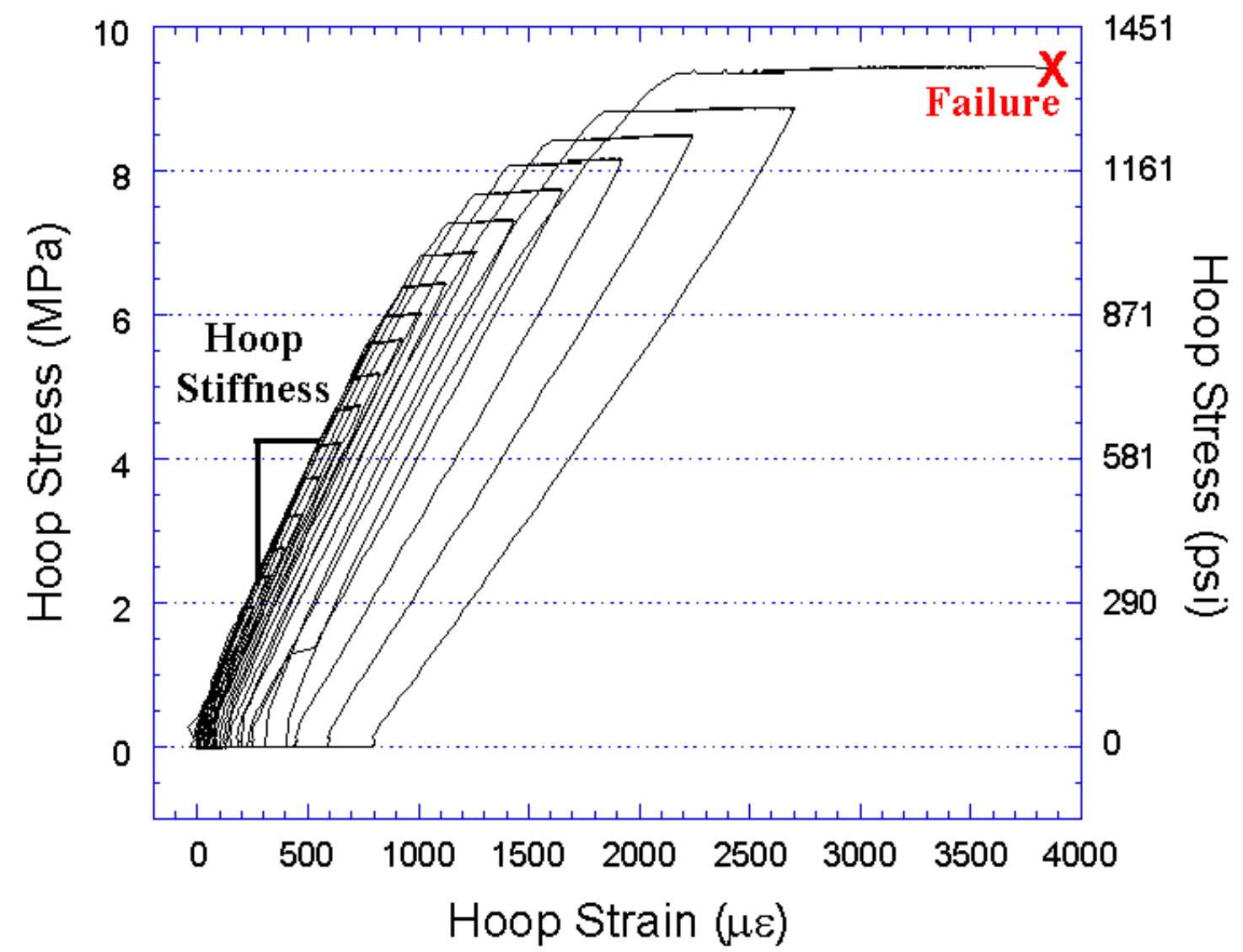

Figure 5.7 Hoop stiffness decreased due to damage during repeated load cycles.

The hoop stiffness values at each quadrant from hoop stress-strain curves during the range of applied pressure were determined. Stiffness values decreased with increasing hoop stress (Figures 5.8). A power fit of the data for each quadrant was the best curve. The posterior quadrant has the lowest stiffness for almost the entire hoop stress range (Figure 5.9). The mean values of hoop stiffness (Table 5.4) for the entire range of hoop stress among the quadrants are similar to the mean initial hoop stiffness determined from only the initial pressure (Table 5.2). 


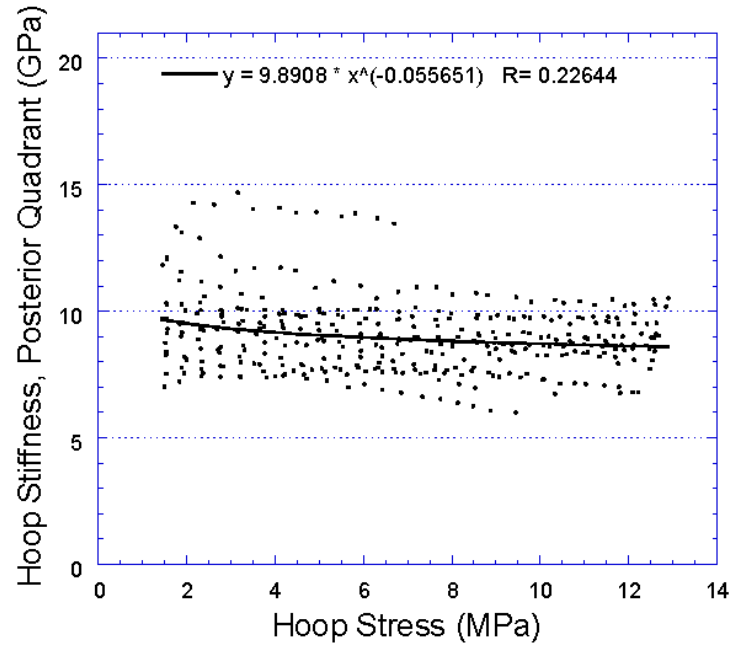

(A)

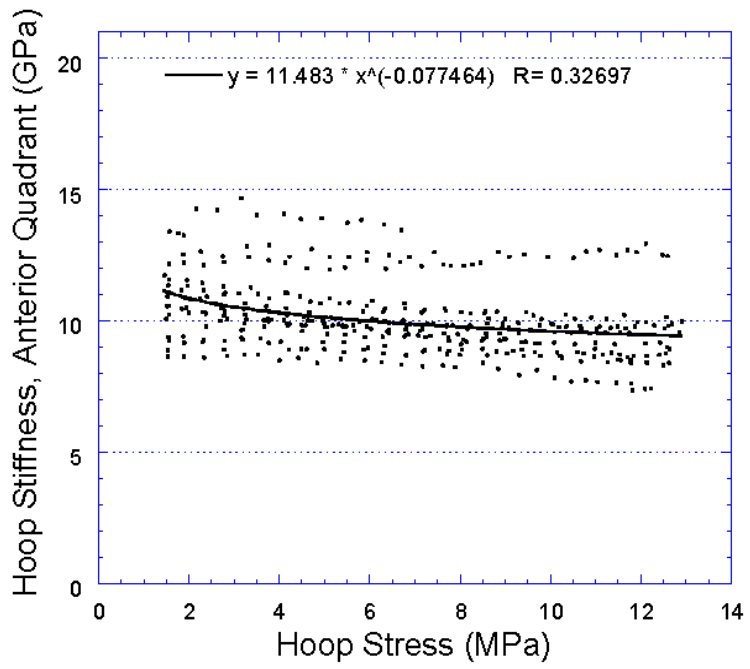

(C)

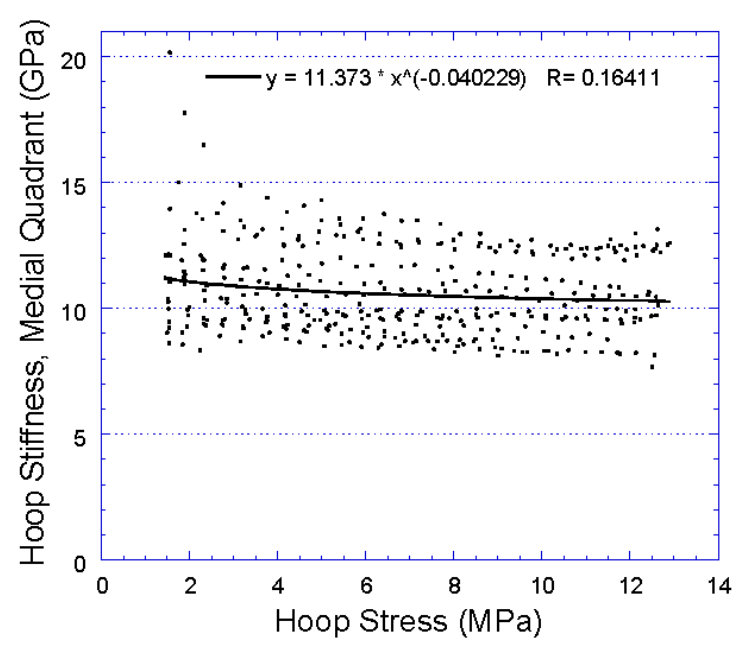

(B)

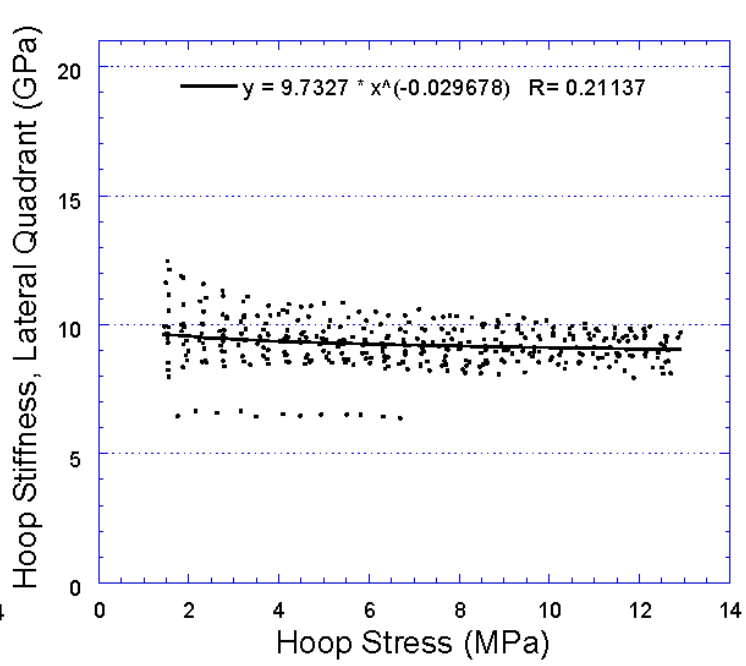

(D)

Figure 5.8 The hoop stiffness for all specimens at each quadrant and a power fit: (A) posterior, (B) medial, (C) anterior and (D) lateral quadrants. 


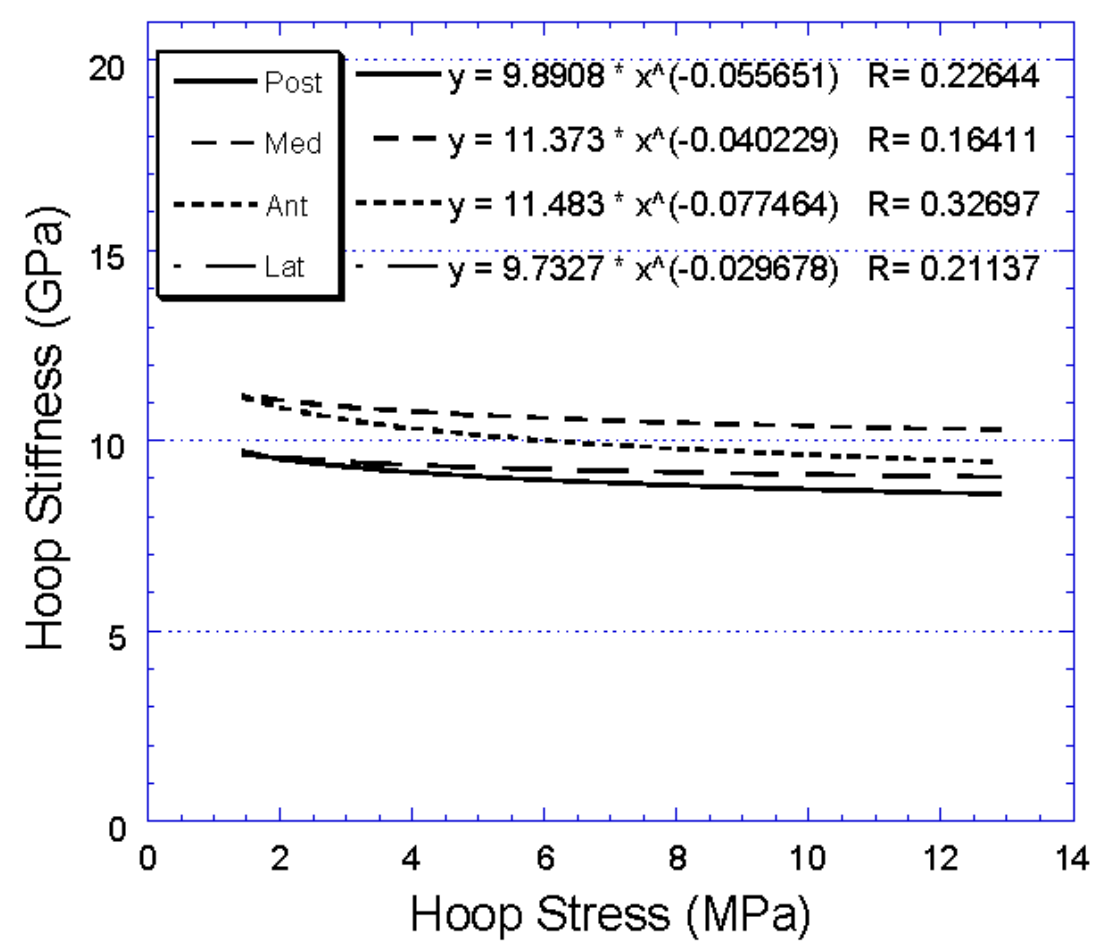

Figure 5.9 Power fits of the hoop stiffness for all four quadrants throughout the hoop stress range.

Table 5.4 The mean hoop stiffness values from each quadrant determined from the entire hoop stress range.

\begin{tabular}{|c|c|c|}
\hline Quadrant & $\begin{array}{c}\text { Hoop Stiffness } \\
\text { Mean } \pm \text { St.D. (GPa) }\end{array}$ & $\begin{array}{c}\text { Max, Min } \\
\text { (GPa) }\end{array}$ \\
\hline Posterior & $9.07 \pm 1.48$ & $14.70,5.99$ \\
\hline Medial & $10.72 \pm 1.74$ & $20.16,7.67$ \\
\hline Anterior & $10.10 \pm 1.41$ & $14.70,7.37$ \\
\hline Lateral & $9.28 \pm 0.91$ & $12.48,6.38$ \\
\hline Average & $9.79 \pm 1.56$ & $20.16,5.99$ \\
\hline
\end{tabular}

\subsection{Strain Rate During Pressurization}

Linear regression of the hoop strain against time was used to model the behavior during the loading phase while the pressure was applied at a constant rate. The slope of this line was the strain rate (Figure 5.10). The internal pressure range for all the specimens was between 0.345 and $3.06 \mathrm{MPa}$ (50 and $444 \mathrm{psi}$ ) and throughout the pressure range the strain rate increased during pressurization. The lowest strain rate 
during the loading phase was $170.37 \mu \varepsilon / \mathrm{s}$ (Test 18) and the highest strain rate was 2257.3 $\mu \varepsilon / \mathrm{s}$ (Test 12). The difference in strain rate of the bone specimens was not expected to affect the stiffness based on previous research.

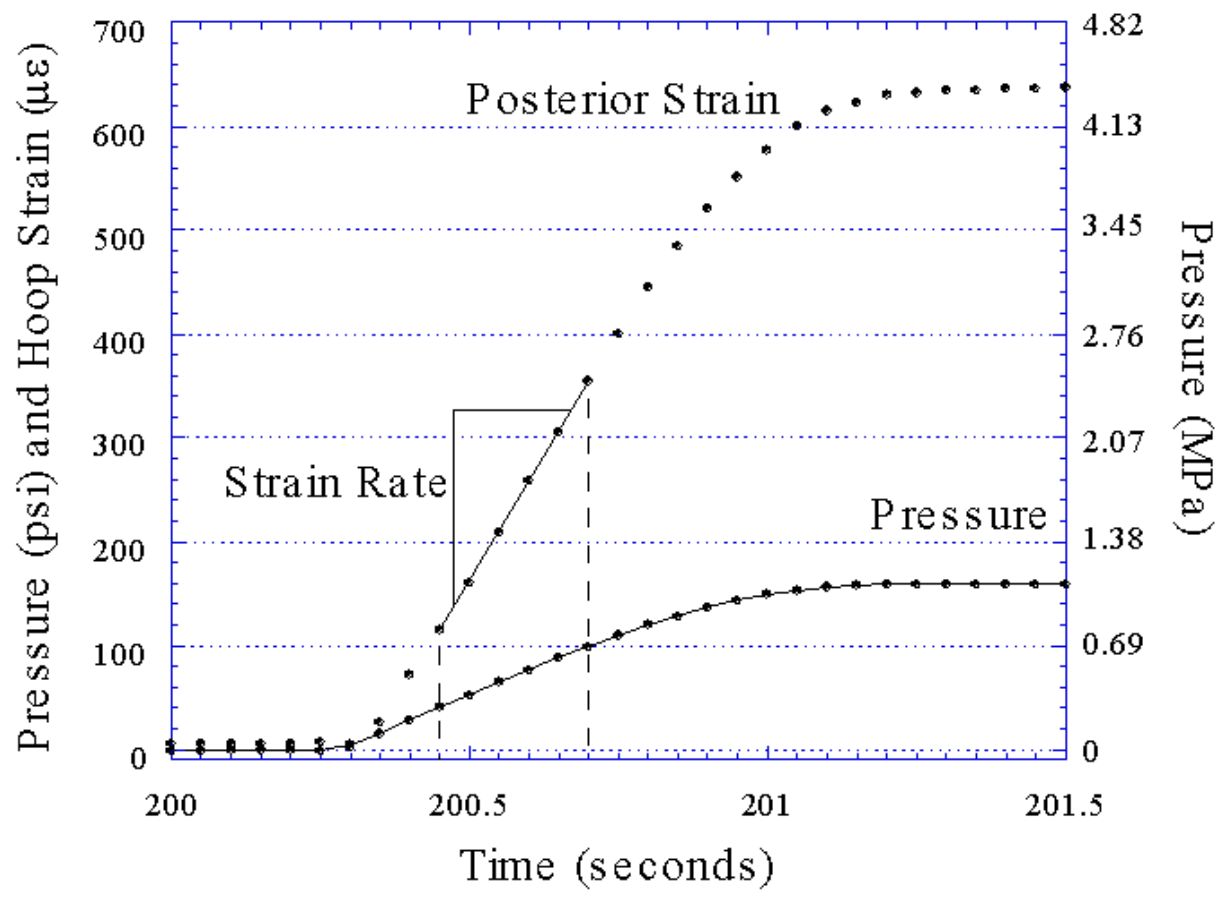

Figure 5.10 The strain rate, $958.63 \mu \varepsilon / \mathrm{s}$, was determined as the slope of the linear portion, between 200.45 and 200.65 seconds, where the load was applied at a constant rate, $1.64 \mathrm{MPa} / \mathrm{s}$ (238.63 $\mathrm{psi} / \mathrm{s})$ (region bounded by the dotted lines) (Test 8d).

The strain rate measured in this study $\left(0.00017\right.$ to $\left.0.0023 \mathrm{~s}^{-1}\right)$ is slower than physiological strain rates determined for the human tibia shaft under normal activity, 0.002 to $0.01 \mathrm{~s}^{-1}$ and for traumatic fractures, 0.1 to $1 \mathrm{~s}^{-1}$ (Lanyon et al., 1975). The effects of the strain rate on mechanical properties of bone in tension (Crowninshield and Pope, 1974) (Table 5.2) and compression (McElhaney, 1966) have been studied. At a given strain rate, bone is strongest in compression, less strong in longitudinal tension, and weakest in tension in the transverse direction. The modulus of elasticity for bone in tension was not greatly affected by strain rate, however, for compression, as the strain rate increased, the modulus of elasticity increased. The low strain rate shear failures result from a distortion of the lamellar substructure, resulting in failure along the weakest planes. High rate failures, however, appeared to follow cement lines. Wright and Hayes 
(1976) tested longitudinally oriented bovine bone specimens in uniaxial tension tests. Strength, stiffness and energy absorption capacity were significantly dependent on strain rate over a large range, $5.3 \mathrm{E} 10^{-4}$ to $237 \mathrm{~s}^{-1}$. Melnis and Knets (1982) conducted tensile tests on human tibia bone. As the strain rate increased from $10^{-5}$ to $1 \mathrm{~s}^{-1}$, the ultimate stress increased, however, the elastic modulus remained virtually constant. Currey (1988) studied the effects of strain rate and mineralization on the mechanical properties of bone. The elastic modulus was found to be barely strain-rate dependent. They suggest that a specimen with a high stress rate will have less time to accumulate damage and will have a higher ultimate strain.

\subsection{Static Modulus}

The static modulus was determined for each specimen (Table 5.5). The maximum hoop strain during the constant load phase was used from each stress level (Figure 5.11A). The hoop stress versus maximum hoop strain was plotted (Figure 5.11B) and has an initial linear region followed by a nonlinear region. A linear regression of the hoop stress against maximum hoop strain was used to model the initial behavior and determine the static modulus.

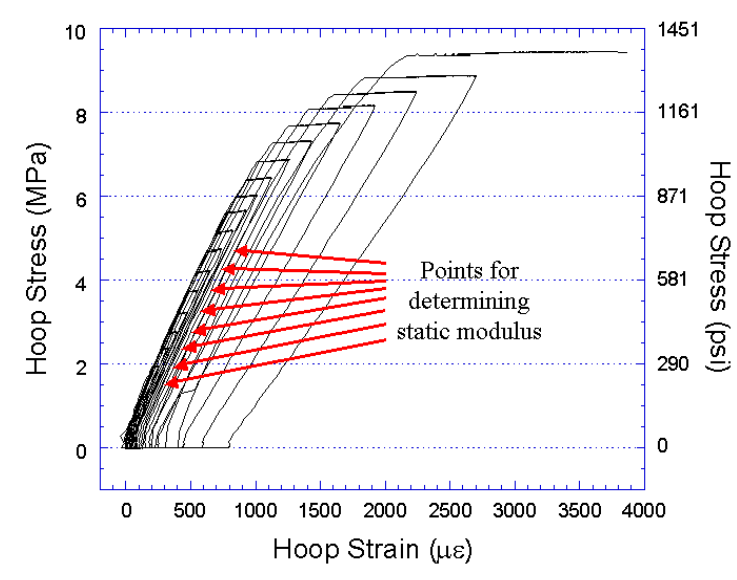

(A)

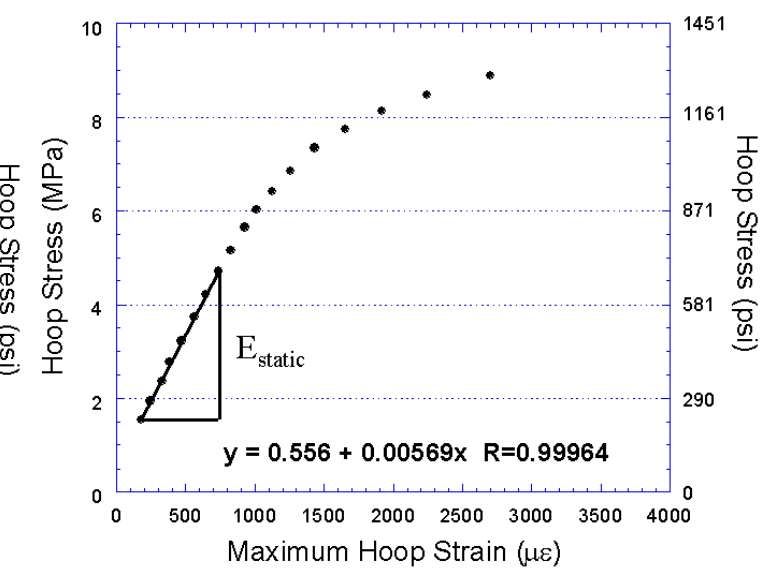

(B)

Figure 5.11 (A) A hoop stress-strain curve for a single specimen showing the maximum hoop strain during at each hoop stress level. (B) The static modulus is determined from the slope of the curve of the initial linear region, $\sigma_{\mathrm{H}}<4.71 \mathrm{MPa}(684.09 \mathrm{psi})$, of the hoop stress versus maximum hoop strain (posterior, Test 8). The static modulus is $5.69 \mathrm{GPa}$ (0.83E6 psi). 
Table 5.5 The static modulus for each of the 15 specimens at each quadrant.

\begin{tabular}{|c|c|c|c|c|}
\hline $\begin{array}{c}\text { Specimen } \\
\text { Test \# }\end{array}$ & $\begin{array}{c}\text { Posterior } \\
(\mathbf{G P a})\end{array}$ & $\begin{array}{c}\text { Medial } \\
(\mathbf{G P a})\end{array}$ & $\begin{array}{c}\text { Anterior } \\
(\mathbf{G P a})\end{array}$ & $\begin{array}{c}\text { Lateral } \\
(\mathbf{G P a})\end{array}$ \\
\hline 4 & 6.08 & 9.73 & 12.69 & 5.97 \\
\hline 5 & 7.43 & 7.99 & 7.97 & 6.98 \\
\hline 6 & 5.98 & 8.21 & 8.04 & 6.95 \\
\hline 7 & 6.32 & 7.59 & 10.75 & 6.90 \\
\hline 8 & 5.69 & 7.08 & 7.44 & 6.42 \\
\hline 9 & 7.59 & 7.25 & 8.38 & 6.33 \\
\hline 10 & 8.48 & 9.49 & 6.78 & 6.81 \\
\hline 11 & 6.42 & 6.51 & 8.31 & 7.11 \\
\hline 12 & 5.38 & 7.69 & 5.99 & 8.38 \\
\hline 13 & 7.61 & 10.11 & 7.23 & 7.69 \\
\hline 14 & 6.97 & 8.86 & 11.51 & 8.39 \\
\hline 15 & 7.04 & 11.43 & 8.38 & 7.18 \\
\hline 16 & 7.24 & 6.41 & 7.56 & 7.55 \\
\hline 17 & 7.11 & 8.61 & 7.42 & 7.42 \\
\hline 18 & 8.12 & 10.52 & 8.90 & 7.33 \\
\hline Mean \pm St.D. & $6.90 \pm 0.90$ & $8.50 \pm 1.50$ & $8.49 \pm 1.82$ & $7.16 \pm 0.68$ \\
\hline Mean of all & & \multicolumn{3}{l}{} \\
Quads \pm St.D. & & $7.76 \pm 1.48$ & \\
\hline
\end{tabular}

The ratio of the mean static modulus, $7.76 \mathrm{GPa}$, and the mean instantaneous stiffness, $10.52 \mathrm{GPa}$, was 0.74 . This ratio was an indication of the deviation from linear elastic behavior, based on the instantaneous stiffness, caused by the viscoelastic effects, as seen by the static modulus (Fondrk et al., 1988).

\subsection{Creep Strain}

The creep strain, increasing deformation under a constant load, was calculated as the difference between the hoop strain measured at the beginning and at the end of the 1minute constant pressure phase (Figure 5.12A). As the stress levels increased, the amount of creep strain increased as well (Figure 5.12B). 


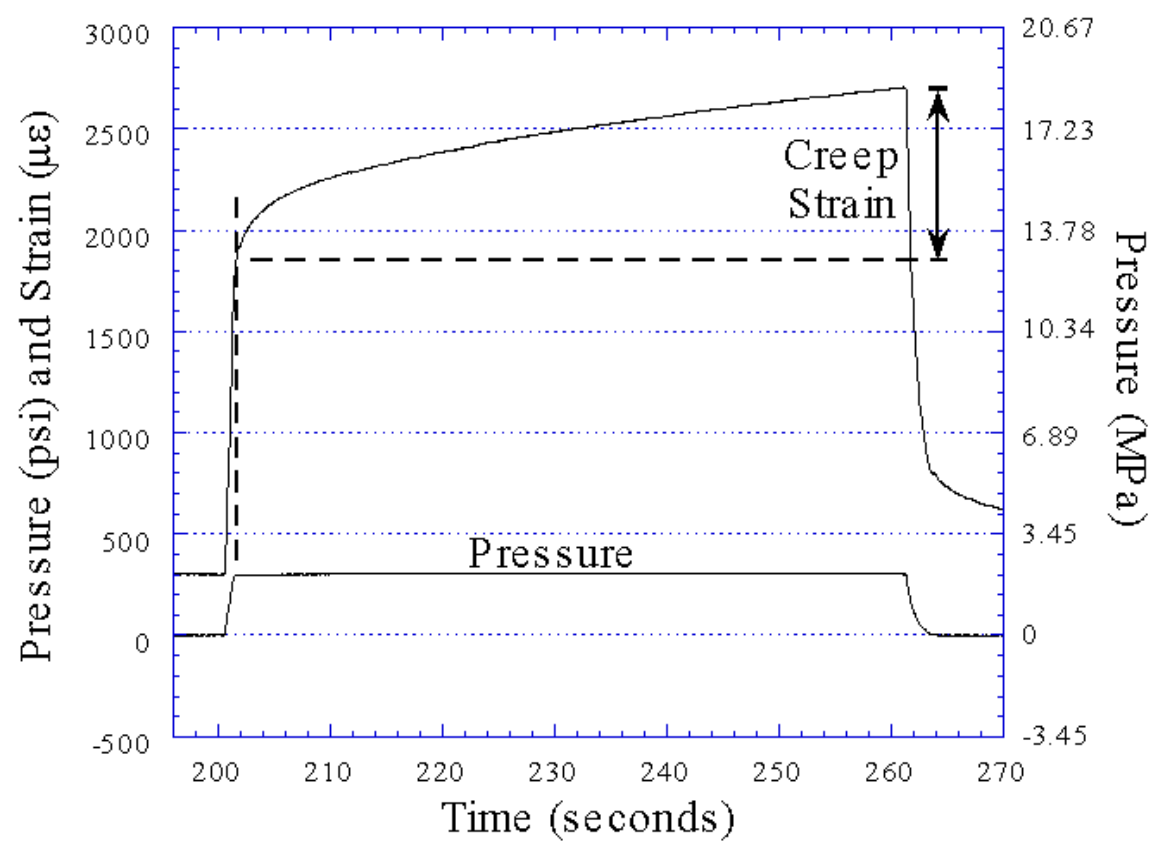

(A)

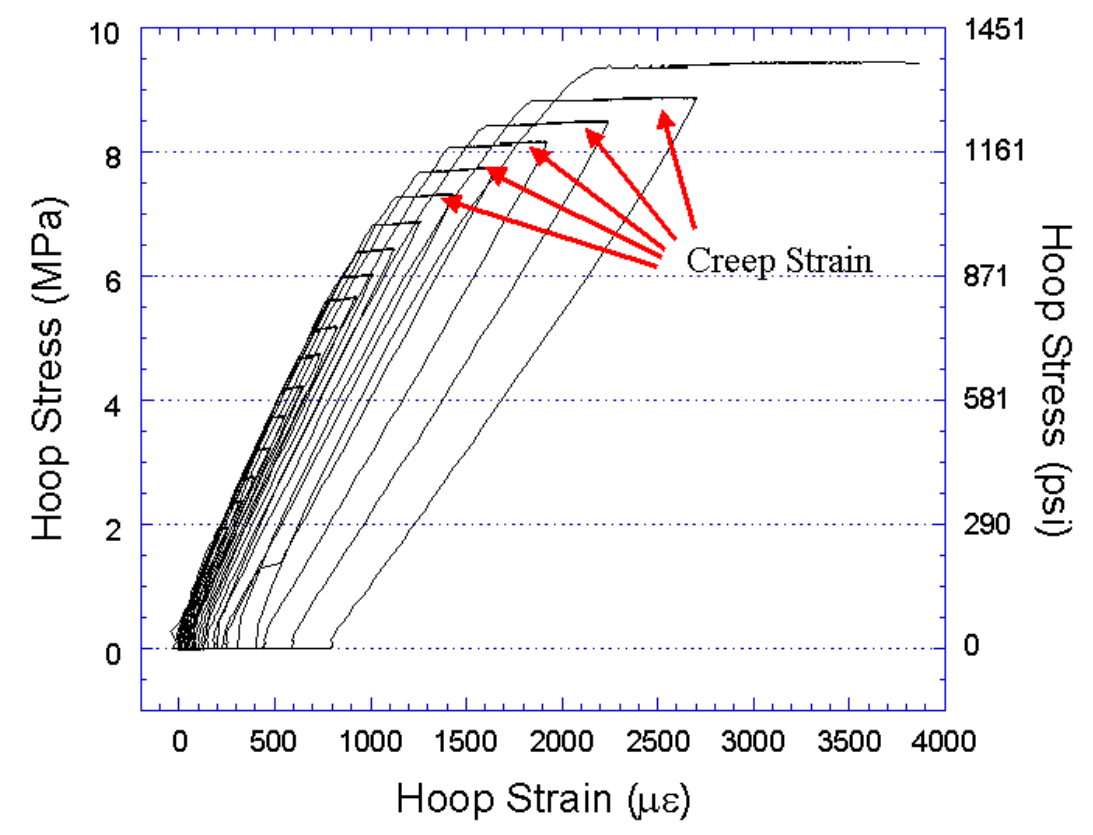

(B)

Figure 5.12 (A) Creep strain measured from the hoop strain during the 1-minute of constant pressure (Test $8 \mathrm{i}$ ). The creep strain, $853 \mu \varepsilon$, was calculated as the difference between the initial strain, $1849.3 \mu \varepsilon$, and the final strain after 1 minute, $2702.3 \mu \varepsilon$. (B) Creep strain increases with increasing hoop stress. 
The creep strain for all specimens was plotted against hoop stress (Figure 5.13). The initial internal pressure of $0.345 \mathrm{MPa}$ (50 psi) caused a hoop stress of approximately 1.52 $\mathrm{MPa}$ (220 psi), which is the first data point for all specimens. The solenoid valve prevented the internal pressure from exceeding approximately $3.06 \mathrm{MPa}$ (444 psi) or a hoop stress value of approximately $13.1 \mathrm{MPa}$ (1900 psi), which is the final hoop stress. Some specimens did not fail before the final pressure level of $3.06 \mathrm{MPa}$ (444 psi) was reached. For the specimens that did fail below 3.06 MPa (444 psi), the final point used for creep strain data was the last load phase in which the specimen survived the entire one minute of constant pressure. The creep strain of the 1-minute phase when the specimen fractured was not included since the full minute of loading was not completed.

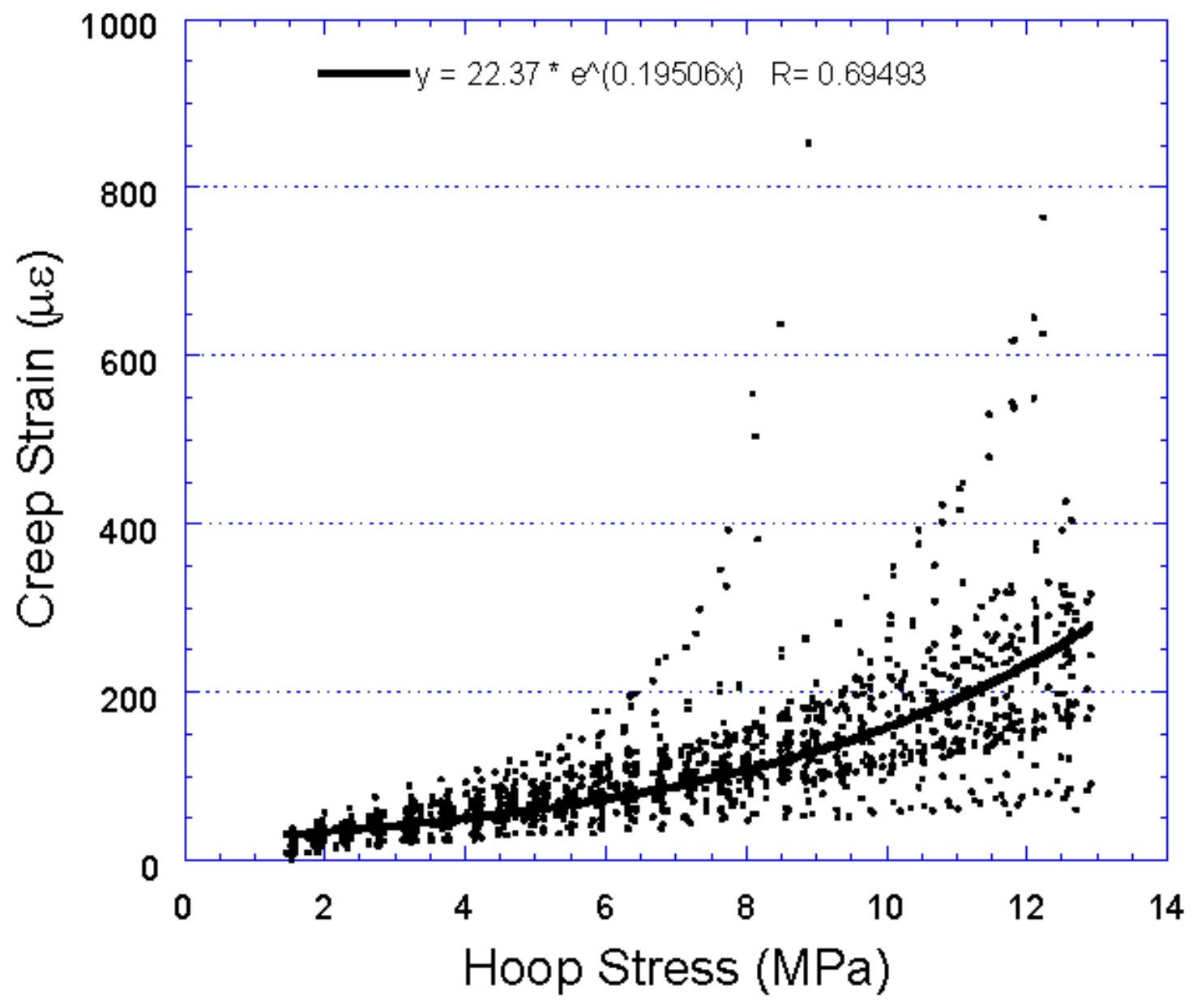

Figure 5.13 Creep strain versus hoop stress for all 15 specimens from all quadrants. 
To more accurately compare strain measurements from different donors accounting for differences in material properties among specimens, the hoop stress was normalized using hoop stiffness values determined at every load level during the tests (Figure 5.14) (Caler and Carter, 1989; Mauch et al., 1992; Bowman et al., 1998; 1999). A significant relationship exists between hoop creep strain and normalized hoop stress $(p<0.0001)$. Various fits of the data were made and the exponential fit provided the best curve and was used to model the combined creep strain data for all specimens (Table 5.6).

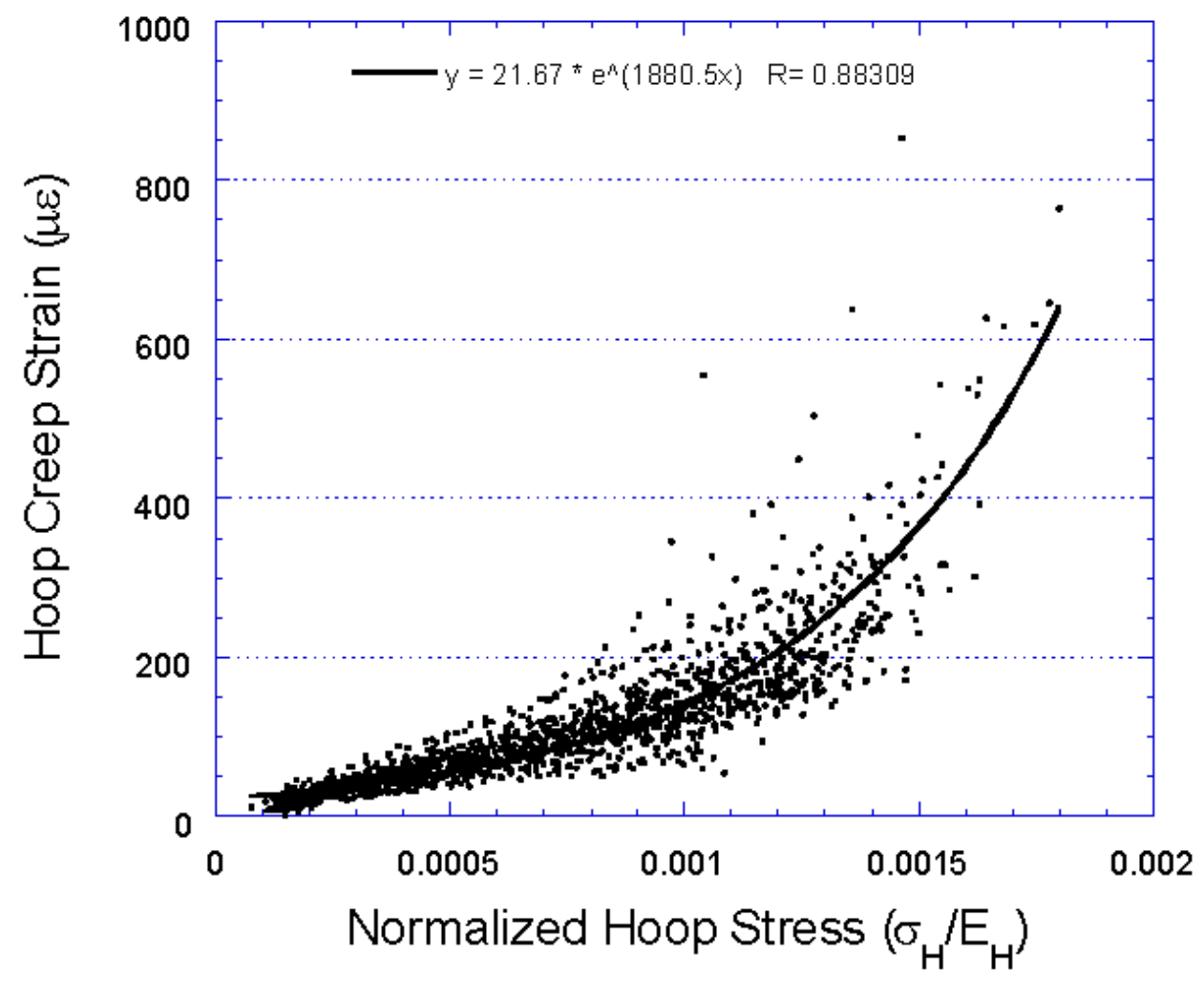

Figure 5.14 Creep strain variation with hoop stress normalized with hoop stiffness from each stress level. The exponential fit provided the best curve.

Table 5.6 Equations of various fits for hoop creep strain versus normalized hoop stress.

\begin{tabular}{|c|l|c|}
\multicolumn{1}{c}{ Fit } & \multicolumn{1}{c}{ Equation } & \multicolumn{1}{c|}{ R } \\
\hline Exponential & $\mathrm{y}=21.67 * \mathrm{e}^{\wedge}(1880.5 \mathrm{x})$ & 0.88 \\
\hline Polynomial & $\mathrm{y}=44.70-63045 \mathrm{x}+1.7 \mathrm{E} 8 \mathrm{x}^{2}$ & 0.87 \\
\hline Power & $\mathrm{y}=4.38 \mathrm{E} 5 * \mathrm{x}^{\wedge}(1.1561)$ & 0.84 \\
\hline Linear & $\mathrm{y}=-35.33+2.033 \mathrm{E} 5 \mathrm{x}$ & 0.83 \\
\hline Logarithmic & $\mathrm{y}=919.49+251.23 \log (\mathrm{x})$ & 0.73 \\
\hline
\end{tabular}


The plots of the creep strain and normalized hoop stress for each individual quadrant were examined to observe differences in behavior (Figure 5.15). The fit model platform in JMP was used to apply the standard least squares for general linear models. There were significant differences among the curve fits for the different quadrants, however, if the same equation was used to fit the responses for all quadrants, the loss in predictive ability was less than $2 \%\left(\mathrm{R}^{2}=0.767\right.$ versus $\left.\mathrm{R}^{2}=0.782\right)$. Based on this statistical analysis, the measured strain from all four quadrants was analyzed as one group (Figure $5.14)$.

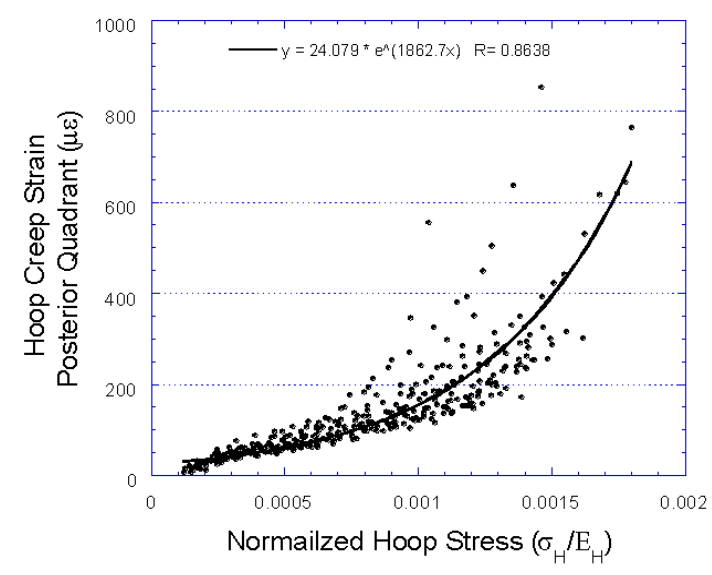

(A)

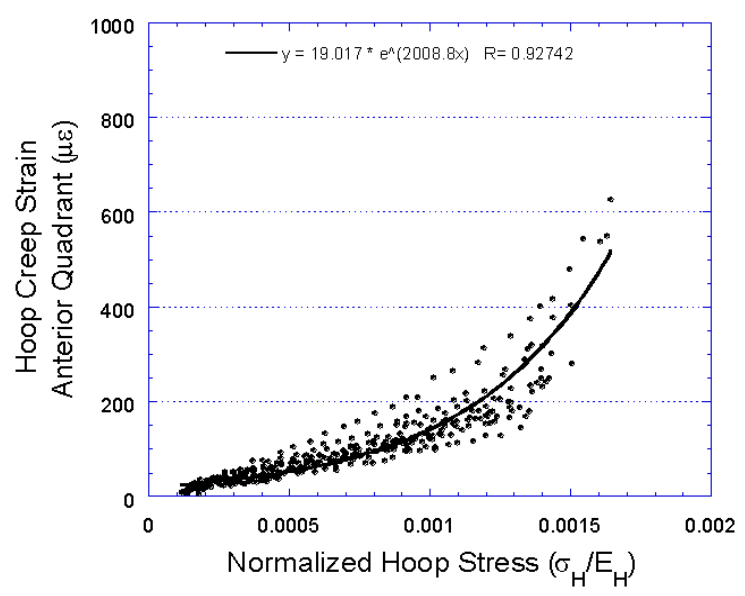

(C)

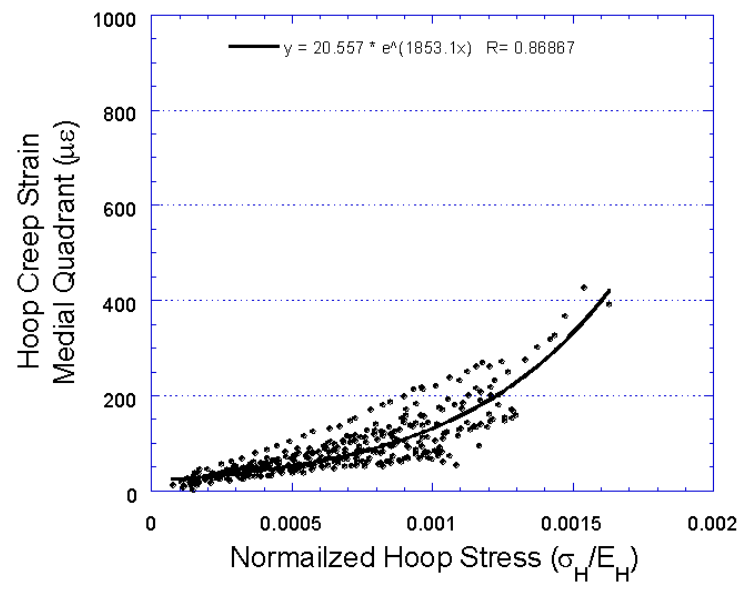

(B)

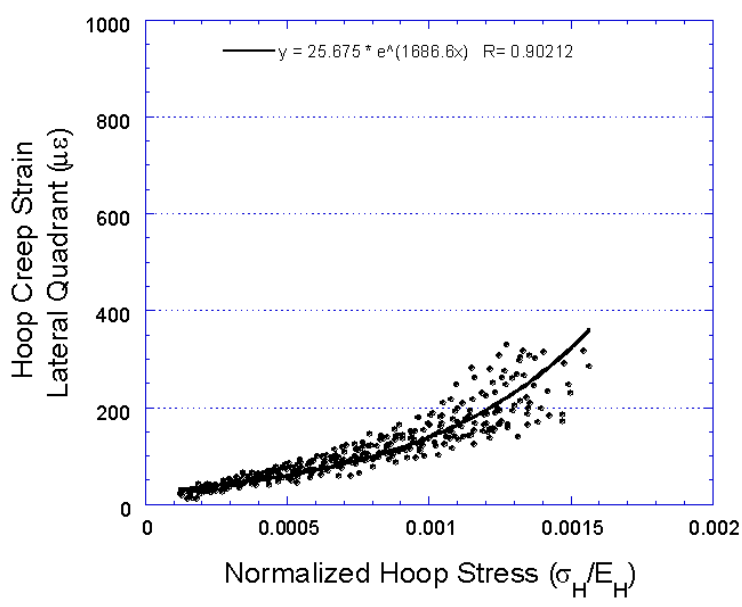

(D)

Figure 5.15 Creep strain variation with normalized hoop stress for each quadrant, posterior (A), medial (B), anterior (C) and lateral (D). An exponential curve was used to fit the data in each plot. 


\subsubsection{Creep Strain Threshold}

A method was needed to determine the point of behavioral change in the exponential fit of the creep strain of all the quadrants (Figure 5.14). The overall shape of the data was similar to a stress-strain curve for a uniaxial tension test of a material. For materials where the yield strength is not characterized by a horizontal portion of the stress-strain curve, the yield strength is defined by an offset method. This method involves first defining the amount of strain as the offset, $0.2 \%$ is typically used. Next, the slope of the linear portion of the stress-strain curve is determined. The offset line is constructed with this slope and passes through the offset strain. The stress corresponding to the intersection of this line and the stress-strain curve is defined as the yield strength (Beer and Johnston, 1991; Byars et al., 1983; Boresi et al., 1993).

A method similar to determining the yield strength of materials was followed to determine a point of behavioral change in the exponential fit. The specimens exhibited linear behavior during the low stress levels (Figure 5.16). Creep strain data below a normalized stress level of $0.0005\left(\sigma_{\mathrm{H}} / \mathrm{E}_{\mathrm{H}}<0.0005\right)$ was chosen for linear regression of the creep strain data against normalized hoop stress to model the initial behavior. The linearity of the creep data in this region was emphasized by the nearness of the $y$ intercept to zero (0.039) as well as the correlation coefficient $(\mathrm{R}=0.79)$ (solid line in Figure 5.16). The slope of the fit representing the linear behavior (slope=1.34E5) was used as the slope of the offset line.

The amount of offset was determined by the upper bound of the $95 \%$ confidence interval from the data of the initial behavior. A linear fit of the upper bound of the $95 \%$ confidence interval over the range of normalized hoop stress was used to determine the $y$ intercept of the offset line, $21.73 \mu \varepsilon$. This procedure for determining the offset line was chosen as a statistical method for defining an offset line for the initial behavior of the creep strain, creep rate and permanent strain data. 


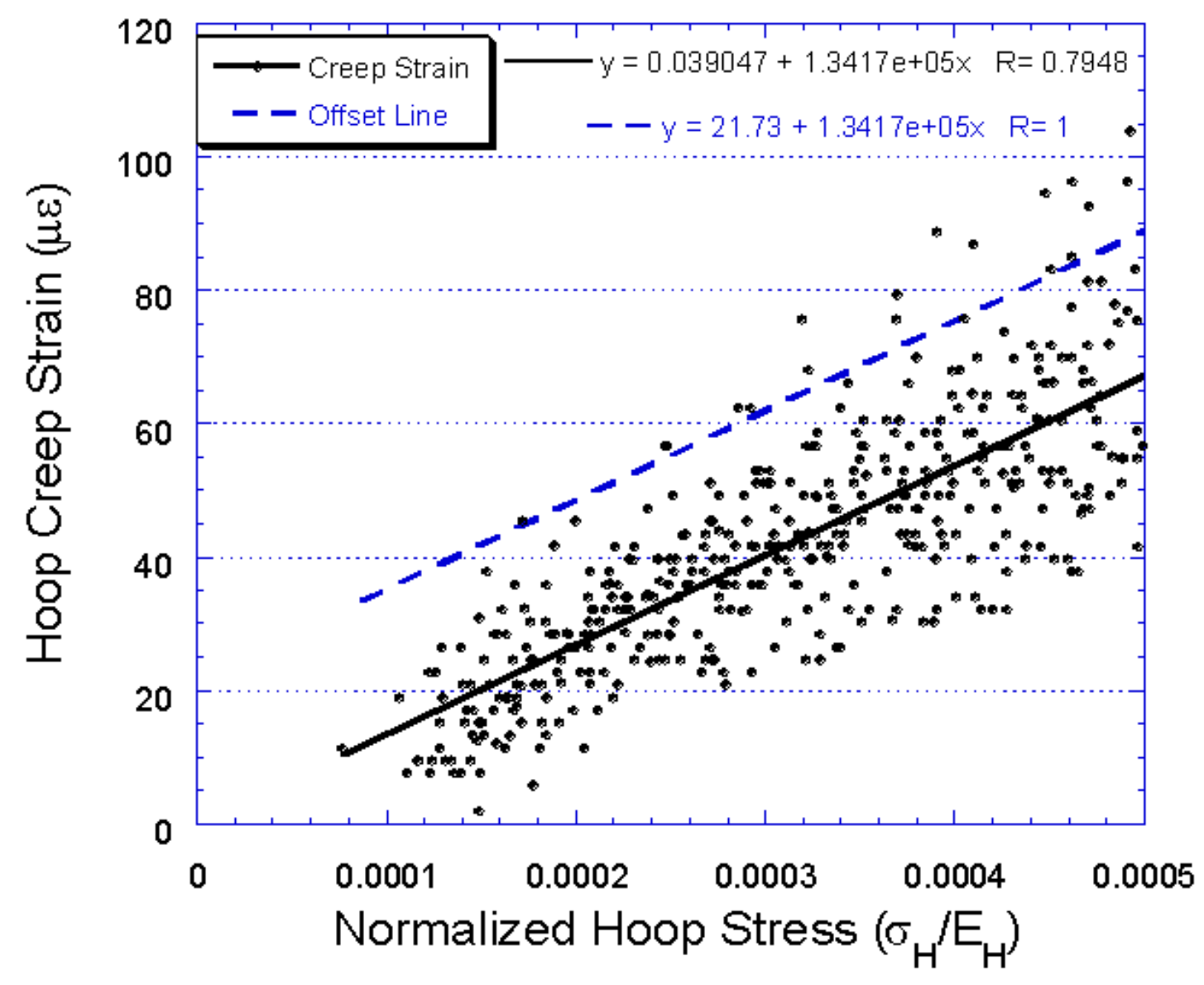

Figure 5.16 Creep strain measured during the normalized hoop stress levels that resulted in linear behavior $\left(\sigma_{\mathrm{H}} / \mathrm{E}_{\mathrm{H}}<0.0005\right)$. The dashed line was offset from the linear fit of the data represented by the solid line.

The intersection of this offset line and the exponential fit serves to define the point in which the creep strain data deviates from initial linear behavior and becomes nonlinear. The intersection of the linear offset and exponential fit indicates a threshold value of normalized hoop stress, 0.001088 , and creep strain, $167.6 \mu \varepsilon$, at which the creep strain begins to increase nonlinearly (Figure 5.17). 


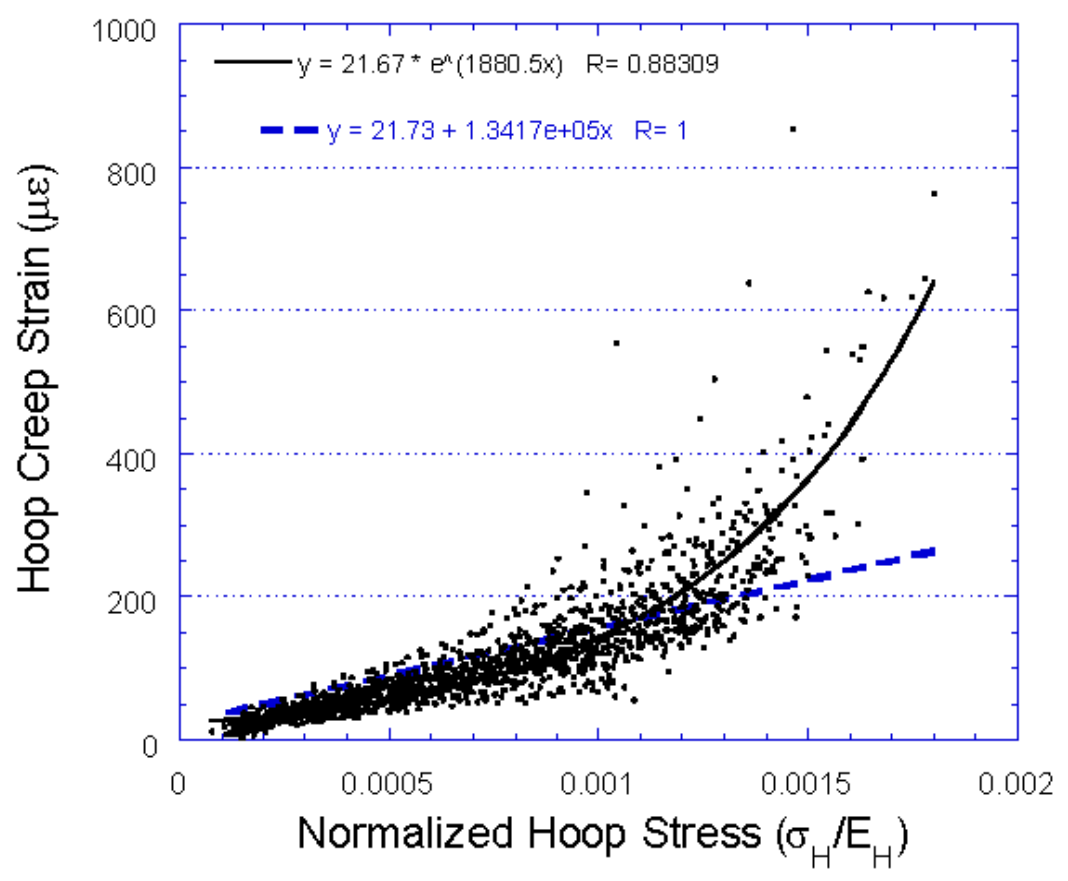

(A)

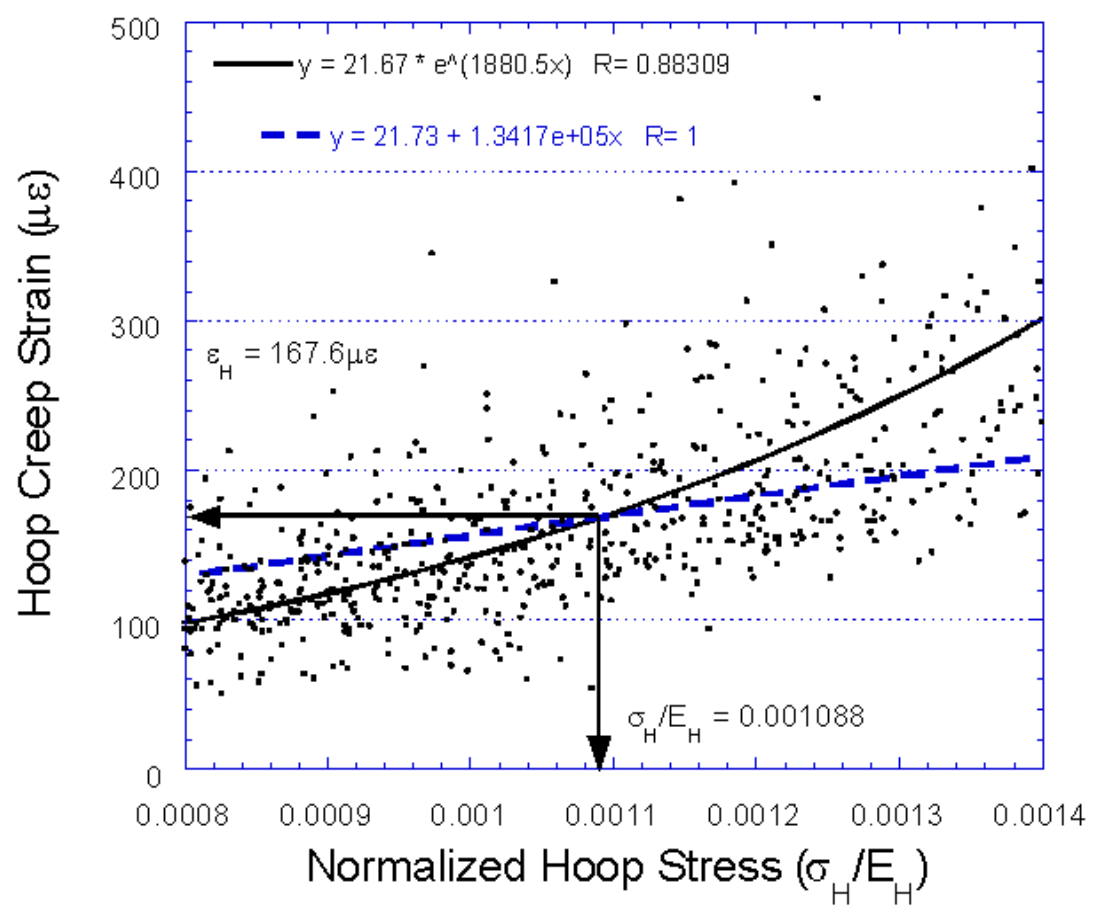

(B)

Figure 5.17 The threshold is the intersection between the exponential fit of the data and the offset line. 


\subsubsection{Multivariable Model of Creep Strain}

Stepwise regression analysis was used for scanning variables for including in a regression model. A multivariable model using the least squares procedure was used for estimating creep strain $(\mu \varepsilon)$ (Table 5.7). The control parameters examined were normalized hoop stress, location, gender, age, porosity, osteon area, osteon density, single osteon area, and initial stiffness. Those that were found suitable for a model were normalized hoop stress (nhs), location (L-P), porosity (Po), osteon density (On.Dn) and initial stiffness (In.St) (GPa).

Table 5.7 Multivariable model of creep strain.

\begin{tabular}{|c|c|c|}
\hline \multicolumn{3}{|c|}{ Creep strain $=202104.99 \mathrm{nhs}+3.120 \mathrm{Po}-2.145 \mathrm{In} . \mathrm{St}-7.191$ Location $(\mathrm{L}-\mathrm{P})+2.235$} \\
\hline On.Dn -84.880 & & $(5.5)$ \\
\hline nhs & $\mathrm{r}^{2}=0.688$ & $\mathrm{p}<0.0001$ \\
\hline Po & $\mathrm{r}^{2}=0.724$ & $\mathrm{p}<0.0001$ \\
\hline In.St & $r^{2}=0.727$ & $\mathrm{p}=0.0018$ \\
\hline Location (L-P) & $\mathrm{r}^{2}=0.730$ & $\mathrm{p}=0.0036$ \\
\hline On.Dn & $\mathrm{r}^{2}=0.731$ & $\mathrm{p}=0.0121$ \\
\hline Model & $\mathrm{r}^{2}=0.732$ & $\mathrm{p}<0.0001$ \\
\hline
\end{tabular}

The model predicts that creep strain will have a positive relationship with normalized hoop stress, porosity and osteon density and an inverse relationship with initial stiffness and location. Porosity explains a portion of the variability, 3.6\%, second only to normalized hoop stress. Initial stiffness explains only an additional $0.3 \%$ of the variability after porosity is included in the model. 


\subsection{Creep Rate}

The creep rate, the rate at which creep strain occurs, was determined for each constant pressure phase by linear regression analysis of the creep strain data during the last 40 seconds of the 1-minute load phase (Fondrk et al., 1988) (Figure 5.18).

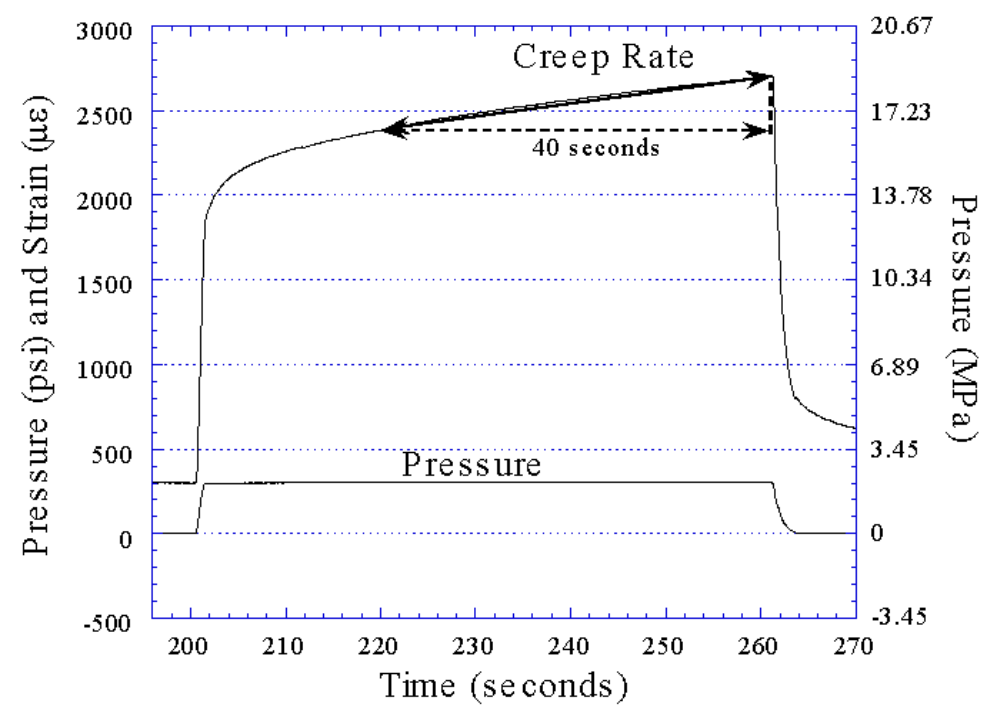

(A)

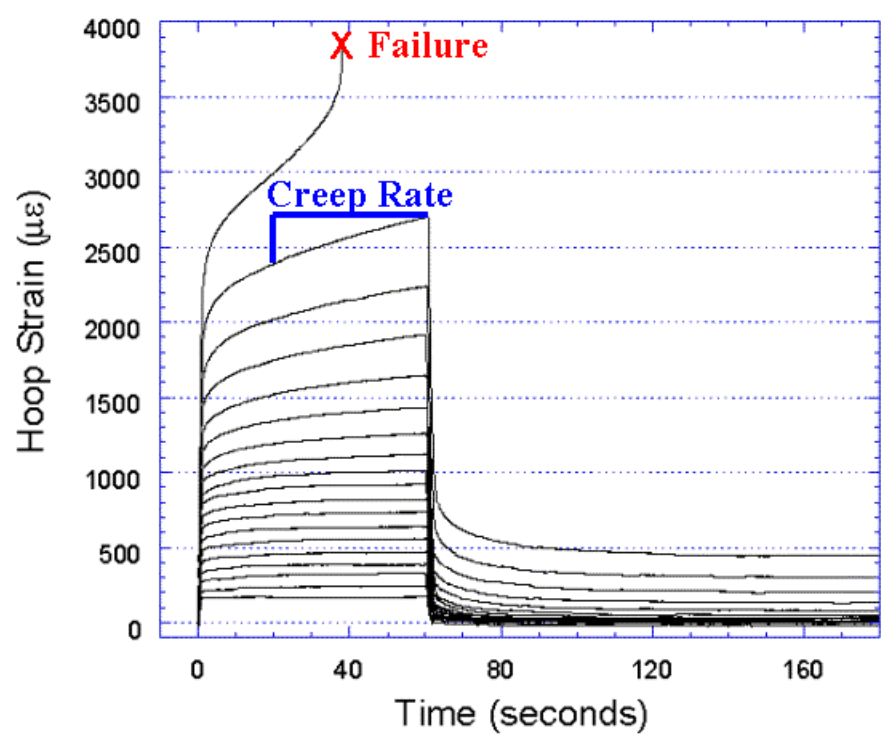

(B)

Figure 5.18 (A) The creep rate $(7.5 \mu \varepsilon / \mathrm{s})$ during the last 40 seconds of the 1-minute load phase (pressure $=2.08 \mathrm{MPa}(301.68 \mathrm{psi})$ ) (Test $8 \mathrm{i})$. (B) As the hoop stress levels increase, the creep rate also increases. 
For the specimens that did fail during testing, the final load-phase used for the creep rate was the last load-phase in which the specimen survived the entire one minute of constant pressure. The creep rate during the 1-minute phase when the specimen fractured was not included since the full minute of loading was not completed. The stress levels for the creep rate analysis were normalized as described above (Figure 5.19). An exponential curve provided the best fit of the data and was used to model the combined creep rate data for all specimens (Table 5.8). The plots of the creep strain versus normalized hoop stress for each individual quadrant were examined to observe differences in behavior. The fit model platform in JMP was used to apply the standard least squares for general linear models. There were significant differences among the curve fits for the different quadrants, however, if the same equation was used to fit the responses for all quadrants, the loss in predictive ability was less than $2 \%\left(\mathrm{R}^{2}=0.624\right.$ versus $\left.\mathrm{R}^{2}=0.640\right)$. Based on this statistical analysis, the creep rate from all four quadrants was analyzed as one group (Figure 5.19). A significant relationship was found between creep rate and normalized hoop stress $(\mathrm{p}<0.0001)$.

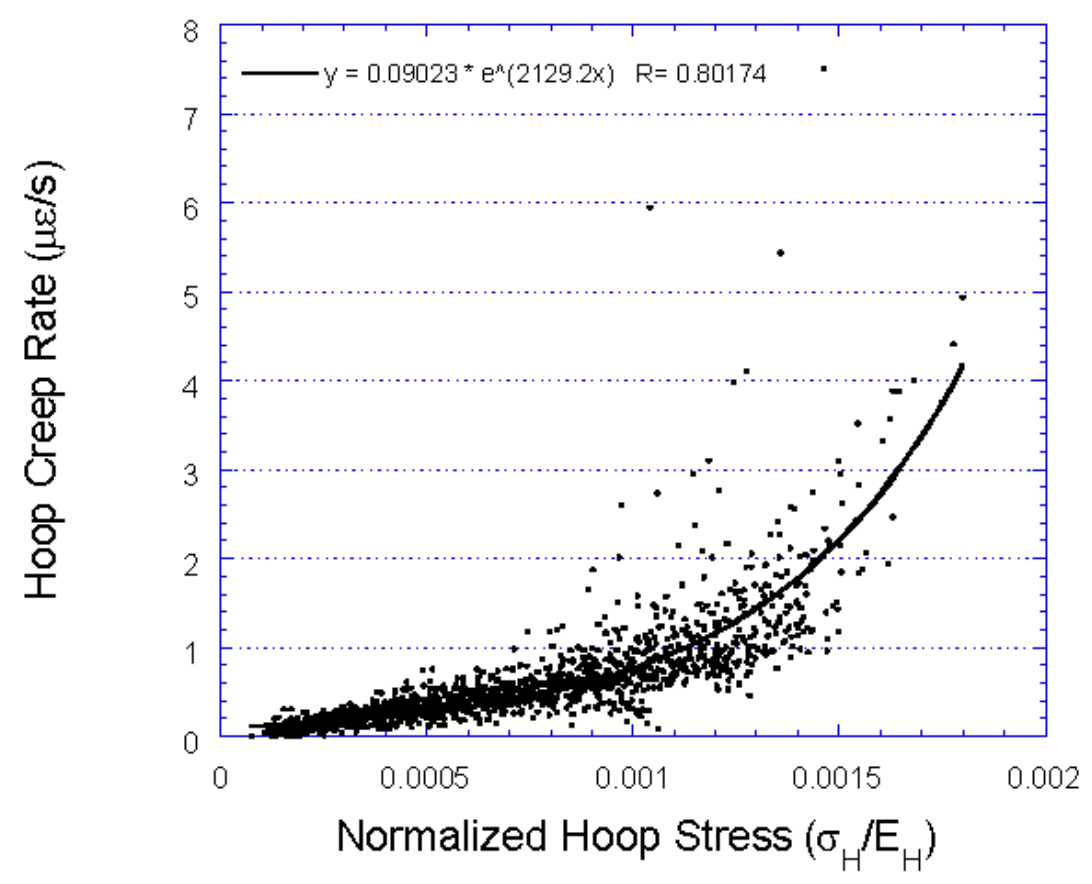

Figure 5.19 Creep rate for all 15 specimens from all quadrants for all hoop stress levels normalized with hoop stiffness, $\mathrm{p}<0.0001$. 
Table 5.8 Equations of various fits for hoop creep rate versus normalized hoop stress.

\begin{tabular}{|c|l|c|}
\multicolumn{1}{c}{ Fit } & \multicolumn{1}{c}{ Equation } & \multicolumn{1}{c|}{ R } \\
\hline Exponential & $\mathrm{y}=0.090 * \mathrm{e}^{\wedge}(2129.2 \mathrm{x})$ & 0.80 \\
\hline Polynomial & $\mathrm{y}=0.305-699.94 \mathrm{x}+1.2 \mathrm{E} 6 \mathrm{x}^{2}$ & 0.77 \\
\hline Power & $\mathrm{y}=8790.2 * \mathrm{x}^{\wedge}(1.3452)$ & 0.75 \\
\hline Linear & $\mathrm{y}=-0.286+1251 \mathrm{x}$ & 0.73 \\
\hline Logarithmic & $\mathrm{y}=5.585+1.5462 \log (\mathrm{x})$ & 0.64 \\
\hline
\end{tabular}

\subsubsection{Creep Rate Threshold}

The specimens exhibited linear creep rate behavior during low stress levels (Figure 5.19). Creep rate data below a normalized stress level of $0.0005\left(\sigma_{\mathrm{H}} / \mathrm{E}_{\mathrm{H}}<0.0005\right)$ was chosen for linear regression to model the initial behavior (Figure 5.20). The linear behavior in this region is emphasized by the nearness of the y-intercept of the linear fit $(-0.04)$ being near zero and the correlation coefficient $(\mathrm{R}=0.71)$. The slope of the fit representing the linear behavior, (slope $=801.57$ ), was used to create a line, offset by the upper bound of the $95 \%$ confidence interval.

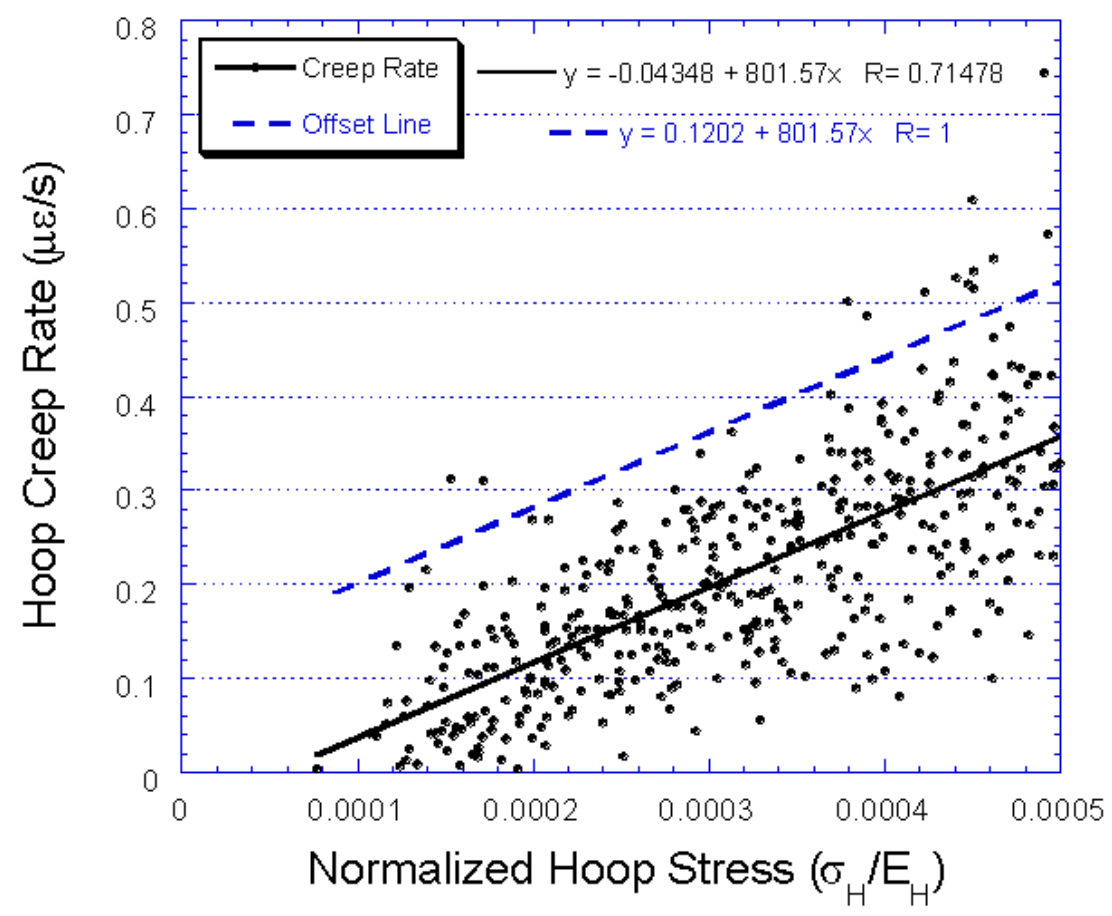

Figure 5.20 Creep rate measured during the normalized hoop stress levels that resulted in linear behavior $\left(\sigma_{H} / E_{H}<0.0005\right)$. The dashed line was offset from the linear fit of the data represented by the solid line. 
The intersection of the linear offset and exponential fit provides a threshold value of normalized hoop stress, 0.001148 , and creep rate, $1.04 \mu \varepsilon / \mathrm{s}$, at which the creep rate begins to increase nonlinearly (Figure 5.21).

(A)

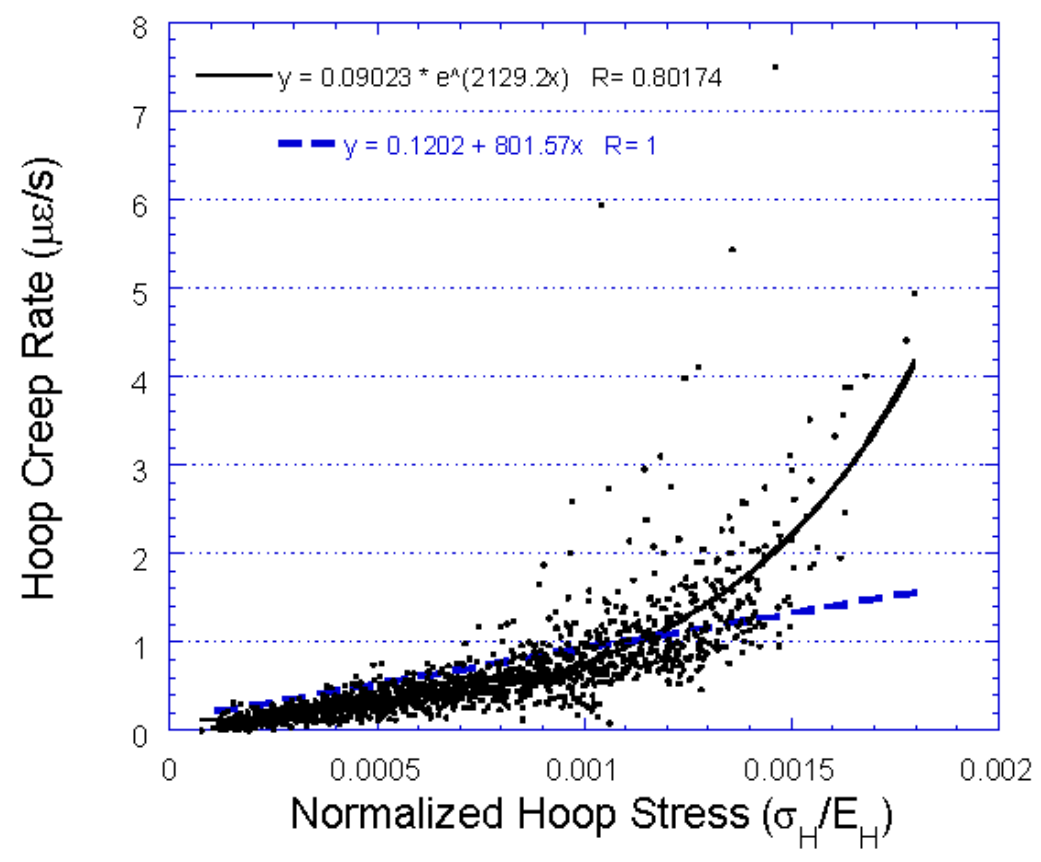

(B)

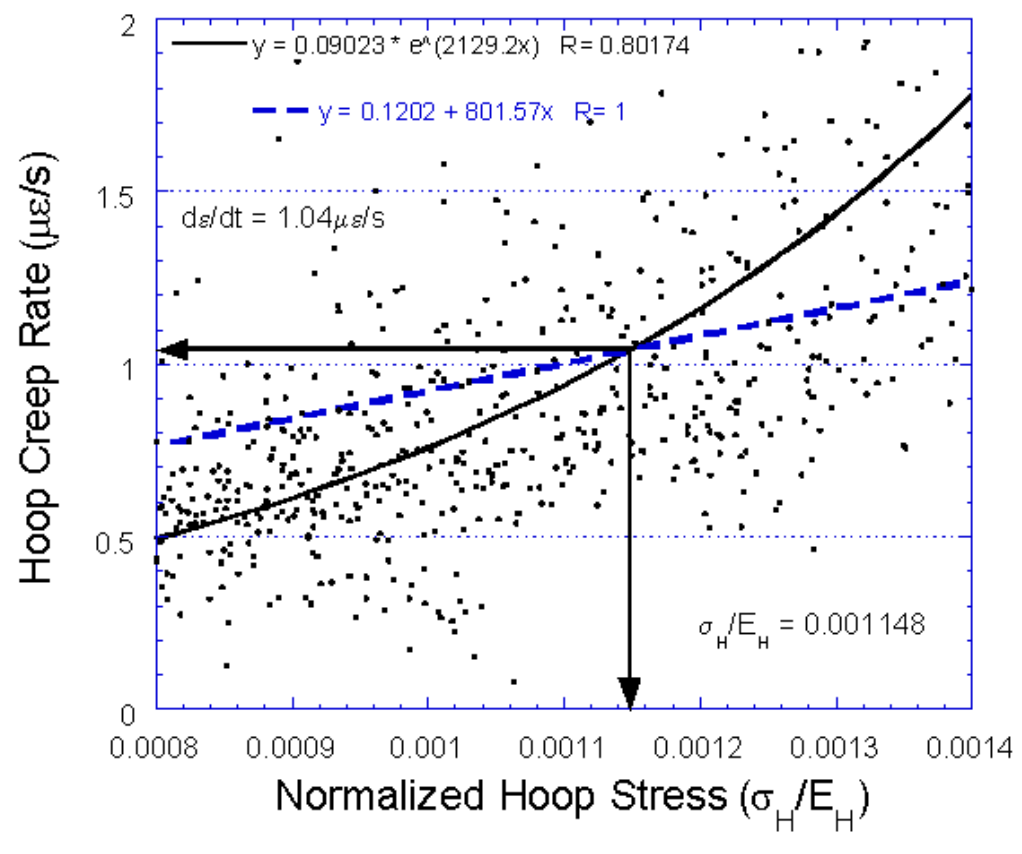

Figure 5.21 The creep rate threshold was the intersection between the exponential fit of all the data and the offset line. 


\subsubsection{Multivariable Model of Creep Rate}

Stepwise regression analysis was used for scanning variables for including in a regression model. A multivariable model using the least squares procedure was used for estimating creep rate $(\mu \varepsilon / \mathrm{s})$ (Table 5.9). The control parameters examined were normalized hoop stress, location, gender, age, porosity, osteon area, osteon density, single osteon area and initial stiffness. Those that were found suitable for the model were normalized hoop stress (nhs), porosity (Po), age and initial stiffness (In.St) (GPa).

Table 5.9 Multivariable model of creep rate.

\begin{tabular}{|c|c|c|}
\hline \multicolumn{3}{|c|}{ Creep rate $=1245.957 \mathrm{nhs}+0.026 \mathrm{Po}+0.002$ Age $-0.016 \mathrm{In} . \mathrm{St}-0.302$} \\
\hline nhs & $\mathrm{r}^{2}=0.540$ & $\mathrm{p}<0.0001$ \\
\hline Po & $\mathrm{r}^{2}=0.583$ & $\mathrm{p}<0.0001$ \\
\hline Age & $\mathrm{r}^{2}=0.588$ & $\mathrm{p}=0.0536$ \\
\hline In.St & $r^{2}=0.590$ & $\mathrm{p}=0.0057$ \\
\hline Model & $\mathrm{r}^{2}=0.592$ & $\mathrm{p}<0.0001$ \\
\hline
\end{tabular}

The model predicts that creep rate will have a positive relationship with normalized hoop stress, porosity and age, and an inverse relationship with initial stiffness. Age was included in this model because of the contribution to explaining variability in the model. Initial stiffness explains an additional $0.7 \%$ of the variability after porosity is included in the model. The low $\mathrm{r}^{2}$ value for the whole model suggests that there is a lot of variability not explained by the control parameters. 


\subsection{Permanent Strain}

Permanent (plastic) strain was measured as the amount of strain remaining at the end of the two minutes of zero pressure (Utenkin and Sveschnikova, 1973; Fondrk et al., 1988; Nicolella et al., 1998). The two minutes during which the specimen was at zeroload was sufficient for the permanent strain to reach an equilibrium strain value as indicated by the asymptotic nature of the curve (Figure 5.22).

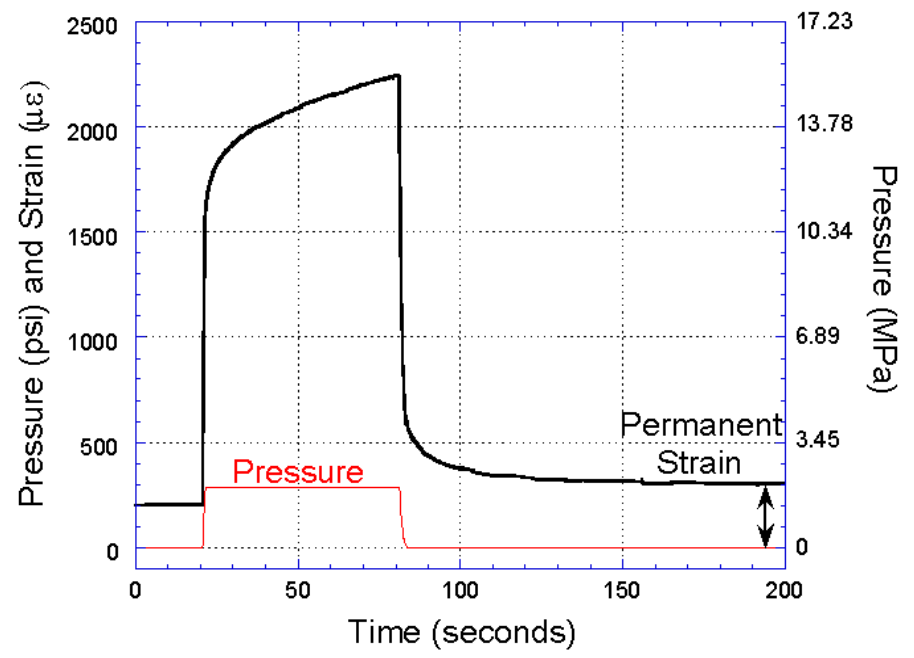

(A)

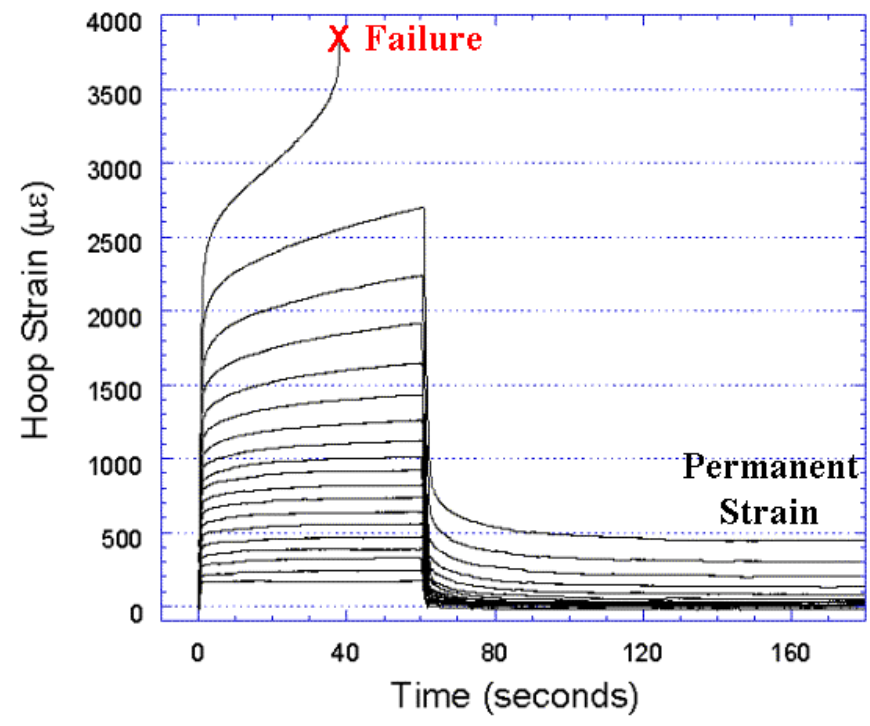

(B)

Figure 5.22 (A) Permanent strain, $300.04 \mu \varepsilon$, measured as the strain after 2 minutes of zero pressure (Test 8i). (B) Permanent strain increases with increasing levels of hoop stress. 
The stress levels for the permanent strain analysis were normalized as described above. The fit model platform in JMP was used to apply the standard least squares for general linear models. There were significant differences among the curve fits for the different quadrants, however, if the same equation was used to fit the responses for all quadrants, the loss in predictive ability was less than $1 \%\left(\mathrm{R}^{2}=0.722\right.$ versus $\left.\mathrm{R}^{2}=0.730\right)$. Based on this statistical analysis, the permanent strain from all four quadrants was analyzed as one group (Figure 5.23).

A fourth order polynomial was sufficient in providing the best fit of the data (Table 5.10). Exponential and power fits were not useful because of the consistent negative strain values at low levels of pressure. The relationship between plastic strain and normalized hoop stress was found to be significant, $\mathrm{p}<0.0001$.

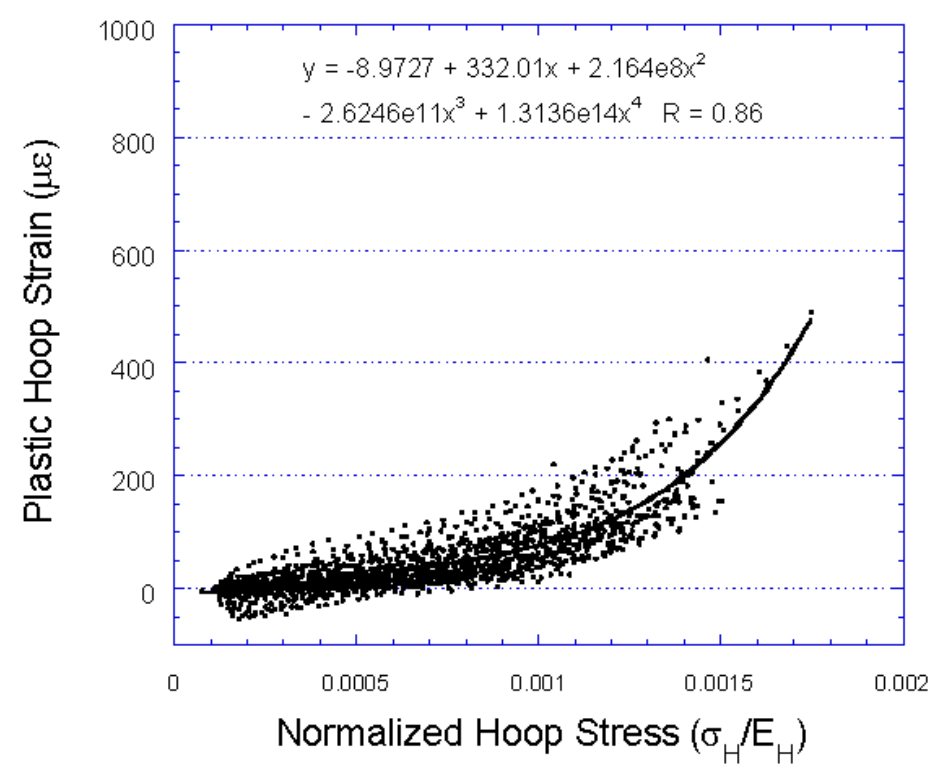

Figure 5.23 Permanent strain variation with hoop stress normalized with hoop stiffness from each stress level, $\mathrm{p}<0.0001$.

Table 5.10 Equations of various fits for permanent strain versus normalized hoop stress.

Fit

\begin{tabular}{|c|l|c|}
\hline Exponential & \multicolumn{1}{|c|}{-} & - \\
\hline Polynomial & $\mathrm{y}=-8.973+332.01 \mathrm{x}+2.16 \mathrm{E} 8 \mathrm{x}^{2}-2.625 \mathrm{E} 11 \mathrm{x}^{3}+1.314 \mathrm{E} 14 \mathrm{x}^{4}$ & 0.86 \\
\hline Power & \multicolumn{1}{|c|}{-} & - \\
\hline Linear & $\mathrm{y}=-47.119+1.406 \mathrm{E} 5 \mathrm{x}$ & 0.79 \\
\hline Logarithmic & $\mathrm{y}=579.28+163.6 \log (\mathrm{x})$ & 0.69 \\
\hline
\end{tabular}




\subsubsection{Permanent Strain Threshold}

An offset line was determined as described previously. The specimens exhibited linear permanent strain behavior during low stress levels (Figure 5.23). Permanent strain data below a normalized stress level of $0.0005\left(\sigma_{\mathrm{H}} / \mathrm{E}_{\mathrm{H}}<0.0005\right)$ were chosen for linear regression to model the initial behavior (Figure 5.24). The slope of the fit representing the linear behavior, (slope $=77107$ ), was used to create a line, offset to the upper bound of the $95 \%$ confidence interval.

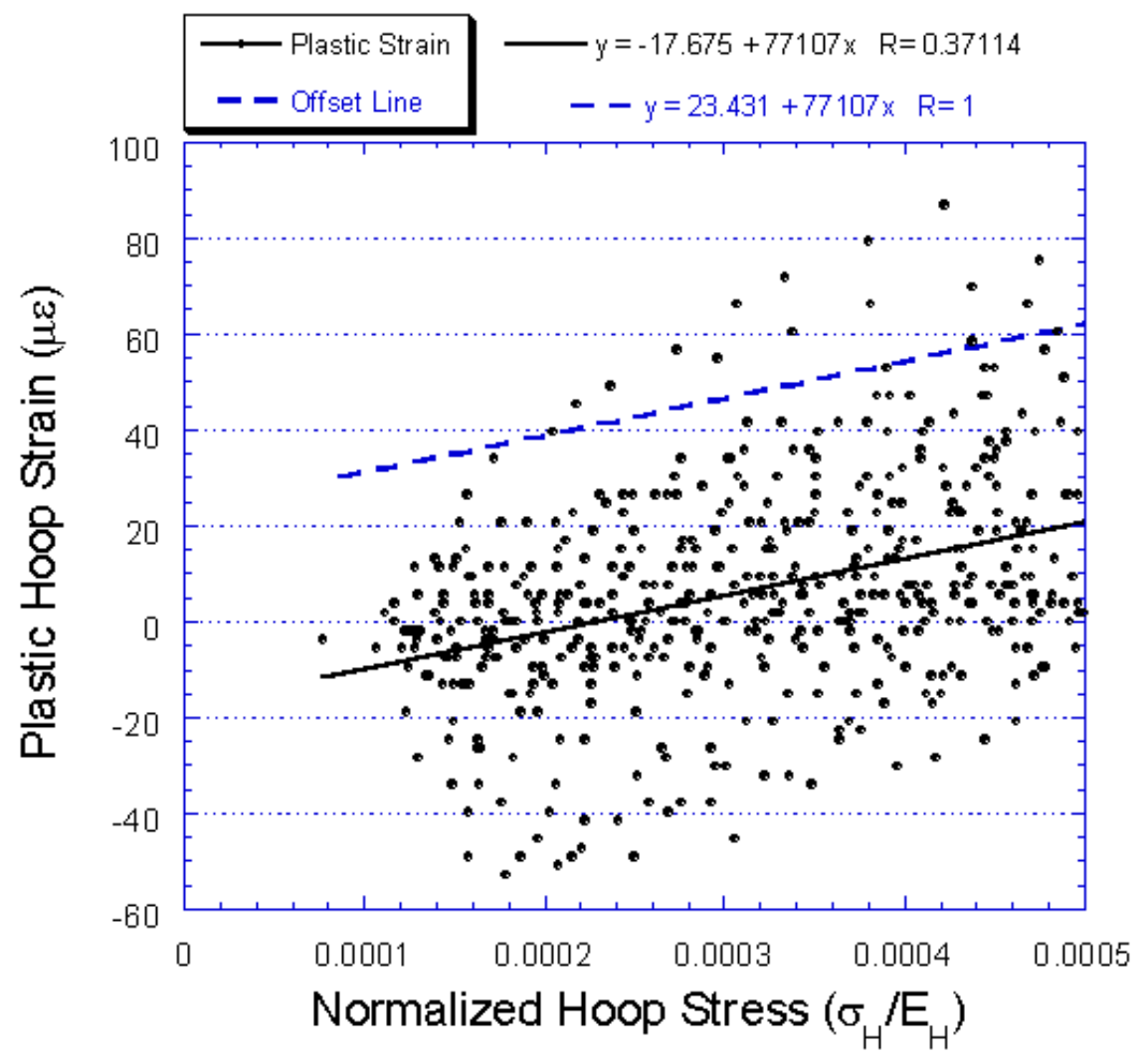

Figure 5.24 Plastic strain measured during the normalized hoop stress levels that resulted in linear behavior $\left(\sigma_{H} / E_{H}<0.0005\right)$. The dashed line was offset from the linear fit of the data represented by the solid line.

The intersection of the linear offset and exponential fit provides a threshold value of normalized hoop stress, 0.001170 , and permanent strain, $113.7 \mu \varepsilon$, at which the permanent strain begins to increase nonlinearly (Figure 5.25). 
(A)
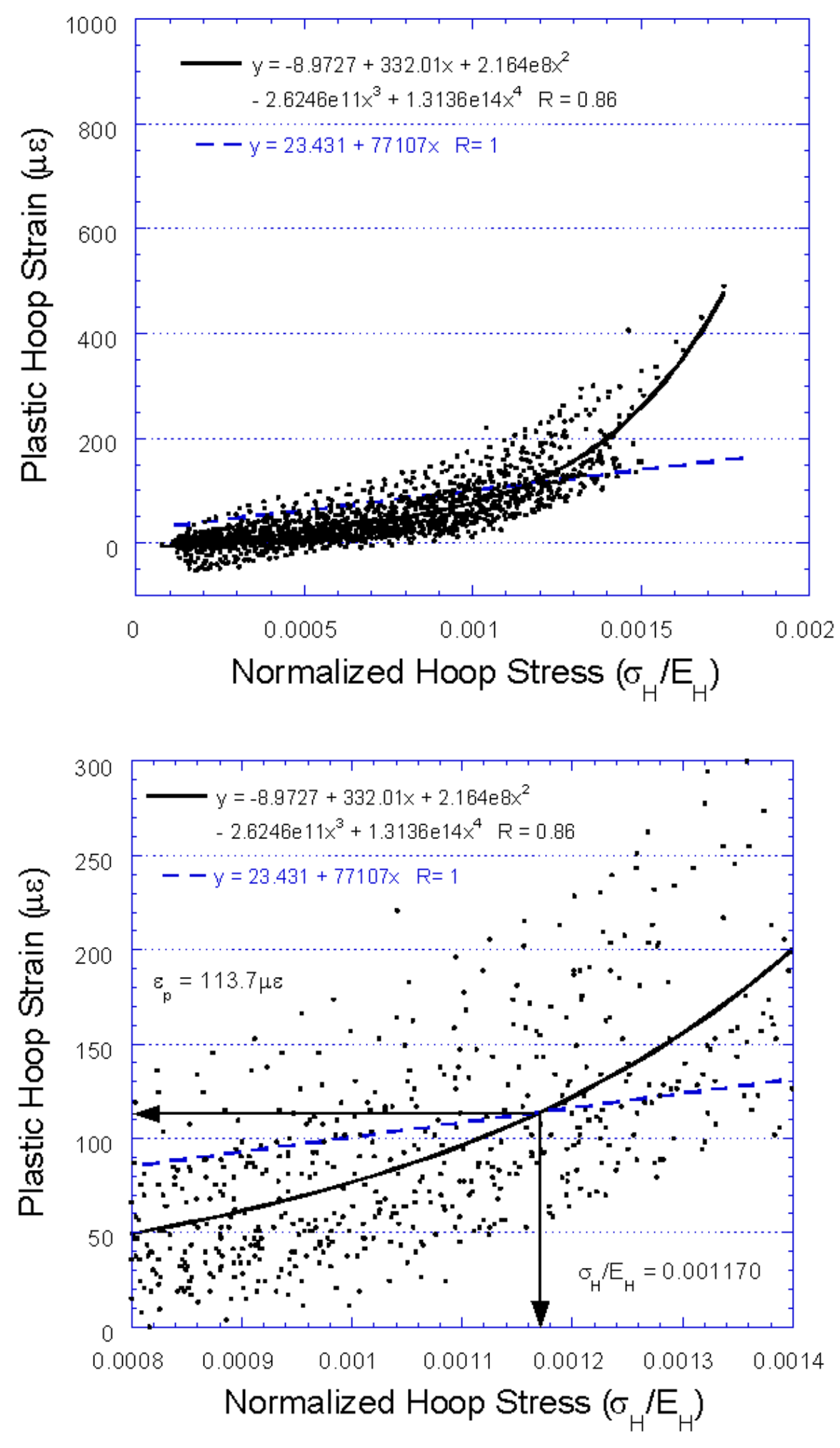

Figure 5.25 The plastic strain threshold was the intersection between the fourth order polynomial fit and the offset line. 


\subsubsection{Multivariable Model of Permanent Strain}

Stepwise regression analysis was used for scanning variables for including in a regression model. A multivariable model using the least squares procedure was used for estimating permanent strain $(\mu \varepsilon)$ (Table 5.11). The control parameters examined were normalized hoop stress, location, gender, age, porosity, osteon area, osteon density, single osteon area and initial stiffness. Those that were found suitable for a model were normalized hoop stress (nhs), location, age, osteonal area (On.Ar), single osteon area (s.On.Ar) (E-2) and osteon density (On.Dn).

Table 5.11 Multivariable model of permanent strain.

\begin{tabular}{|crr|}
\hline Permanent strain $=140210.7$ nhs -18.781 & s.On.Ar +12.339 Location $(\mathrm{P}-\mathrm{L})-11.498$ \\
On.Dn +0.214 Age +1.784 On.Ar +74.674 & \\
\hline nhs & $\mathrm{r}^{2}=0.629$ & $\mathrm{p}<0.0001$ \\
s.On.Ar & $\mathrm{r}^{2}=0.634$ & $\mathrm{p}=0.0238$ \\
Location (P-L) & $\mathrm{r}^{2}=0.643$ & $\mathrm{p}<0.0001$ \\
On.Dn & $\mathrm{r}^{2}=0.645$ & $\mathrm{p}=0.0003$ \\
Age & $\mathrm{r}^{2}=0.646$ & $\mathrm{p}=0.0092$ \\
On.Ar & $\mathrm{r}^{2}=0.648$ & $\mathrm{p}=0.0066$ \\
\hline Model & $\mathrm{r}^{2}=0.649$ & $\mathrm{p}<0.0001$ \\
\hline
\end{tabular}

The model predicts that permanent strain will develop with normalized hoop stress, age, osteon area and with decreasing osteon density and single osteon size. Osteon density, age and osteon area together only account for $0.5 \%$ of the variability in this model. 


\subsection{Material Assumption for Bone}

The objective of this research was to understand the time-dependent deformation in the hoop direction for cortical bone. The theory used to calculate stress in the bone cylinder was based on the bone being an elastic material. An alternate assumption based on these results was that the bone exhibits creep deformation governed by the NortonOdqvist flow rule:

$$
\begin{gathered}
\dot{\varepsilon_{\theta}}=\frac{k}{2} 3^{(1+n) / 2}\left(\frac{p_{i}}{n}\right)^{n}\left[\left(\frac{r_{o}}{r_{i}}\right)^{2 / n}-1\right]^{-n}\left(\frac{r_{o}}{r}\right)^{2} \\
\sigma_{\theta}=\frac{p_{i}}{\left(\frac{r_{o}}{r_{i}}\right)^{2 / n}-1}\left[1-\left(1-\frac{2}{n}\right)\left(\frac{r_{o}}{r}\right)^{2 / n}\right\rceil
\end{gathered}
$$

Hoop strain rate was experimentally determined at various levels of internal pressure. These values were entered into equation 2.7 and the parameters $\mathrm{k}, 0.00005$, and $\mathrm{n}, 1.39$, were determined using curve fitting techniques in JMP. Substituting these values into equations 2.7 and 2.8 provides a hoop strain rate and hoop stress relationship as a function of internal pressure and radius:

$$
\begin{aligned}
& \dot{\varepsilon}_{\theta}(\mu \varepsilon / s)=0.00385\left(\frac{p_{i}^{1.39}}{r^{2}}\right) \\
& \sigma_{\theta}=\frac{p_{i}}{0.3164}\left(1+\frac{14.738}{r^{1.439}}\right)
\end{aligned}
$$

The hoop stress equation reduces to the following at the inner and outer surface of the cylinder:

$$
\begin{aligned}
\sigma_{\theta, \text { inner }} & =4.986 \mathrm{p}_{\mathrm{i}} \\
\sigma_{\theta, \text { outer }} & =4.547 \mathrm{p}_{\mathrm{i}}
\end{aligned}
$$

Hoop stress determined by equation 2.5 is based on the assumption that the cylinder material is elastic:

$$
\sigma_{\theta}=\frac{r_{i}^{2} p_{i}}{r_{o}^{2}-r_{i}^{2}}\left(1+\frac{r_{o}^{2}}{r^{2}}\right)
$$

This equation reduced to the following at the inner and outer surface of a cylinder of the same geometry: 


$$
\begin{aligned}
& \sigma_{\theta, \text { inner }}=5.298 \mathrm{p}_{\mathrm{i}} \\
& \sigma_{\theta, \text { outer }}=4.298 \mathrm{p}_{\mathrm{i}}
\end{aligned}
$$

The percent difference between the two material assumptions was $6 \%$ or less at either surface (Figure 5.26).

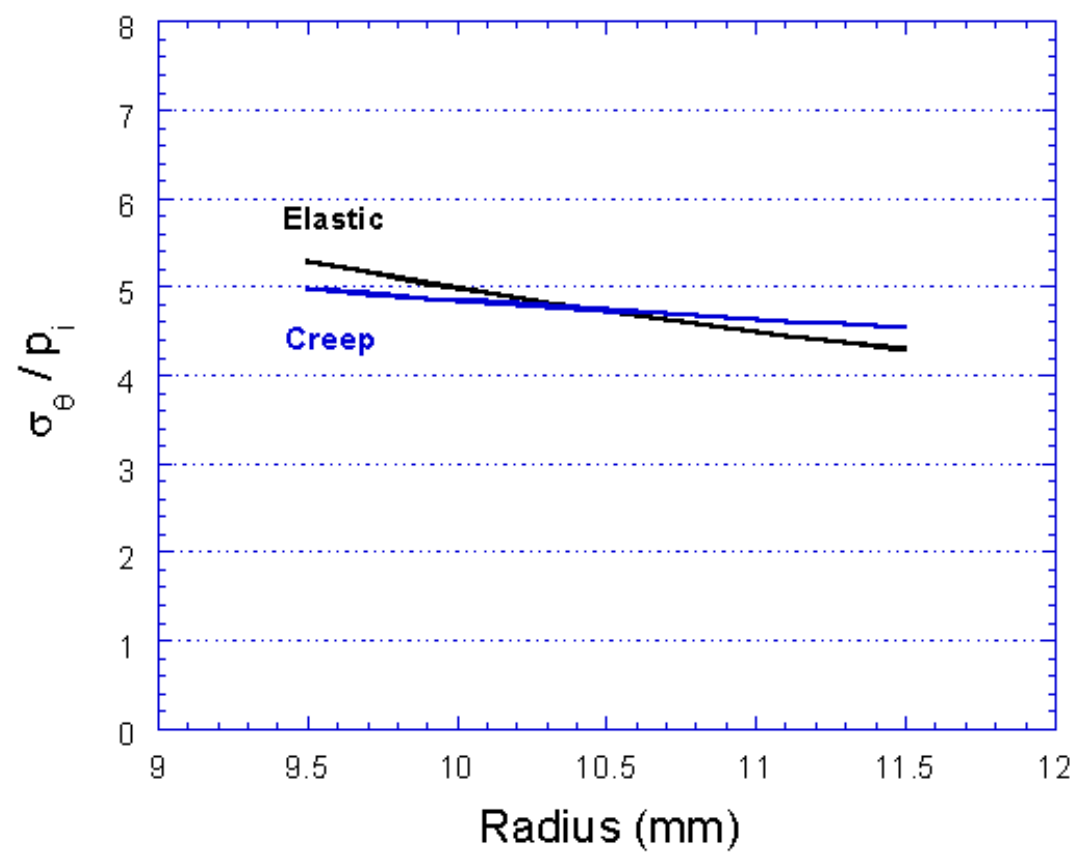

Figure 5.26 Hoop stress distribution based on elastic and creep deformation of a thick walled cylinder.

\subsection{Discussion}

Due to the limitations of the testing equipment, not all specimens were capable of being tested to failure. The solenoid valve worked reliably up to approximately $3.06 \mathrm{MPa}$ (444 psi). Seven specimens fractured below this pressure, while 8 specimens did not. Both groups of specimens showed a broad range in hoop strain throughout the working pressure range and the specimens were grouped together. Another limitation was the number of specimens that were available for testing. Due to the geometry of the specimens and the nature of cortical bone from the femoral mid-diaphysis, a limited number of specimens were machined. The specimen geometry was an idealized representation of the intact femur. This study examined material properties of the bone rather than structural properties, therefore, comparing these results directly to intact bone 
should be done with caution. A uniform cylinder with a thinner cross-section was used in this study compared to the cross section of the intact femur.

Normalizing the hoop stress was done to account for differences in cortical bone among the donors. Although this procedure may account for some donor differences, it is unlikely to be capable of accounting for all. The specimens were subjected to an idealized loading environment of a press-fit stem, only an internal radial load was applied to the specimens via internal pressure. A more complicated loading environment exists in vivo.

The cylindrical shape of the bone specimens and the test fixture provide a unique method to examine material properties of cortical bone. Previous attempts to determine hoop stiffness values using mechanical testing methods were done with rectangular specimens machined and loaded in the transverse plane. The mean initial hoop stiffness, 10.52 $\mathrm{GPa}$, the hoop stiffness from the initial pressure of $0.345 \mathrm{MPa}$ (50 psi) was within the range of previously reported values, 8.51 to $13.4 \mathrm{GPa}$, typically referenced in literature (Table 5.2). There were significant differences between quadrants with medial hoop stiffness being greater than the posterior and lateral quadrants. A difference among quadrants was expected since differences exist among the quadrants for longitudinal stiffness. Ashman et al. (1984) reported a lower stiffness in the posterior quadrant than the other three using an ultrasound technique. Rho et al. (1995) also found the posterior stiffness to be less than the anterior and medial quadrants using a similar technique.

Hoop stiffness was also determined over the entire range of applied pressure for each quadrant and was found to decrease due to damage during repeated load cycles. This loss in stiffness was similar to the loss of stiffness during fatigue loading reported in bone (Carter and Hayes, 1977; 1977a). It was suggested that small microcracks generated at the cement lines and at interlamellar bands were responsible. The cycles of load during the multi-phase testing protocol in this study may be analogous to a fatigue test at a very slow frequency.

The mean instantaneous stiffness, $10.52 \mathrm{GPa}$ and the mean static stiffness, 7.76 $\mathrm{GPa}$, were less than the values reported by Fondrk et al. (1988) of $13.1 \mathrm{GPa}$ and $15.2 \mathrm{GPa}$ for the static and instantaneous stiffness, respectively. This relationship was expected since values of hoop stiffness were less than longitudinal stiffness values reported in literature (Cowin et al., 1987) (Table 5.3) due to the anisotropy of cortical bone. The ratio 
of the static and instantaneous stiffness, 0.74, was smaller than the value determined by Fondrk et al. (1988) of 0.86 for longitudinal human bone. Fondrk et al. (1988) suggest that the ratio was an indication of the deviation from linear elastic behavior caused by viscoelastic effects and this deviation was not more than $15 \%$ of the total strain in the pre-threshold region. The lower ratio of hoop stiffness values determined in this study suggests that the linear elastic behavior of bone is more likely to be affected by viscoelastic effects in the hoop direction.

The creep strain, creep rate, and permanent strain all initially exhibited linear behavior at low stresses, until a particular stress level, or threshold, where they began to exhibit nonlinear exponential behavior at higher stresses. The values of normalized hoop stress, $0.00109,0.00115$ and 0.00117 for creep strain, creep rate and permanent strain, respectively, were all similar providing support for a threshold value in this range. Although the test specimens were an idealized geometry of the intact bone, the results suggest that there may be a similar threshold between linear and exponential creep strain, creep rate and permanent strain behavior in intact bone.

The normalized hoop stress thresholds $\left(\sigma_{\mathrm{H}} / \mathrm{E}_{\mathrm{H}}\right)$ may be converted back to hoop stress and internal pressure values with a few assumptions and calculations. First, the mean hoop stiffness $\left(\mathrm{E}_{\mathrm{H}}\right)$ from the entire pressure range, $9.79 \mathrm{GPa}(1.42 \mathrm{E}+06 \mathrm{psi})$ (Table 5.4) was used to obtain the hoop stress threshold for creep strain on the outer cylinder surface, 10.6 MPa (1545 psi). Secondly, using the mean specimen thickness of $2.01 \mathrm{~mm}$, the hoop stress value was converted to internal pressure, 2.49 $\mathrm{MPa}$ (362 psi), using equation 2.5. The normalized hoop stress thresholds for creep rate and permanent strain reduce to hoop stresses of $11.3 \mathrm{MPa}(1633 \mathrm{psi})$ and $11.4 \mathrm{MPa}$ (1661 psi), respectively, and to internal pressure values of $2.63 \mathrm{MPa}(382 \mathrm{psi})$ and $2.68 \mathrm{MPa}$ (389 psi), respectively. These values were approximations because the mean hoop stiffness value and the mean specimen thickness were used. These internal pressure values provided target pressure levels for the subsequent long-term creep tests (Chapter 6). The hoop creep rate stress threshold determined in this study, 11.3 MPa, was lower than the value determined by Fondrk et al. (1988) for human longitudinal bone specimens, $73 \mathrm{MPa}$.

Creep strain occurred at very low levels of hoop stress, as low as $1.38 \mathrm{MPa}$ (200 psi) (Figure 5.13). This was much less than the longitudinal stress level, approximately 
$50 \mathrm{MPa}$, needed to cause creep strain reported by Fondrk et al. (1988). This deformation can occur at low hoop stress levels and may change the press-fit between the implant and bone and result in a loss of initial implant fixation.

Jasty et al. (1993) measured hoop strains during simulated uncemented femoral arthroplasty. Insertion of a femoral component judged to be a "precise-fit" (size of prepared canal is the size of the femoral component) produced hoop strains ranging from 100 to $1100 \mu \varepsilon$. Insertion of a femoral component that was press-fitted into the prepared canal, $0.5 \mathrm{~mm}$ smaller than the implant, produced hoop strains ranging from 100 to 2400 $\mu \varepsilon$ and a mean of $1160 \mu \varepsilon$. The range of hoop strains measured during the test in this study was up to approximately $3000 \mu \varepsilon$. Intact bone generally has a greater cortical thickness than the cylindrical specimen thickness of $2 \mathrm{~mm}$, so that higher hoop strains should exist on the surface of the thinner specimens. The maximum hoop strains measured in this study were similar, therefore, to the surface of intact femoral bone suggesting that our load environment was causing clinically relevant strains.

The creep rate threshold determined in this study, $1.04 \mu \varepsilon / \mathrm{s}$, was less than the value of $10 \mu \varepsilon / \mathrm{s}$ determined by Fondrk et al. (1988). Differences were most likely due to the anisotropy of bone. Our creep rate was similar to the creep rate of approximately $1 \mu \varepsilon / \mathrm{s}$ that was deduced from Pattin et al. (1995) for longitudinally oriented bovine bone specimens. Determining whether creep rates below, at or above the threshold will eventually lead to creep-failure was the subject of the long-term creep tests (Chapter 6).

Based on work by Fondrk et al. (1988), an exponential fit of the data was expected to have a very sharp increase at a particular stress. Creep rates for longitudinal bovine bone determined by Pattin et al. (1995) had a relatively sharp increase in creep rate as well. Thus, the stress level boundary between linear and nonlinear behavior for the hoop creep rate was expected to be obvious. However, the nature of this data provided an exponential curve, similar to the longitudinal data, but with gradual curvature.

Permanent strain, as seen in this study, was suggested to be the result of damage at the ultrastructural level, between the collagen fibers and the mineral crystals (Fondrk et al., 1988). Specimens consistently had negative permanent strain values at initial pressure levels. The reason is unknown, however, it is most likely the affect of the bone microstructure settling or pores closing after the initial load. The specimen was 
pressurized and deformed during the first loading phase, then during the zero-pressure phase the elastic nature of the specimen caused it to return to its original state however due to minor reorganizations within the tissue on the microstructural level or collapsing porosity, the specimen experienced negative plastic strain.

Using the experimental creep rate data for human cortical bone, an equation for the hoop stress of a creep deforming cortical bone cylinder was derived. The hoop stress predicted by either a creep deforming bone cylinder or an elastic cylinder was different by $6 \%$ or less. If the cylinder was assumed to be elastic, the stress determined on the outer surface was under-predicted. An exact value of hoop stress was not essential for this portion of the study since hoop stress was normalized.

Based on the predictive models of creep strain, creep rate and permanent strain, various morphology parameters correlated well. Porosity was included in the creep strain and creep rate model and explained a large portion of the variability in both suggesting its influence on creep behavior. Both models predict that creep strain and creep rate will increase with increasing porosity. Age appears to correlate with creep rate and permanent strain however it did not explain much of the variability, possibly due to the small age range of the bone specimens. Initial stiffness had an inverse relationship in both the creep strain and creep rate models which was expected and explained only a small portion of variability after porosity was included in the models.

This study provides knowledge of hoop creep strain, creep strain rate and permanent strain which exhibited a change in behavior between low and high levels of hoop stress. Creep strain can occur at low hoop stress levels, or low intramedullary loads, and may change the press-fit between the implant and bone. The effects of the threshold behavior of cortical bone on the stability of press-fit implants is not known. 


\section{CHAPTER 6 \\ LONG-TERM CREEP TESTS}

\subsection{Introduction}

Human cortical bone is known to exhibit viscoelastic, a time-dependent, behavior in the longitudinal direction of long bones under various loading environments (Sedlin, 1965; Crowninshield et al., 1974; Knets and Vilks, 1975, Melnis and Laizan, 1978, Melnis et al., 1979; Melnis and Knets, 1981; Melnis et al., 1981; Carter and Caler, 1983; Fondrk et al., 1988, 1990; Caler and Carter, 1989; Mauch et al., 1992; Rimnac et al., 1993; Tanabe and Kobayashi, 1994; Pattin et al., 1995; Bowman et al., 1999). Cortical bone may be considered to have orthogonal symmetry (Ashman et al., 1984), therefore, it is necessary to investigate time-dependent behavior in the transverse direction. The timedependent response of bone to transverse loads has received less attention. Previous work involving the transverse time-dependent response of bone has been limited to the study of bovine bone loaded in compression (Utenkin and Sveschnikova, 1973; Tanabe and Kobayashi, 1994), tension (Crowninshield and Pope, 1974) and torsion (Bonfield and Li, 1964). Although these studies did not examine long-term creep behavior, the results suggest that the time-dependent response of bone differs with the direction of the applied load. Further study is needed to understand the creep behavior of bone to loads applied in the transverse direction. The objective was to determine the creep response of bone specimens under constant load until failure to establish a creep model and a relationship between time to failure and stress.

\subsection{Materials and Methods}

Eight fresh human femora were obtained from 6 male and 2 female cadavers (mean age $40 \pm 16$ years) (Table 6.1). From these femora a total of 15 right circular cylinders $19 \mathrm{~mm}$ in length, with a $19 \mathrm{~mm}$ inner wall diameter and a mean wall thickness of $2.03 \pm 0.02 \mathrm{~mm}$ were wet machined. Four unidirectional strain gages were mounted on the outer surface of the specimen on the anterior, posterior, lateral and medial aspects; all gages were oriented to measure hoop strain. 
Table 6.1 Distribution of specimens with respect to gender and age.

\begin{tabular}{|c|c|c|c|c|}
\hline Number & Specimen \# & $\begin{array}{c}\text { Test } \\
\#\end{array}$ & Gender & $\begin{array}{c}\text { Age } \\
\text { (years) }\end{array}$ \\
\hline 1 & $976090 \# 3$ & 19 & Male & 33 \\
\hline 2 & $6369 \# 2$ & 20 & Female & 38 \\
\hline 3 & $6369 \# 6$ & 21 & Female & 38 \\
\hline 4 & $0179 \# 1$ & 22 & Male & 57 \\
\hline 5 & $976136 \# 4$ & 23 & Female & 24 \\
\hline 6 & $976152 \# 1$ & 24 & Male & 35 \\
\hline 7 & $976089 \# 1$ & 25 & Male & 48 \\
\hline 8 & $6372 \# 3$ & 26 & Male & 22 \\
\hline 9 & $976090 \# 2$ & 27 & Male & 33 \\
\hline 10 & $976136 \# 2$ & 28 & Female & 24 \\
\hline 11 & $6372 \# 5$ & 29 & Male & 22 \\
\hline 12 & $6372 \# 1$ & 30 & Male & 22 \\
\hline 13 & $6369 \# 4$ & 31 & Female & 38 \\
\hline 14 & $6369 \# 1$ & 32 & Female & 38 \\
\hline 15 & $96174 \# 4$ & 33 & Male & 66 \\
\hline
\end{tabular}

The equipment for the long-term tests was reduced from the previous set-up for the short-term tests (Figure 6.1). The solenoid, vent, 2-way valve and orifice were removed from the line. The pressure transducer was also moved upstream in front of the safety valve. This set-up reduced the number of potential pressure leaks.

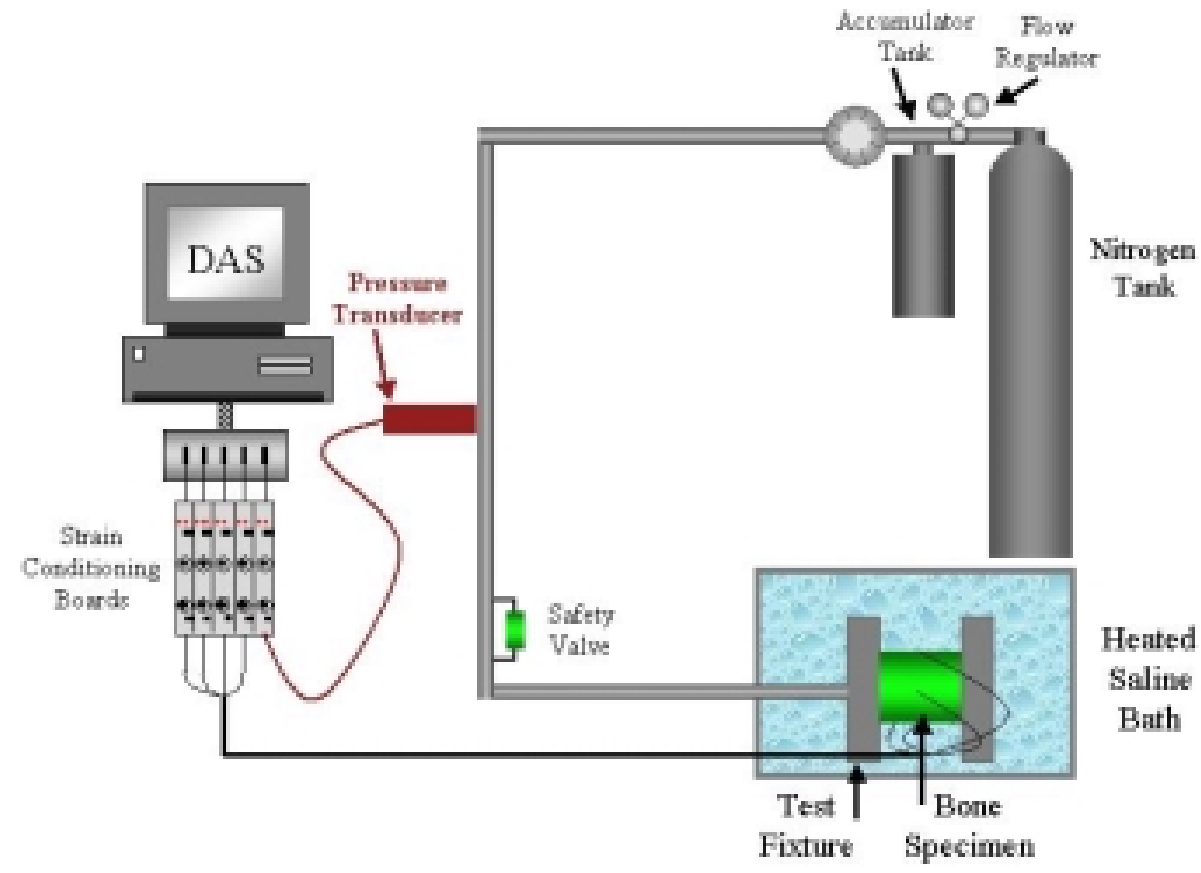

Figure 6.1 The experiment set-up for the long-term tests. 
A plug was placed on the end of the brake line before the specimen was attached. The desired pressure was pre-set using the pressure regulator. Once the accumulator was charged with the desired pressure level, the accumulator was sealed, the plug was removed from the line and the specimen was attached to the end of the line.

\subsubsection{Testing Protocol}

The DAS was initiated and the pressure was released from the accumulator. After a short period of time, the supply gas flow was rerouted so that it passed through the ball check safety valve. Once this step was complete, the nitrogen tank was sealed. The tank was sealed in case a rupture occurred anywhere in the system so that only the volume of gas in the accumulator would be released. The ball check safety valve prevented the accumulator from unnecessarily emptying during a rupture and also prevented a rapid release of the nitrogen gas.

Two data acquisition protocols were developed. The first program (cb2) captured data at a sampling rate of $10 \mathrm{~Hz}$ for 800 seconds to collect the nonlinear creep strain behavior early during the test. The quick sampling rate also captured the specimen pressurization allowing a hoop stiffness value to be determined from a hoop stress-strain curve. The second program (cb3) captured data at a sampling rate of $1 / 30 \mathrm{~Hz}$ for 24 hours then $1 / 60 \mathrm{~Hz}$ for 96 hours. The second program was restarted during a test when necessary. The specimens were loaded with constant pressure until failure except in one case when the test was terminated after 14 days (Test 23). Mauch et al. (1992) reported terminating creep tests of bovine bone and deer antler after approximately one day.

The strain gages experienced electrical shorting due to saline migration to the wires during tests that lasted several days. The DAS recorded a maximum negative value when a gage was shorted which made it obvious when a gage had been compromised. Loss of the gages occurred during the second stage of creep for the specimens. The time of failure was clear even from shorted gages because when the bone fractured the strains jumped to the maximum positive value indicating tensile fracture. Therefore, the time to failure was captured for each specimen as well as a significant portion of the steady state creep. 
Specimens with operational strain gages throughout the duration of the creep tests exhibited the three stages of creep commonly associated with engineering materials (Figure 6.2). The compressive strain during the third stage of creep for the anterior gage was observed in a previous study (Vanscoy, 1996) and believed to be a result of the opposite gage experience rapid tensile deformation.

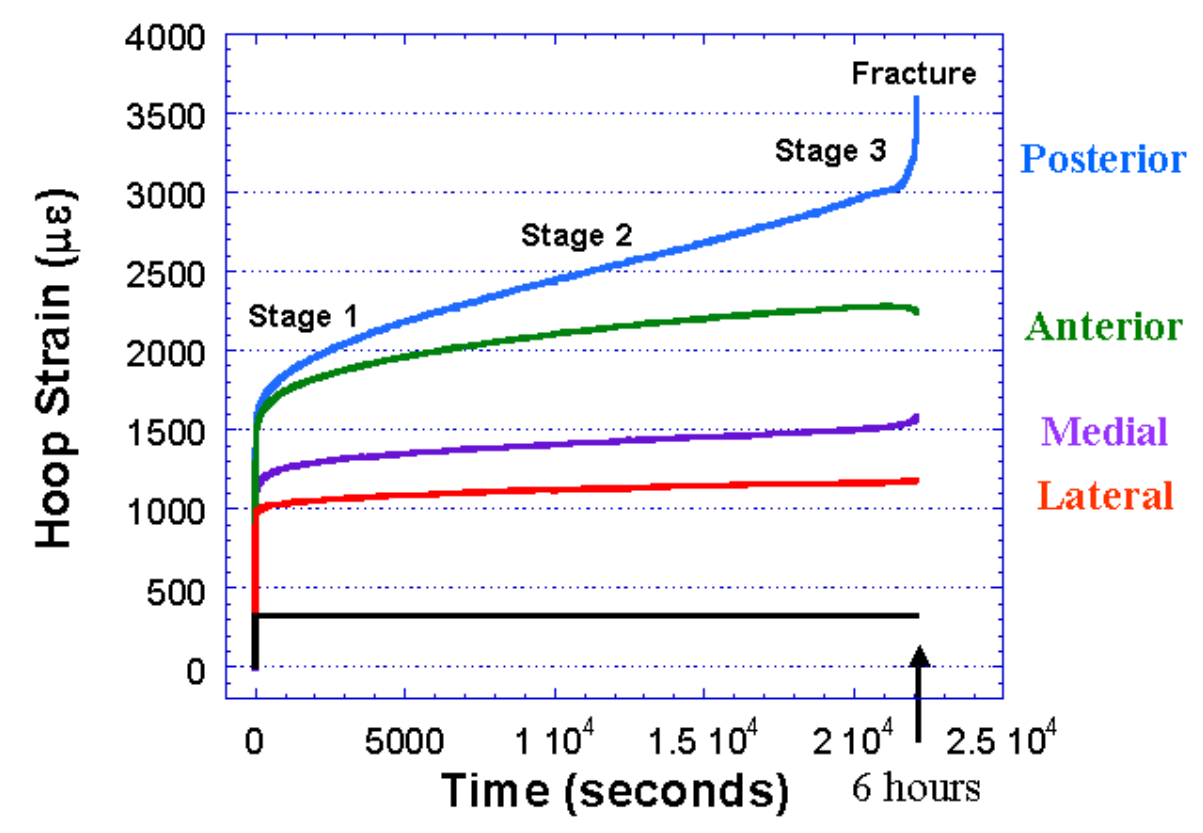

Figure 6.2 The three stages of creep strain for a specimen (Test 25) with internal pressure of $2.21 \mathrm{MPa}$ (321 psi). Creep failure occurred after 2.21E+04 seconds (6.14 hours).

\subsubsection{Morphological and Statistical Analysis}

The morphological analysis was conducted after testing as described in Chapter 5. Percent porosity, percent osteonal area, osteon density and average area of a single osteon were calculated using equations 5.1 - 5.4.

Statistical analysis was performed using JMP (SAS Institute, Cary, NC). Statistical significance was set as $\mathrm{p}<0.05$ or less. The following statistical procedures were used such as analysis of variance (ANOVA) for examining difference among bone quadrants, curve fitting with the nonlinear platform to determine the creep models, stepwise regression analysis for screening variables for inclusion in least squares procedures and regression to compare goodness of fit between observed and predicted strain from creep equations. 


\subsection{Time to Failure Results}

The values of internal pressure were chosen to span the pressure range of the threshold value of normalized hoop stress determined during the short-term tests. Seven pressure levels were chosen: 1.86 MPa (270 psi) (Tests 19, 21, 33), $2.07 \mathrm{MPa}$ (300 psi) (Test 23, 24), 2.20 MPa (320 psi) (Test 25, 26), $2.34 \mathrm{MPa}$ (340 psi) (Test 22, 30), 2.41 MPa (350 psi) (Test 20, 31), $2.48 \mathrm{MPa}$ (360 psi) (Test 29, 32) and $2.76 \mathrm{MPa}$ (400 psi) (Test 27, 28) (Table 6.2).

A specimen had four gages that recorded the same time to failure. Each gage, however, has a unique value of hoop stiffness and thus, a different normalized hoop stress $\left(\sigma_{\mathrm{H}} / \mathrm{E}_{\mathrm{H}}\right)$. In order to determine a relationship between normalized hoop stress and time to failure for a single specimen, only the normalized hoop stress value from the quadrant of the initial fracture was used. The quadrant responsible for the initial fracture was typically the quadrant that exhibited the most pronounced third stage of creep. In the cases where the third stage was not recorded by the gages due to the gage shorting or the third stage occurring in between data samples, the quadrant that exhibited the greatest deformation was chosen as the most likely quadrant of initial failure. The posterior, anterior and lateral quadrants were responsible for initial failure in 10,3 , and 2 specimens, respectively. 
Table 6.2 The constant internal pressure values for each specimen that were maintained until specimen failure, constant hoop stress values calculated using the internal pressure, and time to failure. Test \# 23 did not fail after 14 days.

\begin{tabular}{|c|c|c|c|c|c|}
\hline Number & $\begin{array}{c}\text { Specimen } \\
\#\end{array}$ & $\begin{array}{c}\text { Test } \\
\#\end{array}$ & $\begin{array}{c}\text { Constant } \\
\text { Internal } \\
\text { Pressure } \\
\text { MPa (psi) }\end{array}$ & $\begin{array}{c}\text { Constant } \\
\text { Hoop Stress } \\
\text { MPa (psi) }\end{array}$ & $\begin{array}{c}\text { Time to Failure } \\
\text { seconds } \\
\text { (days) }\end{array}$ \\
\hline 1 & $976090 \# 3$ & 19 & $1.83(264.82)$ & $7.64(1107.7)$ & $3.94 \mathrm{E}+05(4.56)$ \\
\hline 2 & $6369 \# 2$ & 20 & $2.41(350.18)$ & $10.21(1480.6)$ & $1.57 \mathrm{E}+02(0.0018)$ \\
\hline 3 & $6369 \# 6$ & 21 & $1.87(271.61)$ & $7.96(1154.3)$ & $6.35 \mathrm{E}+04(0.74)$ \\
\hline 4 & $0179 \# 1$ & 22 & $2.36(342.42)$ & $9.93(1439.9)$ & $7.96 \mathrm{E}+03(0.092)$ \\
\hline 5 & $976136 \# 4$ & 23 & $2.07(300.71)$ & $8.77(1271.4)$ & $1.20 \mathrm{E}+06(13.89)$ \\
\hline 6 & $976152 \# 1$ & 24 & $2.07(300.71)$ & $8.72(1264.5)$ & $9.54 \mathrm{E}+05(11.04)$ \\
\hline 7 & $976089 \# 1$ & 25 & $2.21(321.08)$ & $9.46(1372.4)$ & $2.21 \mathrm{E}+04(0.26)$ \\
\hline 8 & $6372 \# 3$ & 26 & $2.22(322.05)$ & $9.34(1354.2)$ & $8.98 \mathrm{E}+05(10.39)$ \\
\hline 9 & $976090 \# 2$ & 27 & $2.72(394.8)$ & $11.45(1660.1)$ & $1.78 \mathrm{E}+05(2.06)$ \\
\hline 10 & $976136 \# 2$ & 28 & $2.78(402.56)$ & $11.86(1720.6)$ & $5.33 \mathrm{E}+03(0.062)$ \\
\hline 11 & $6372 \# 5$ & 29 & $2.49(361.82)$ & $10.60(1537.7)$ & $3.56 \mathrm{E}+05(4.12)$ \\
\hline 12 & $6372 \# 1$ & 30 & $2.34(339.51)$ & $9.79(1420.2)$ & $9.78 \mathrm{E}+05(11.32)$ \\
\hline 13 & $6369 \# 4$ & 31 & $2.41(350.18)$ & $10.26(1488.3)$ & $1.81 \mathrm{E}+05(2.09)$ \\
\hline 14 & $6369 \# 1$ & 32 & $2.51(364.73)$ & $10.57(1533.7)$ & $6.39 \mathrm{E}+04(0.74)$ \\
\hline 15 & $96174 \# 4$ & 33 & $1.88(272.58)$ & $8.08(1171.4)$ & $4.67 \mathrm{E}+02(0.0054)$ \\
\hline
\end{tabular}

A significant relationship was not determined between internal pressure and time to failure (Figure 6.3). Several fits were attempted and the best was a line $(\mathrm{R}=0.25)$. Mauch et al. (1992) expressed difficulty finding an overall relationship between applied stress and time to failure for bovine bone and deer antler tissue. 


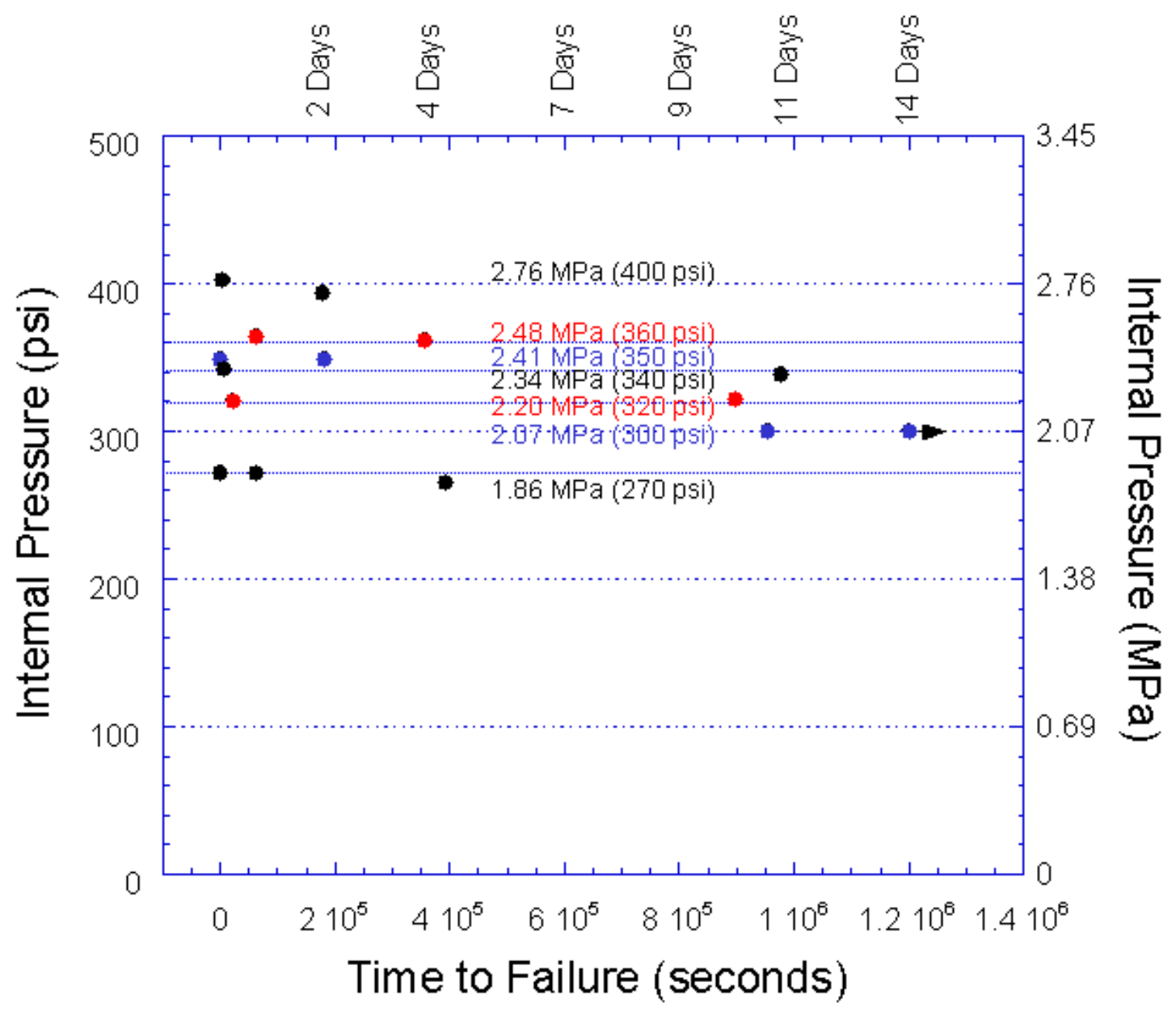

Figure 6.3 Pressure versus time to failure for all 15 specimens. There were seven general pressure levels: 1.86 MPa (270 psi), 2.07 MPa (300 psi), 2.20 MPa (320 psi), 2.34 MPa (340 psi), 2.41 MPa (350 psi), 2.48 MPa (360 psi) and 2.76 MPa (400 psi). Note one specimen (Test 23) did not fail after 14 days of constant pressure.

The hoop stress was normalized with hoop stiffness in an attempt to reduce the effects of donor variability (Caler and Carter, 1989; Mauch et al., 1992; Bowman et al., 1994, 1998, 1999) (Figure 6.4). Several fits were used to describe the nonsignificant relationship between normalized hoop stress and time to failure (Table 6.3). A logarithmic fit had the highest correlation coefficient and was nearly linear on a log-log plot (Figure 6.4B). 


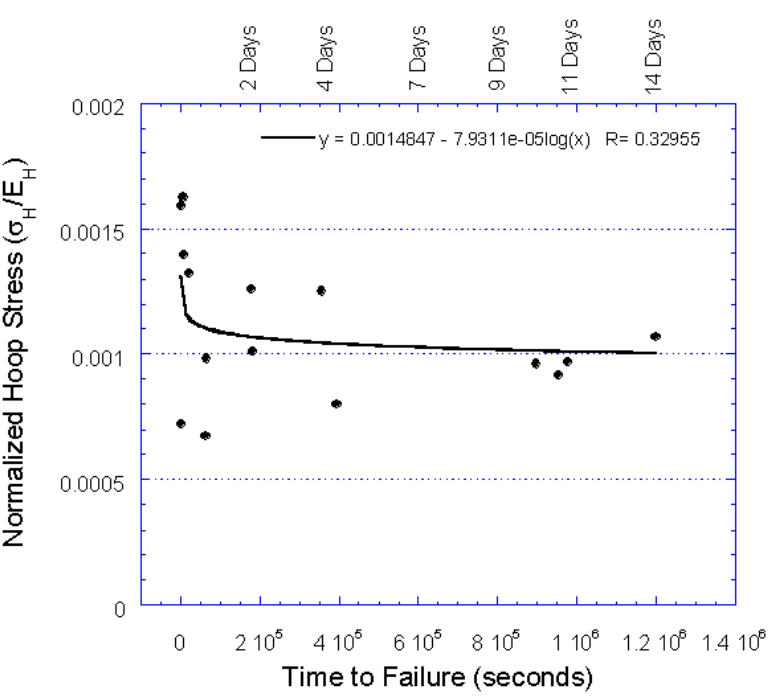

(A)

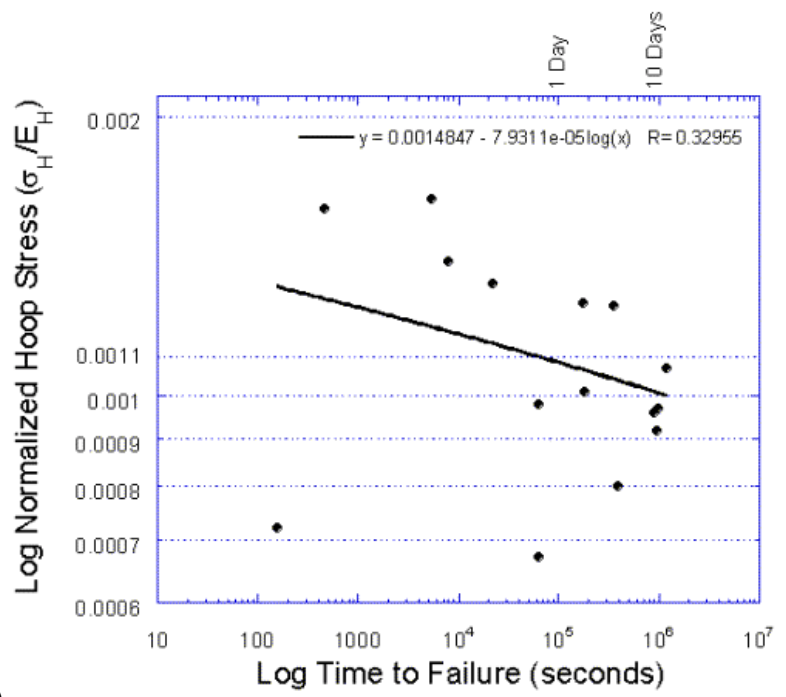

(B)

Figure 6.4 Normalized hoop stress and time to failure for all specimens. (A) A logarithmic fit was the best curve for the data. (B) A log-log plot of normalized hoop stress and time to failure allows the data at early times of failure to be observed. The fits of the data in Figure 6.4 were included, despite the non-significant relationship, for comparison to Figure 6.5.

Table 6.3 Equations of various fits for normalized hoop stress versus time to failure.

Fit Equation $\mathbf{R}$

\begin{tabular}{|l|l|l|}
\hline Logarithmic & $\mathrm{y}=0.00149-7.931 \mathrm{E}-05 \log (\mathrm{x})$ & 0.330 \\
\hline Power & $\mathrm{y}=0.00137 * \mathrm{x}^{\wedge}(-0.022758)$ & 0.319 \\
\hline Exponential & $\mathrm{y}=0.00113 * \mathrm{e}^{\wedge}(-1.504 \mathrm{E}-07 \mathrm{x})$ & 0.318 \\
\hline Linear & $\mathrm{y}=0.00118-2.14 \mathrm{E}-10 \mathrm{x}$ & 0.313 \\
\hline
\end{tabular}


Two data points had high stiffness values and thus low normalized hoop stress values (Figure 6.4B, Test \# 20 and 21). Both were removed and a significant relationship was determined between normalized hoop stress and time to failure $(p=0.026)$ (Figure 6.5). A logarithmic curve produced the best fit (Table 6.4).

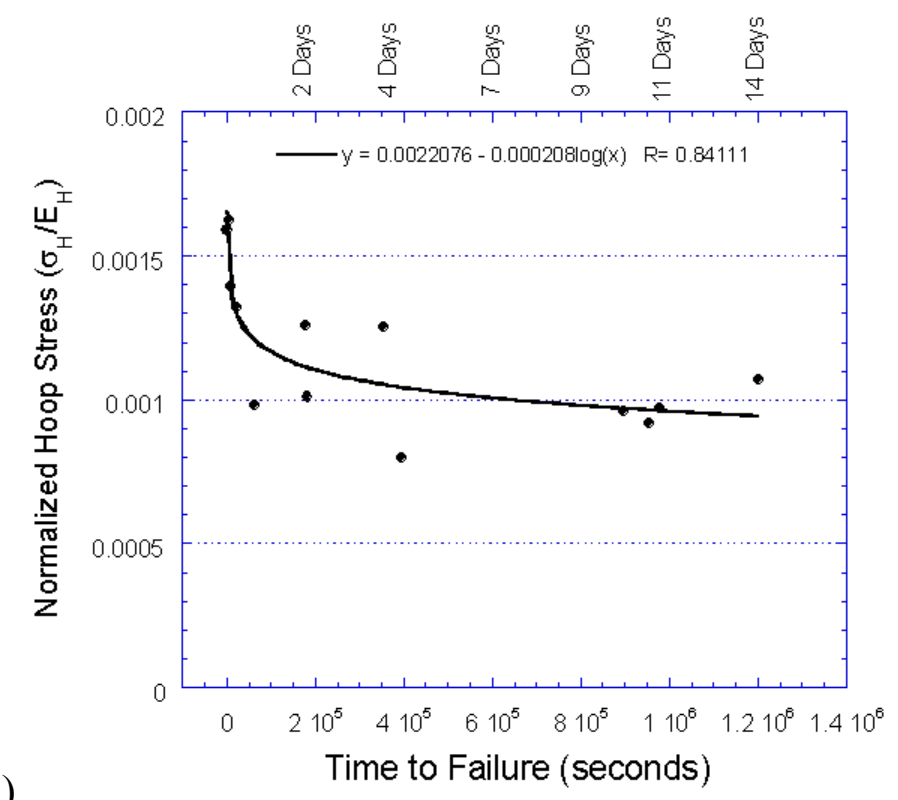

(A)

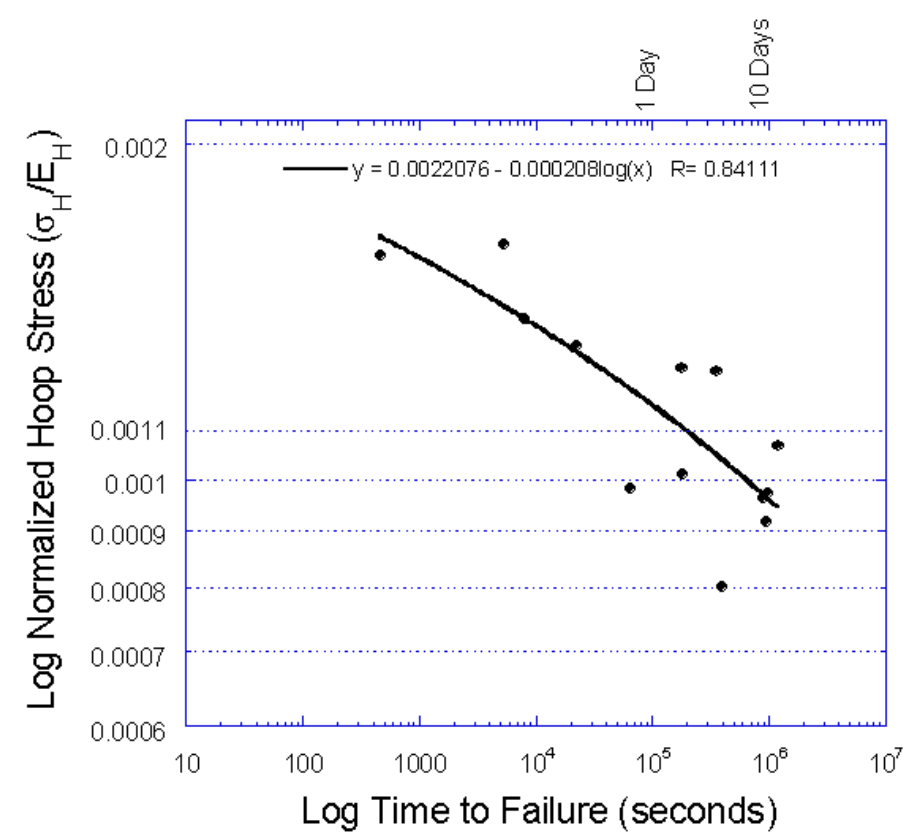

(B)

Figure 6.5 A significant relationship between normalized hoop stress and time to failure excluding Test \# 20 and 21, p=0.026, on a linear plot (A) and a logarithmic plot (B). 
Table 6.4 Equations of various fits for normalized hoop stress versus time to failure excluding Test \# 20 and 21.

\begin{tabular}{|l|l|c|}
\multicolumn{1}{c}{ Fit } & \multicolumn{1}{c}{ Equation } & R \\
\hline Logarithmic & $\mathrm{y}=0.00221-0.000208 \log (\mathrm{x})$ & 0.841 \\
\hline Power & $\mathrm{y}=0.00266^{*} \mathrm{x}^{\wedge}(-0.073339)$ & 0.839 \\
\hline Exponential & $\mathrm{y}=0.00129 * \mathrm{e}^{\wedge}(-2.992 \mathrm{E}-07 \mathrm{x})$ & 0.631 \\
\hline Linear & $\mathrm{y}=0.00131-3.6325 \mathrm{E}-10 \mathrm{x}$ & 0.613 \\
\hline
\end{tabular}

Caler and Carter (1989) reported time to failure for human femoral cortical bone specimens that were tested under constant tensile stress. Their data were linear on a loglog plot similar to power law creep behavior of materials. They used a power law equation to describe the relationship between normalized longitudinal stress (range $\left.0.002<\sigma_{\mathrm{L}} / \mathrm{E}_{\mathrm{L}}<0.006\right)$ versus time to failure:

$$
\mathrm{t}_{\mathrm{f}}=\mathrm{A}\left(\sigma_{\mathrm{L}} / \mathrm{E}_{\mathrm{L}}\right)^{-\mathrm{B}}
$$

where $t_{f}$ is time to failure, $\sigma_{L} / E_{L}$ is normalized longitudinal stress, $A$ is the creep coefficient (seconds) and B is the creep exponent. Including the constants from their experimental data the equation is:

$$
\mathrm{t}_{\mathrm{f}}=1.45 \mathrm{E}-36\left(\sigma_{\mathrm{L}} / \mathrm{E}_{\mathrm{L}}\right)^{-15.81} \quad\left(\mathrm{R}^{2}=0.95\right)
$$

From the power fit of the normalized hoop stress in this study $\left(0.00066<\sigma_{\mathrm{H}} / \mathrm{E}_{\mathrm{H}}<0.0016\right)$ (Table 6.4) is:

$$
\sigma_{\mathrm{H}} / \mathrm{E}_{\mathrm{H}}=0.0026549 \mathrm{t}_{\mathrm{f}}^{-0.073339}
$$

Solving for $\mathrm{t}_{\mathrm{f}}$, the following equation is derived:

$$
\mathrm{t}_{\mathrm{f}}=7.52 \mathrm{E}-36\left(\sigma_{\mathrm{H}} / \mathrm{E}_{\mathrm{H}}\right)^{-13.64}
$$

The power relationship from Caler and Carter's (1989) longitudinal creep failure data for the normalized stress level in this study $\left(0.00066<\sigma_{\mathrm{H}} / \mathrm{E}_{\mathrm{H}}<0.0016\right)$ shows that for any level of normalized stress in this range, creep-failure will take longer to occur for bone in the longitudinal direction than in the hoop direction (Figure 6.6). 


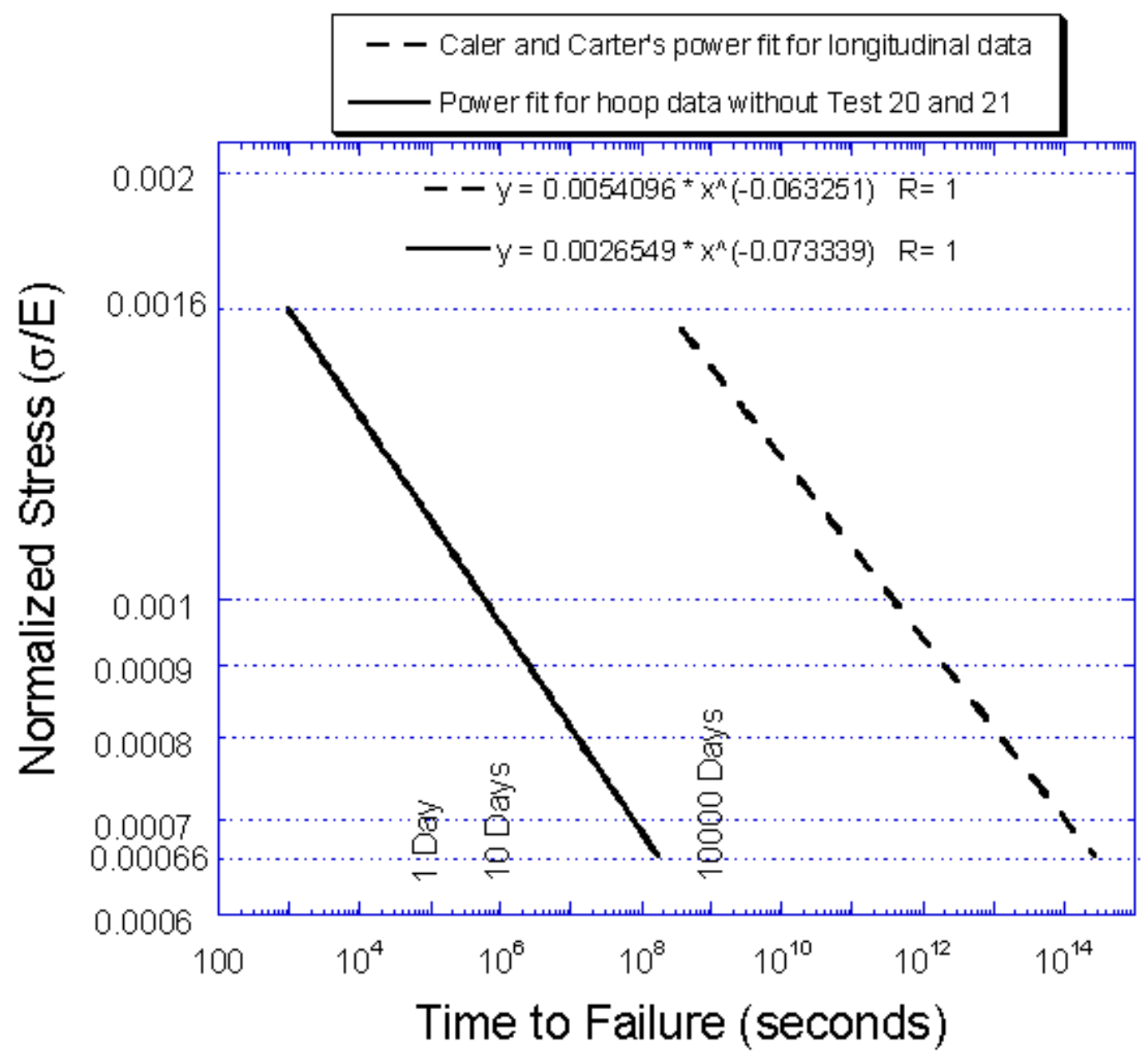

Figure 6.6 Caler and Carter's (1989) power fit for longitudinal creep fracture for the normalized stress level in this study compared to the power fit for hoop creep fracture for hoop specimens.

\section{Multivariable Model for Time to Failure}

Stepwise regression analysis was used for scanning variables for including in a regression model excluding data from Test \# 20 and 21. A multivariable model using the least squares procedure was used for estimating time to failure (seconds) (Table 6.5). The control parameters examined were normalized hoop stress, location, gender, age, porosity, osteon area, osteon density, single osteon area and initial stiffness. Those that 
were found suitable for a model were normalized hoop stress (nhs), location (L-P), gender, porosity (Po) and osteon density (On.Dn).

Table 6.5 Multivariable model of time to failure.

\begin{tabular}{|ccc|}
\hline $\begin{array}{c}\text { Time to failure }=-346828.9 \\
\text { On.Dn }-2.7226 \mathrm{E} 9 \text { nhs }+597039.77\end{array}$ & Location (L-P) - \\
449791 Gender -69734.72 Po +10468769 & $\mathrm{p}=0.0062$ \\
\hline On.Dn & $\mathrm{r}^{2}=0.418$ & $\mathrm{p}=0.0018$ \\
nhs & $\mathrm{r}^{2}=0.515$ & $\mathrm{p}=0.0429$ \\
Location (L-P) & $\mathrm{r}^{2}=0.685$ & $\mathrm{p}=0.0054$ \\
Gender & $\mathrm{r}^{2}=0.746$ & $\mathrm{p}=0.0336$ \\
Po & $\mathrm{r}^{2}=0.944$ & $\mathrm{p}=0.0033$ \\
\hline Model & $\mathrm{r}^{2}=0.960$ & \\
\hline
\end{tabular}

The model predicts that time to failure has an inverse relationship with osteon density, normalized hoop stress, gender and porosity. The parameters included in this model explained much of the model variability. Morphology parameters, osteon density and porosity, were important in the model for predicting time to failure. Porosity alone explained almost $20 \%$ of the variability and osteon density explained $42 \%$.

\subsection{Creep Model Results}

The creep specimens in this study are under a unique loading environment allowing the hoop deformation to be measured as a result of different hoop stress levels. With our results from these creep tests a creep model was derived to characterize hoop deformation as a function of hoop stress and time. The formulation of a creep model allows for long term performance prediction using short term tests, creep strains at various stress levels can be predicted, and creep can be incorporated into finite element models.

The strain data from each quadrant captured the creep behavior for each of the 15 tests (Figure 6.7). The creep function modeled the behavior including the nonlinear region during the first stage beginning at the point at which the internal pressure became constant to the beginning of the third stage when the strain rate began to increase. In 
some cases the strain gages short circuited and meaningful strain was no longer measured.

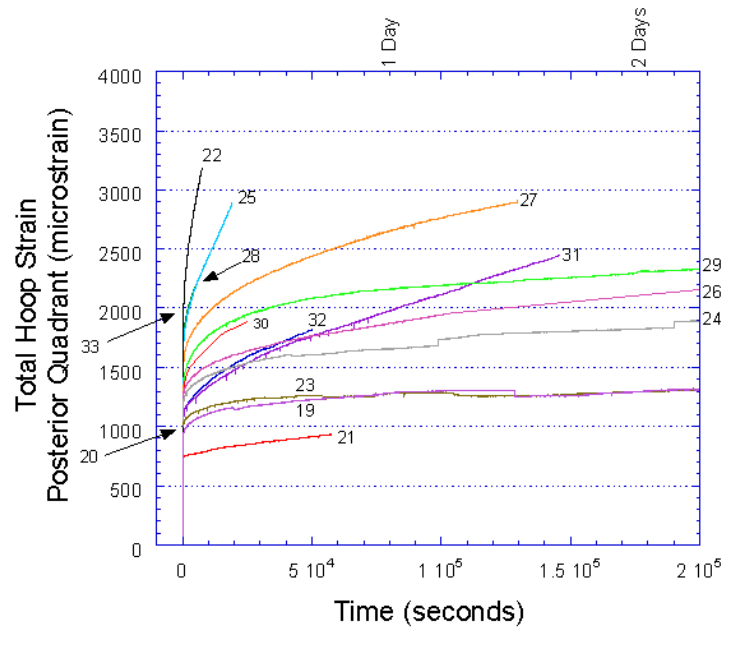

(A)

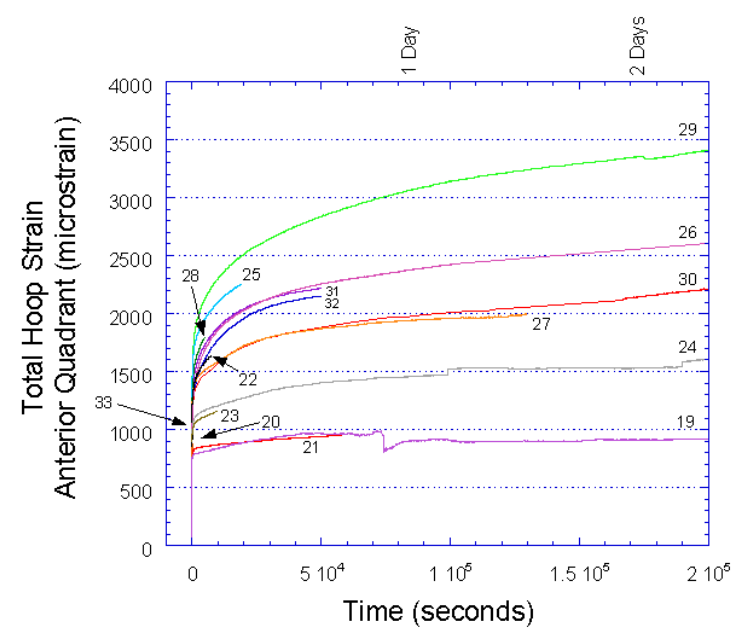

(C)

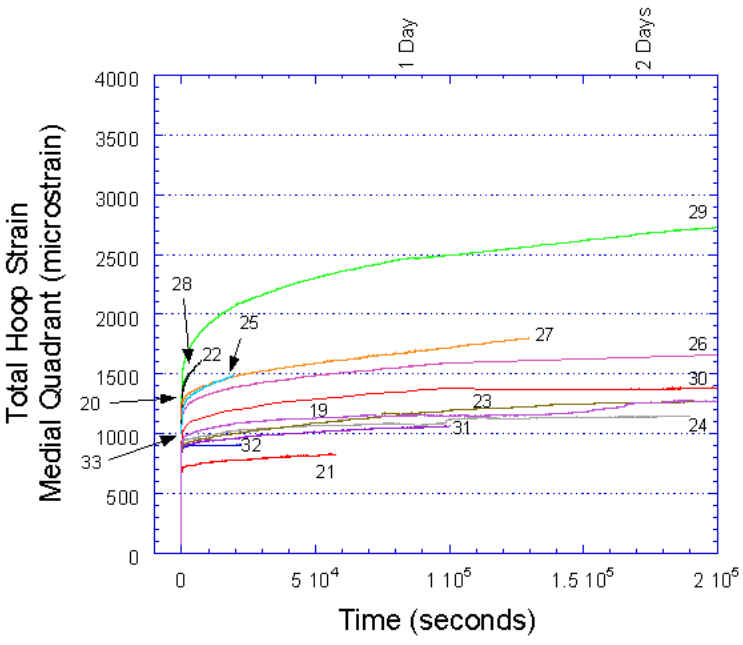

(B)

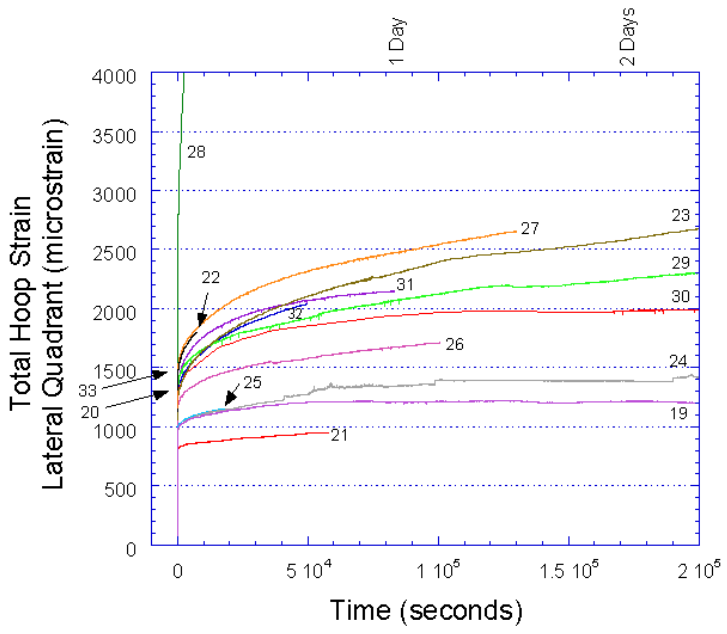

(D)

Figure 6.7 Hoop creep strain from each quadrant for all 15 specimens. The numbers in the plots represent the test number (19-33). The end of the strain-traces was either where the strain entered the third stage of creep or where the strain gage failed due to saline migration. The range of time on the $\mathrm{x}$-axis was chosen to allow the strain at early times to be observed. The specimen strain labeled with an arrow ends at the tip of the arrow. 


\subsubsection{The General Creep Model}

A FORTRAN program was used to select every $13^{\text {th }}$ data row to create a file of a reduced size of creep strain data. Reducing the size of the data for file management was not expected to effect the results considering the sampling rate used in both data collection protocols. The data sets were then aligned vertically into columns of time, constant stress, and strain in a single file in JMP.

The nonlinear curve fit option in JMP was used to fit a power function of the following form:

$$
\varepsilon=\mathrm{p} 1 \sigma^{\mathrm{p} 2} \mathrm{t}^{\mathrm{p} 3}
$$

where $\mathrm{p} 1, \mathrm{p} 2$ and $\mathrm{p} 3$ were the parameters to be solved, $\varepsilon$ is strain in the units of microstrain, $\sigma$ is hoop stress in the units of $\mathrm{MPa}$ and $\mathrm{t}$ is time in seconds. Initial values were input for the parameters $(\mathrm{p} 1=0.01155, \mathrm{p} 2=1.538, \mathrm{p} 3=0.0 .07787)$ and the model converged in an objective function of the following form:

$$
\varepsilon=24.40 \sigma^{1.54} \mathrm{t}^{0.078}
$$

for all specimens and all quadrants combined (if $\sigma$ is in the units of psi then $p 1=0.012$ ). Additional power functions were then determined for each quadrant:

$$
\begin{array}{ll}
\text { Posterior } & \varepsilon=50.31 \sigma^{1.33} \mathrm{t}^{0.055} \\
\text { Medial } & \varepsilon=41.33 \sigma^{1.27} \mathrm{t}^{0.069} \\
\text { Anterior } & \varepsilon=11.27 \sigma^{1.80} \mathrm{t}^{0.10} \\
\text { Lateral } & \varepsilon=17.94 \sigma^{1.71} \mathrm{t}^{0.075}
\end{array}
$$

(if $\sigma$ is in the units of psi then $\mathrm{p} 1=0.068,0.075,0.0015$ and 0.0037 for the posterior, medial, anterior and lateral quadrants, respectively)

The nonlinear curve fit option was also used to fit an exponential function of the following form:

$$
\varepsilon=\mathrm{p} 1 \mathrm{e}^{(\mathrm{p} 2 \sigma)} \mathrm{t}^{\mathrm{p} 3}
$$

where $\mathrm{p} 1, \mathrm{p} 2$ and $\mathrm{p} 3$ are the parameters to be solved, $\varepsilon$ is strain in the units of microstrain, hoop stress is in the units of $\mathrm{MPa}$ and time is seconds. Initial values were input for the parameters $(\mathrm{p} 1=200, \mathrm{p} 2=0.001, \mathrm{p} 3=0.01)$ and the model converged in an objective function of the following form:

$$
\varepsilon=174.57 \mathrm{e}^{(0.16 \sigma)} \mathrm{t}^{0.078}
$$


for all the specimens and all quadrants combined (if $\sigma$ is in the units of psi then $\mathrm{p} 2=0.0011)$. Additional exponential functions were then determined for each quadrant:

$$
\begin{array}{ll}
\text { Posterior } & \varepsilon=279.88 \mathrm{e}^{(0.13 \sigma)} \mathrm{t}^{0.055} \\
\text { Medial } & \varepsilon=208.11 \mathrm{e}^{(0.13 \sigma)} \mathrm{t}^{0.069} \\
\text { Anterior } & \varepsilon=116.71 \mathrm{e}^{(0.18 \sigma)} \mathrm{t}^{0.10} \\
\text { Lateral } & \varepsilon=148.88 \mathrm{e}^{(0.18 \sigma)} \mathrm{t}^{0.075}
\end{array}
$$

(if $\sigma$ is in the units of psi then $\mathrm{p} 2=0.00092,0.00089,0.0012$ and 0.0012 for the posterior, medial, anterior and lateral quadrants, respectively)

These creep models which include all quadrants of all 15 specimens represent the greatest variability of the bone material due to the inclusion of the specimens that exhibited high levels of creep strain.

\subsubsection{The Short-Term Creep Model}

The specimens were then separated into two groups to develop a creep model to characterize the behavior during the initial 6 hours of testing. The second creep model was developed to characterize the behavior over a period of several days. The time of 6 hours was chosen based on the time to failure distribution of the 15 specimens.

All of the specimens were included to develop the creep function to model the behavior during the initial 6 hours. There were 4 quadrants that exhibited especially high strain and were removed, Test 22 posterior (22P), Test 28 lateral, (22L), Test 33 posterior (33P) and Test 33 lateral (33L) (see Figure 6.7). A power law was used to model the behavior where $\varepsilon$ is strain in the units of microstrain, $\sigma$ is hoop stress in the units of $\mathrm{MPa}$ and $t$ is time in seconds. The objective function converged to the following:

$$
\varepsilon=25.24 \sigma^{1.48} \mathrm{t}^{0.085}
$$

(if $\sigma$ is in the units of psi then $\mathrm{p} 1=0.016$ ). Additional power functions were determined for each quadrant:

$$
\begin{array}{ll}
\text { Posterior } & \varepsilon=28.96 \sigma^{1.47} \mathrm{t}^{0.072} \\
\text { Medial } & \varepsilon=41.33 \sigma^{1.27} \mathrm{t}^{0.069} \\
\text { Anterior } & \varepsilon=11.27 \sigma^{1.80} \mathrm{t}^{0.10} \\
\text { Lateral } & \varepsilon=43.54 \sigma^{1.27} \mathrm{t}^{0.084}
\end{array}
$$


(if $\sigma$ is in the units of psi then $\mathrm{p} 1=0.019,0.075,0.0015$ and 0.080 for the posterior, medial, anterior and lateral quadrants, respectively). The medial and anterior creep models are the same as the general creep model above (equations $6.9,6.10$ ) because only posterior and lateral quadrants were removed.

The nonlinear curve fit option was also used to develop an exponential function:

$$
\varepsilon=168.00 \mathrm{e}^{(0.15 \sigma)} \mathrm{t}^{0.085}
$$

(if $\sigma$ is in the units of psi then $\mathrm{p} 2=0.0010$ ). Strain from $22 \mathrm{P}, 28 \mathrm{~L}, 33 \mathrm{P}$ and $33 \mathrm{~L}$ were not included. Additional exponential functions were determined for each quadrant:

$$
\begin{array}{ll}
\text { Posterior } & \varepsilon=185.75 \mathrm{e}^{(0.15 \sigma)} \mathrm{t}^{0.072} \\
\text { Medial } & \varepsilon=208.11 \mathrm{e}^{(0.13 \sigma)} \mathrm{t}^{0.069} \\
\text { Anterior } & \varepsilon=116.71 \mathrm{e}^{(0.18 \sigma)} \mathrm{t}^{0.10} \\
\text { Lateral } & \varepsilon=214.83 \mathrm{e}^{(0.13 \sigma)} \mathrm{t}^{0.084}
\end{array}
$$

(if $\sigma$ is in the units of psi then $\mathrm{p} 2=0.0010,0.00089,0.0012$ and 0.00091 for the posterior, medial, anterior and lateral quadrants, respectively).

The power and exponential creep models were plotted at each of the seven hoop stress levels (corresponding target pressure levels) (Figure 6.8). These hoop stress levels were used in the creep functions to model the behavior at each stress level. The creep models can then be compared to the measured creep strain at approximately equal stress levels. Each specimen had a unique thickness and was pressurized to a slightly different pressure level for each test, thus resulting in different hoop stress levels (Table 6.2).

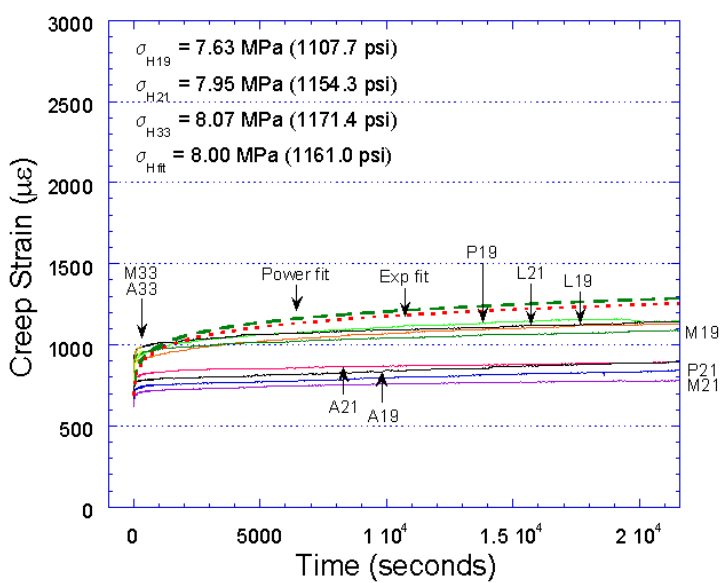

(A)

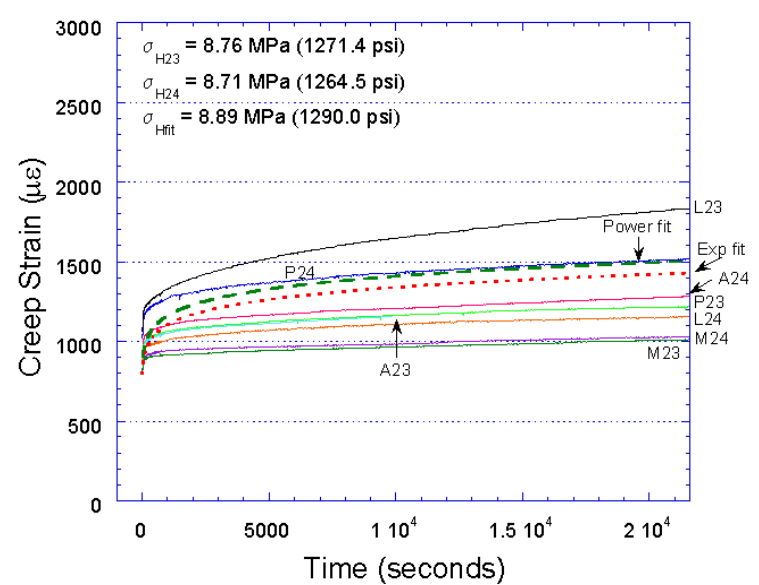

(B) 


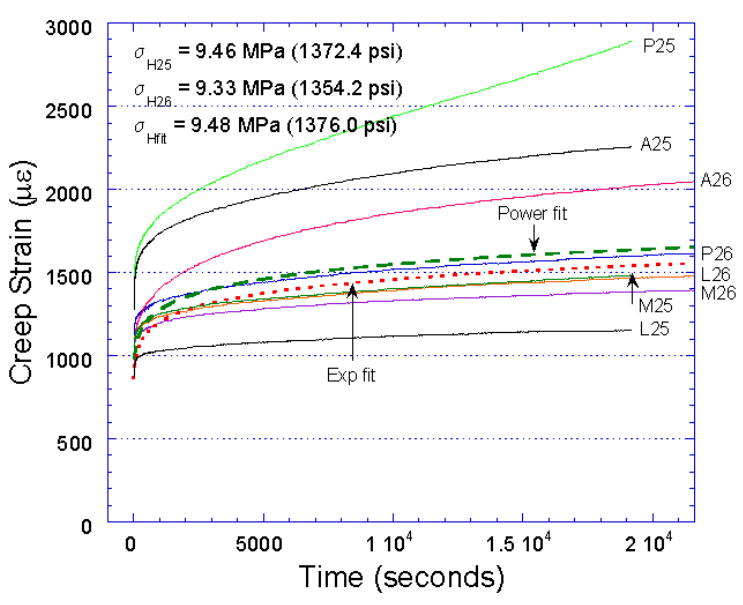

(C)

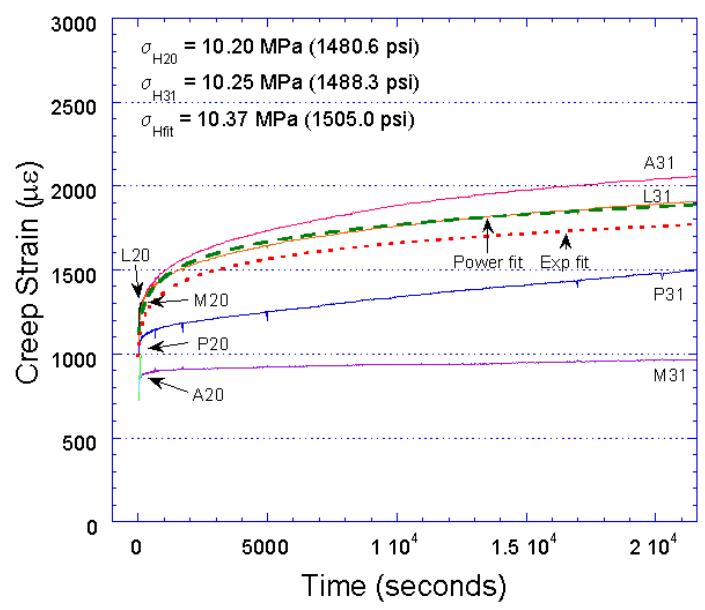

(E)

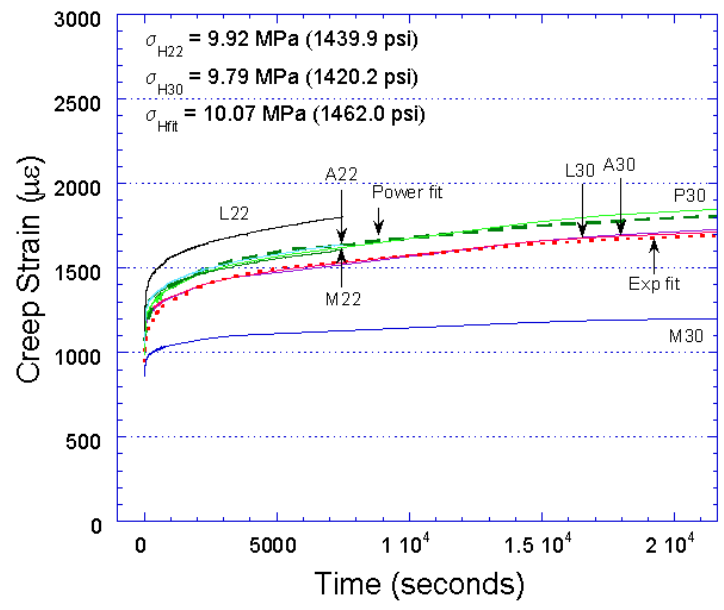

(D)

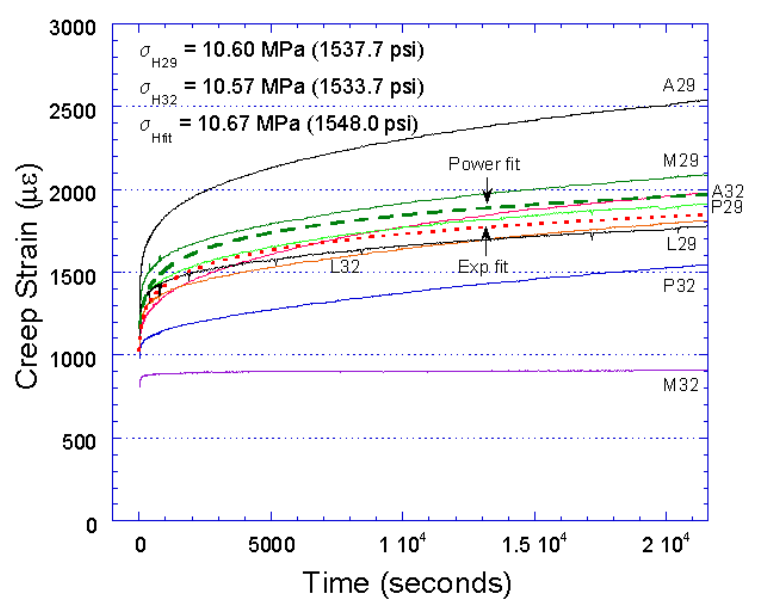

(F)

(G)

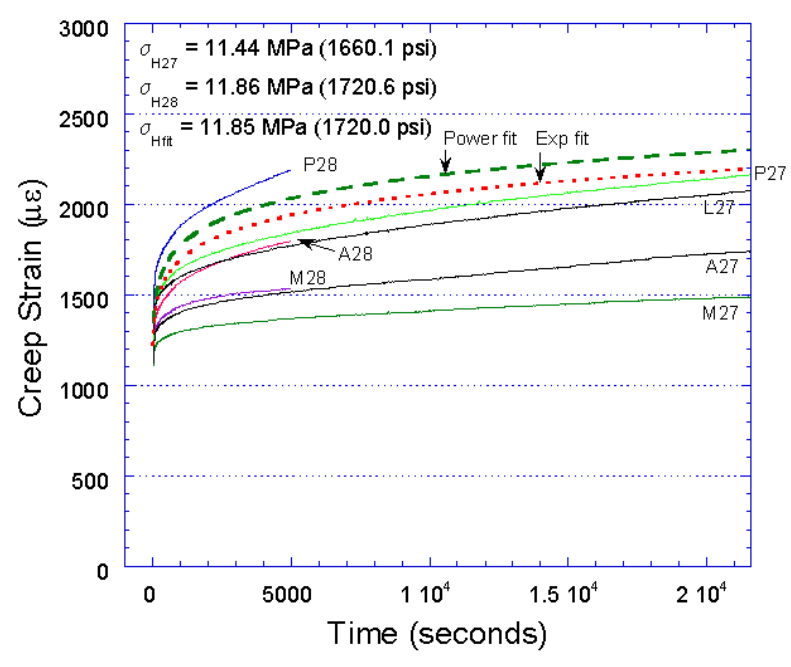

Figure 6.8 Measured creep strain and the power and exponential creep model for the different stress levels for the initial 6 hours $(2.16 \mathrm{E}+04$ seconds). A representative 
constant hoop stress was applied to the creep models which is close to the hoop stress level applied to the specimens. It is also important to note that the creep model was derived from measured strain from 15 specimens; it is not a curve fit of the strain data within a single plot.

\subsubsection{The Long-Term Creep Model}

A second creep model was developed to characterize the behavior over a time period of several days. The data used to derive this creep model were from specimens that failed after 6 hours of testing. The following were removed because the specimens fractured during the first 6 hours of constant pressure: Test \# 20, 22, 25, 28 and 33. A power law was used to model the behavior where $\varepsilon$ is strain in the units of microstrain, $\sigma$ is hoop stress in the units of $\mathrm{MPa}$ and $\mathrm{t}$ is time in seconds. The objective function converged to the following:

$$
\varepsilon=19.71 \sigma^{1.56} \mathrm{t}^{0.091}
$$

for all specimens and all quadrants combined (if $\sigma$ is in the units of psi then $\mathrm{p} 1=0.0084$ ). Additional power functions were determined for each quadrant:

$$
\begin{array}{ll}
\text { Posterior } & \varepsilon=24.89 \sigma^{1.50} \mathrm{t}^{0.079} \\
\text { Medial } & \varepsilon=32.81 \sigma^{1.31} \mathrm{t}^{0.079} \\
\text { Anterior } & \varepsilon=6.35 \sigma^{2.02} \mathrm{t}^{0.11} \\
\text { Lateral } & \varepsilon=46.09 \sigma^{1.23} \mathrm{t}^{0.086}
\end{array}
$$

(if $\sigma$ is in the units of psi then $p 1=0.014,0.047,0.00027$ and 0.10 for the posterior, medial, anterior and lateral quadrants, respectively).

The nonlinear curve fit option was also used to fit an exponential function from specimens that failed after 6 hours of testing:

$$
\varepsilon=140.69 \mathrm{e}^{(0.16 \sigma)} \mathrm{t}^{0.091}
$$

(if $\sigma$ is in the units of psi then $\mathrm{p} 2=0.0011$ ). Additional exponential functions were determined for each quadrant:

$$
\begin{array}{ll}
\text { Posterior } & \varepsilon=161.02 \mathrm{e}^{(0.16 \sigma)} \mathrm{t}^{0.079} \\
\text { Medial } & \varepsilon=168.43 \mathrm{e}^{(0.14 \sigma)} \mathrm{t}^{0.079} \\
\text { Anterior } & \varepsilon=84.91 \mathrm{e}^{(0.21 \sigma)} \mathrm{t}^{0.11}
\end{array}
$$




$$
\text { Lateral } \quad \varepsilon=214.63 \mathrm{e}^{(0.13 \sigma)} \mathrm{t}^{0.086}
$$

(if $\sigma$ is in the units of psi then $\mathrm{p} 2=0.0011,0.00095,0.0014$ and 0.00088 for the posterior, medial, anterior and lateral quadrants, respectively).

The power and exponential creep models were plotted at each of the seven hoop stress levels (corresponding target pressure levels) (Figure 6.9). These hoop stress levels were used in the creep functions to model the behavior at each stress level. The creep models can then be compared to the measured creep strain at approximately equal stress levels. Each specimen had a unique thickness and was pressurized to a slightly different pressure level for each test, thus resulting in different hoop stress levels (Table 6.2).

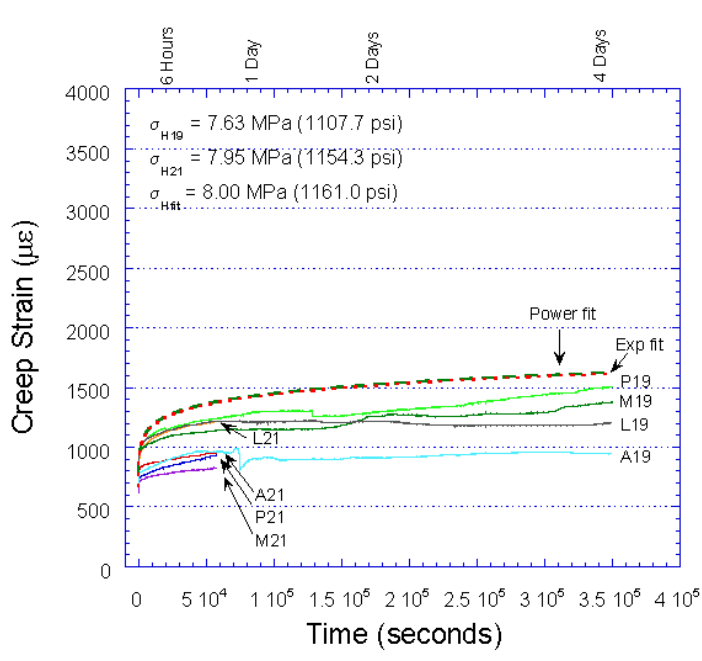

(A)

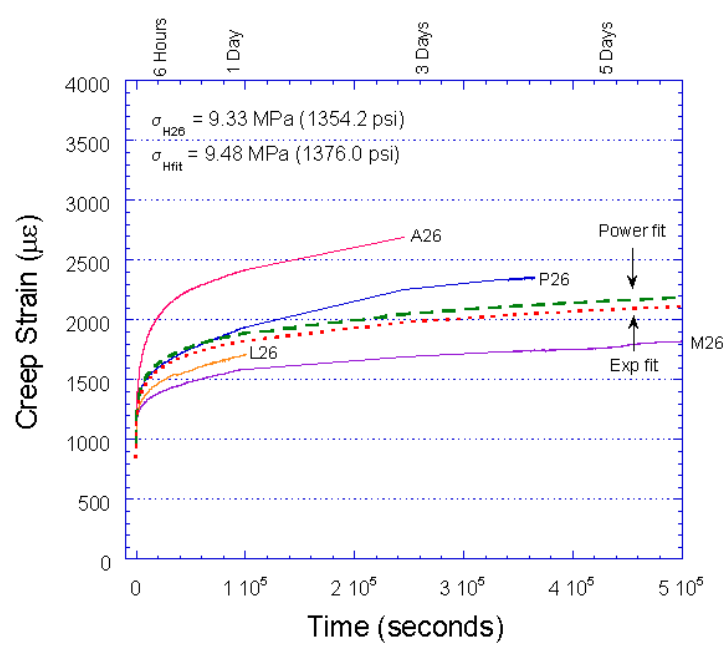

(C)

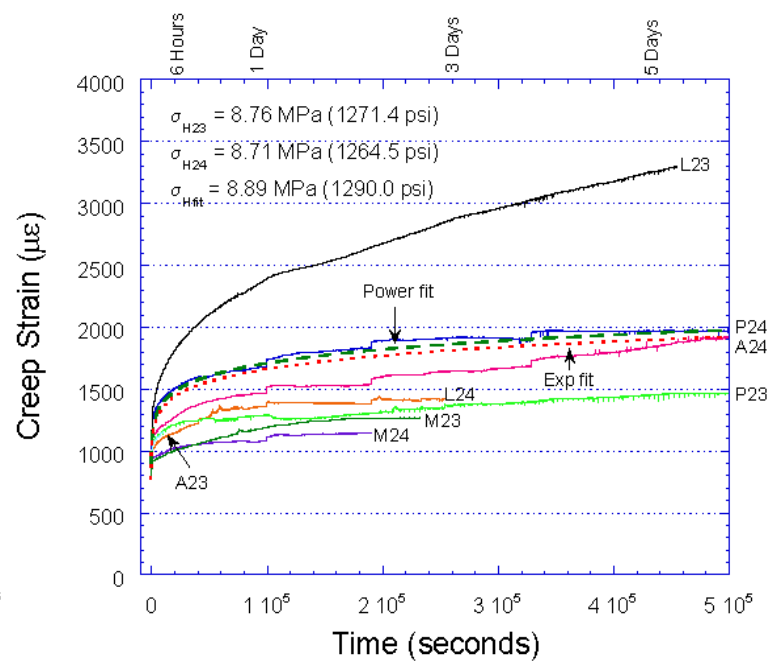

(B)

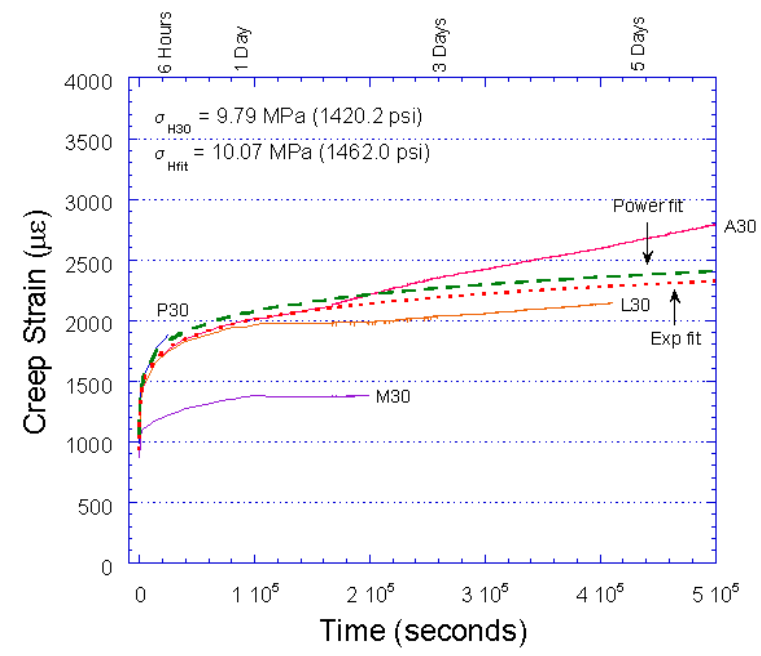

(D) 


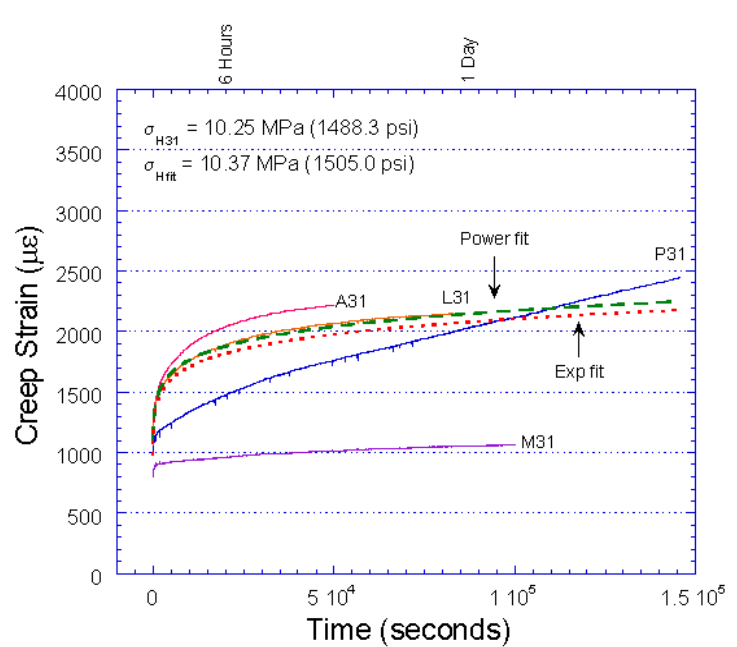

(E)

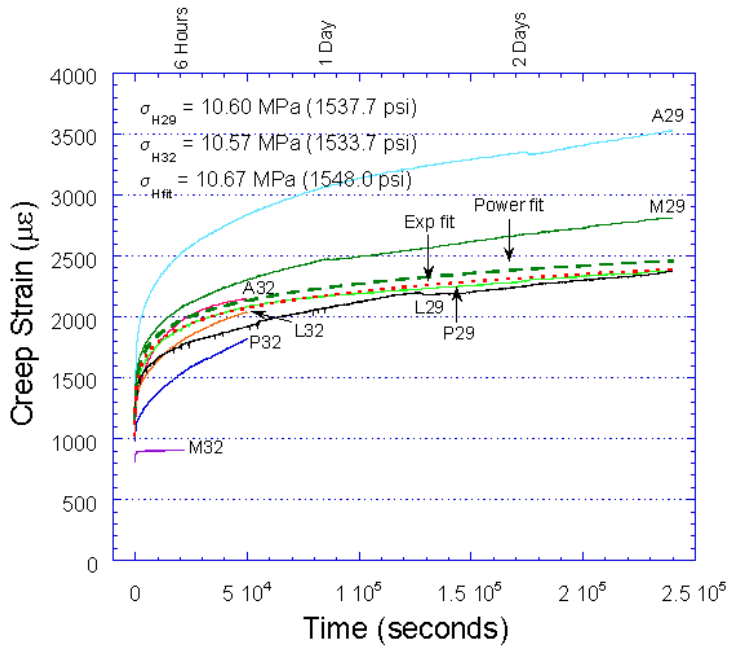

(F)

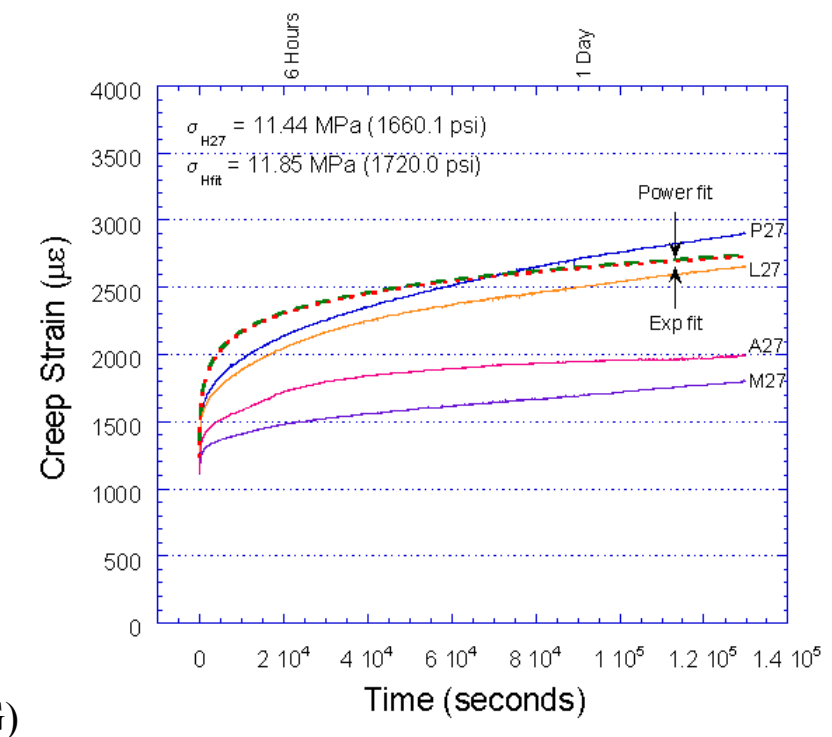

Figure 6.9 Measured creep strain and power and exponential creep models for the different stress levels for the duration of each test. A representative constant hoop stress was applied to the creep models which was close to the hoop stress level applied to the specimens. It is also important to note that the creep model was derived from measured strain from 10 specimens; it is not a curve fit of the strain data within a single plot. 
The power creep function that models the behavior during the first 6 hours of testing was chosen to examine the difference in the strain data per quadrant (Figure 6.10). The maximum, 11.85 MPa (1720 psi), minimum, 8.00 MPa (1161 psi) and medium, 10.07 MPa (1462 psi) stress levels were used in the creep model. The first 6 hours of creep was sufficient to observe the obvious difference in creep behavior among the quadrants, especially at the highest hoop stress level.

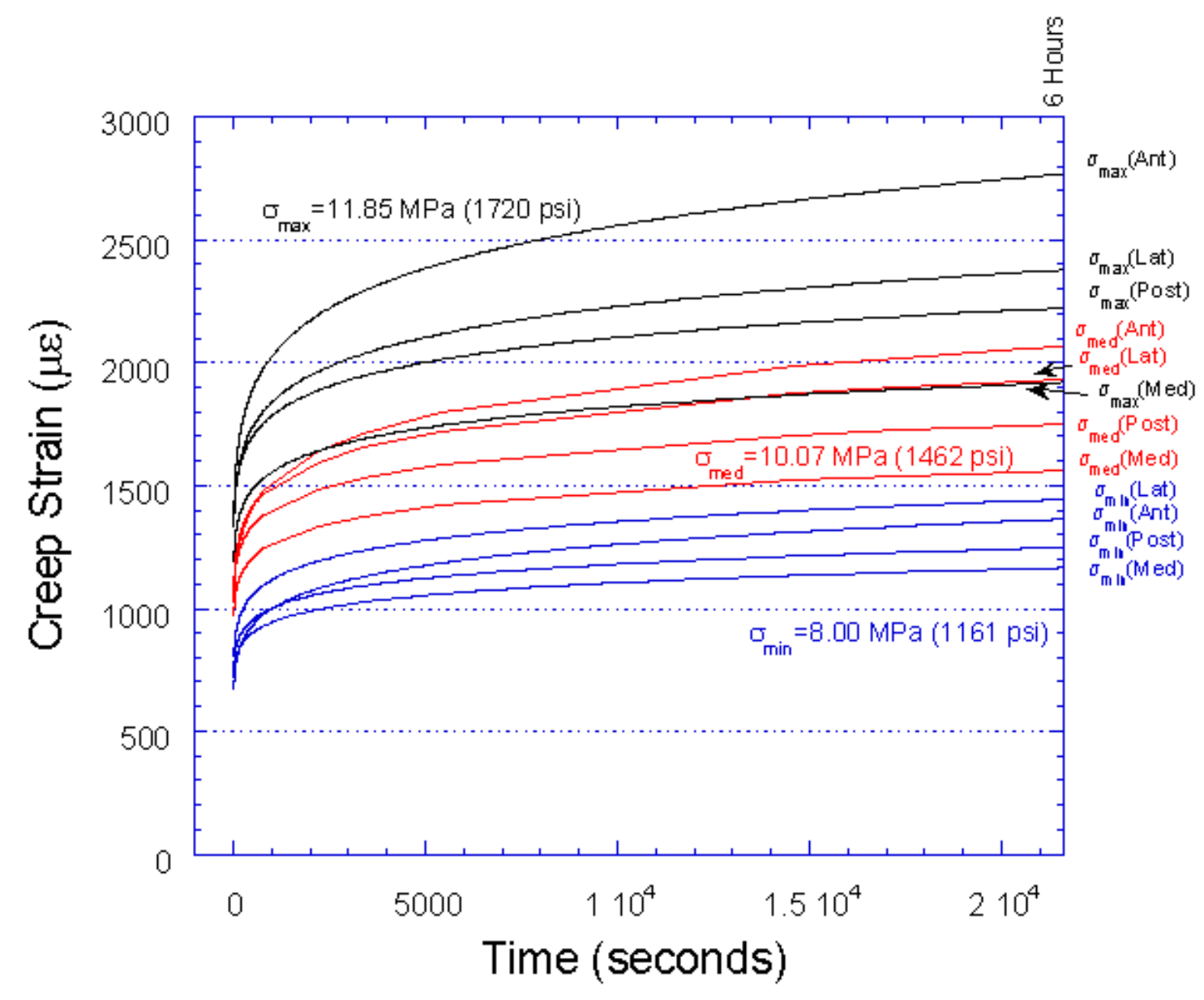

Figure 6.10 The power creep model for the behavior during the initial 6 hours for the maximum, minimum and medium stress levels. A difference in creep behavior was evident, especially at higher levels of hoop stress.

The power and exponential creep functions exhibited similar behavior (Figure 6.11). In general, the power function predicted higher strain than the exponential function. The functions deviated during the early, nonlinear, creep behavior within 5000 
seconds, and after this time the functions remained parallel. The difference between the two functions was at a minimum at the lowest hoop stress level.

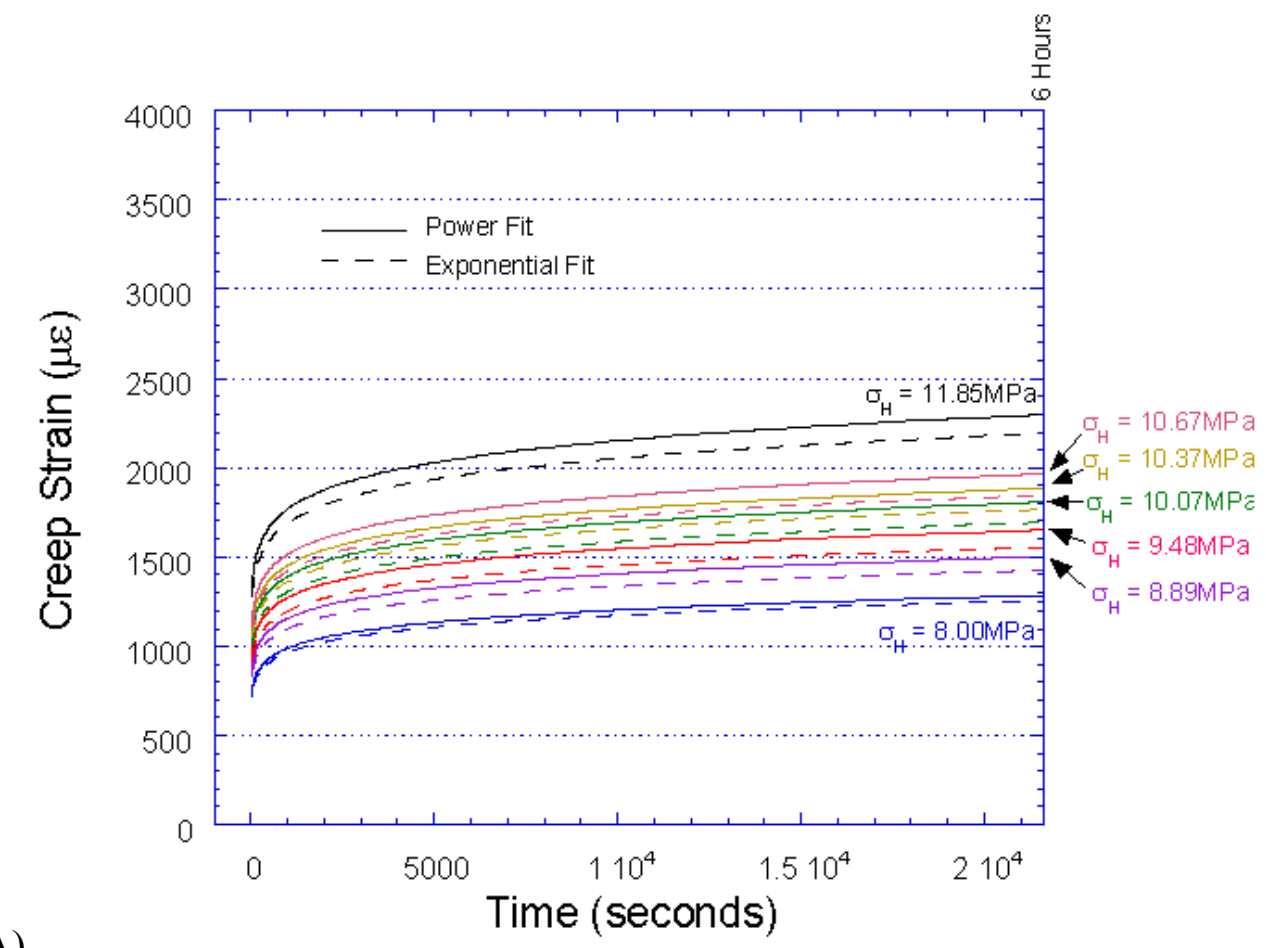

(A)

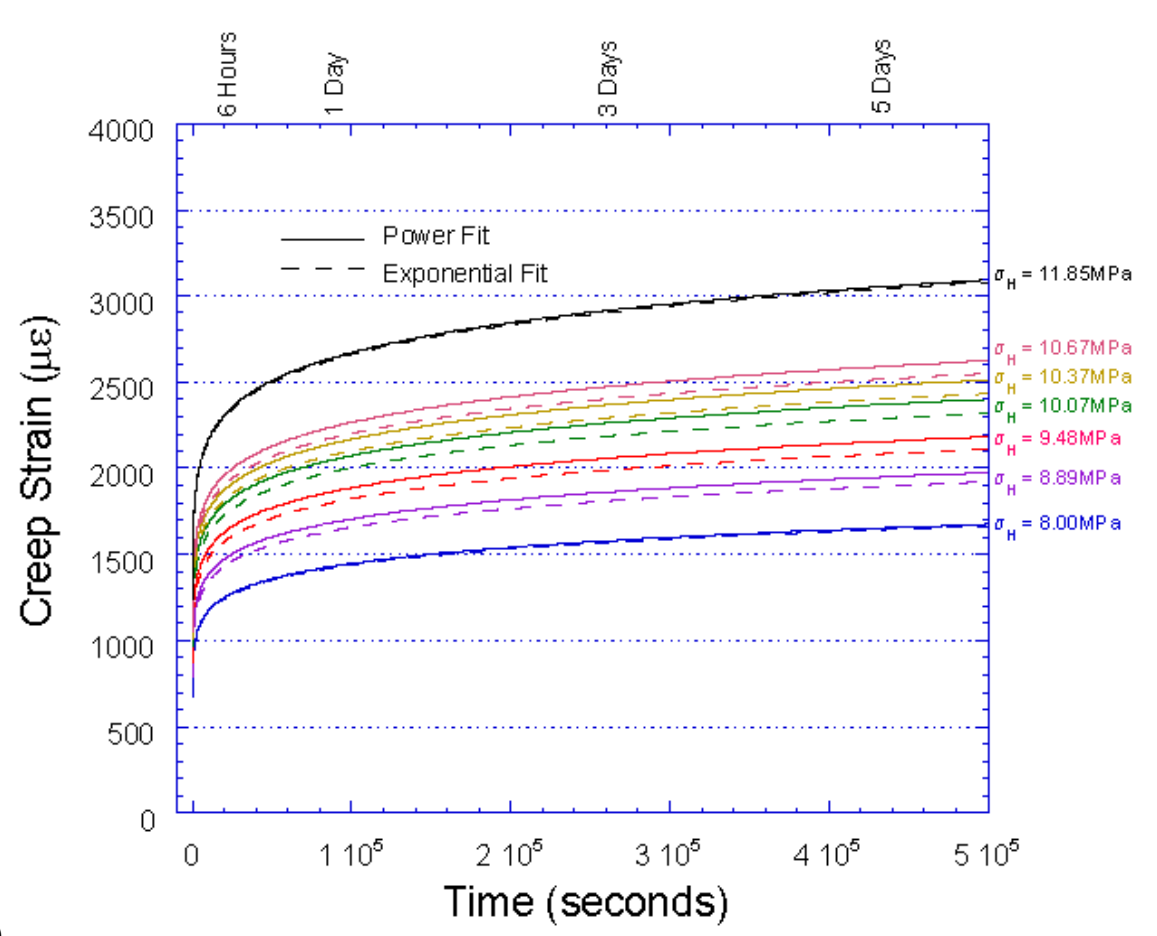

Figure 6.11 (A) The power and the exponential fits for the initial 6 hours and (B) for several days. Both models were very similar at all hoop stress levels. 
A linear regression of observed or experimental strain versus predicted strain from both the power and exponential functions was used to determine which function was better able to predict creep strain. A line with a slope of 1 represents the case when the predicted and observed strains are equal. For the general creep model including all the specimens, the observed versus the predicted strain yielded a line with a slope of 0.989 $\left(\mathrm{r}^{2}=0.540\right)$ and $0.989\left(\mathrm{r}^{2}=0.530\right)$ for the power and exponential functions, respectively. For the creep model of the behavior for the initial 6 hours, the observed versus the predicted strain yielded a line with a slope of $0.979\left(\mathrm{r}^{2}=0.652\right)$ and $0.981\left(\mathrm{r}^{2}=0.640\right)$ for the power and exponential functions, respectively. For the creep model of the behavior for longer than 6 hours, the observed versus the predicted strain yielded a line with a slope of $0.975\left(\mathrm{r}^{2}=0.689\right)$ and $0.976\left(\mathrm{r}^{2}=0.681\right)$ for the power and exponential functions, respectively. Based on these results it appears that either function was capable of predicting strain. 


\section{Multivariable Model of Long-Term Creep Strain}

Stepwise regression analysis was used for scanning variables for including in a regression model. A multivariable model using the least squares procedure was used for estimating long-term creep strain $(\mu \varepsilon)$ (Table 6.6). The control parameters examined were time (seconds), constant stress (stress) ( $\mathrm{MPa})$, location, gender, age, porosity (Po), osteon area (On.Ar), osteon density (On.Dn), single osteon area (s.On.Ar) (E-2), and initial stiffness (In.St) (GPa).

Table 6.6 Multivariable model of long-term creep strain.

\begin{tabular}{|ccc|}
\hline Creep strain $=-200.358$ In.St +0.00196 Time + 185.189 Stress -51.026 Location (M- \\
P) - 43.701 On.Ar +207.901 On.Dn +454.825 s.On.Ar -18.070 Gender -1.440 Age \\
+1.853 Po -649.42 & & $(6.38)$ \\
\hline In.St & $\mathrm{r}^{2}=0.318$ & $\mathrm{p}=0.0000$ \\
Time & $\mathrm{r}^{2}=0.544$ & $\mathrm{p}=0.0000$ \\
Stress & $\mathrm{r}^{2}=0.721$ & $\mathrm{p}=0.0000$ \\
Location (M-P) & $\mathrm{r}^{2}=0.726$ & $\mathrm{p}<0.0001$ \\
On.Ar & $\mathrm{r}^{2}=0.729$ & $\mathrm{p}=0.0000$ \\
On.Dn & $\mathrm{r}^{2}=0.730$ & $\mathrm{p}=0.0000$ \\
s.On.Ar & $\mathrm{r}^{2}=0.741$ & $\mathrm{p}=0.0000$ \\
Gender & $\mathrm{r}^{2}=0.742$ & $\mathrm{p}<0.0001$ \\
Age & $\mathrm{r}^{2}=0.743$ & $\mathrm{p}<0.0001$ \\
Po & $\mathrm{r}^{2}=0.744$ & $\mathrm{p}<0.0001$ \\
\hline Model & $\mathrm{r}^{2}=0.744$ & $\mathrm{p}<0.0001$ \\
\hline
\end{tabular}

All parameters were found suitable for the model most likely as the result of the large number of observations included in the model. Many of the parameters, osteon density, gender, age and porosity, however do not explain much of the variability in the model. This model was similar to the model of short-term creep strain (equation 5.5) where a positive relationship was found between stress, osteon density and porosity and an inverse relationship with initial stiffness. 


\subsection{Discussion}

Several limitations for these long-term tests were evident. Unfortunately shortcircuiting of the strain gages was unavoidable causing a loss of creep strain data for many tests. However, the nature of the creep strain was evident from the measured strain and the time of failure was always recorded. A minimum cortical thickness was needed to successfully machine the femoral cortex into a cylindrical specimen. This requirement precluded the use of femora with a thin cortex.

The time to failure relationship established here for normalized hoop stress was of limited use in itself. The relationship was only valid for predicting time to failure of bone specimens of the geometry used in this study as well as internally pressurized specimens, not necessarily for predicting intact femoral fractures. More specimens would improve the time to failure relationship within this pressure range. Increasing the number of pressure levels would also improve the ability to predict time to failure for a broader range of stress levels.

Vanscoy (1996) conducted creep tests of bone cylinders using a previous version of the testing equipment used in this study. The outer surface of the cylinders were left intact. The nonuniform geometry was thought to be the cause of compressive hoop strain on the surface as measured by strain gages. This compressive strain was encountered on the thickest cross-section of the bone specimen.

A similar phenomenon sometimes occurred during the third stage of creep in this study. A quadrant adjacent to a quadrant experiencing high tensile deformation underwent compressive strain (Figure 6.2). This phenomenon was thought to be similar to that reported by Vanscoy (1996). One quadrant was deforming at a faster rate than another causing the slower deforming quadrant to experience compressive strain. It is unknown as to what extent the interactions might be between the quadrants as they undergo creep strain. Perhaps the quadrant exhibiting the least creep strain was doing so because the adjacent quadrant was deforming more quickly and influencing the deformation.

The results of this study involve material properties of human cortical bone, not structural properties. The fact that compressive strains were not observed for the entire 
creep test as described by Vanscoy (1996) suggests that the compressive strains were in fact the result of the structural behavior as they predicted.

The range of internal pressures used for these long-term creep tests were chosen to span the pressure range of the threshold values of normalized hoop stress determined during the short-term tests. For the relationships between creep strain, creep rate, permanent strain and normalized hoop stress, thresholds were approximately 0.0011 (Chapter 5). At stress levels above this threshold, the specimens tended to fail between one and two days (Figure 6.4B and 6.5B). However at levels below the threshold, the specimens tended to take several days to fail. These results further suggest that there a change in behavior occurring near this level of normalized hoop stress, where stresses above the threshold caused shorter time to failure and for stresses below the threshold, time to failure was longer.

Extrapolating these results to the implant-bone environment after implantation is challenging. The results confirm the complexity of initial implant fixation which is dependent on the elastic response in bone to hold the implant until new bone grows. After the implant is inserted into the femur, a certain amount of force is needed between the implant and bone to maintain initial implant stability. Based on our short-term test results, hoop creep can occur at low stresses. The radial load may cause minor cracks at the time of surgery which may develop into fractures. When the bone creeps, the stem may subside deeper into the femur, reestablishing initial fixation and/or causing further creep in the bone. When the bone-implant environment reaches an equilibrium and the implant remains stable, the bone may grow to the stem and hold it in place and provide long term load transfer (Blaha, 1998). The deformation of cortical bone as seen in this study, may result in a loss of initial implant fixation or may cause fracture of the bone.

The results of this study suggest that creep strain does exist and can occur at low stress levels. Postoperative fractures due to press-fitted stems are occasionally unrecognized intraoperative fractures (Schwartz et al., 1989; Penenberg, 1998; Kelley, 1994). The tensile hoop and compressive radial stresses due to the internal pressure were greater along the inner surface of the cylinder then the outer surface. These high stresses could induce creep fracture along the inner surface before the outer surface. The results suggest that the creep fracture observed in this study may account for the appearance of 
fractures postoperatively that had previously been assumed to be unrecognized intraoperative fractures.

\subsubsection{Time to Failure}

A time to failure relationship was established where specimens experiencing higher levels of hoop stress failed at shorter lengths of time than did specimens at low hoop stress. This relationship can not be directly applied to the intact femur due to the thinner specimen thickness and the fact that the periosteum had been removed. This time to failure relationship however was examined to better understand the creep behavior at and around the value of normalized hoop stress that may serve as a threshold between linear and nonlinear behavior.

The average initial hoop stiffness from all the quadrants of all the specimens was 9.71 GPa indicating that the stiffness of the bone specimens used for these tests was similar to the bone specimens used in the short-term tests. This suggests that the normalized hoop stress in the short term tests was comparable to the normalized hoop stress used in the time to failure relationship.

Figure $6.4 \mathrm{~A}$ is a linear-linear plot of a non-significant relationship between normalized hoop stress and time to failure with a logarithmic fit. Based on this curve, there appeared to be a large increase in time to failure as the normalized hoop stress decreased. This data suggests that there is a change in the length of time to failure at a normalized hoop stress of approximately 0.0011 , approximately the threshold determined for the creep strain, creep rate and permanent strain behavior from the short term tests. After two specimens were removed, the time to failure relationship was significant and the logarithmic fit continues to show a large increase in time to failure with decreasing normalized hoop stress.

A power law relationship was used to describe the relationship of time to failure and normalized hoop stress for longitudinal oriented bone specimens as reported by Caler and Carter (1989). The normalized longitudinal stress level range used in their study was 0.002 to 0.006 , which is higher than the normalized hoop stress used in this study. The time to failure relationships between normalized longitudinal stress and normalized hoop stress were compared. At any normalized stress level, creep failure will occur sooner in 
the hoop direction. This was expected since it is well known that bone has adapted its microstructure to resist loads in the longitudinal direction, which are more likely to occur in vivo.

All specimens fractured eventually except one for the stress levels used in our study. The stress levels used were within the range of stress levels in the previous shortterm tests to determine a threshold. Based on these creep tests it appears that the bone specimens tested at stress levels around the stress threshold would fracture given enough time. This suggests that the threshold determined from the short-term tests is not a useful factor for determining a sufficiently low stress to avoid failure.

\subsubsection{The Creep Model}

These results show that cortical bone exhibits a hoop creep response that was stress level dependent. A power and exponential function were used to derive a creep model to characterize the creep strain as a function of time and hoop stress. The creep data was used to derive a function for the general case where all the specimens were included accounting for the high variability in human bone. Two other creep models were derived to characterize the behavior during the initial six hours of testing and to characterize the behavior for several days.

Chwirut (1984) studied compressive creep deformation of acrylic bone cements and used two stress functions for modeling strain behavior as a function of stress and time. A power law stress function yielded information regarding the linearity of the viscoelastic behavior but was not suitable for modeling behavior at low-stress levels. The second model was an exponential stress function which did not model zero strain when stress was zero, however mid to high stress levels were represented well. In general, for the acrylic bone cements, the exponential function modeled the creep behavior better than the power function however the predictions of strain at low levels of stress were invalid.

Both the power and exponential functions fit the creep strain data well overall as indicated by the slope of the linear regression being nearly one for all comparisons. This also supports either the power or the exponential function may be used to predict hoop strain. The functions did not follow the data at very early times, during the initial 30 seconds of the test where the observed creep strain was very nonlinear. For the creep 
model derived for the initial 6 hours of testing, the power and exponential functions did not appear to follow the data well for approximately the first 5000 seconds at low stress levels (Figure 6.8A, B and C). At higher stress levels, the functions followed the data closely. Both functions were parallel from the beginning of the creep tests at various stress levels. Evidence of their similarity was the fact that the time exponents were the same between the functions for each of the three pairs of creep models and for the relationship at each quadrant.

The creep models derived from the experimental creep strain may be directly applied to finite element models to study the effects of creep behavior in both cemented and cementless implant models. Using the creep models for each individual quadrant is recommended for future modeling of hoop strain based on the variation of the creep strain data for each quadrant for the range of hoop stress. Two creep models were derived from this data for characterizing the creep behavior for short and long durations and may be selected based upon the modelers preference. The unique shape and loading environment resulted in hoop stiffness values to be determined rather than values determined from rectangular specimens. The hoop stiffness values are important when bone is assumed to be an orthotropic material. Better computer models, which include more types of bone behavior, will ultimately benefit patients. 


\section{CHAPTER 7}

FAILURE MECHANISMS IN CYLINDRICAL CORTICAL BONE SPECIMENS

DUE TO RADIAL LOADING

(Brown et al., 2000b, BED ASME)

\subsection{Introduction}

Damage in cortical bone may compromise the initial fixation of the implant. Microdamage changes mechanical properties, such as stiffness, in cortical bone (Carter and Hayes, 1977; Fondrk et al., 1988) and has been implicated in contributing to loosening of joint prostheses (Frost, 1986; Martell et al., 1993). Microdamage may occur during preparation of the femoral canal and during insertion of the final sized stem resulting in gross fracture of the host bone (Fitzgerald et al., 1988; Schwartz et al., 1989; Kelley, 1994).

Fondrk et al. (1988) found that creep strain is produced at a constant rate and therefore needs time to accumulate. Applying constant load to bone may cause unrecoverable deformation and ultimately lead to creep failure in longitudinally oriented bone specimens (Carter and Caler, 1983; Caler and Carter, 1989; Fondrk et al., 1988). Creep-fracture data indicates differences in time-dependent damage accumulation behavior for tension and compression that is dependent on load magnitude and possibly also different fracture mechanisms (Caler and Carter, 1989). Caler and Carter (1989) showed different fracture surfaces depending on the magnitude of load and also mode of loading, compression or tension.

Characterizing damage resulting from radial loading would enhance our understanding of damage mechanisms associated with time-dependent damage responsible for viscoelastic failure of press-fit total hip arthroplasty. The objective was to assess damage morphology to determine the damage mechanisms in cortical bone due to a radial load as would be applied by a press-fit implant.

\subsection{Materials and Methods}

One left and one right femur with non-osteoporotic bone quality were obtained from an 86-year-old male cadaver. A bulk section of each femoral mid-diaphysis was wet 
machined to obtain four right circular cylinders $(n=4)$ each with a thickness of $2.4 \mathrm{~mm}$. A test fixture applied constant pressure to the inner surface of the specimen in a saline solution bath at $37^{\circ} \mathrm{C}$ until failure (Brown et al., 2000a). This device idealizes the radial forces immediately following the insertion of a maximum fit and fill press-fit implant. Specimens were loaded at a constant internal pressure between 1 and $2 \mathrm{MPa}$ until failure. The internal pressure used for this study resulted in hoop strains that were within a range of reported values for press-fitting a stem into a femur (Jasty et al., 1993). Two additional specimens from a 45-year-old male cadaver were also wet-machined. One served as a control $(n=1)$ and was not tested. The other was tested and exhibited extensive creep strain but was not tested to failure $(n=1)$.

After testing, the specimens were stained en bloc with $1 \%$ basic fuchsin dissolved in ascending concentrations of ethanol (Burr and Hooser, 1995) and sectioned for histological analysis. Bulk staining techniques distinguish between artifactual damage caused by section preparation from in-vivo damage due to loading. Sections, $200 \mu \mathrm{m}$ thick, were removed from each quadrant with a low speed saw (Buehler, Ltd., Lake Bluff, IL) and mounted on glass slides for microscopic observations. Fields, $651 \mu \mathrm{m}$ by $651 \mu \mathrm{m}$, were randomly chosen from periosteal and endosteal surfaces of each section (Figure 7.1), and a Laser Scanning Confocal Microscope (Ziess LSM-510) was used to examine damage morphology. An argon ion laser with a wavelength of $488 \mathrm{~nm}$ was used, however, basic fuchsin excites at $560 \mathrm{~nm}$. Despite this difference, the strained microcracks were clearly observed.

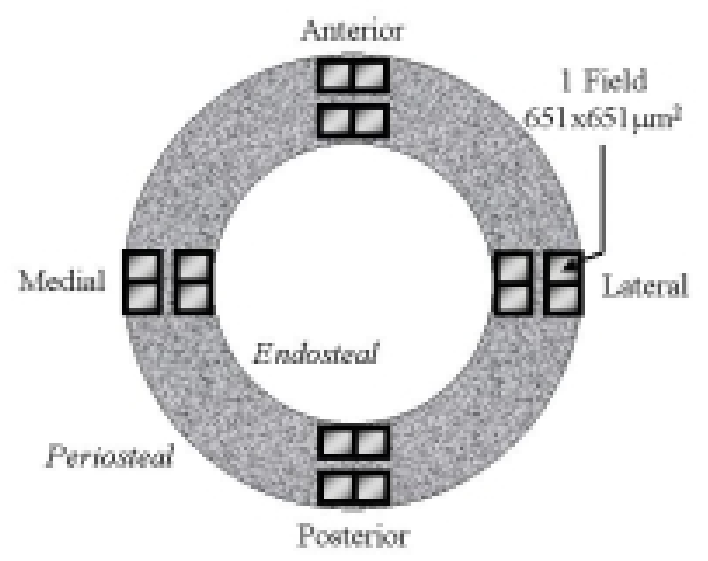

Figure 7.1 Four fields, $651 \mu \mathrm{m}$ by $651 \mu \mathrm{m}$, were used to measure microcracks at each quadrant. 
The number of cracks in a field, microdamage density $\left(\# / \mathrm{mm}^{2}\right)$, and the total length of all cracks within a field, microdamage surface density $\left(\mu \mathrm{m} / \mathrm{mm}^{2}\right)$, were measured at each anatomical region (anterior $(\mathrm{A})$, medial $(\mathrm{M})$, posterior $(\mathrm{P})$, lateral $(\mathrm{L})$ ), and the crack orientation was noted. Cracks oriented $\pm 15^{\circ}$ parallel to the direction of the radial load were recorded as radial cracks. Cracks oriented $\pm 15^{\circ}$ perpendicular to the applied load were recorded as hoop cracks. Cracks greater than $15 \mu \mathrm{m}$ and clearly distinct were considered discrete microcracks. Based on previous work (Norman and Wang, 1997), microdamage was classified (Table 7.1) using a modified protocol as the following different types (Figure 7.2).

Table 7.1 Microdamage type including description.

\begin{tabular}{|c|l|}
\hline $\begin{array}{c}\text { Damage } \\
\text { type }\end{array}$ & \multicolumn{1}{|c|}{ Damage Description (abbreviation) } \\
\hline 1 & microcracks in the matrix (m) \\
\hline 2 & microcracks extending from matrix to the cement line (mc) \\
\hline 3 & microcracks along the cement line (c) \\
\hline 4 & microcracks along an individual lamella, intralamellar microcracks (1) \\
\hline 5 & $\begin{array}{l}\text { microcracks that intersect several lamellae, interlamellar microcracks, } \\
\text { approaching but not reaching either the Haversian canal or cement line }\end{array}$ \\
\hline 6 & microcracks from the Haversian canal to a lamella (ql) \\
\hline 7 & $\begin{array}{l}\text { microcracks that propagate from the matrix to a lamella, across the cement } \\
\text { line (ml) }\end{array}$ \\
\hline 8 & microcracks from the matrix to the Haversian canal (mq) \\
\hline 9 & $\begin{array}{l}\text { microcracks that propagate from the cement line of one osteon to the cement } \\
\text { line of a different osteon (cc) }\end{array}$ \\
\hline 10 & microcracks from the cement line to the Haversian canal (cq) \\
\hline 11 & microcracks from the cement line to a lamella (cl) \\
\hline 12 & $\begin{array}{l}\text { microcracks that propagate from the Haversian canal of one osteon to the } \\
\text { Haversian canal of a different osteon (qq) }\end{array}$ \\
\hline 13 & $\begin{array}{l}\text { microcracks that propagate from a lamella of one osteon to a lamella of a } \\
\text { different osteon (1l) }\end{array}$ \\
\hline 14 & $\begin{array}{l}\text { microcracks that propagate from the cement line of one osteon, through the } \\
\text { matrix and ends at a lamella of a different osteon (cml) }\end{array}$ \\
\hline
\end{tabular}




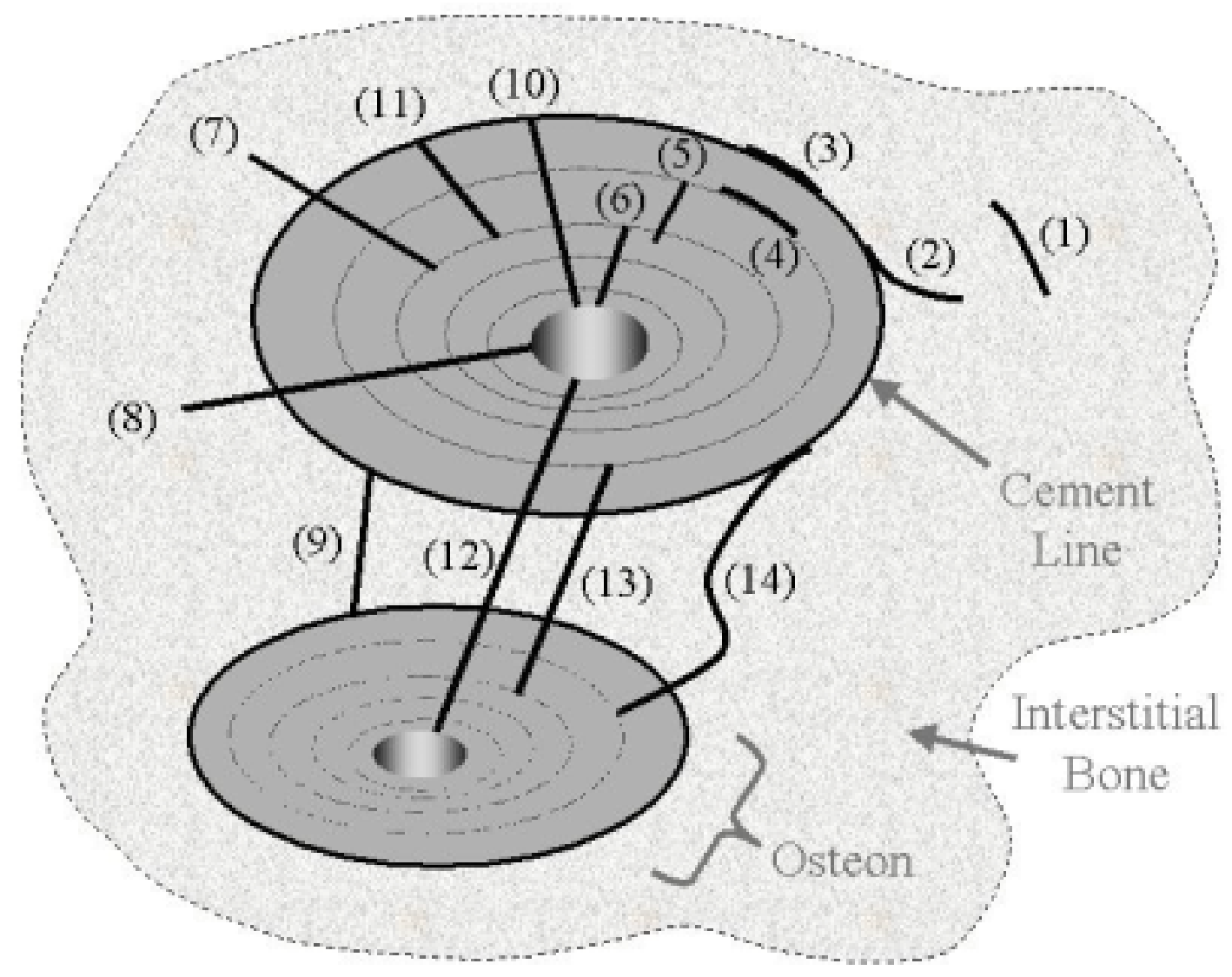

Figure 7.2 Different types of microdamage within and around an osteon in human cortical bone. 


\subsection{Results}

The four specimens that were tested until fracture had similar results to that of the one specimen that was not tested to fracture. This was most likely due to the rigorous testing that the specimen experienced. These five specimens were therefore grouped together $(n=5)$. Surface observations revealed microcracking patterns in endosteal and periosteal regions. In the five tested specimens more microcracks were observed near the endosteal surface, $12.33 \# / \mathrm{mm}^{2}$, than near the periosteal surface, $7.20 \# / \mathrm{mm}^{2}$ (Figure 7.3).

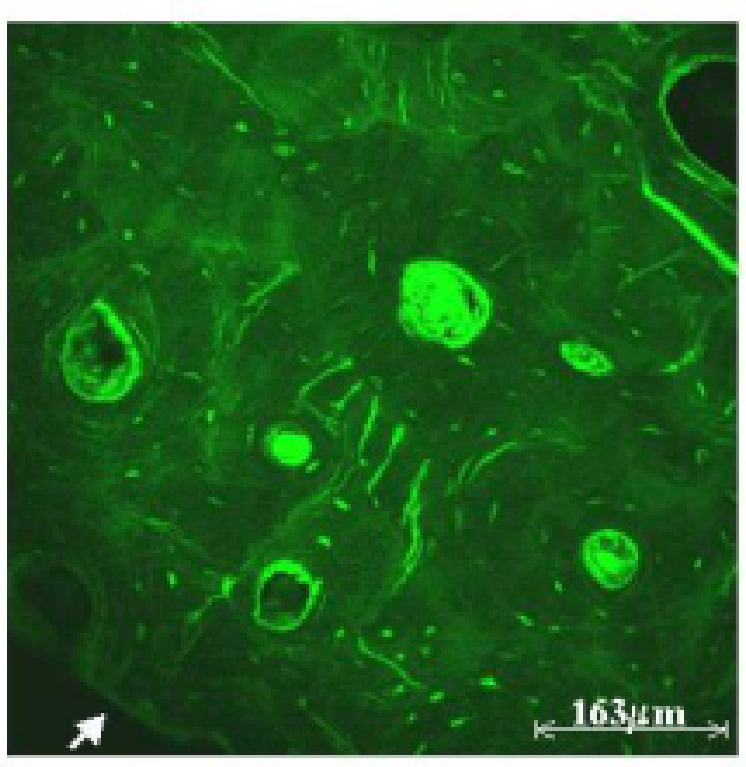

Endosteal surface

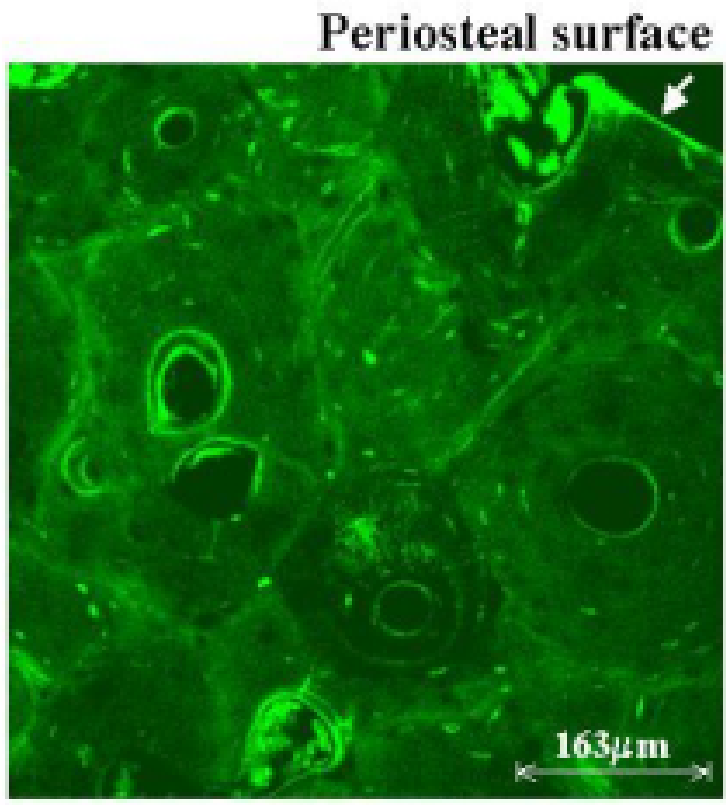

(B)

Figure 7.3 (A) Microcracks at the endosteal surface and (B) at the periosteal surface. A greater number of cracks were observed at the endosteal surface. Images are 651 x 651 $\mu \mathrm{m}^{2}$. 
The average microdamage density for the tested specimens, $9.76 \# / \mathrm{mm}^{2}$, was greater than the control, $6.05 \# / \mathrm{mm}^{2}$. This trend was also true for the average microdamage surface density, $722.13 \mu \mathrm{m} / \mathrm{mm}^{2}$ for the tested specimens and 519.85 $\mu \mathrm{m} / \mathrm{mm}^{2}$ for the control (Figure 7.4). Schaffler et al. (1995) found a crack density range of 0.5 to $2 \# / \mathrm{mm}^{2}$ for an age range from 60 to 75 years for in-vivo bone. Using their exponential relationship of damage density and age, their curve predicts a value near 6 $\# / \mathrm{mm}^{2}$ at our age. Norman and Wang (1997) report a lower value of approximately 0.25 $\# / \mathrm{mm}^{2}$ at an age of 86 years.

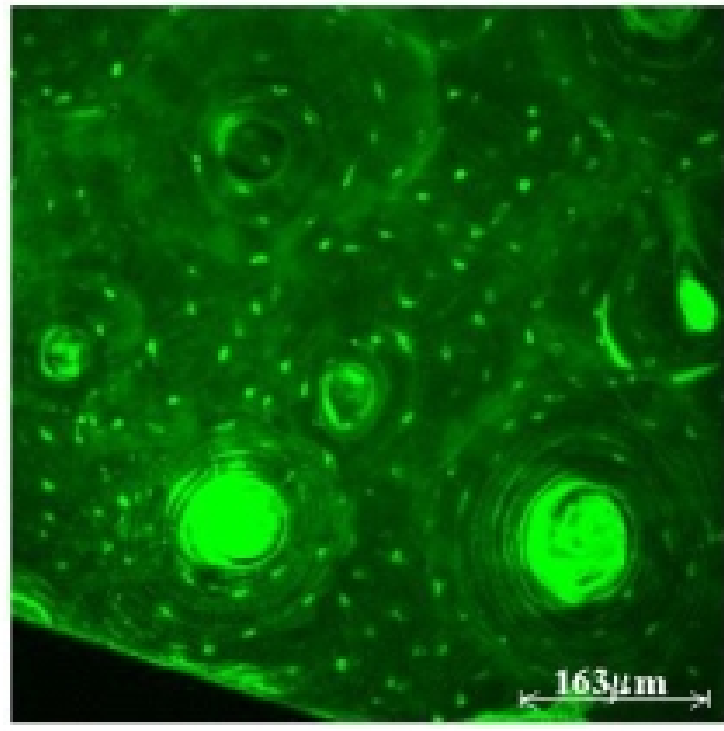

\section{Control}

(A)

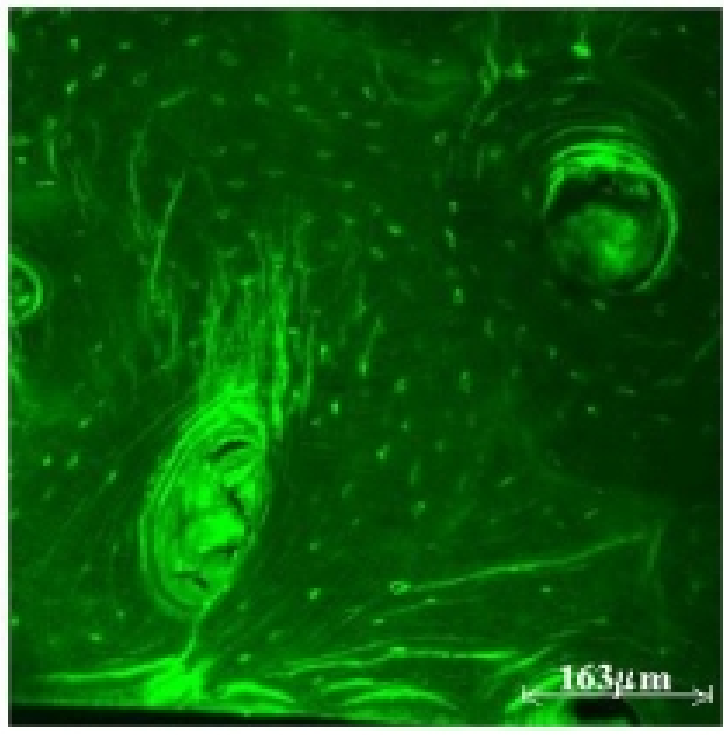

Tested

(B)

Figure 7.4 Images at the endosteal surface of the (A) untested, control, specimen and (B) a tested specimen. Microdamage density and microdamage surface density were greater for the tested specimens than for the control.

In the tested specimens, radial microcracks, parallel to the direction of the applied load, occurred more frequently, $4.78 \mathrm{\#} / \mathrm{mm}^{2}$, than cracks oriented in the hoop direction, $2.86 \# / \mathrm{mm}^{2}$ (Figure $7.5,7.6$ ) and oblique cracks, $2.12 \# / \mathrm{mm}^{2}$. A significant difference was found $(\mathrm{p}<0.05)$ between the crack density of radial and hoop oriented cracks. When the crack density between radial and hoop cracks was grouped by anatomical region, the 
radial crack density of each quadrant was greater than the hoop crack density but the comparison was only significant for the anterior quadrant.

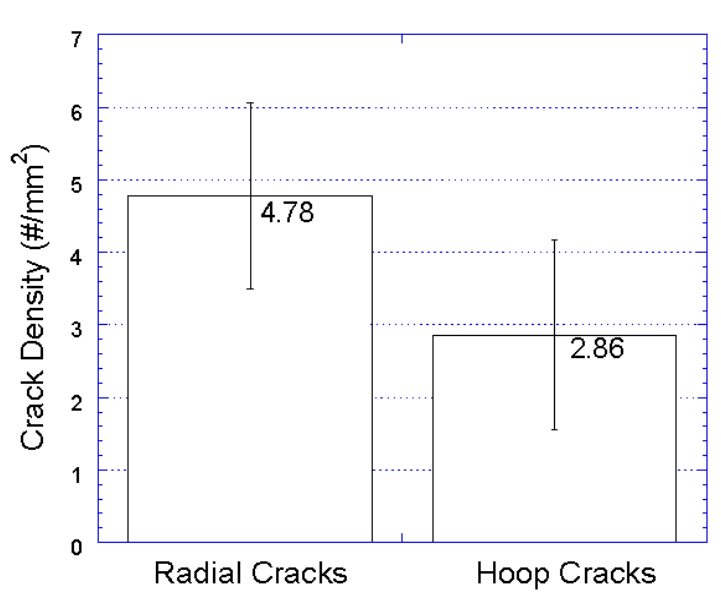

(A)

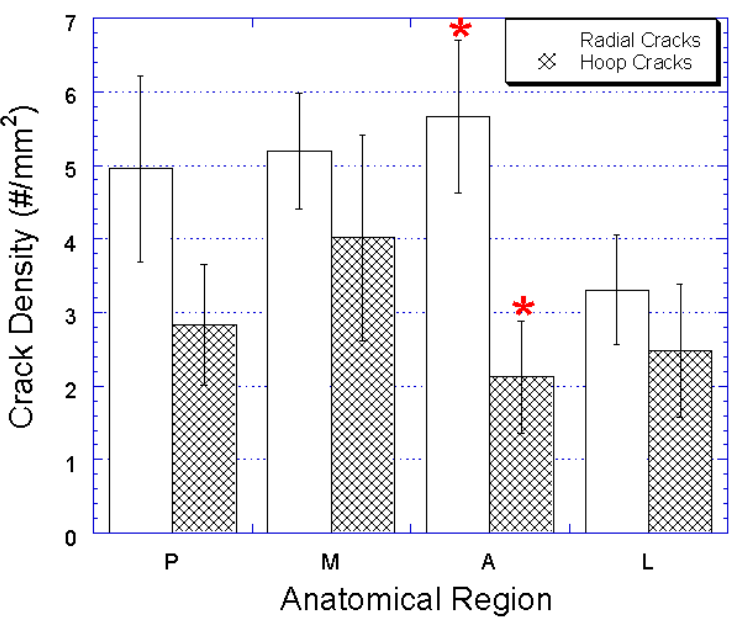

(B)

Figure 7.5 (A) Radial crack density was significantly greater than hoop crack density for the tested specimens $(\mathrm{p}<0.05)$ and $(B)$ crack density versus anatomical region for the five tested specimens, where the anterior quadrant has a significantly greater radial crack density than hoop crack density $(\mathrm{p}<0.05)$.

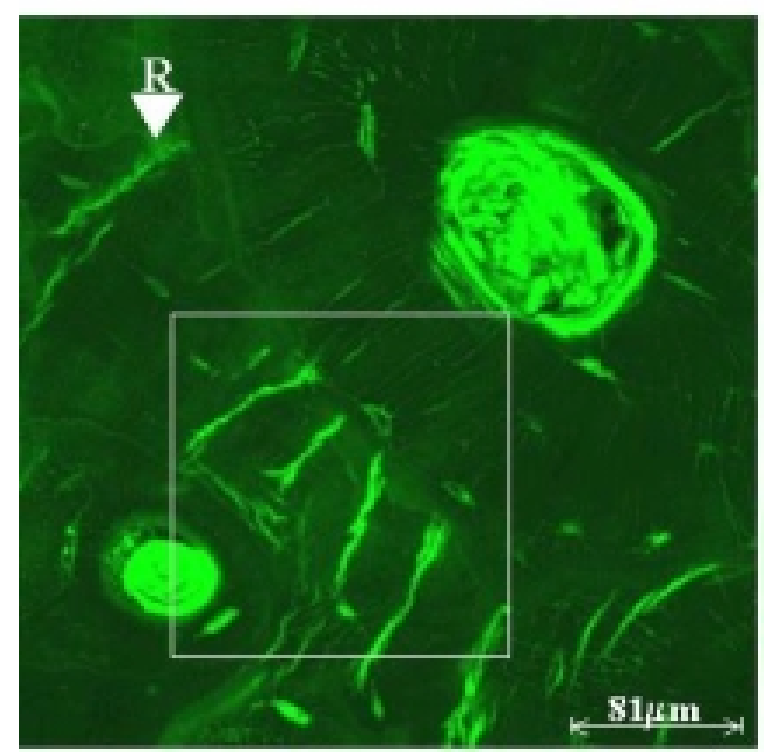

(A)

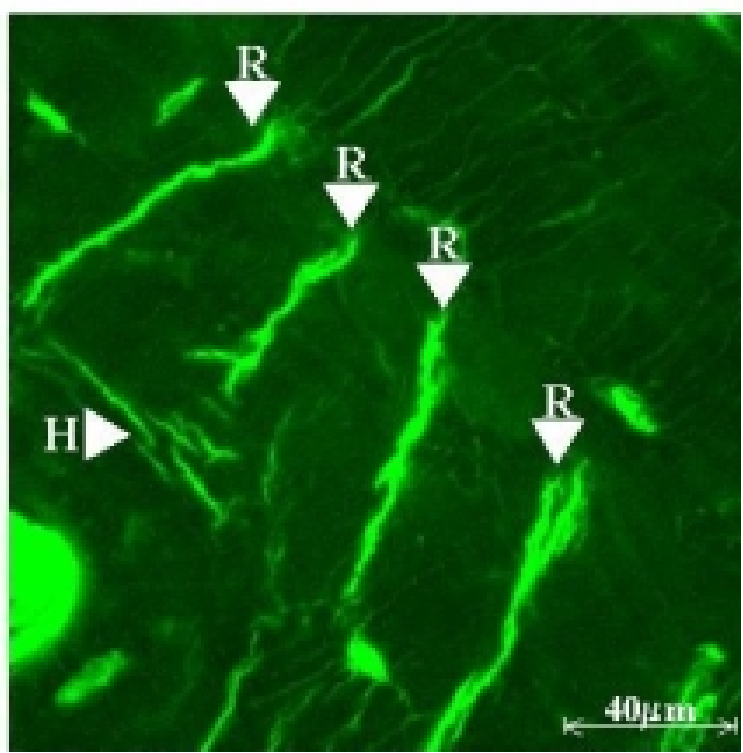

(B)

Figure 7.6 More radial cracks were found compared to hoop oriented cracks. (A) Microcracks are extending between cement lines of two osteons and into the matrix of cortical bone. Cracks along the cement line are visible around the bottom left osteon 
(325.5 $\times 325.5 \mu \mathrm{m}^{2}$ ); (B) magnified image taken from within the white box of (A), $\mathrm{H}$ indicates a microcrack in the hoop direction and $\mathrm{R}$ indicates a crack in the radial direction $\left(162.75 \times 162.75 \mu \mathrm{m}^{2}\right)$.

In the tested specimens, microdamage was grouped into one of 14 types depending on its microstructural location. Microdamage in the matrix, extending from the matrix to a cement line, or in the matrix between the cement lines of two different osteons, types 1, 2 and 9 respectively, were found to occur with the highest frequency and together account for $74 \%$ of all damage observed (Figure 7.7).

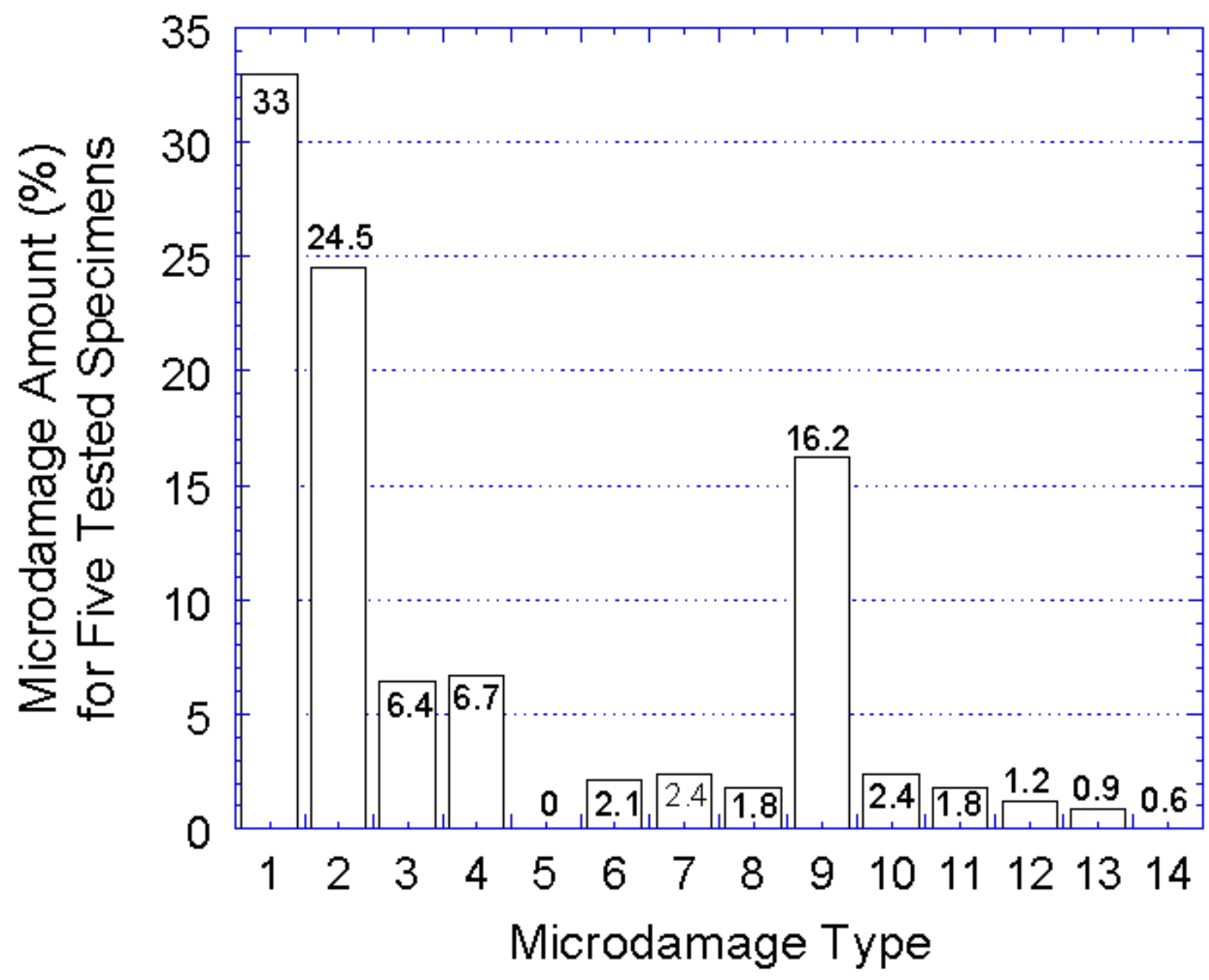

Figure 7.7 The percentages of the different types of microdamage for the five tested specimens.

In an attempt to simplify these results, all the microdamage was regrouped into only four groups: (1) cracks in the interstitial matrix or interstitial bone into a cement 
line, (2) cracks entirely within the cement line, (3) cracks contained within a Haversian system, and (4) all other cracks including cracks that pass entirely through a cement line. According to this regrouping, $74 \%$ of the damage occurs within the matrix (previously types 1, 2 and 9), $6 \%$ of the cracks are along a cement line (previously type 3 ), $13 \%$ is contained entirely within a Haversian system (previously types 4, 5, 6, 10 and 11), and $7 \%$ pass through a cement line (previously types 7, 8, 12, 13 and 14) (Figure 7.8).

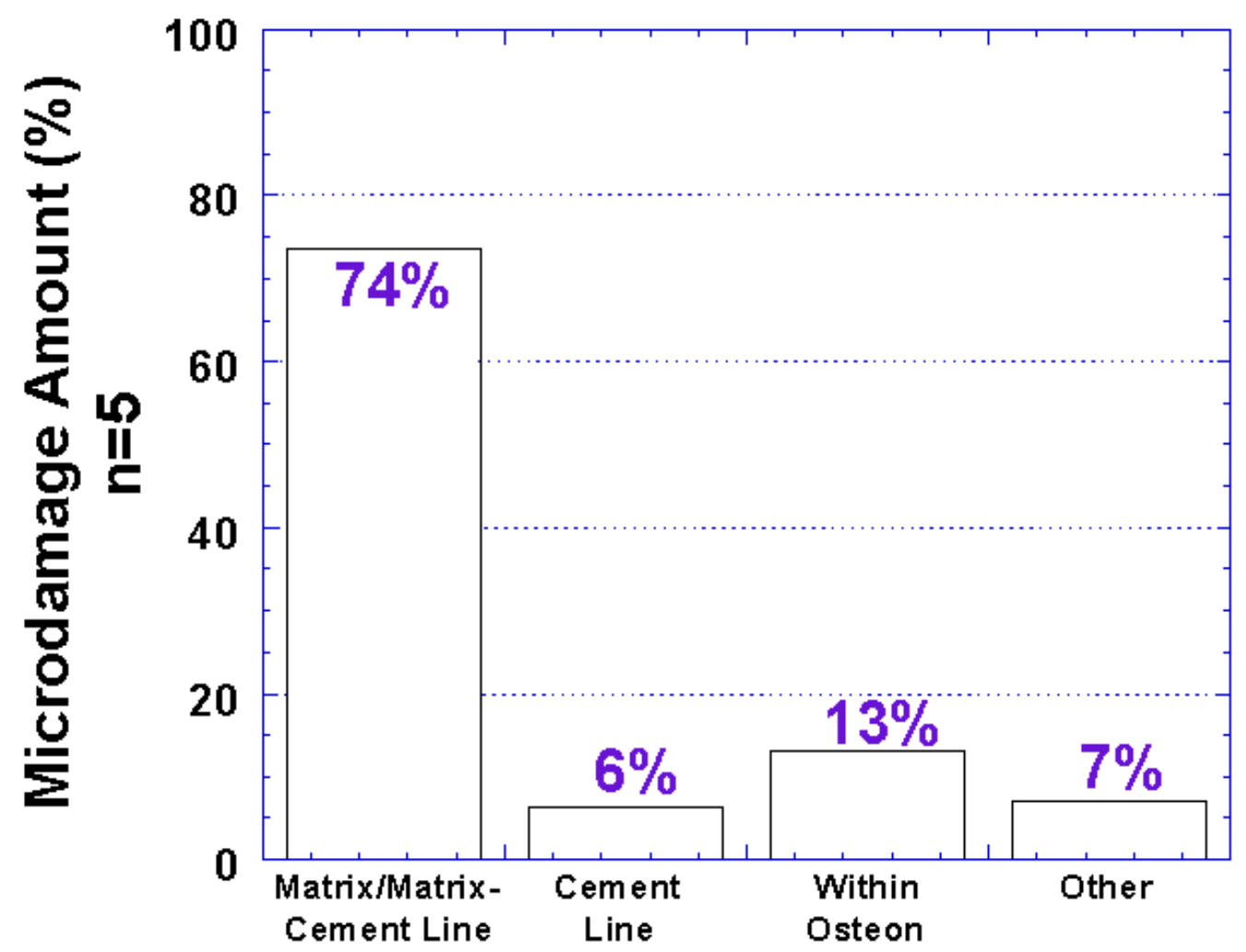

Figure 7.8 Microdamage in the matrix or in the matrix and along a cement line was the most frequent form of damage.

\subsection{Discussion}

For an internally loaded thick walled elastic cylinder, with this geometry and a thickness of $2.4 \mathrm{~mm}$, a large tensile hoop stress exists near the inner surface, approximately 4.5 times the internal pressure $\left(\mathrm{P}_{\mathrm{i}}\right)$, which decreases towards the outer surface, to about 3.5 $\mathrm{P}_{\mathrm{i}}$. A compressive radial stress also exists at the inner surface equal to the magnitude of the internal pressure and approaches zero towards the outer surface (Figure 7.9). In summary, at the inner surface of the specimen a combination of large 
hoop and smaller radial stress exists while at the outer surface only hoop stress exists. The combination of higher stress at the inner surface supports the observation that, in general, more microcracks occur at the endosteal surface, as compared to the outer surface.

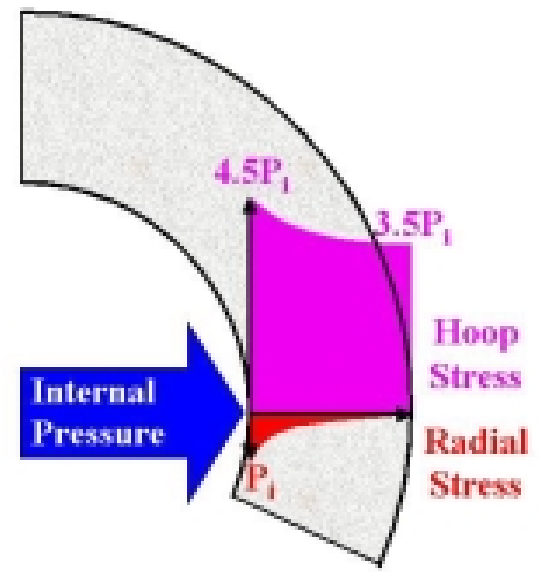

Figure 7.9 The internal pressure results in a combination of large tensile hoop stress and a smaller radial stress at the inner surface. Only hoop stress exists at the outer surface.

More cracks were observed to be oriented in the radial direction than in the hoop direction. Based on this observation it is suggested that the tensile hoop and compressive radial stresses serve to open microcracks in the radial direction (Figure 7.10).

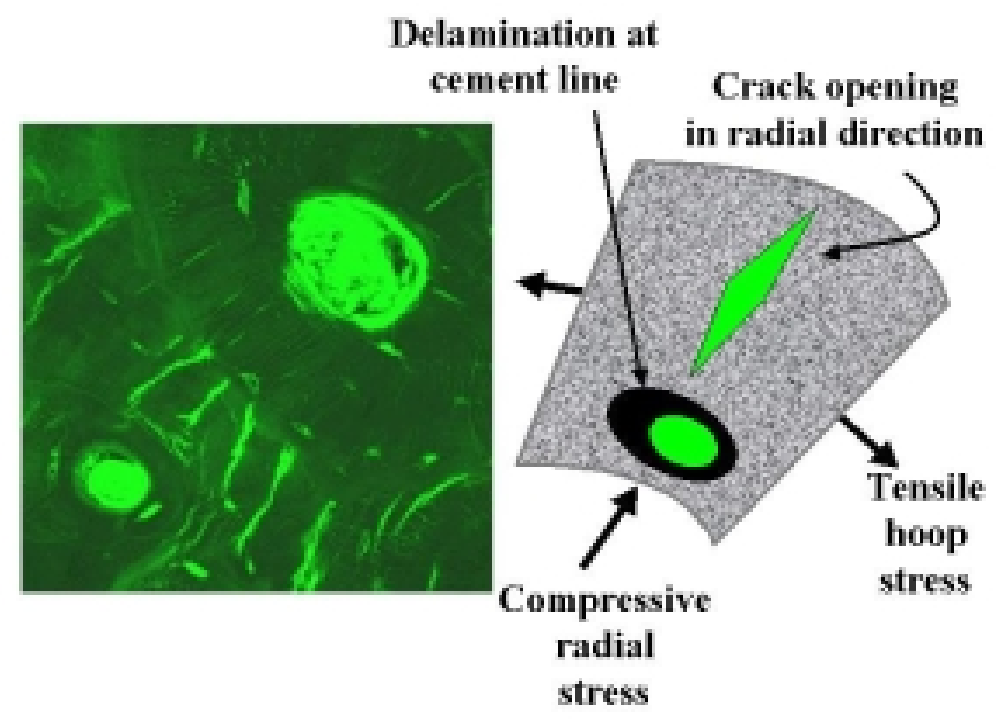

Figure 7.10 Tensile hoop and compressive radial stress tend to open cracks in the radial direction and cause delaminations between the cement lines and bone matrix. 
Damage occurred most frequently in the matrix ( $74 \%$ of the damage) which has also been shown to be less fracture resistant than osteonal tissue (Piekarski, 1970; Conrondan and Haworth, 1986; Yeni and Norman, 2000) and thus correlates well with the observation of only $13 \%$ of the damage occurring entirely within an osteon. The observation of the high frequency of matrix damage was also found in examinations of in vivo damage (Schaffler et al., 1995; Norman and Wang, 1997) and damage due to fatigue loading (Cater and Hayes, 1977; Schaffler et al., 1989; Schaffler et al., 1990).

Cracks that remain entirely within the cement line (types 4, 5, 6, 10 and 11) which represent $13 \%$ of the total damage is less than the number of cracks completely within the bone matrix, 33\%. Damage due to radial loading, therefore, appears to be more likely to occur in the matrix which has been shown to be less fracture resistant than osteonal tissue (Conrondan and Haworth, 1986; Piekarski, 1970).

Of the $74 \%$ of the damage observed in the matrix, $41 \%$ had at least one end of the crack at a cement line (types 2 and 9). It appears that the cement line influences crack behavior in this loading environment, a suggested role of the cement line based on in vivo observations (Burr et al., 1988; Schaffler et al., 1995; Norman and Wang, 1997) and on observations of bone after fatigue loading (Carter and Hayes, 1977; Schaffler et al., 1989; Schaffler et al., 1990).

Cracks along the cement line (type 3) occurred with low frequency and involve delaminations at the cement line-matrix interface. This loading environment, tensile hoop and compressive radial stresses acting perpendicular to the direction of the osteon axis, tends to delaminate osteons from interstitial bone along weak cement line interfaces (Burr et al., 1988).

The remaining group of "other" damage consists of microcracks that pass completely through the cement line (types $7,8,12,13$ and 14) which accounts for only $7 \%$ of all the damage observed compared to the $41 \%$ of damage with one end at a cement line. These data further suggest that the cement line influences crack behavior in this loading environment (Carter and Hayes, 1977; Burr et al., 1988; Schaffler et al., 1989; Schaffler et al., 1990; Schaffler et al., 1995; Norman and Wang, 1997). 
The observation that cracks frequently occur in the matrix and radial cracks are the most frequent, suggest that creep-failure of cortical bone initiates in the form of a radial matrix crack when the hoop stress component in the matrix just outside of the cement line of the osteon reaches a critical value. Whether matrix cracking or osteonal delaminations along the cement lines serve as a possible mechanism of creeping behavior at the ultrastructural level is still unclear.

Radial load in cortical bone tissue may result in microdamage. Damage in living bone tissue is repaired during a normal biological response involving osteoclasts that remove bone at the microcracks. The resorption of bone at these damage sites may increase porosity, reducing stiffness, and allow additional deformation to initially occur before modeling and remodeling take place (Frost, 1973). Understanding the relationship between intramedullary load and damage may prevent microcracking during stem insertion which could compromise implant stability.

Limitations of this study include the small number of specimens used. Additional specimens would improve the power of the statistical analysis. In summary, radial matrix microcracks and osteonal delaminations may serve as a possible damage mechanism of failure in this loading environment. Such damage may result in a loss of initial fixation compromising implant stability. This damage may also be a factor in determining the optimal degree of press-fit. 


\section{CHAPTER 8 \\ SUMMARY, CONTRIBUTIONS, RECOMMENDATIONS}

\subsection{Summary}

The results confirm the complexity of bone material behavior which is important to initial implant fixation. Fixation is dependent on the elastic response in bone to hold the implant until new bone grows. A solid press-fit results in improved load transfer and decreases implant micromotion. After the implant is inserted into the femur, a certain amount of force is needed between the implant and bone to maintain initial implant stability. Based on the results of this study, creep behavior in the hoop direction may occur at low levels of hoop stress. This suggests that deformation of the bone is likely after implantation and the initial press-fit may decrease. The hoop creep behavior may be the result of a combination of deformation modes. Elongation and slipping of tropocollagen molecules, rearrangement of cross-links and fibril deformation within lamellae are several mechanisms contributing to creep deformation. Results of this study also suggest that if the hoop stress in bone is sufficiently high, fracture may result, if the hoop stress is not, creep deformation may allow the implant to subside within the bone canal. Subsidence may lead to a reestablishment of initial fixation or cause further creep behavior to occur. When the bone-implant environment reaches an equilibrium and the implant remains stable, the bone may grow to the stem after several weeks and hold it in place to provide long term load transfer (Blaha, 1998). The deformation of cortical bone observed in this study, may result in a loss of initial implant fixation or may cause fracture of the bone.

\subsection{Contributions}

The results from this study provide fundamental knowledge regarding the creep behavior of bone under loading that closely simulates in-vivo conditions of "press-fit" intramedullary prostheses post-insertion. Creep strain, creep strain rate and permanent strain exhibited linear behavior with increasing hoop stress, until a particular stress level, or threshold, where it began to exhibit nonlinear behavior. Although the geometry of the cylinders was different from that of intact bone, these results suggest that a threshold may 
exist for intact bone. A better understanding of this threshold may lead to improvements in cementless implant design.

The long-term creep behavior in this study was used to derive creep models as a function of constant stress and time. Models were derived for the general case, considering the variability of human bone, a model for creep behavior within 6 hours, and a model to predict behavior for times longer than 6 hours. These creep models can be directly applied to finite element models to study the effects of hoop creep behavior in both cemented and uncemented implant models.

Damage mechanisms in bone due to creep were studied. Characterizing damage resulting from radial loading will increase our understanding of damage mechanisms associated with initial and time-dependent damage progression responsible for creep failure of press-fit total hip arthroplasty. Radial matrix microcracks and osteonal delaminations may serve as a possible damage mechanism of failure in this loading environment.

\subsection{Recommendations}

1. The creep models derived from this study should be directly applied to cementless and cemented implant computer models to determine effects of creep on implant stability. The results should be compared to computer models including distinct creep models for each quadrant.

2. The initial hoop stiffness should be used in bone models to determine effects of the orthotropic assumption of bone versus the transversely isotropic assumption.

3. Static axial compressive load may be applied by the text fixture to the cylindrical specimen while it is experiencing internal pressure to simulate the effects of standing.

4. Dynamic axial compressive load may be applied using the text fixture and a servohydraulic testing machine to simulate the effects of walking. 
5. The strain on the surface of intact bone specimens may be measured while cones of different cross section geometries are applied, and held, with a servohydraulic testing machine to study the effects of stem design on creep behavior.

6. Damage could be examined on the cross section of a bone cylinder at different points in time after being loaded with constant pressure to better understand damage progression due to creep.

7. Internal pressure may be applied to bone cylinders of different thickness to study the effects of specimen thickness.

8. More long-term creep specimens may be tested at stress levels below and within the range used in this study to gain a better understanding of the relationship between stress and time to failure.

9. The long term testing procedure could be applied to intact bone specimens to develop a time to failure relationship that is more clinically useful.

10. A solenoid valve which has a higher operating pressure would be useful along with the multi-phase loading protocol to examine the relationship between creep strain, creep strain rate, and permanent strain at higher levels of internal pressure. 


\section{REFERENCES}

Ashman, R.B., Cowin, S.C., Van Buskirk, W.C. and Rice, J.C., 1984, A continuous wave technique for the measurement of the elastic properties of cortical bone, $J$. Biomechanics, 17: 349-361.

ASTM A 370; A2.3, 1990, Determination of transverse yield strength, Hydraulic ringexpansion method, 1990 Annual Book of Standards, American Society for Testing and Materials, 261-262, 292.

Babbitt, A.M., 1994, Osteoporosis, Orthopedics, 17, No. 10: 935-941.

Beer, F. and Johnston, R., 1991, Mechanics of Materials, McGraw-Hill.

Bessman, E.S., Carter, D.R., McCarthy, J.C. and Harris, W.H., 1982, Accuracy enhancement of in-vivo bone strain measurements and analysis, Transactions of the ASME, 104: 226-229.

Blaha, J.D., 1998, Press-fit femoral components, The Adult Hip, ed. J.J. Callaghan, A.G. Rosenberg and H.E. Rubash, Lippincott-Raven, Philadelphia, 1085-1091.

Blaha, J.D., Grappiolo, G., Gruen, T.A., Spotorno, L.S., Mancinelli, C., Romagnoli, S. and Ivaldo, N., 1993, Five to eight year follow-up of the uncemented CLS press-fit stem, Annual meeting of AAOS, San Francisco, CA.

Blaha, J.D., Spotorno, L. and Romagnoli, S., 1991, CLS press-fit total hip arthroplasty, Techniques Orthop, 6: 80-86.

Blasius, K., Cotta, H., Schneider, U. and Thomsen, M., 1993, CLS multicenter study-8 year results, Z Orthop. Ihre Grenzgeb, 131: 547-552.

Bonfield, W. and Datta, P.K, 1974, Impact fracture of compact bone in a shock tube, $J$. Mater. Sci. 9: 1609-1614.

Bonfield, W. and Li, C.H., 1967, Anisotropy of non elastic flow in bone, J. Applied Physics, 38(6): 2450-2455.

Boresi, A.P., Schmidt, R.J. and Sidebottom, O.M., 1993, Advanced Mechanics of Materials. John Wiley and Sons, New York.

Bowman, S.M., Gibson, L.J., Hayes, W.C. and McMahon, T.A., 1997, A mathematical model for the creep behavior of bone, Transactions of the $43^{\text {rd }}$ Annual Meeting, Orthopedic Research Society, Feb. 9-13, p. 799. 
Bowman, S.M., Gibson, L.J., Hayes, W.C. and McMahon, T.A., 1999, Results from demineralized bone creep tests suggest that collagen is responsible for the creep behavior of bone, J. Biomechanical Engineering, 121: 253-258.

Bowman, S.M., Zeind, J., Gibson, L.J., Hayes, W.C. and McMahon, T.A., 1996, The tensile behavior of demineralized bovine cortical bone, J. Biomech., 29: 1497-1501.

Boyce, T.M., Fyhrie, D.P., Glotkowski, M.C., Radin, E.L. and Schaffler, M.B., 1998, Damage type and strain mode associations in human compact bone bending fatigue, J. Orthopedic Research, 16: 322-329.

Brainsky, A., Fox, K.M., Epstein, R., Glick, H., Hawkes, W., Lydick, E., Kashner, T.M., Zimmerman, S.I. and Magaziner, J., 1995, The economic cost of hip fractures in the elderly, Transactions of 41st Annual Meeting, Orthopedic Research Society, February 13-16, Orlando, FL, p. 456.

Brody, J.A., 1985, Prospects for an aging population, Nature, 315 (6019): 463.

Brown, C.U., Kish, V.L. and Norman, T.L., 1998, Circumferential strain around femoral cylinders due to simulated press-fit loading, Advances in Bioengineering, ASME BED-Vol. 39: 409-410.

Brown, C.U., Kish, V.L., Norman, T.L., Gruen, T.A. and Blaha, J.D., 2001, Circumferential creep of human cortical bone suggests threshold for press-fit stems, Transactions of the 47th Annual Meeting, Orthopaedic Research Society, San Francisco, CA, February 25-28.

Brown, C.U., Kish III, V.L., Vanscoy II, W.M., Norman, T.L. and Blaha, J.D., 2000a, Device for applying internal pressure to cylindrical specimens, Experimental Techniques, 24(4): 19-21.

Brown, C.U., Norman, T.L., Kish, V.L., Gruen, T.A. and Blaha, J.D., 1999, Transverse creep response in human femoral bone, $23^{\text {rd }}$ Annual Meeting, Am. Soc. Biomechanics, Oct. 21-21, Pittsburgh, PA., 164-165.

Brown, C.U., Parsamian, G.P., Norman, T.L., Kish, V.L., Gruen, T.A. and Blaha, J.D., $2000 \mathrm{~b}$, Mechanisms of creep induced failure in cylindrical cortical bone specimens due to radial loading. Advances in Bioengineering, ASME BED-Vol. 48: 223-224.

Bugbee, W.D., Culpepper, W.J., Engh, C. Jr. and Engh, C.A. Sr., 1997, Long-term clinical consequences of stress-shielding after total hip arthroplasty without cement, J. Bone Joint Surg., 79-A: 1007-1012.

Bulow, J.U., Scheller, G., Arnold, P., Synatschke, M. and Jani, L., 1996, Follow up (6-9 years) results of the uncemented CLS Spotorno stem, Arch. Orthop. Trauma Surg., 115(3-4): 190-194. 
Burr, D.B. and Stafford, T., 1990, Validity of the bulk-staining technique to separate artifactual from in vivo bone microdamage, Clin. Orthop. Rel. Res., 260: 305-308.

Burr, D.B. and Hooser, M., 1995, Alterations to the en bloc basic fuchsin staining protocol for the demonstration of microdamage produced in vivo, Bone, 17: 431-433.

Burr, D.B., Schaffler, M.B. and Frederickson, R.G., 1988, Composition of the cement line and its possible mechanical role as a local interface in human compact bone, $J$. Biomechanics, 21(11): 939-945.

Burstein, A.H., Reilly, D.T. and Martens, M., 1976, Aging of bone tissue: Mechanical properties, J. Bone Joint Surgery (Am), 58: 82-86.

Butler, C.A., Jones, L.C. and Hungerford, D.S., 1988, Initial implant stability of porous coated total hip femoral components: a mechanical study of micromovement. Trans. of the $34^{\text {th }}$ Annual Meeting, Orthopedic Research Society, Atlanta, GA, p. 549.

Byars, E.F., Snyder, R.D. and Plants, H.L., 1983, Engineering of Deformable Bodies, $4^{\text {th }}$ Edition, Harper and Row, New York.

Cabanela, M.E., 1990, Results of uncemented THA with the osteonics prosthesis, Hip Society, Scottsdale, AZ, Sept.

Caler, W.E. and Carter, D.R., 1989, Bone creep-fatigue damage accumulation, $J$. Biomechanics, 22: 625-635.

Carter, D.R. and Caler, W.E., 1983, Cycle-dependent and time-dependent bone fracture with repeated loading, J. Biomechanical Engineering, 105: 166-170.

Carter, D.R. and Caler, W.E., 1985, A cumulative damage model for bone fracture. $J$. Orthop. Res., 3: 84-90.

Carter, D.R. and Hayes, W.C., 1977, Compact bone fatigue damage - I. Residual strength and stiffness, J. Biomechanics, 10: 325-337.

Carter, D.R. and Hayes, W.C., 1977a, Compact bone fatigue damage. A microscopic examination, Clin. Orthop., 127: 265-274.

Chang, P.B., Robie, B.H., and Bartel, D.L., 1999, Preclinical cost analysis of orthopaedic implants: a custom versus standard cementless femoral component for revision total hip arthroplasty, J. Biomechanics, 32: 1309-1318.

Christensen, R.M., 1982, Theory of Viscoelasticity: An Introduction, $2^{\text {nd }}$ ed., Academic Press, New York. 
Clohisy, J.C. and Harris, W.H., 1999, The Harris-Galante uncemented femoral component in primary total hip replacement at 10 years, J. Arthrop., 14(8): 915-917.

Cohen, R.E., Hooley, C.J. and McCrum, N.G., 1976, Viscoelastic creep of collagenous tissue, J. Biomechanics, 9: 175-184.

Conrondan, G. and Haworth, W.L., 1986, A fractographic study of human long bone, $J$. Biomechanics, 19: 207-218.

Courtney, A.C., Wachtel, E.F., Myers, E.R. and Hayes, W.C., 1995, Age related reductions in the strength of the femur tested in a fall-loading configuration, J. Bone and Joint Surgery, 77-A: 387-395.

Cowan, P.M., North, A.C.T. and Randall, J.T., 1995, X-ray diffraction studies of collagen fibers, Symp. Soc. Exp. Biol., 9: 115-126.

Cowin, S.C., 1989, Bone Mechanics, CRC Press, Boca Raton, FL.

Cowin, S.C., Van Buskirk, W.C. and Ashman, R.B., in Handbook of Engineering, Skalak, R. and Chen, S., eds., McGraw-Hill, New York, 1987.

Cristescu, N. and Suliciu, I., 1982, Viscoplasticity. Martinus Nijhoff, The Hague.

Crowninshield, R.D., Pederson, D.R. and Brand, R.A., 1980, A measurement of proximal femur strain with total hip arthroplasty, Transactions of the ASME, 104: 230-233.

Crowninshield, R.D. and Pope, M.H., 1974, The response of compact bone in tension at various strain rates, Annals of Biomedical Engineering, 2: 217-225.

Crowninshield, R.D. and Tolbert, J.R., 1983, Cement strain measurement surrounding loose and well-fixed femoral component stems, J. Biomedical Materials Research, 17: 819-828.

Cummings, S.R., Black, D.M., and Rubin, S.M., 1989, Lifetime risks of hip, colles' or vertebral fracture and coronary heart disease among white postmenopausal women, Arch. Intern. Med., 149: 2445-2448.

Cummings, S.R., Kelsey, J.L, Nevitt, M.C., O’Dowd, K.J., 1985, Epidemiology of osteoporosis and osteoporotic fractures, Epidemiol. Rev., 7: 178-208.

Cummings, S.R., Rubin, S.M., and Black, D.M., 1990, The future of hip fractures in the United States: numbers, costs and potential effects of postmenopausal estrogen, Clin. Orthop., 252: 163-166.

Currey, J.D., 1962, Stress concentrations in bone, Q. J. Microsc. Sci., 103: 111-113. 
Currey, J.D., 1965, Anelasticity in bone and echinoderm skeletons, J. Exp. Biol., 43: 279292.

Currey, J.D., 1988, The effects of porosity and mineral content on the Young's modulus of elasticity of compact bone, J. Biomechanics, 21: 131-139.

Currey, J.D., 1988, Strain rate and mineral content in fracture models of bone, $J$. Orthopaedic Research, 6: 32-38.

Daum, W.J. and Uchida, T., 1992, Early comparison of femoral components in hip arthroplasty. A preliminary study, Orthop. Rev., 21(3): 327-334.

Davidson, P.F., 1989, The contribution of labile crosslinks to the tensile behavior of tendons, Connective Tissue Research, 18: 293-305.

Dieter, G.E., 1986, Mechanical Metallurgy, McGraw Hill, New York, pp. 438-443.

Dodge, B.M., Fitzrandolph, R. and Collins, D.N., 1991, Noncemented porous-coated anatomic total hip arthroplasty, Clin. Orthop., 269: 16-24.

Dorr, L.D., Absatz, M., Gruen, T.A., Saberi, M. and Doerzbacher, J.F., 1990, Anatomic porous replacement hip arthroplasty: First 100 consecutive cases, Sem. Arthroplasty, 1(1): 77-86.

Dorr, L.D., Lewonowski, K., Lucero, M. Harris, M. and Wan, Z., 1997, Failure mechanisms of anatomic porous replacement I cementless total hip replacement, Clin. Orthop., 334: 157-167.

Dorr, L.D. and Wan, Z., 1996, Comparative results of a distal modular sleeve, circumferential coating, and stiffness relief using the Anatomic Porous Replacement II, J. Arthroplasty, 11(4): 419-428.

Edwards, B.J. and Perry III, H.M, 1994, Age-related osteoporosis, Clin. Geriatr. Med., 10: $575-588$.

Ellis, S.C., Servant, C.T.J., Morrison, P.J.M. and Ross, A.C., 1997, Spotorno-Morscher cementless hip arthroplasty: the medium-term results in patients 60 years and under, J. Bone Joint Surg. Br., 79-B (suppl.1): 95.

Engh, C.A., 1983, Hip arthroplasty with a Moore prosthesis with porous coating: a fiveyear study, Clin. Orthop., 176: 52-66.

Engh, C.A. and Bobyn, J.D., 1988, The influence of stem size and extent of porous coating on femoral bone resorption after primary cementless hip arthroplasty, Clin. Orthop., 231: 7-28. 
Engh, C.A., Bobyn, J.D. and Glassman, A.H., 1987, Porous-coated hip replacement, $J$. Bone Joint Surg., 69-B: 45-55.

Engh, C.A. Sr. and Culpepper, W.J., 1997, Femoral fixation in primary total hip arthroplasty, Orthopedics, 20(9): 771-773.

Eyb, R., Kutschera, H.P., Schartelmueller, T., Toma, C. and Zweymuller, K., 1993, Midterm experience with the cementless Zweymuller system. Results of a minimum five-year follow up study, Acta. Orthop. Belg., 59 (Suppl. 1): 138-143.

Farmer, M.E., White, L.R., Brody, J.A. and Bailey, K.R., 1984, Race and sex differences in hip fracture incidence, American Journal of Public Health, 74: 1374-1380.

Findley, W.N., Lai, J.S. and Onaran, K., 1976, Creep and Relaxation of Nonlinear Viscoelastic Materials, North-Holland Publishing Company, New York, pp. 1-7.

Fitzgerald, R.H. Jr., Brindley, G.W. and Kavanagh, B.F., 1988, The uncemented total hip arthroplasty intraoperative femoral fractures, Clin. Orthop., 235, 61-66.

Fondrk, M., 1989, An experimental and analytical investigation into the nonlinear constitutive equation of cortical bone, Ph.D. dissertation, Department of Mechanical and Aerospace Engineering, Case Western Reserve University.

Fondrk, M., Bahniuk, E.H. and Davy, D.T., 1990, Transient creep behavior of cortical bone, Transactions of the $36^{\text {th }}$ Annual Meeting, Orthopedic Research Society, Feb. 58, New Orleans, LA, p. 49.

Fondrk, M., Bahniuk, E. and Davy, D., 1997, Crack density versus crack size predictions for cortical bone using a penny-shaped crack model, ASME BED, 35: 379-380.

Fondrk, M.T., Bahniuk, E.H. and Davy, D.T., 1999a, A damage model for nonlinear tensile behavior of cortical bone, J. Biomechanical Engineering, 121: 533-541.

Fondrk, M.,T., Bahniuk, E.H. and Davy, D.T., 1999b, Inelastic strain accumulation in cortical bone during rapid transient tensile loading, J. Biomechanical Engineering, 121: 616-621.

Fondrk, M., Bahniuk, E., Davy, D.T. and Michaels, C., 1988, Some viscoplastic characteristics of bovine and human cortical bone, J. Biomechanics, 21: 623-630.

Frasca, P., Harper, R.A. and Katz, J.L., 1977, Collagen fiber orientations in human secondary osteons, Acta. Anat., 98: 1-13.

Frost, H.M., 1986, Bone microdamage: Factors that impair its repair, In: Current Concepts of Bone Fragility, ed: H.K. Uhthoff, Springer-Verlag, Berlin, pp. 123-239. 
Frost, H.M., 1973, Bone modeling and skeletal modeling errors, Charles C Thomas Publisher, Springfield, IL, pp. 80.

Fung, Y.C., 1981, Biomechanics: Mechanical Properties of Living Tissues, SpringerVerlag, New York, NY, Chapter 7.

Fung, Y.C.B., 1968, Biomechanics: its scope, history and some problems of continuum mechanics in physiology, Appl. Mech. Rev., 21: 11-20.

Gallagher, J.C., Melton III, L.J., Riggs, B.L. and Bergstrath, E., 1980, Epidemiology of fractures of the proximal femur in Rochester, Minnesota, Clin Orthop, 150: 163-171.

Garrett and Grisham, 1999, Biochemistry, $2^{\text {nd }}$ edition, Saunders College Publishing.

Graves, E.J., 1994, 1992 Summary: National Hospital Discharge Survey, Advance Data, April 8, 249:1-12.

Gruen, T., 1997, A simple assessment of bone quality prior to hip arthroplasty: Cortical index revisited, Acta Orthopaedica Belgica, 63: 20-27.

Haddad, R.J., Cook, S.D. and Brinker, M.R., 1990a, A comparison of three varieties of noncemented porous-coated hip replacement, J. Bone Joint Surg. Br., 72(1): 2-8.

Haddad, R.J. Jr., Skalley, T.C., Cook, S.D., Brinker, M.R., Cheramie, J., Meyer, R. and Missry, J., 1990b, Clinical and roentgenographic evaluation of noncemented porouscoated anatomic medullary locking (AML) and porous-coated anatomic (PCA) total hip arthroplasties, Clin. Orthop., 258: 176-182.

Hellman, E.J., Capello, W.N. and Feinberg, J.R., Omnifit cementless total hip arthroplasty. A 10-year average follow-up, Clin. Orthop., 364: 164-174.

Holbrook, T.L., Grazier, K., Kelsey, J.L., et al., 1984, The frequency of occurrence, impact and cost of selected musculosketelal conditions in the United States, American Academy of Orthopedic Surgeons, Chicago.

Huiskes, R., Janssen, J.D. and Slooff, T.J., 1981, A detailed comparison of experimental and theoretical stress-analysis off a human femur, In: Mechanical Properties of Bone, ed. S.C. Cowin, ASME, New York, NY, pp. 211.

Huo, M.H., Martin, R.P., Zatorski, L.E. and Keggi, K.J., 1995, Total hip arthroplasty using the Zweymuller stem implanted without cement. A prospective study of consecutive patients with minimum 3-year follow-up period, J. Arthrop., 10(6): 793799.

Hustosky, K.T., Norman, T.L., Kish, V.L., Blaha, J.D. and Gruen, T.A., 1996, The effects of creep on cement hoop stresses and axial displacement of a cemented 
femoral hip prosthesis in vitro. Transactions of the $42^{\text {nd }}$ Annual Meeting, Orthopedic Research Society, Atlanta, GA, Feb. 22-26, p. 423.

Hwang, S.K. and Park J.S., 1995, Cementless total hip arthroplasty with AML, PCA and HGP prostheses, Int. Orthop., 19(2): 77-83.

Jansson, V. and Refior, H.J., 1992, Clinical results and radiologic findings after cementless implantation of PCA stems in total hip replacement, Arch. Orthop. Trauma Surg., 111(6): 305-308.

Jasty, M., Henshaw, R.M., O’Conner, D.O. and Harris, W.H., 1993, High assembly strains and femoral fractures produced during insertion of uncemented femoral components, J. Arthroplasty, 8: 479-487.

Jasty, M., O'Connor, D.O., Henshaw, R.M., Harrigan, T.P. and Harris, W.H., 1994, Fit of the uncemented femoral component and the use of cement influence the strain transfer to the femoral cortex, J. Orthopaedic Research, 12: 648-656.

Jepsen, K.A., Pattin, C.A., Bensusan, J.S. and Davy, D.T., 1995, Viscoelastic behavior and damage accumulation for bovine cortical bone in torsion, BED ASME, 29: 249250 .

Junqueira and Carneiro, 1992, Basic Histology, $8^{\text {th }}$ edition, Prentice-Hall, International Inc.

Jurist, J.M. and Foltz, A.S., 1977, Human ulnar bending stiffness, mineral content, geometry and strength, J. Biomechanics, 10: 455-459.

Kachanov, L.M., 1958, On the time to failure under creep conditions, Izv Akad Nauk SSSR, 8: 26-31.

Kachanov, L.M., 1961, Problems of Continuum Mechanics (Contributions in honor of seventieth birthday of N. I. Muskhelishvili, 16 Feb. 1961), ed. by J.R.M. Radok, Philadelphia, Society of Industrial and Applied Mathematics, pp. 202-218.

Kavanagh, B.F., 1992, Femoral factures associated with total hip arthroplasties, Ortho. Clin. N. America, 23(2): 249-257.

Kavanagh, B.F., Ilstrup, D.M., Fitzgerald, R.H., 1989, Uncemented PCA total hip arthroplasty: 2 year results, Orthop. Rev. 18 (Suppl): 39-45.

Kelley, S.S., 1994, Periprosthetic femoral fractures, J. Am. Aca. of Orthop. Surg., 2: 164172.

Kim, Y.H. and Kim, V.E., 1992, Results of the Harris-Galante cementless hip prosthesis, J. Bone Joint Surg. Br., 74(1): 83-87. 
Kim, Y.H., Kim, J.S. and Cho, S.H., 1999, Primary total hip arthroplasty with a cementless porous-coated anatomic total hip prosthesis: 10- to 12-year results of prospective and consecutive series, J. Arthroplasty, 14(5): 538-548.

Knets, I.V., 1978, Mechanics of biological tissues. A review, Polymer Mechanics (translation of Mekhanika Polimerov), 13: 434-440.

Knets, I.V. and Malmeisters, A., Deformability and strength of human compact bone tissue, Mechanics of Biological Solids: Proc. Euromech Colloquium 68, Brankov, G., ed., Bulgarian Academy of Sciences, Sofia, 1977, 133.

Knets, I.V. and Vilks, Yu. K., 1975, Creep of compact human bony tissue under tension. Polymer Mechanics, 11: 543-547.

Knight, J.L., Atwater, R.D. and Guo, J., 1998, Clinical results of the midstem porouscoated anatomic uncemented femoral stem in primary total hip arthroplasty: a five- to nine-year prospective study, J. Arthroplasty, 13(5): 535-545.

Kyle, R., 1999, Tips and pearls periprosthetic fractures, Tech. Orthop, 14(2): 89-94.

Lachiewicz, P.F., Anspach, W.E. 3d and DeMasi, R., 1992, A prospective study of 100 consecutive Harris-Galante porous total hip arthroplasties. 2-5-year results, $J$. Arthroplasty, 7(4): 519-526.

Lakes, R., 1993, Materials with structural hierarchy, Nature, 361: 511-515.

Lakes, R.S. and Katz, J.L., 1974, Interrelationships among the viscoelastic functions for anisotropic solids: application to calcified tissues and related systems, $J$. Biomechanics, 7: 259-270.

Lakes, R.S. and Katz, J.L., 1979a, Viscoelastic properties of wet cortical bone - II. Relaxation mechanisms, J. Biomechanics, 12: 679-687.

Lakes, R.S. and Katz, J.L., 1979b, Viscoelastic properties of wet cortical bone - III. A constitutive equation, J. Biomechanics, 12: 689-698.

Lakes, R.S., Katz, J.L. and Sternstein, S.S., 1979, Viscoelastic properties of wet cortical bone - I. Torsional and biaxial studies, J. Biomechanics, 12: 657-678.

Lakes, R. and Saha, S., 1979, Cement line motion in bone, Science, 204: 501-503.

Lakes, R. and Saha, S., 1980, Long-term torsional creep in compact bone, $J$. Biomechanical Engineering, 102: 179-180. 
Lanyon, L.E., Hampson, W.G.J., Goodship, A.E. and Shah, J.S., Bone deformation recorded in vivo from strain gages attached to the human tibial shaft, Acta Orthop. Scand., 46: 256, 1975.

Lehninger, A.L., Nelson, D.L. and Cox, M.M., 1993, Principles of Biochemistry, $2^{\text {nd }}$ edition, Worth Publishers, NY.

Lemaitre, J. and Chaboche, J.L., 1990, Mechanics of solid materials, Cambridge University Press, Cambridge, UK.

Lewinnek, G.E., Kelsey, J., White, A.A. and Kreiger, N.J., 1980, The significance and comparative analysis of the epidemiology of hip fractures, Clin. Orthop., 152: 35-43.

Lockett, F.J., 1972, Nonlinear Viscoelastic Solids, Academic Press, New York, pp. 1-9.

Lugassy, A.A. and Korostoff, E., 1969, Viscoelastic behavior of bovine femoral cortical bone and sperm whale dentin. Research in Dental and Medical Materials, Plenum Press, New York.

Magaziner, J., Simonsick, E.M., Kashner, T.M., Hebel, J.R. and Kenzora, J.E., 1989, Survival experience of aged hip fracture patients, Am. J. Public Health, 79: 274-278.

Manley, M.T. and Averill, R.G., 1989, Biocompatible load transfer between implant and bone, Osteonics Corp.

Martell, J.H., Galante, J.O., Pierson, R.H., Jacobs, J.J., Rosenberg, A.G. and Maley, M., 1992, Clinical experience with primary cementless total hip arthroplasty, Chir. Organi. Mov., 77(4): 383-396.

Martell, J.H., Pierson, R.H., Jacobs, J.J., Rosenberg, A.G., Maley, M. and Galante, J.O., 1993, Primary total hip reconstruction with a titanium fiber-coated prosthesis inserted without cement, J. Bone Joint Surg. Am., 75(4): 554-571.

Martin, R.B. and Burr, D.B., 1988, Structure, Function and Adaptation of Compact Bone, Raven Press, New York.

Martin, R.B., Pickett, J.C., and Zinaich, S., 1980, Studies of skeletal remodeling in aging men, Clinical Orthopaed. Relat. Res., 149:268-282.

Mathur, S.K., Mont, M.A. and McCutchen, J.W., 1996, Intraoperative custom press-fit and standard press-fit femoral components in total hip arthroplasty. A comparison of surgery, charges, and early complications, Am. J. Orthop., 25(7): 486-491.

Mauch, M., Currey, J.D. and Sedman, A.J., 1992, Creep fracture in bones with different stiffnesses, J. Biomechanics, 25: 11-16. 
McCalden, R.W., McGeough, J.A., Barker, M.B. and Court-Brown, C., 1993, Agerelated changes in the tensile properties of cortical bone, J. Bone Joint Surgery, 75: 1193-1204.

McElhaney, J.H., 1966, Dynamic response of bone and muscle tissue, J. Appl. Physiol., 21: $1231-1236$.

Melnis, A.E., Knets, I.V., 1981, Age-related changes in the tensile creep properties of human compact bone tissue, Mechanics of Composite Materials, 17: 495-501.

Melnis, A.E. and Knets, I.V., 1982, Effect of the rate of deformation on the mechanical properties of compact bone tissue, Mechanics of Composite Materials, 18: 358-363.

Melnis, A.E., Knets, I.V. and Moorlat, P.A., 1979, Deformation behavior of human compact bone tissue upon creep under tensile testing, Mechanics of Composite Materials, 15: 574-579.

Melnis, A.E., Kregers, A.F., and Villerush, K.K., 1982, An evaluation of some factors affecting the creep properties of human compact bone tissue, Mechanics of Composite Materials, 17: 711-715.

Melnis, A.E. and Laizan, Ya. B., 1978, Nonlinear creep of human compact bone tissue upon stretching, Polymer Mechanics, 14: 82-84.

Melnis, A.E., Ozola, B.O. and Moorlat, P.A., 1981, Comparative characteristics of the creep properties of human compact bone tissue under various specimen storage and testing conditions, Mechanics of Composite Materials, 17: 355-360.

Melton III, L.J., 1988, Epidemiology of fractures. In: Osteoporosis: Etiology, Diagnosis and Management, eds. B.L. Riggs and L.J. Melton III. Raven Press, NY, pp. 133-154.

Miller, J., Krause, W.R., Krug, W.H. and Kalaby, L.C., 1982, Implant fixation. In Revision Total Hip Arthroplasty, eds. R.H. Turner and A.D. Scheller, Grune and Stratton, Inc., New York, p. 195.

Morscher, E.W., 1983, Cementless total hip arthroplasty, Clin. Orthop., 181: 76-91.

Mosler, E., Folkhard, W., Knorzer, E., Nemetschek-Gansler, H. and Nemetschek, T., Stress-induced molecular rearrangement in tendon collagen, J. Molecular Biology, 182: 589-596.

Murphy, S.B., Walker, P.S. and Schiller, A.L., 1984, Adaptive changes in the femur after implantation of an Austin Moore prosthesis, J. Bone Joint Surgery, 66-A: 437-443.

Nemetschek, Th., Riedl, H. and Jonak, R., 1979, Topochemistry of the binding of phosphotungstic acid to collagen, J. Mol. Biol., 133: 67-83. 
de Nies, F. and Fidler, M.W., 1996, The Harris-Galante cementless femoral component: poor results in 57 hips followed for 3 years, Acta. Orthop. Scand., 67(2): 122-124.

NIH, 1984, "Osteoporosis", National Institute of Health Consensus Development Conference Statement, April 2-4, 5, No. 3: 1-6.

Nicolella, D.P., Nicholls, A.E. and Lankford, J., 1998, Micromechanics of creep in cortical bone, Transactions of the $44^{\text {nd }}$ Annual Meeting, Orthopedic Research Society, New Orleans, LA, March 16-19, p. 137.

Norman, T.L. and Wang, Z., 1997, Microdamage of human cortical bone: incidence and morphology in long bones, Bone, 20: 375-379.

Oh, I. and Harris, W.H., 1978, Proximal strain distribution in the loaded femur, J. Bone Joint Surgery, 60-A: 75-82.

Onsten, I., Carlsson, A.S., Sanzen, L. and Besjakov, J., 1996, Migration and wear of a hydroxyapatite-coated prosthesis. A controlled roentgen stereophotogrammetric study, J. Bone Joint Surg. Br., 78(1): 85-91.

Otani, T., Whiteside, L.A. and White, S.E., 1993, Strain distribution in the proximal femur with flexible composite and metallic femoral components under axial and torsional loads, J. Biomedical Materials Research, 27: 575-585.

Parfitt, A.M., Drezner, M.K., Glorieux, F.H., Kanis, J.A., Malluche, H., Meunier, P.J., Ott, S.M. and Recker, R.R., 1987, Bone histomorphometry: standardization of nomenclature, symbols and units. J. Bone Mineral Res., 2: 595-610.

Park, J.B., 1979, Biomaterials: An Introduction. Plenum, New York, p. 172.

Park, H.C. and Lakes, R.S., 1986, Cosserat micromechanics of human bone: strain redistribution by a hydration sensitive constituent, J. Biomechanics, 19: 385-397.

Pattin, C.A., Jepsen, K.J., Bensusan, J.S. and Davy, D.T., 1995, Creep behavior of compact bone in tensile and compressive loading modes, BED ASME, 29: 247-248.

Penenberg, B.L., 1998, Management of periprosthetic fractures. In The Adult Hip, ed. J.J. Callaghan, A.G. Rosenberg, and H.E. Rubash, Lippincott-Raven, Philadelphia, pp. 1241-1267.

Perry III, H. and Morley, J., 1992, Hip fracture, In: Falls, balance and gait disorders in the elderly, ed. B. Vellas, M. Toupet, L. Rubenstein, et al., Paris, Elseiver. 
Petersilge, W.J., D’Lima, D.D., Walker, R.H. and Colwell, C.W. Jr., 1997, Prospective study of 100 consecutive Harris-Galante porous total hip arthroplasties. 4- to 8-year follow-up study, J. Arthroplasty, 12(2): 185-193.

Petty, W., 1991, Total hip arthroplasty: complications. In Total Joint Replacement, ed. W. Petty and W.B. Saunders, pp. 287-314.

Piekarski, K., 1970, Fracture of bone, J. Appl. Phys., 41: 215-233.

Poser, R.D., 1992, The bone-implant interface: the role of hydroxyapatite in optimizing long term fixation, Bio-Interfaces, Inc., Osteonics.

Poss, R., Walker, P., Robertson, D.D., Reiley, D.T., Ewald, F.C., Thomas, W.H. and Sledge, C.B., 1988b, Anatomic stem design for press-fit and cemented application in cementless total hip arthroplasty. In Fitzgerald, R.H., Jr. (ed.): Noncemented total hip arthroplasty. Phoenix, Raven Press.

Poss, R., Walker, P., Spector, M., Reilly, D.T., Robertson, D.D. and Sledge, C.B., 1988a, Strategies for improving fixation of femoral components in total hip arthroplasty, Clin. Orthop., 235: 181-194.

Purslow, P.P., Wess, T.J. and Hukins, D.W.L., 1998, Collagen orientation and molecular spacing during creep and stress-relaxation in soft connective tissues, J. Experimental Biology, 201: 135-142.

Rauber, A.A., 1876, Elasticitat und Festigkeit der Knochen, Anatomisch-physiologische Studie. Engelmann, Leipzig.

Reed, P.E. and Squires, H.V., 1974, Application of fracture mechanics to plastics deformed at high strain-rates, J. Mater. Sci., 9: 129-135.

Reid, R.H., DiGioia, A.M., Jaramaz, B. and Ghattas, O., 1995, Press fit implants yield high assembly strains in cortical bone, BED ASME, 29: 561-562.

Reilly, D.T. and Burstein, A.H., 1975, The elastic and ultimate properties of compact bone tissue, J. Biomechanics, 8: 393-405.

Rho, J.Y., Hobatho, M.C. and Ashman, R.B., 1995, Anatomical variation of mechanical properties of human cortical bone, BED ASME, 29: 239-240.

Rhoads, J.L., 1994, Basic explanations of creep processes: www.nuc.berkeley.edu/thyd/ne161/jlrhoads/creep.html, December.

Riggs, B.L. and Melton III, L.J.,1986, Involutional osteoporosis. N. Engl. J. Med., 314: 1676-1686. 
Rimnac, C.M., Petko, A.A., Santner, T.J. and Wright, T.M., 1993, The effect of temperature, stress and microstructure on the creep of compact bovine bone, $J$. Biomechanics, 26: 219-228.

Rimnac, C.M., Petko, A.A., Santner, T.J. and Wright, T.M, 1993a, Creep of compact human bone, Trans. ORS, 18: 172.

Rimnac, C.M., Petko, A.A. and Wright, T.M., 1991, Creep of compact bone: effects of temperature and stress on bovine lamellar microstructure, Trans. ORS, 18: 152.

Ring, P.A., 1978, Five to fourteen year interim results of uncemented total hip arthroplasty, Clin. Orthop., 137: 87.

Robertson, D.D., Walker, P.S., Hirano, S.K., Zhou, X.M., Granhholm, J.W. and Poss, R., 1988, Improving the fit of press-fit hip stems, Clin. Orthop., 228: 134-140.

Robinson, R.P. and Clark, J.E., 1996, Uncemented press-fit total hip arthroplasty using the Identifit custom-molding technique. A prospective minimum 2-year follow-up study, J. Arthroplasty, 11(3): 247-254.

Robinson, R.P., Deysine, G.R. and Green, T.M., 1996, Uncemented total hip arthroplasty using the CLS stem: a titanium alloy implant with a corundum blast finish. Results at a mean 6 years in a prospective study, J. Arthroplasty, 11(3): 286-292.

Rohlmann, A., Mossner, U., Bergmann, G. and Kolbel, R., 1982, Finite-element analysis and experimental investigation of stresses in a femur, J. Biomedical. Engineering, 4: 241-246.

Saito, S, Ryu, J., Oikawa, H. and Honda, T., 1997, Clinical results of Harris-Galante total hip arthroplasty without cement. Follow-up study of over five years, Bull. Hosp. Jt. Dis., 56(4): 191-196.

Sakkers, R.J.B., Valkema, R., de Wijn, J.R., Lentjes, E.G.W.M., van Blitterswijk, C.A. and Rozing, P.M., 1995, The intramedullary hydraulic pressure tolerance of the human femur, Clin. Orthop. Rel. Res., 311: 183-189.

Sasaki, N. and Odajima, S., 1996a, Stress strain curve and Young's modulus of collagen molecule as determined by X-ray diffraction technique, J. Biomech., 29: 655-658.

Sasaki, N. and Odajima, S., 1996b, Elongation mechanism of collagen fibrils and forcestrain relations of tendon at each level of structural hierarchy, J. Biomech., 29: 11311136.

Sasaki, N., Shukunami, N., Matsushima, N. and Izumi, Y., 1999, Time-resolved X-ray diffraction from tendon collagen during creep using synchrotron radiation, $J$. Biomechanics, 32: 285-292, 1999 
Schaffler, M.B., Burr, D.B. and Radin, E.L., 1989, Mechanical and morphological effects of strain rate on fatigue in compact bone, Bone, 10: 207-214.

Schaffler, M.B., Choi, K. and Milgrom, C., 1995, Aging and matrix microdamage accumulation in human compact bone, Bone, 17: 521-525.

Schaffler, M.B., Radin, E.L. and Burr, D.B.,1990, Long-term fatigue behavior of compact bone at low strain magnitude and rate, Bone, 11: 321-326.

Scheller, A.D. Jr., and D'Errico, J., 1982, Hip biomechanics and prosthetic design and selection in revision total hip replacement. In Revision Total Hip Arthroplasty, eds. R.H. Turner and A.D. Scheller, Grune and Stratton, Inc., New York, p. 66.

Schneider, E., Eulenberger, J., Steiner, W., Eskilsson, G., and Perren, S. M., 1987, Trans.

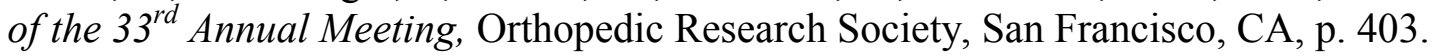

Schwartz, J.T., Mayer, J.G. and Engh, C.A., 1989, Femoral fracture during non-cemented total hip arthroplasty, J. Bone Jt. Surg., 71: 1135-1142.

Scott, R.D., Turner, R.H., Leitzes, S.M. and Aufranc, O.E., 1975, Femoral fractures in conjunction with total hip replacement, J. Bone Joint Surg., 57: 494-501.

Sedlin, E.D., 1965, A rheologic model for cortical bone, A study of the physical properties of human femoral samples, Acta Orthopaedica Scandinavica, S83: 1-77.

Shigley, J.E. and Mischke, C.R., 1989, Mechanical Engineering Design, McGraw-Hill, New York, pp. 58-60.

Simkin, A. and Robin, G., 1974, Fracture formation in differing collagen fiber pattern of cortical bone, J. Biomechanics, 7: 183-188.

Skrzypek, J.J. and Hetnarski, R.B., 1993, Plasticity and Creep, CRC Press, Inc., Boca Raton, FL.

Smith, E. and Harris, W.H., 1995, Increasing prevalence of femoral lysis in cementless total hip arthroplasty, J. Arthroplasty, 10(4): 407-412.

Smith, R. and Keiper, D., 1965, Dynamic measurement of viscoelastic properties of bone, Am. J. Med. Elec., 4: 156-160.

Smith, J.W. and Walmsley, R., 1959, Factors affecting the elasticity of bone, J. Anat., 93; 503-523. 
Spotorno, L., Romagnoli, S., Ivaldo, N., Grappiolo, G., Bibbiani, E., Blaha, D.J. and Gruen, T.A., 1993, The CLS system. Theoretical concept and results, Acta. Orthop. Belg., 59 (Suppl. 1): 144-148.

Spotorno, L., Schenk, R.K., Dietschi, C., Romagnoli, S. and Mumenthaler, A., 1987, Personal experiences with uncemented prostheses, Orthopade, 16(3): 225-238.

Stryer, L., 1995, Biochemistry, $4^{\text {th }}$ edition, W.H. Freeman and company, NY.

Sugiyama, H., Whiteside, L.A. and Engh, C.A., 1992, Torsional fixation of the femoral component in total hip arthroplasty. The effect of surgical press-fit technique, Clin. Orthop., 275: 187-193.

Sugiyama, H., Whiteside, L.A., Engh, C.A. and Otani, T., 1994, Late mechanical stability of the proximal coated AML prosthesis, Orthopedics, 17(7): 583-588.

Suzuki, E., Fraser, R.D.B. and MacRae, T.P., 1980, Role of hydroxyproline in the stabilization of the collagen molecule via water molecules. Int. J. Biol. Macromol., 2: 54-56.

Tanabe, Y. and Kobayashi, K., 1994, Anisotropy in the dynamic non-linear viscoelastic properties of bovine compact bone, J. Mat. Sci.: Materials in Medicine, 5: 397-401.

Timoshenko, S.P. and Goodier, J.N., 1970, Theory of Elasticity, McGraw-Hill, New York.

Tong, K.M., Hsu, K.C., Ku, M.C. and Lee, T.S., 1993, Two to five year follow-up of Harris-Galante cementless total hip arthroplasty, Chung Hua I Hsueh Tsa Chih (Taipei), 52(3): 137-144.

Turner, R.H. and Emerson, R.H. Jr., 1982, Femoral revision total hip arthroplasty. In Revision Total Hip Arthroplasty, eds. R. H. Turner and A. D. Scheller, Grune and Stratton, Inc., New York, p. 97.

Utenkin, A.A. and Sveschnikova, A.A., The effect of prolonged loading on the deformation properties of compact bone matter, Arkh. Anat. Gistol. Embriol., 64: 14$20,1973$.

Van Buskirk, W.C., 1989, Elementary stress analysis of the femur and tibia, In: Bone Mechanics, ed: S.C. Cowin, CRC Press, Boca Raton, FL, pp. 43-51.

Van Buskirk, W.C. and Ashman, R.B., 1981, The elastic moduli of bone, In: Mechanical Properties of Bone, ed. S.C. Cowin, ASME, New York, NY, pp. 131- 143. 
Vanscoy, W.M., 1996, Circumferential behavior of human cortical bone loaded by internal pressure, MSME Thesis, West Virginia University, Morgantown, West Virginia.

Vanscoy, W.M., Kish, V., Gruen, T. and Norman, T.L., 1997, Transverse strength of human femoral bone loaded by internal pressure, BED ASME, 35: 251-252.

Vashishth, D., Koontz, J., Qiu, S., Cannon-Lundin, D., Schaffler, M.B. and Fyhrie, D.P., 1999, Characterization of diffuse damage in human trabecular bone, Transactions of the $45^{\text {th }}$ Annual Meeting, Orthopedic Research Society, p. 765.

Verdonschot, N. and Huiskes, R., 1998, Surface roughness of debonded straight-tapered stems in cemented THA reduces subsidence but not cement damage, Biomaterials, 19: $1773-1779$.

Wang, Z., 1995, Microstructure and microdamage of human cortical bone, MSME thesis, West Virginia University, Morgantown, WV, July.

Wertheim, G., 1847, Memoire sur l'elasticite et la cohesion des principaux tissus du corps humain. Annals Chim. Phys., 21:385-414.

Whiteside, L.A., White, S.E., Engh, C.A. and Head, W., 1993, Mechanical evaluation of cadaver retrieval specimens of cementless bone-ingrown total hip arthroplasty femoral components, J. Arthroplasty, 8(2): 147-155.

Woolson, S.T. and Maloney, W.J., Cementless total hip arthroplasty using a porouscoated prosthesis for bone ingrowth fixation. $3 \frac{1}{2}$-year follow-up, J. Arthroplasty, 7 (Suppl): 381-388.

Wright, T.M. and Hayes, W.C., 1976, Tensile testing of bone over a wide range of strain rates: Effects of strain rate, microstructure and density, Med. Biomech. Eng., 14: 641679.

Yamada, H.,1970, Strength of Biological Materials, Williams and Wilkins, Baltimore.

Yeni, Y.N. and Norman, T.L., 2000, Calculation of porosity and osteonal cement line effects on the effective fracture toughness of cortical bone in longitudinal crack growth, J. Biomed. Mater. Res., 51(3) 504-509.

Yoon, H.S. and Katz, J.L., 1976, Ultrasonic wave propagation in human cortical bone. II. Measurements of elastic properties and micro-hardness, J. Biomechanics, 9: 459.

Zhou, X.M., Walker, P.S. and Robertson, D.D., 1990, Effect of press-fit femoral stems on strains in the femur, J. Arthroplasty, 5: 71-82. 
Zioupos, P., Currey, J.D. and Hamer, A.J., 1999, The role of collagen in the declining mechanical properties of aging human cortical bone, J. Biomedical Materials Research, 45: 108-116.

Zweymuller, K., 1986, A cementless titanium hip endoprosthesis system based on pressfit fixation: basic research and clinical results. In Anderson, L.D. (ed.): AAOS Instructional Course Lectures, vol. 35, St. Louis. C.V. Mosby, pp. 203-225. 


\section{CURRICULUM VITAE}

\section{CHRISTOPHER U. BROWN}

Email: cb0626@yahoo.com

\section{EDUCATION}

West Virginia University, Morgantown, WV

Ph.D. Mechanical Engineering (Certificate in Bioengineering) - May 2001

Dissertation: Time-Dependent Circumferential Deformation of Cortical Bone Subjected to Internal Radial Loading

Committee Members: Dr. T.L. Norman, Dr. J.D. Blaha, Mr. T. Gruen, Dr. E. Barbero, Dr. V. Mucino

M.S. Mechanical Engineering (Certificate in Bioengineering) - December 1995

Thesis: Fracture Toughness of Human Cortical Bone from the Proximal Femur

Advisor: Dr. T.L. Norman

B.S. Aerospace Engineering - May 1994

Engineer in Training, State of West Virginia Certificate \# EI 6507

\section{RESEARCH EXPERIENCE}

Graduate Research Assistant, Department of Mechanical and Aerospace Engineering and Orthopedics, Musculoskeletal Research Center, West Virginia University, PO Box 9196, Morgantown, WV 26506-9196

- Colonizing, splitting and maintaining stromal cells derived from rabbit bone marrow, December 2000 - present

- Researching topics involving spinal fusion techniques with osteoinductive materials such as bone morphogenetic proteins (BMP), transforming growth factor- $\beta$ (TGF- $\beta$ ), insulin-like growth factors (IGF) and demineralized bone matrix (DBM) and biocompatible osteoconductive carriers such as hydroxyapatite-tricalcium phosphate (HA-TCP), ceramics and collagen sponges, February 2000 - present

- Conducted internal pressurization tests of human bone cylinders for the purpose of determining the time-dependent circumferential response of human bone to an internal radial load, determined damage mechanisms and conducted morphological analysis of specimens. The mechanical tests model the behavior of bone in response to a press-fit style total hip replacement in partial fulfillment of the Ph.D. degree, January 1996 - present

- Conducted observations using Optimus Image Analysis software to measure morphological parameters from slides of rat femurs after having been subjected to different levels of gravity and exercise (NASA Space Consortium), Summer 1998

- Designed and conducted mechanical tests to compare bond strength between orthodontic resin adhesives and resin-reinforced glass ionomer orthodontic cement, Fall 1997 
- Determining tension and shear fracture toughness and morphology of the proximal human femur in partial fulfillment of the degree of Master of Science. This project continues to expand and complete the donor database, May 1994 - present

Student Researcher, Department of Mechanical and Aerospace Engineering, West Virginia University

- A semester research project was conducted to analyze the fracture surface of human cortical bone fracture toughness specimens using the scanning electron microscope (SEM), Fall 1998

- During the senior year of undergraduate study, a semester of research was conducted involving the interface strength between surgical bone cement and stainless steel, the results of this preliminary study were used for subsequent research projects, Spring 1994

- The senior undergraduate design project concluded its findings in a paper presented at the NASA conference. A defense system was proposed to help eliminate orbital debris in Earth's atmosphere, Spring 1994

\section{TECHNICAL SKILLS}

Analysis Techniques:

- Light, Laser Scanning Confocal and Scanning Electron Microscopy

- Radiographic assessment of human bone

- Mechanical testing using a servohydraulic testing machine

- Histomorphometrical analysis of specimens with basic fuchsin and hematoxylin-eosin staining techniques using image analysis software

- Applying strain gages to metal and bone

- Experience with statistical analysis software (JMP) for analyzing data and creating databases

- Histological preparation for biochemical analysis

- Microdamage assessment of bone

\section{Computer:}

- Experienced with both IBM and Macintosh based operating systems and software and familiar with Unix environment

- Experienced with the following software packages: Microsoft Word, Excel, PowerPoint, JMP, KaleidaGraph, Labtech Notebook, Optimus Image Analysis Software, MacDraft, Netscape Navigator, NIH Image 1.57, Autocad, Coplot, Matlab, Quatro Pro, ABAQUS

- Experienced with CNC programming, QBasic, and FORTRAN

\section{General:}

- Familiar with techniques involved in colonizing, splitting and maintaining stromal cells

- Excellent verbal and written communication skills

- Strong organizational and project management skills

- Skilled as a test engineer in a laboratory setting

- Working knowledge of laboratory environment including safety, Bloodborne Pathogen, and Chemical Hygiene training 


\section{TECHNICAL SOCIETIES}

Society for the Advancement of Materials and Process Engineering (SAMPE), West Virginia University Student Chapter, 1998-present

Biomedical Engineering Society (BMES), West Virginia University Student Chapter founding member, Vice President, 1994 - 1998

American Society of Mechanical Engineers (ASME), 1995-present

\section{HONORS}

Invited to attend the $22^{\text {nd }}$ SAMPE Europe International Conference as the North American student speaker to present research, CNIT Conference Centre, La Defense, Paris, France, March 26-29, 2001.

Co-Chair, Bone Mechanics II Session, Bioengineering Division, 2000 ASME International Mechanical Engineering Congress and Exposition, Winter Annual Meeting, November 10, Orlando, Florida.

Graduate Student Research Award, Musculoskeletal Research Center, West Virginia University, June 2, 2000. This award was presented in recognition for outstanding accomplishments in musculoskeletal research.

First place, SAMPE Student Paper Competition Finalist, Doctorate Level, 2000 SAMPE Student Symposium during the 45th International Symposium and Exhibition, Long Beach, CA, May 2125, 2000. Presentation titled: "Time-dependent Circumferential Deformation of Bone Specimens Subjected to Internal Radial Loading". Four finalists were chosen from the papers submitted from North America to present their work where the final judging took place.

First place, Baltimore-Washington, D.C. SAMPE Chapter 2000 Student Symposium, for presentation of poster titled: "Circumferential Strain Around Femoral Cylinders Due To Simulated Press-Fit Loading". The West Virginia University student chapter attended the symposium held at the University of Maryland, College Park, on February 9, 2000.

Graduate Student Research Award, Musculoskeletal Research Center, West Virginia University, May 21, 1999. This award was presented in recognition for outstanding accomplishments in musculoskeletal research.

SAMPE Student Paper Competition Finalist, Doctorate Level, 1998 SAMPE Student Symposium during the 43rd International Symposium and Exhibition, Anaheim, CA, May 31 to June 4, 1998. Paper and presentation titled: "The Microstructure of Human Bone Influences Fracture Toughness Similar to Fiber-Reinforced Composites". Eight finalists were chosen from the papers submitted from around the world to present their work where the final judging took place.

First place, Baltimore-Washington, D.C. SAMPE Chapter 1998 Student Symposium, for presentation of poster titled: "The Influence of Bone Microstructure and Composition on the Fracture Toughness of Human Cortical Bone". The West Virginia University student chapter attended the symposium held at the University of Maryland, College Park, on February 11, 1998. 
Third place finalist, ASME Student Paper Competition in the Bioengineering Division, Master's Level, 1995 ASME Winter Annual Meeting, San Francisco, CA, November, 1995. Paper and presentation titled: "Fracture Toughness of Human Cortical Bone from the Proximal Femur". Six finalists were chosen from the entries submitted from across the nation to present their work where the final judging took place.

West Virginia University College of Engineering Dean's Honor List, fall semester 1993, fall/spring 1994.

Athletic Director's Academic Honor Roll, fall and spring semesters 1993.

Freshman Scholarship award for fall semester 1990.

\section{ACTIVITIES}

- Substitute instructor for undergraduate and graduate classes (Statics, Mechanics of Materials and Advanced Mechanics of Materials), 1999-2001.

- Participated in mock trial, McLain versus Barber, as expert witness Dr. Michael Gratch, October 20, 1998, West Virginia University Law School. The case is a personal injury action arising from an automobile accident.

- Participated in orientation programs to promote awareness of the Biomedical Engineering Society at West Virginia University. Discussed bioengineering research at WVU and application of engineering tools and materials in the medical field with undergraduate engineering students, Spring 1996-1998.

- West Virginia University, Cross Country, Indoor Track, Outdoor Track, Fall 1990 - Spring 1993

\section{PUBLICATIONS}

\section{Journal Articles}

Brown, C.U., Kish III, V.L., Vanscoy II, W.M., Norman, T.L. and Blaha, J.D., 2000, Device for applying internal pressure to cylindrical specimens. Experimental Techniques, 24(4): 19-21.

Brown, C.U., Yeni, Y.N. and Norman, T.L., 2000, Fracture toughness is dependent on bone location-a study of the femoral neck, femoral shaft, and the tibial shaft. Journal of Biomedical Materials Research, 49(3): 380-389.

Kocamis, H., Yeni, Y.N., Brown, C.U., Kenney, P.B., Kirkpatrick-Keller, D.C. and Killefer, J., 2000, Effect of in ovo administration of insulin-like growth factor-I on composition and mechanical properties of chicken bone. Poultry Science, 79(9): 1345-1350.

Shammaa, I., Ngan, P., Kim, H., Kao, E., Gladwin, M., Gunel, E. and Brown, C., 1999, Comparison of bracket debonding force between two conventional resin adhesives and a resinreinforced glass ionomer cement: an in vitro and in vivo study. The Angle Orthodontist, 69(5): 463-469.

Norman, T.L, Yeni, Y.N., Brown C.U. and Wang, Z., 1998, Influence of microdamage on fracture toughness of the human femur and tibia. Bone, 23, No. 2: 303-306. 
Yeni, Y.N., Brown, C.U. and Norman T.L., 1998, Influence of bone composition and apparent density on fracture toughness of the human femur and tibia. Bone, 22, No. 1: 79-84.

Yeni, Y.N., Brown, C.U., Wang, Z. and Norman, T.L., 1997, The influence of bone morphology on fracture toughness of the human femur and tibia. Bone, 21, No. 5: 453-459.

\section{Conference Papers}

Brown, C.U., Kish, V.L., Norman, T.L., Gruen, T.A. and Blaha, J.D., 2001, Circumferential creep of human cortical bone suggests threshold for press-fit stems. Transactions of the 47th Annual Meeting, Orthopaedic Research Society, San Francisco, CA, February 25-28.

Brown, C.U., Parsamian, G.P., Norman, T.L., Kish, V.L., Gruen, T.A. and Blaha, J.D., 2000, Mechanisms of creep induced failure in cylindrical cortical bone specimens due to radial loading. Advances in Bioengineering, ASME BED-Vol. 48: 223-224.

Brown, C.U., Kish, V.L., Parsamian, G.P., Norman, T.L., Gruen, T.A. and Blaha, J.D., 2000, Time-dependent circumferential deformation of bone specimens subjected to internal radial loading. Student paper for the $45^{\text {th }}$ International SAMPE Symposium, Long Beach, CA, May 21 25.

Brown, C.U., Norman, T.L., Kish, V.L., Gruen, T.A. and Blaha, J.D., 1999, Transverse creep response in human femoral bone. Transactions of the $23^{\text {rd }}$ Annual Meeting, American Society of Biomechanics, October 21-23, Pittsburgh, PA, pp. 164-165.

Brown, C.U., Kish, V.L. and Norman, T.L., 1998, Circumferential strain around femoral cylinders due to simulated press-fit loading. Advances in Bioengineering, ASME BED-Vol. 39: 409-410.

Brown, C.U., Yeni, Y.N. and Norman, T.L., 1998, Fracture toughness of the femoral neck, femoral shaft, and tibial shaft in aged bone. Advances in Bioengineering, ASME BED-Vol. 39: 279-280.

Yeni, Y.N., Brown, C.U. and Norman T.L., 1998, Fracture toughness of cortical bone from the femur correlates with radiogrammetrical parameters in the elderly. Advances in Bioengineering, ASME BED-Vol. 39: 273-274.

Brown, C.U., Yeni, Y.N. and Norman, T.L., 1998, The microstructure of human bone influences fracture toughness similar to fiber-reinforced composites. Student paper for the $43^{\text {rd }}$ International SAMPE Symposium, Anaheim, CA, May 31 to June 4.

Shammaa, I., Ngan, P., Kim, H., Kao, E., Gladwin, M. and Brown, C., 1998, Comparison of bone strength between resin and reinforced glass ionomer orthodontic adhesive. American Association of Orthodontists Annual Meeting, May 17.

Yeni, Y.N., Brown, C.U. and Norman, T.L., 1997, The influence of bone microstructure and composition on the fracture toughness of human cortical bone. Proceedings of the 1997 Bioengineering Conference, ASME BED-Vol. 35: 189-190.

Yeni, Y.N., Brown, C.U. and Norman, T.L., 1996, The influence of biochemical and morphological constituents of human bone on microdamage. Advances in Bioengineering, ASME 
BED-Vol. 33: 309-310.

Brown, C., Norman, T.L. and Wang, Z., 1996, Microdamage influences fracture toughness of human bone. Transactions of the 42nd Annual Meeting, Orthopaedic Research Society, Atlanta, GA, February 19-22, p. 58.

Brown, C. and Norman, T.L., 1995, Fracture toughness of human cortical bone from the proximal femur. Advances in Bioengineering, ASME BED-Vol. 31: 121-122.

Brown, C., 1994, Surgical cement, stainless steel stem interface strength determined by push out tests. Department of Mechanical and Aerospace Engineering and Orthopedics, West Virginia University, April.

\section{PRESENTATIONS}

Time-dependent circumferential deformation of bone specimens subjected to internal radial loading. $22^{\text {nd }}$ SAMPE Europe International Conference, CNIT Conference Centre, La Defense, Paris, France, March 26, 2001, oral presentation.

Time-dependent circumferential deformation of bone specimens subjected to internal radial loading. Fifth Annual Research Review Presentation, Department of Orthopedics, West Virginia University, Morgantown, WV, March 16, 2001, oral presentation.

Circumferential creep of human cortical bone suggests threshold for press-fit stems. 47th Annual Meeting, Orthopaedic Research Society, San Francisco, CA, February 26, 2001, oral presentation.

Mechanisms of creep induced failure in cylindrical cortical bone specimens due to radial loading. ASME International Mechanical Engineering Congress and Exposition, Bioengineering Division Winter Annual Meeting, November 10, 2000, Orlando, Florida, oral presentation.

An introduction to proteins: structure and function. Musculoskeletal Research Center, Department of Orthopedics, West Virginia University, June 30, 2000, oral presentation.

Time-dependent circumferential deformation of bone specimens subjected to internal radial loading. Fourth Annual Research Review Presentation, Department of Orthopedics, West Virginia University, Morgantown, WV, June 2, 2000, oral presentation.

Time-dependent circumferential deformation of bone specimens subjected to internal radial loading. $45^{\text {th }}$ International SAMPE Symposium and Exhibition, Long Beach, CA, May 21-25, 2000 , oral presentation.

Circumferential strain around femoral cylinders due to simulated press-fit loading. BaltimoreWashington, D.C. SAMPE Chapter 2000 Student Symposium, University of Maryland, College Park, February 9, 2000, poster presentation.

Transverse creep response in human femoral bone. $23^{\text {rd }}$ Annual Meeting, American Society of Biomechanics, October 21-23, 1999, Pittsburgh, PA, oral presentation.

Transverse creep behavior of cortical bone. Third Annual Research Review Presentation, Department of Orthopedics, West Virginia University, Morgantown, WV, May 21, 1999, oral presentation. 
Circumferential strain around femoral cylinders due to simulated press-fit loading. West Virginia University School of Medicine Research Day, March 19, 1999, poster presentation.

Fracture toughness of the femoral neck, femoral shaft and tibial shaft in aged bone. ASME International Mechanical Engineering Congress and Exposition, Bioengineering Division Winter Annual Meeting, November 15-20, 1998, Anaheim, California, oral presentation.

Circumferential strain around femoral cylinders due to simulated press-fit loading. ASME International Mechanical Engineering Congress and Exposition, Bioengineering Division Winter Annual Meeting, November 15-20, 1998, Anaheim, California, poster presentation.

The microstructure of human bone influences fracture toughness similar to fiber-reinforced composites. 43rd International SAMPE Symposium and Exhibition, May 31 to June 4, 1998, Anaheim, California. Ph.D. student paper competition, oral presentation.

Circumferential strain around femoral cylinders due to simulated press-fit. Second Annual Research Review Presentation, Department of Orthopedics, West Virginia University, Morgantown, WV, May 29, 1998, oral presentation.

The influence of bone microstructure and composition on the fracture toughness of human cortical bone. Baltimore-Washington, D.C. SAMPE Chapter 1998 Student Symposium, University of Maryland, College Park, February 11, 1998, poster presentation

Transverse creep response of femoral bone and its applications. First Annual Research Review Presentation, Department of Orthopedics, West Virginia University, Morgantown, WV, April 24, 1997, oral presentation.

Fracture toughness of human cortical bone from the proximal femur. International Mechanical Engineering Congress and Exposition, November 12-17, 1995, San Francisco, California. Masters student paper competition oral presentation.

Conceptual design of an orbital debris defense system. 10 th Annual Summer Conference, NASA/USRA Advanced Space Design Program, Pasadena, CA, June 13-16, 1994.

Surgical cement, stainless steel stem interface strength determined by push out tests. Presented to Department of Mechanical and Aerospace Engineering and the Department of Orthopedics, West Virginia University, Spring, 1994.

\section{RELEVANT CLASSES}

- Biomedical: Cellular and Molecular Biochemistry (2 semesters), Gross Anatomy, Physiology (Mechanisms of Body Functions), Bioengineering, Bioengineering Seminar

- Solid Mechanics and Materials: Instrumentation in Engineering, Advanced Mechanics of Materials, Experimental Stress Analysis, Fracture Mechanics, Energy Methods Applied Mechanics, Theory of Elasticity, Mechanics of Metallurgy, Theory of Plasticity, Continuum Mechanics, Microscopy (SEM and Optical), Introduction to Finite Elements

- Science: General Physics (2 semesters), General Chemistry (2 semesters)

- Mathematics: Calculus, Differential Equations, Numerical Analysis, Applied Linear Algebra, Applied Regression Analysis, Advanced Calculus 


\section{REFERENCES}

Dr. Timothy L. Norman, Professor

Director of the Musculoskeletal Research Center, Department of Orthopedics

Department of Mechanical and Aerospace Engineering/

Department of Orthopedics, PO Box 9196, Health Sciences Center South, West Virginia University

Morgantown, WV 26506-9196

Telephone: (304) 293-1072

email: tnorman@wvu.edu

Dr. J. David Blaha, Professor

Chairman, Department of Orthopedics

Department of Orthopedics, PO Box 9196, Health Sciences Center South, West Virginia University

Morgantown, WV 26506-9196

Telephone: (304) 293-7393

email:jblaha@wvu.edu

Tom Gruen, Adjunct Associate Professor

Department of Orthopedics, PO Box 9196, Health Sciences Center South, West Virginia University

Morgantown, WV 26506-9196

Telephone: (304) 293-1177

email: tgruen@wvu.edu

Vincent L. Kish, Laboratory Instrumentation Specialist

Department of Orthopedics, PO Box 9196, Health Sciences Center South, West Virginia University

Morgantown, WV 26506-9196

Telephone: (304) 293-1071

email: vkish@wvu.edu

Nina Clovis, Research Assistant

Department of Orthopedics, PO Box 9196, Health Sciences Center South, West Virginia University

Morgantown, WV 26506-9196

Telephone: (304) 293-3624

email:nclovis@wvu.edu 